Solução numérica de equações diferenciais parciais implícitas de primeira ordem 

SERVIÇO DE PÓS-GRADUAÇ̃̃O DO ICMC-USP

Data de Depósito:11 de dezembro de 2014

Assinatura:

\title{
Solução numérica de equações diferenciais parciais implícitas de primeira ordem
}

\author{
Sergio Moisés Aquise Escobedo
}

Orientador: Prof. Dr. Antonio Castelo Filho

Coorientador: Prof. Dr. Marcio Fuzeto Gameiro

Dissertação apresentada ao Instituto de Ciências Matemáticas e de Computação - ICMC-USP, como parte dos requisitos para obtenção do título de Mestre em Ciências - Ciências de Computação e Matemática Computacional. VERSÃO REVISADA 
Ficha catalográfica elaborada pela Biblioteca Prof. Achille Bassi e Seção Técnica de Informática, ICMC/USP, com os dados fornecidos pelo(a) autor(a)

\begin{tabular}{|c|c|}
\hline \multirow[t]{3}{*}{ EE74ss } & $\begin{array}{l}\text { Escobedo, Sergio Moisés Aquise } \\
\text { Solução numérica de equações diferenciais parciais } \\
\text { implícitas de primeira ordem / Sergio Moisés Aquise } \\
\text { Escobedo; orientador Antonio Castelo Filho; co- } \\
\text { orientador Márcio Fuzeto Gameiro. -- São Carlos, } \\
2014 . \\
\quad 110 \text { p. }\end{array}$ \\
\hline & $\begin{array}{l}\text { Dissertação (Mestrado - Programa de Pós-Graduação } \\
\text { em Ciências de Computação e Matemática } \\
\text { Computacional) -- Instituto de Ciências Matemáticas } \\
\text { e de Computação, Universidade de São Paulo, } 2014 .\end{array}$ \\
\hline & $\begin{array}{l}\text { 1. Equações diferenciais parciais. 2. Métodos } \\
\text { numéricos. 3. Métodos de continuação numérica. I. } \\
\text { Castelo Filho, Antonio, orient. II. Gameiro, Márcio } \\
\text { Fuzeto, co-orient. III. Título. }\end{array}$ \\
\hline
\end{tabular}


Probamos por medio de la lógica, pero descubrimos por medio de la intuición.

Henri Poincaré 

A minha Esposa e minhas Filhas. 
A minha Mãe e meus Irmãos. 


\section{Agradecimentos}

A Deus pela vida, saúde e apoio incondicional.

A CAPES pelo apoio financeiro para o desenvolvimento deste trabalho.

Ao Instituto de Ciências Matemáticas e de Computação da Universidade de São Paulo e à Universidad Nacional de San Agustín de Arequipa pela oportunidade de fazer meus estudos de Mestrado.

Ao Dr. Valdemar Medina Hoyos e ao Dr. Jorge Luis Salas Arenas quem fizeram possível minha permissão de estudos.

Ao Dr. German Chavez Contreras reitor da Universidad Catótica San Pablo de Arequipa pelo apoio concedido.

Ao professor Antonio Castelo Filho, pela orientação, dedicação e vontade na realização deste trabalho e por todos os conhecimentos transmitidos.

Ao professor Márcio Fuzeto Gameiro pela coorientação e disposição para o desenvolvimento deste trabalho.

Aos professores do ICMC da Universidade de São Paulo, em especial ao professor Victor Hugo Jorge Perez pelo apoio e incentivo.

Aos membros da banca julgadora, pelas sugestões e correções.

A minha esposa Claudia e a minhas Filhas Jasmine, Natalia, Claudia e Valeria por seu esforço, coragem e amor ao ficar distanciados nestos dois anos.

A todos meus amigos em especial a : Edwin Choquehuanca, Stevens Paz, Daniel Gutierrez, Rafael Almeida, Nadia Borge, Regina Medeiros e Freddy Begazo, por estar presentes em diversas situações de minha vida. 


$S$ equações diferencias parciais tem origem na modelagem do problemas nas ciências e engenharia, tais como a equação do calor, equação da onda, equação de Poisson, entre outras. Para muitas destas equações não é tão simples obter uma técnica analítica para achar sua solução e nestes casos é necessário uso de soluções aproximadas obtidas pelo computador. Existem técnicas tradicionais para solução numérica de uma grande classe de equações diferenciais, mas quando esta equação está na forma implícita, muitas destas técnicas já não podem ser aplicadas.

Frequentemente as equações diferenciais parciais de segunda ordem tem maior estudo que as equações de primeira ordem sendo uma das razões que os modelos envolvem derivadas de segunda ordem. No caso das equações diferenciais parciais de primeira ordem implícitas a não linearidade em alguns casos não permite determinar uma solução de forma simples.

O trabalho desenvolvido faz uma revisão do método das características para estabelecer as condições necessárias e suficientes, que permitam encontrar uma solução, ao mesmo tempo evidencia a complexidade de determinar uma solução clássica. Dentro das aplicações existentes relacionadas com as Equações Diferenciais Parciais Implícitas de Primeira Ordem, podemos mencionar a Equação cinemática e a Equação de Hamilton-Jacobi que podem-se associar com o movimento de partículas. Para a solução de uma Equação Diferencial Implícita de Primeira Ordem o método das características tem uma estrutura de solução que permite resolver a equação de forma analítica e numérica, desde que se verifique o Teorema de Cauchy.

O objetivo deste trabalho de mestrado é obter um método numérico para a solução de equações diferenciais parciais de primeira ordem implícitas. Nós propomos um método numérico do tipo previsor-corretor que resolve uma EDP de primeira ordem implícita, utilizando o sistema característico em conjunto com as condições de banda, para reduzir o erro global nas iterações. 

$\mathrm{P}$ Artial differential equations arise in the modeling of problems in science and engineering, such as the heat equation, wave equation, Poisson equation, among others. For many of these equations it is not so simple to obtain an analytical technique to find a solution in these cases and it is necessary to use a computer to obtain approximate solutions. There are traditional techniques for numerical solution of a large class of differential equations, but when this equation is in implicit form, many of these techniques can no longer be applied.

Often partial differential equations of second order are more studied than first order equations the reason being that one of the models involve secondorder derivatives. In the case of implicit partial differential equations of first order the non-linearity in some cases does not allow for a solution in simple from to be determined.

The work reviews the method of characteristics to establish the necessary and sufficient conditions that will find a solution at the same time demonstrates the complexity of determining classical solution. Within existing applications related to Partial Differential Equations of First Order Implicit, we can mention the textit kinematic equation and textit equation Hamilton-Jacobi that can be associated with the movement of particles. For the solution of a differential equation First Implicit Order the method of characteristics has a solution framework that enables solve the equation analytically and numerically, provided there is the Cauchy theorem.

The objective of this master thesis is to obtain a numerical method for the solution of partial differential equations first order implicit. We propose a numerical method of predictor-corrector type that resolves a EDP first implicate order, using the characteristic system in conjunction with the band conditions, to reduce the overall error in iterations. 



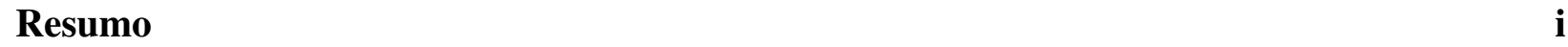

$\begin{array}{lll}\text { Abstract } & \text { iii }\end{array}$

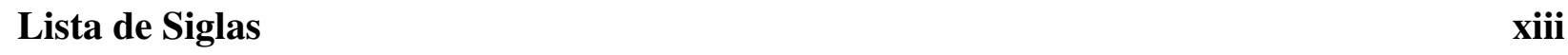

1 Conceitos básicos $\quad 1$

1.1 Equação Diferencial Parcial Implícita de Primeira Ordem . . . . . . . . . . . . . . 3

1.2 Classificação das Equações Diferenciais Parciais de Primeira Ordem . . . . . . . . . 4

1.3 Solução de uma Equação Diferencial Parcial de Primeira Ordem . . . . . . . . . . 6

1.4 Teoria sobre as curvas características . . . . . . . . . . . . . . . 7

1.5 Solução de EDPs Lineares usando o método das características . . . . . . . . . . . 8

1.6 Solução de EDPs Não Lineares pelo método das características . . . . . . . . . . . . 22

2 Existência e unicidade da solução $\quad 33$

2.1 Teorema De Cauchy . . . . . . . . . . . . . . . . . . . . . 34

2.2 Teorema de Cauchy generalizado . . . . . . . . . . . . 35

3 Solução Numérica $\quad 39$

3.1 Solução Numérica de Equações Diferenciais Ordinárias . . . . . . . . . . . . . . . . . 40

3.2 Passo adaptativo Método de Runge-Kutta-Fehlberg . . . . . . . . . . . . . . . . . 43

3.3 Métodos Lineares Multi-passo . . . . . . . . . . . . . . . . . . . . . 45

3.4 Método Previsor-Corretor de Adams-Bashforth- Moulton . . . . . . . . . . . . . . 46

3.5 Método Previsor-corretor de Euler-Newton . . . . . . . . . . . . . . . 47

4 Resultados 49

4.1 Curvas características de EDPs de primeira ordem . . . . . . . . . . . . . 58

4.2 Solução numérica de EDPs lineares e quase-lineares de primeira ordem . . . . . . 60

4.3 Solução numérica de EDPs implícitas de primeira ordem . . . . . . . . . . . . . . . 63

4.4 Equação de Burgers . . . . . . . . . . . . . . . . . . . . . . . . . . . . . . . . . . . . . . . . . . . . .

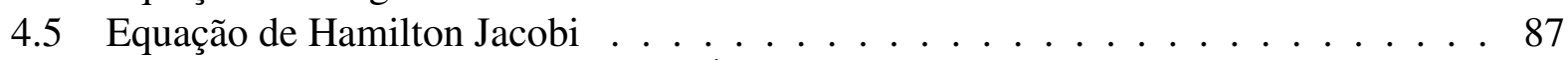

4.6 Algumas aplicações na Mecânica e Ótica . . . . . . . . . . . . . . . . . . 90

5 Conclusões da Dissertação $\quad 95$

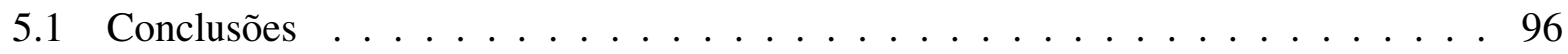

5.2 Trabalhos futuros . . . . . . . . . . . . . . . . . 97 
A Solução de equações diferenciais sobre variedades 103

B Métodos numéricos para equações algébrico diferenciais $\quad 107$

C Equivalência de Equações Diferenciais Parciais de Primeira Ordem e Problemas Variacionais 


\section{Lista de Figuras}

1.1 PL Approximation to Manifolds and Its Application to Implicit ODEs:Imagens retiradas de Lectures in Applied Mathematics volume 26 Computational Solution of Nonlinear Systems of Equations (Castelo e Tavares, 1990) . . . . . . . . . . . . 2

1.2 Método da envoltória e curvas características . . . . . . . . . . . . . . . 3

1.3 Curvas características . . . . . . . . . . . . . . . . . 7

1.4 Curva característica parametrizada . . . . . . . . . . . . . . . . 8

1.5 Relação da Curva característica com o plano tangente . . . . . . . . . . . . . . . 9

1.6 Relação entre a solução de uma EDP e seus curvas características . . . . . . . . . . . 10

1.7 Solução paramétrica . . . . . . . . . . . . . . . . . . . . . 11

1.8 Curva caraterística gerada pela curva característica inicial . . . . . . . . . . . . 12

1.9 Solução $u(x, y)=f(x-0.5 y) \quad \ldots \ldots \ldots \ldots \ldots \ldots$

1.10 Ondas viajantes parabólicas . . . . . . . . . . . . . . . . . . 14

1.11 Ondas viajantes delta Dirac . . . . . . . . . . . . . . . . . . . . . 14

1.12 Ondas viajantes descontinuas . . . . . . . . . . . . . . . . . . . . . . . . . . . . . . . . . . . . .

1.13 Ondas viajantes não diferenciáveis . . . . . . . . . . . . . . . . 15

1.14 Solução da equação $x u_{x}+y u_{y}=c u$ com condição inicial $u(x, 1)=e^{-x^{2}}$ para $c=-1 \ldots \ldots \ldots \ldots \ldots \ldots \ldots$

1.15 Curvas características e singularidade . . . . . . . . . . . . . . . 17

1.16 Singularidade apresentada na solução da equação $x u_{x}+y u_{y}=c u$ com condição inicial $u(x, 1)=e^{-x^{2}}$ para $c=-1 \ldots \ldots \ldots \ldots \ldots$

1.17 Singularidade e Curvas características . . . . . . . . . . . . . . 18

1.18 Curvas características da equação $u_{x}+y u_{y}=0$, com condição inicial $u(0, y)=$

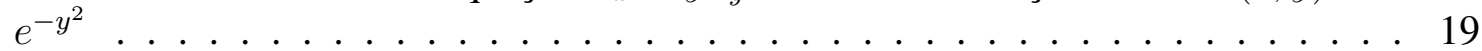

1.19 Solução da equação $u_{x}+y u_{y}=0$, com condição inicial $u(0, y)=e^{-y^{2}} \ldots \ldots$

1.20 Solução da equação $2 u_{x}+u_{y}=c u, \operatorname{com} c=0$ e condição inicial $u(x, 0)=\cos x \quad$. 20

1.21 Solução da equação $2 u_{x}+u_{y}=c u, \operatorname{com} c=1$ e condição inicial $u(x, 0)=\cos x \quad$. 21

1.22 Solução da equação $e^{y} u_{x}+u_{y}=c u, \operatorname{com} c=0$ e condição inicial $u(x, 0)=\cos x \quad 21$

1.23 Solução da equação $e^{y} u_{x}+u_{y}=c u, \operatorname{com} c=1$ e condição inicial $u(x, 0)=\cos x \quad 22$

1.24 Solução da equação $\quad u u_{x}+u_{y}=0$ com condição inicial $u(x, 0)=-x \ldots$. . . 23

1.25 Solução da equação $u u_{x}+u_{y}=0$ com condição inicial $u(x, 0)=x \ldots \ldots$. . . . 24

1.26 Visualização da solução $u(x, y)=e^{-(x-0.5 y)^{2}}$ usando Marching tetrahedra . . . . . 28

1.27 Visualização da solução $x^{2}+y^{2}=(1-u)^{2}$ usando Marching tetrahedra . . . . . . 30

1.28 Visualização da solução $u(x, y)=e^{2 y}+(x-1) e^{-y}$ usando Marching tetrahedra . 32

3.1 Aproximações da derivada . . . . . . . . . . . . . . . . . 41 
3.2 Passo constante e Erro de truncamento . . . . . . . . . . . . . . . . . . . 42

3.3 Passo adaptativo . . . . . . . . . . . . . . . . . . . . . . 43

3.4 Método Previsor- Corretor . . . . . . . . . . . . . . . . . . 47

4.1 Superfície paramétrica $\ldots \ldots \ldots \ldots \ldots$. . . . . . . . . . . 50

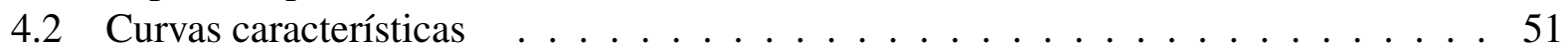

4.3 Soluções usando sistemas de ODEs . . . . . . . . . . . . . . . . . . . 52

4.4 Curvas Características aproximadas pelo sistema Característico . . . . . . . . . 52

4.5 Curva solução inicial parametrizada e Curva característica parametrizada . . . . . . 54

4.6 Geração da Superfície usando uma curva característica (solução inicial) . . . . . . 55

4.7 Interface no Matlab para o Menu de opções . . . . . . . . . . . . . . . . . . 57

4.8 Curvas características da equação $a u_{x}+u_{y}=0 \ldots \ldots \ldots \ldots$

4.9 Superfície solução da EDP $x u_{x}+y u=c u, c=-1$ e $u(x, 1)=e^{-x^{2}} \ldots \ldots$. . . . 58

4.10 Curvas características da EDP $x u_{x}+y u=c u, c=-1$ e $u(x, 1)=e^{-x^{2}} \ldots \ldots$

4.11 Superfície solução da EDP $x u_{x}+y u=c u, c=1$ e $u(x, 1)=\exp \left(-x^{2}\right) \ldots \ldots$. . 59

4.12 Curvas características da EDP $x u_{x}+y u=c u, c=1 \mathrm{e} u(x, 1)=e^{-x^{2}} \ldots \ldots$

4.13 Solução Numérica da equação $-y u_{x}+x u_{y}=4 x y, \operatorname{com} u(x, 0)=\cos (x)$, usando Runge-Kutta de quinta ordem . . . . . . . . . . . . . . . . . . 6 61

4.14 Solução Numérica da equação $e^{y} u_{x}+u_{y}=u$, com $u(x, 0)=\cos (x)$, usando Runge-Kutta de quinta ordem . . . . . . . . . . . . . . . . . . 6 61

4.15 Solução analítica da equação $e^{y} u_{x}+u_{y}=u \ldots \ldots$. . . . . . . . . . 62

4.16 Solução Numérica de $u u_{x}+u u_{y}=-x-y$, com $u(x, 0)=\sqrt{1-x^{2}}$, usando Runge-Kutta de quinta ordem . . . . . . . . . . . . . . . . . 62

4.17 Solução numérica bilateral de $u u_{x}+u u_{y}=-x-y$, com $u(x, 0)=\sqrt{1-x^{2}}$, usando Runge-Kutta de quinta ordem . . . . . . . . . . . . . . . . . 62

4.18 Solução numérica da equação $\left(u_{x_{1}}\right)^{2}+\left(u_{x_{2}}\right)^{2}=1$, com condição inicial $x_{1}{ }^{2}+$ $x_{2}{ }^{2}=1, u=0$, usando o Método de Euler . . . . . . . . . . . . . . . 64

4.19 Solução numérica da equação $u u_{x_{1}}+u_{x_{2}}=0$, com condição inicial $u\left(x_{1}, 0\right)=$ $-x_{1}$, usando o Método de Euler . . . . . . . . . . . . . . . . . 64

4.20 Solução numérica da EDP $x_{1}+x_{2}+u p_{1}+u p_{2}=0$, com $x_{1}{ }^{2}+u^{2}=1$, usando o Método de Euler . . . . . . . . . . . . . . . . . . . . . .

4.21 Solução numérica da EDP $x_{1}+x_{2}+u p_{1}+u p_{2}=0$, com $x_{1}^{2}+u^{2}=1$, usando o Método de Euler

4.22 Solução numérica da equação $\left(u_{x_{1}}\right)^{2}+\left(u_{x_{2}}\right)^{2}=1$, com condição inicial $x_{1}^{2}+$ $x_{2}{ }^{2}=1, u=0$, usando o Método de Runge-Kutta de ordem 5

4.23 Solução numérica da EDP $x_{1}+x_{2}+u p_{1}+u p_{2}=0$, com $x_{1}{ }^{2}+u^{2}=1$, usando o Método de Runge-Kutta de ordem 5

4.24 Solução numérica da EDP $x_{1}+x_{2}+u p_{1}+u p_{2}=0$, com $x_{1}^{2}+u^{2}=1$, usando o Método de Runge-Kutta de ordem $5 \ldots \ldots \ldots$. . . . . . . . . . .

4.25 Solução numérica da EDP $x_{2} p_{1}+x_{1} p_{2}-4 x_{1} x_{2}=0$, com $u\left(x_{1}, 0\right)=\cos \left(x_{1}\right)$, usando o Método de Runge-Kutta de ordem $5 \ldots$. . . . . . . . . . . . . 67

4.26 Curvas características com passo constante . . . . . . . . . . . . . . . . 68

4.27 Solução numérica da equação $\left(u_{x_{1}}\right)^{2}+\left(u_{x_{2}}\right)^{2}=1$, com $x_{1}{ }^{2}+x_{2}{ }^{2}=1, u=0$, usando o Método de Adams-Bashforth-Moulton . . . . . . . . . . . . . . . . . 68

4.28 Solução numérica da EDP $x_{1}+x_{2}+u p_{1}+u p_{2}=0$, com $x_{1}{ }^{2}+u^{2}=1$, usando o Método de Adams-Bashforth-Moulton . . . . . . . . . . . . . . . . .

4.29 Solução numérica da equação $F\left(x_{1}, x_{2}, u, p_{1}, p_{2}\right)=p_{1}{ }^{2}+p_{2}+u=0$, com condição inicial $u\left(x_{1}, 0\right)=x_{1}$, usando o Método de Adams-Bashforth-Moulton 
4.30 Solução numérica da equação $F\left(x_{1}, x_{2}, u, p_{1}, p_{2}\right)=x_{1}+x_{2}+u p_{1}+u p_{2}=0$, com condição inicial $x_{1}^{2}+u^{2}=1$, usando o Método de Euler . . . . . . . . . . . .

4.31 Solução numérica da equação $F\left(x_{1}, x_{2}, u, p_{1}, p_{2}\right)=p_{1}{ }^{2}+p_{2}+u=0$, com condição inicial $u\left(x_{1}, 0\right)=x_{1}$, usando o Método de Euler . . . . . . . . . . . . . . .

4.32 Solução numérica da equação $F\left(x_{1}, x_{2}, u, p_{1}, p_{2}\right)=x_{1}+x_{2}+u p_{1}+u p_{2}=0$, com condição inicial $x_{1}^{2}+u^{2}=1$, para $\alpha(t)=(\cos (t), 0, \sin (t)), t \in[0,3]$, usando o Método de Runge-Kutta de ordem 2

4.33 Solução numérica da equação $F\left(x_{1}, x_{2}, u, p_{1}, p_{2}\right)=x_{1}+x_{2}+u p_{1}+u p_{2}=0$, com condição inicial $x_{1}^{2}+u^{2}=1$, para $\alpha(t)=(\cos (t), 0, \sin (t)), t \in[0,6]$, usando o Método de Runge-Kutta de ordem $2 \ldots \ldots \ldots$. . . . . . . . . . .

4.34 Solução numérica da equação $\left(u_{x_{1}}\right)^{2}+\left(u_{x_{2}}\right)^{2}=1$, com $x_{1}^{2}+x_{2}^{2}=1, u=0$, usando o Método tipo Projeção de Euler-Newton

Solução numérica da equação $u_{x_{1}} u_{x_{2}}=u$, com $u\left(0, x_{2}\right)=x_{2}^{2}$, usando o Método tipo Projeção de Euler-Newton . . . . . . . . . . . . . . . . . . . . .

4.36 Solução numérica da equação $u_{x}+u_{y}=0$, com $u(x, 0)=1-x$, usando o método de Runge-Kutta de ordem 5 . . . . . . . . . . . . . . . . . . . . .

4.37 Curvas características e solução analítica da equação $u_{x}+u_{y}=0, \operatorname{com} u(x, 0)=$

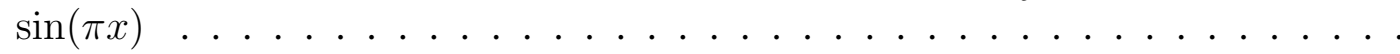

4.38 Comparação da Solução analítica e as curvas características da equação $u_{x}+u_{y}=$ $0, \operatorname{com} u(x, 0)=\sin (\pi x) \ldots \ldots \ldots \ldots \ldots$

4.39 Solução numérica da equação $u_{y}+u^{2} u_{x}=0$, com $u(x, 0)=1-x$, usando o Método de Runge-Kutta de ordem $5 \ldots \ldots \ldots \ldots$

4.40 Solução numérica da equação $u_{y}+u^{2} u_{x}=0$, com $u(x, 0)=1-x$, usando o Método de Runge-Kutta de ordem $5 \ldots \ldots$. . . . . . . . . . . . .

4.41 Solução numérica da equação $u_{y}+u u_{x}=0$, com $u(x, 0)=1-x$, usando Runge-

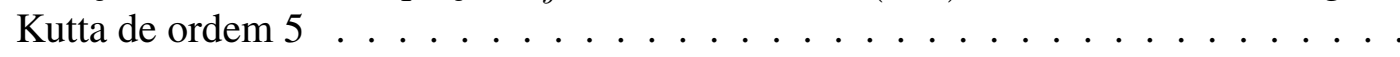

4.42 Solução numérica da equação $u_{y}+u u_{x}=0$, com $u(x, 0)=1-x$, usando RungeKutta de ordem 5

4.43 Parametrização da solução numérica para a equação $u_{y}+u u_{x}=0, \operatorname{com} u(x, 0)=$ $1-x$, usando o Método de Runge-Kutta de ordem $5 \ldots$. . . . . . . . . .

4.44 Solução analítica e Solução numérica da equação $u_{y}+u u_{x}=0$, sendo $u(x, 0)=$ $\sin \left(x^{2}\right)$, usando o Método de Runge-Kutta de ordem $5 \ldots$. . . . . . . . . . .

4.45 Comparação da Solução analítica com as curvas características da equação $u_{y}+$ $u u_{x}=0$, sendo $u(x, 0)=\sin \left(x^{2}\right) \ldots \ldots \ldots \ldots$. . . . . . . . . . . . . 83

4.46 Solução numérica da equação $u_{y}+u u_{x}=0, \operatorname{com} u(x, 0)=\sin (\pi x) \ldots$. . . 83

4.47 Solução numérica da equação $u_{y}+u u_{x}=0, \operatorname{com} u(x, 0)=\sin (\pi x) \ldots \ldots$. . . 84

4.48 Parametrização da solução . . . . . . . . . . . . . . . . . . . . . . . . . 84

4.49 Comparação das curvas características e a solução analítica da equação $u_{y}+u u_{x}=$ $0, \operatorname{com} u(x, 0)=\sin (\pi x) \ldots \ldots \ldots \ldots \ldots$

4.50 Vista superior das curvas características . . . . . . . . . . . . . . 85

4.51 Onda viajante da equação $u_{y}+u u_{x}=0, \operatorname{com} u(x, 0)=e^{-x^{2}} \ldots \ldots$. . . . . 85

4.52 Curvas características e choques da equação $u_{y}+u u_{x}=0$, com $u(x, 0)=e^{-x^{2}}$. 86

4.53 Formação de choques . . . . . . . . . . . . . . . . . . 86

4.54 Formação de choques $u_{y}+u u_{x}=0$, sendo $u(x, 0)=e^{-x^{2}} \ldots \ldots$. . . . . . 86

4.55 Frentes de onda e curvas características . . . . . . . . . . . . . . . . 90

4.56 Frentes de onda e curvas características correspondentes à equação $\left(u_{x}\right)^{2}+\left(u_{y}\right)^{2}=$ $1, \operatorname{com} x^{2}+y^{2}=1, u=0 \ldots \ldots \ldots \ldots \ldots$

4.57 Geodésica : Figura retirada de Classical Mechanics, R. Gouglas Gregory . . . . . . 91 
4.58 Ação funcional e frentes de onda . . . . . . . . . . . . . . . . . . . . . 91

4.59 Energia Potencial e espaço de fase : Figura retirada de Mathematical Methods of Classical Mechanics, V. I. Arnold _. . . . . . . . . . . . . . . . . 93

4.60 Curvas fase e campo de vetores gradiente da função $f(x, y)=x e^{-x^{2}-y^{2}} \ldots$. . . 93

4.61 Solução da equação $u_{t}+\frac{1}{2} u_{x}^{2}=0, \operatorname{com} u(x, 0)=-x^{2} \ldots \ldots \ldots$. . . . . . 94

A.1 Esfera . . . . . . . . . . . . . . . . . . . 103

A.2 Vetores tangente de curvas sobre uma esfera . . . . . . . . . . . . . . . 104

A.3 Espaço Tangente e Método previsor corretor . . . . . . . . . . . . . . . . . . . 104

A.4 Solução das Equações do movimento sobre uma esfera . . . . . . . . . . . . . . . 105 


\section{Lista de Tabelas}

4.1 Tabela de comparação do erro pelo número de iterações, ao resolver a equação $\left(u_{x_{1}}\right)^{2}+\left(u_{x_{2}}\right)^{2}=1$, com $x_{1}^{2}+x_{2}^{2}=1, u=0$, Método de projeção de EulerNewton . . . . . . . . . . . . . . . . . . 72

4.2 Tabela de comparação do erro pelo número de iterações, ao resolver a equação $\left(u_{x_{1}}\right)^{2}+\left(u_{x_{2}}\right)^{2}=1, \operatorname{com} x_{1}{ }^{2}+x_{2}{ }^{2}=1, u=0$, Método de projeção R.K.4-Newton 72

4.3 Erro cometido ao resolver a equação $\left(u_{x_{1}}\right)^{2}+\left(u_{x_{2}}\right)^{2}=1, \operatorname{com} x_{1}{ }^{2}+x_{2}{ }^{2}=1, u=0 \quad 73$

4.4 Erro cometido ao resolver a equação $\left(u_{x_{1}}\right)^{2}+\left(u_{x_{2}}\right)^{2}=1, \operatorname{com} x_{1}{ }^{2}+x_{2}{ }^{2}=1, u=0 \quad 73$

4.5 Tabela de comparação do erro pelo número de iterações, ao resolver a equação $u_{x_{1}} u_{x_{2}}=u, \operatorname{com} u\left(0, x_{2}\right)=x_{2}^{2}$, Método de projeção de Euler-Newton $\ldots$. . . . 74

4.6 Tabela de comparação do erro pelo número de iterações, ao resolver a equação $u_{x_{1}} u_{x_{2}}=u$, com $u\left(0, x_{2}\right)=x_{2}^{2}$, Método de projeção R.K.4-Newton $\ldots . .74$

4.7 Erro cometido ao resolver a equação $u_{x_{1}} u_{x_{2}}=u, \operatorname{com} u\left(0, x_{2}\right)=x_{2}{ }^{2} \ldots \ldots$. . 75

4.8 Erro cometido ao resolver a equação $u_{x_{1}} u_{x_{2}}=u$, com $u\left(0, x_{2}\right)=x_{2}{ }^{2} \ldots \ldots$ 



\section{Lista de Siglas}

EDP - Equação Diferencial Parcial

EDO - Equação Diferencial Ordinaria

EAD - Equação Algébrico Diferencial

EDPs - Equações Diferenciais Parciais

EDOs - Equações Diferenciais Ordinarias

EADs - Equações Algébrico Diferenciais

PVI - Problema de Valor Inicial 


\section{Conceitos básicos}

Em geral, referenciar uma equação diferencial parcial faz imaginar algum modelo físico que seja descrito por esta equação e ao mesmo tempo pensa-se em procurar algum método que possa determinar sua solução. Ao longo do tempo muitas pessoas resolveram casos particulares com o objetivo de estabelecer soluções analíticas e numéricas sujeitas a diferentes condições. Além disso, diversos estudos na área concluem que pensar numa solução geral para todos os casos ainda não é factível, mas para um caso específico pode-se obter uma "solução geral" .

Habitualmente se prova a existência de uma solução para depois obter métodos de solução analíticos ou numéricos. Podemos mencionar alguns dos métodos analíticos mais conhecidos que resolvem equações diferenciais parciais de primeira ordem (Faro, 2013), tais como, o método das características de Cauchy, método da projeção e método da envoltória (Figura 1.2(a)).

Com respeito aos Métodos Numéricos que resolvem equações diferenciais, podemos mencionar que as equações diferenciais parciais de segunda ordem, são as mais estudadas pela maior importância devido a seus diversas aplicações na Física. Foi Lagrange quem estudou os aspectos teóricos acerca das equações diferenciais parciais de primeira ordem na forma $F\left(x, y, u, u_{x}, u_{y}\right)=0$, entre os anos 1772 e 1779. Ideias geométricas foram desenvolvidas por Gaspar Monge entre 1770 e 1784 associando a cada equação, um cone em cada ponto do espaço sendo as soluções superfícies tangentes a estes cones.

Desde o século $X I X$ com o uso dos computadores, tem sido feitos trabalhos com bastante rigor no cálculo computacional para determinar soluções numéricas e representações gráficas (figura 1.1). Citando alguns trabalhos temos: 


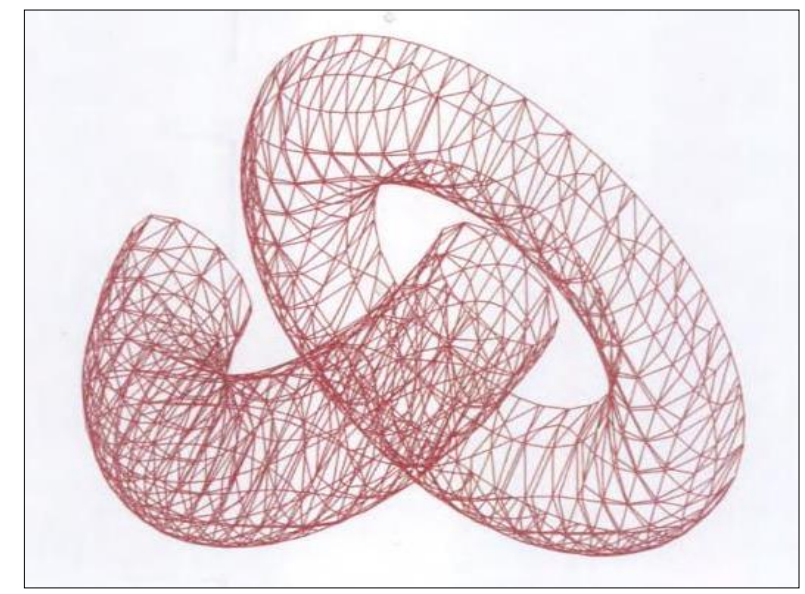

Figura 1.1: PL Approximation to Manifolds and Its Application to Implicit ODEs:Imagens retiradas de Lectures in Applied Mathematics volume 26 Computational Solution of Nonlinear Systems of Equations (Castelo e Tavares, 1990)

A. Castelo \& S. De Freitas \& G.Tavares (Castelo e Tavares, 1990), descrevem um algoritmo resolver uma equação diferencial implícita da forma $F\left(x, y, y^{\prime}\right)=0$, utilizando técnicas de desingularização associando uma superfície à equação.

E.Hairer (Hairer, 2000), descreve um método numérico para resolver equações diferenciais sobre variedades denominado Método da projeção simétrica.

M.C. Bertin: Pimentel, B.M. ; and Pompeia, P.J (Bertin e Pompeia, 2007), em seu trabalho, descrevem a analogia que existe entre a teoria das Equações Diferenciais Ordinárias EDOs a teoria das equações diferenciais parciais EDPs e o cálculo variacional mediante a equação de HamiltonJacobi esta analogia permite estabelecer uma interpretação geométrica.

A. Castelo \& G.Tavares (Castelo e Tavares, 2010) descrevem num relatório técnico um método para resolver equações diferenciais implícitas com singularidades,baseado em métodos de continuação, para equações da forma $F\left(X, Y, Y^{\prime}(X), \ldots, Y^{(k)}(X)\right)=0$.

A importância da solução das EDPs se encontram na modelação de fenômenos da ciência e tecnologia que descrevem sua evolução ao longo do tempo, dai que é preciso determinar sua solução, por exemplo a equação do transporte $u_{t}+u_{x}=0$ é um modelo unidimensional que permite compreender equações da mecânica dos fluidos multidimensional como as equações de Euler e Navier-Stokes, a equação do transporte linear tem importância por que algumas equações de ordem dois que modelam fenômenos como propagação de ondas recaem sobre a equação do transporte linear.

Para garantir a existência de uma solução é possível usar o teorema de Cauchy que estabelece quais são as condições devem ter a equação para ser resolvida, no entanto o teorema estabelece somente a existência da solução, para as equações que podem ser resolvidas por métodos analíticos, a técnica analítica denominada método das características tem a particularidade que pode mudar para ser utilizada como uma técnica numérica. 


\subsection{Equação Diferencial Parcial Implícita de Primeira Or- dem}

Uma equação diferencial parcial de primeira ordem em duas variáveis denota-se por

$$
F\left(x, y, u, u_{x}, u_{y}\right)=0
$$

tal que $F \in C^{2}(\Omega)$, onde $\Omega \subset \mathbf{R}^{2} \times \mathbf{R} \times \mathbf{R}^{2}$ domínio aberto, tal que é aplicável o teorema da função implícita com respeito a $u_{x}$ ou $u_{y}$.

Denotando por $p=u_{x}, q=u_{y}$ para simplificar a notação, obtemos alternativamente

$$
F(x, y, u, p, q)=0
$$

onde a função $u$ esta definida como $u: V \rightarrow \mathbf{R}, V \subset \mathbf{R}^{2}$ aberto, e o conjunto $\Omega^{*}$ é projeção de $\Omega$ sobre $\mathbf{R}^{3}$ dada por $(x, y, u)$. Assim, pode-se imaginar em $\Omega^{*}$ que o gráfico de $u$ é o conjunto de pontos que tem em cada ponto o vetor normal $(p, q,-1)$ como na Figura 1.2(b). Esta interpretação propõe o seguinte: por cada vetor tangente a uma curva inicial dada e seu vetor normal à superfície, podemos gerar soluções características da função $u$.

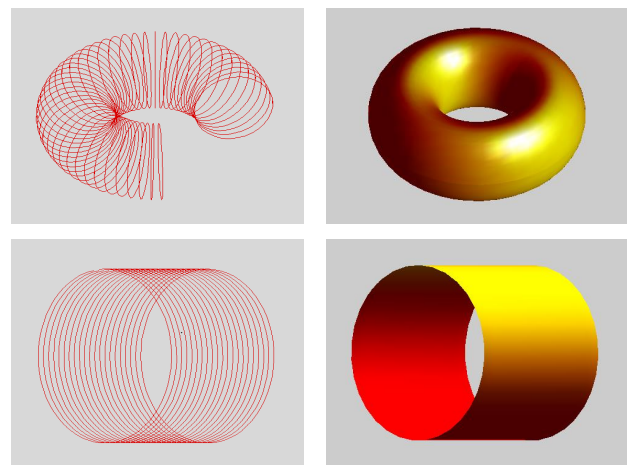

(a) Envoltorias

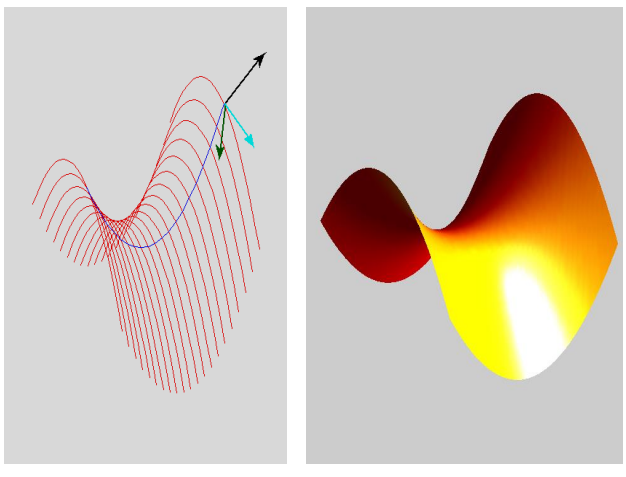

(b) Curvas características

Figura 1.2: Método da envoltória e curvas características

Com o objetivo de estabelecer o teorema de Cauchy generalizado usaremos também a seguinte notação

$$
F\left(x_{1}, x_{2}, u, u_{x_{1}}, u_{x_{2}}\right)=0
$$

a sua vez, denotando por $p_{1}=u_{x_{1}}, p_{2}=u_{x_{2}}$ obtemos

$$
F\left(x_{1}, x_{2}, u, p_{1}, p_{2}\right)=0
$$

o vetor normal ficaria como $\left(p_{1}, p_{2},-1\right)$ 


\subsection{Classificação das Equações Diferenciais Parciais de Primeira Ordem}

Dada uma equação diferencial parcial de primeira ordem em duas variáveis

$$
F\left(x, y, u, u_{x}, u_{y}\right)=0
$$

segundo a forma da equação pode-se classificar nos seguintes tipos :

- Equação Diferencial Parcial Linear: Uma EDP de primeira ordem será denominada linear, se pode expressar-se na forma

$$
R_{1}(x, y) u_{x}+R_{2}(x, y) u_{y}=T(x, y, u)
$$

ou em forma equivalente

$$
F\left(x, y, u, u_{x}, u_{y}\right)=R_{1}(x, y) u_{x}+R_{2}(x, y) u_{y}-T(x, y, u)=0
$$

- Equação Diferencial Parcial Quase-linear: Uma EDP de primeira ordem será denominada quase-linear se pode expressar-se como

$$
R_{1}(x, y, u) u_{x}+R_{2}(x, y, u) u_{y}=T(x, y, u)
$$

ou em forma equivalente

$$
F\left(x, y, u, u_{x}, u_{y}\right)=R_{1}(x, y, u) u_{x}+R_{2}(x, y, u) u_{y}-T(x, y, u)=0
$$

- Equação Diferencial Parcial Não-linear: Se uma EDP de primeira ordem não é linear, nem quase-linear, a equação denomina-se não linear.

Exemplo 1.1 As seguintes equações representam casos particulares das definições estabelecidas

$$
\begin{array}{ccccc}
u_{x}+\left(x y^{3}\right) u_{y}=x u^{2} & \text { ou } & F\left(x, y, u, u_{x}, u_{y}\right)=u_{x}+\left(x y^{3}\right) u_{y}-x u^{2}=0 & \text { Equação linear } \\
2 y u_{x}+u u_{y}=u x^{2} & \text { ou } & F\left(x, y, u, u_{x}, u_{y}\right)=2 y u_{x}+u u_{y}-u x^{2}=0 & \text { Equação quase-linear } \\
y u_{x}+\left(u_{y}\right)^{2}=x y u & \text { ou } & F\left(x, y, u, u_{x}, u_{y}\right)=y u_{x}+\left(u_{y}\right)^{2}-x y u=0 & \text { Equação não linear }
\end{array}
$$


Analogamente, no caso geral, dada uma equação diferencial parcial de primeira ordem de $n$ variáveis

$$
F\left(x_{1}, x_{2}, \ldots, x_{n}, u, u_{x_{1}}, u_{x_{2}}, \ldots, u_{x_{n}}\right)=0
$$

denotando por $x=\left(x_{1}, x_{2}, \ldots, x_{n}\right)$, de acordo com a forma da equação (1.5) podemos definir as Equações Diferencias Lineares e Quase-lineares como segue:

\section{Equação Linear}

$$
\sum_{i=1}^{n} R_{i}(x) u_{x_{i}}=T(x, u)
$$

\section{Equação Quase-linear}

$$
\sum_{i=1}^{n} R_{i}(x, u) u_{x_{i}}=T(x, u)
$$

Se a equação (1.5) não for linear nem quase-linear,a equação denomina-se não-linear. De forma equivalente, ao considerar a notação $p=\left(p_{1}, p_{2}, \ldots, p_{n}\right)$, onde $p_{i}=u_{x_{i}} \operatorname{com} i=1,2, \ldots, n$, uma equação linear estaria definida por

$$
F(x, u, p)=\sum_{i=1}^{n} R_{i}(x) p_{i}-T(x, u)=0
$$

e uma equação quase-linear estaria definida por

$$
F(x, u, p)=\sum_{i=1}^{n} R_{i}(x, u) p_{i}-T(x, u)=0
$$




\subsection{Solução de uma Equação Diferencial Parcial de Pri- meira Ordem}

Definição 1.1 Uma função $\phi: V \rightarrow \mathbf{R}$ definida num aberto $V \subset \mathbf{R}^{2}$ é uma solução da equação (1.1) se verifica:

$$
\left\{\begin{array}{l}
\phi \in C^{2}(V) \\
F\left(x, y, \phi(x, y), \phi_{x}(x, y), \phi_{y}(x, y)\right)=0 \quad \forall(x, y) \in V
\end{array}\right.
$$

os tipos de solução analítica podem-se classificar como: Solução geral, Solução completa, Solução particular e Solução envoltória (Espindola, 2014). A continuação damos um exemplo que explique a diferença entre estes tipos de solução.

Exemplo 1.2 A EDP de primeira ordem linear $y u_{x}-x u_{y}=0$, tem como solução geral a função $u=\phi\left(x^{2}+y^{2}\right)$ notemos que uma solução geral depende de uma função arbitraria, desta solução podemos estabelecer uma solução completa

$$
x^{2}+y^{2}+(u-a)^{2}=1
$$

as soluções completas dependem de constantes arbitrárias, como uma solução particular é independente de constantes o funções arbitrárias se damos um valor à constante $a$ obtemos uma solução particular

$$
x^{2}+y^{2}+(u-3)^{2}=1
$$

uma solução envoltória envolve outras soluções que satisfazem a EDP original, podem-se obter impondo uma condição à solução completa obtida, por exemplo a $x^{2}+y^{2}=1$ é uma solução envoltória pois obtêm-se da solução completa da forma $\varphi(x, y, a)$ impondo que $\partial \varphi / \partial a=0$ assim $2(u-a)=0$.

De acordo como mencionamos na introdução existem diferentes técnicas analíticas que resolvem os casos em que a equação é linear, quase-linear e não linear, nos desenvolveremos o método das características, que permitirá mas para enfrente mudar a um método numérico. 


\subsection{Teoria sobre as curvas características}

Dada uma EDP de primeira ordem implícita

$$
F\left(x, y, u, u_{x}, u_{y}\right)=0
$$

denotando por, $u=f(x, y)$, é possível estabelecer intuitivamente a seguinte ideia geométrica, se tivermos inicialmente uma curva integral, o gráfico dela é uma curva contida no gráfico da superfície, poderíamos imaginar a possibilidade de parametrizar essa curva e para cada valor do parâmetro, determinar outras curvas integrais cujos gráficos também estarão contidos na superfície. Se todas estas curvas integrais fossem parametrizáveis, obteríamos uma solução paramétrica, usando uma "curva inicial" (Figura1.3). Estabeleceremos agora o método das características, que permite determinar curvas integrais as quais denominaremos logo como "curvas características".

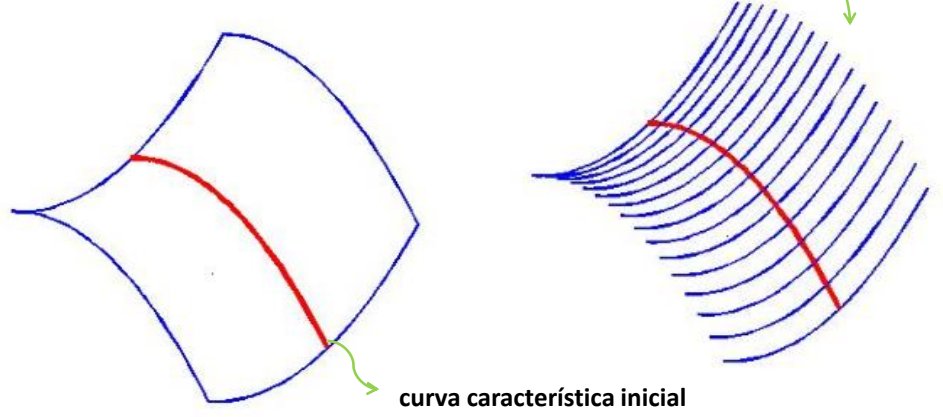

Figura 1.3: Curvas características

Estabeleceremos agora a definição de curva característica.

Definição 1.2 Se uma equação diferencial parcial esta determinada implicitamente por:

$$
F\left(x, y, u, u_{x}, u_{y}\right)=0
$$

Sendo $u=f(x, y)$, e supondo que a curva $\sigma: I \subset \mathbf{R} \rightarrow \mathbf{R}^{5}$ é definida parametricamente por $\sigma(t)=(X(t), Y(t), U(t), P(t), Q(t))$ onde $x=X(t), y=Y(t), U(t)=u(X(t), Y(t))$, sendo $p=u_{x}, q=u_{y}$ temos que $p(X(t), Y(t))=P(t), q(X(t), Y(t))=Q(t)$, esta curva $\sigma$ assim definida, está contida na superfície solução, ou seja, verifica que $F(\sigma(t))=0$. A projeção dos pontos de $\sigma(t)$ sobre $\mathbf{R}^{3}$ dada por $(X(t), Y(t), U(t))$, com vetor normal $N=(P(t), Q(t),-1)$, define uma curva, a qual denomina-se, curva solução ou "curva característica” (Figura 1.4). 


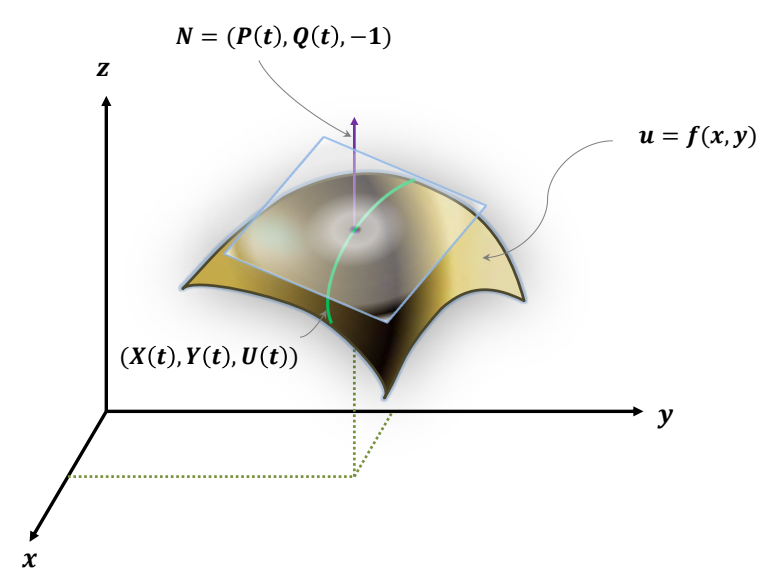

Figura 1.4: Curva característica parametrizada

\subsection{Solução de EDPs Lineares usando o método das ca- racterísticas}

Se consideramos uma EDP linear de primeira ordem

$$
R_{1}(x, y) u_{x}+R_{2}(x, y) u_{y}=T(x, y, u)
$$

para poder estabelecer um método de solução desta equação, temos que as funções $R_{1}, R_{2}, T$ precisam de algumas condições, pois se, por exemplo, $R_{1} \equiv 0$, e $R_{2} \equiv 0$, não existiria uma equação em derivadas parciais.

Para dar uma ideia de como estabelecer ditas condições para as funções $R_{1}, R_{2}, T$, consideraremos o caso particular

$$
R_{1}(x, y) u_{x}+R_{2}(x, y) u_{y}=T(x, y)
$$

ou equivalentemente $F\left(x, y, u, u_{x}, u_{y}\right)=R_{1}(x, y) u_{x}+R_{2}(x, y) u_{y}-T(x, y)=0$.

A equação (1.8) pode ser descrita como

$$
\left(R_{1}(x, y), R_{2}(x, y), T(x, y)\right) \cdot\left(u_{x}(x, y), u_{y}(x, y),-1\right)=0
$$

daqui supondo que a função $u$ é suave, o vetor normal à superfície num ponto $(x, y, u(x, y))$, denotado por $N(x, y)=\left(u_{x}(x, y), u_{y}(x, y),-1\right)$, é ortogonal ao vetor $\left(R_{1}(x, y), R_{2}(x, y), T(x, y)\right)$, em cada ponto $(x, y)$ do domínio da função $u$. Se $u: V \rightarrow \mathbf{R}, V \subset \mathbf{R}^{2}$ aberto, temos que $R_{1}, R_{2}, T$ devem estar definidas em $V$, e mais ainda existirá uma vizinhança do ponto $(x, y, u(x, y))$ tal que, 
para cada curva, que passe por este ponto, o vetor tangente à curva nesse ponto, deve estar contido no plano tangente à superfície como na Figura 1.5(a), sempre que a curva esteja contida na interseção da vizinhança e a superfície.

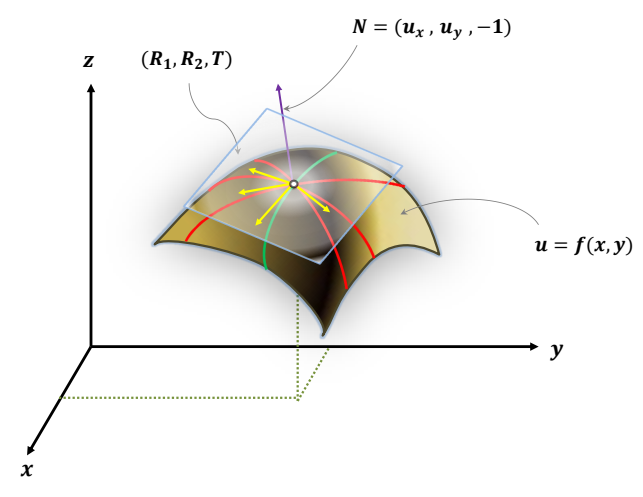

(a) Plano tangente à superficie $u=f(x, y)$

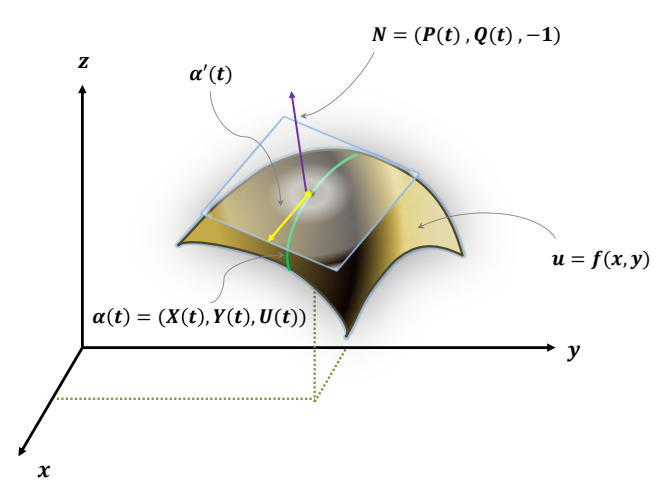

(b) Plano tangente à Curva característica inicial

Figura 1.5: Relação da Curva característica com o plano tangente

se consideramos que sobre a superfície solução, descrita como na definição de curva característica (Definição 1.2), acha-se a curva paramétrica

$$
(X(t), Y(t), U(t))
$$

onde $U(t)=u(X(t), Y(t))$, sendo $\alpha: J \rightarrow \mathbf{R}^{3}, J \subset \mathbf{R}$ e $\alpha(t)=(X(t), Y(t), U(t))$, como na Figura 1.5(b) é possível encontrar uma solução local (Ireneo, 1995; Rhee e Amundson, 1986) estabelecendo o seguinte, notamos que $\alpha^{\prime}(t)$ deve estar contido no plano tangente determinado pelo vetor normal $N=(P(t), Q(t),-1)$, assim $\alpha^{\prime}(t)=k\left(R_{1}(X(t), Y(t)), R_{2}(X(t), Y(t)), T(X(t), Y(t))\right)$.

Sendo $u$ suave, existirá uma curva paramétrica $\beta(s)=(X(s), Y(s), U(s))$ que passa pelo ponto de tangência da curva $\alpha(t)$, como nas Figuras 1.6(a), 1.6(b) o vetor tangente $\beta^{\prime}(s)$ correspondente ao ponto de tangencia, também deve estar contido no plano tangente, $\operatorname{logo} \beta^{\prime}(s)=$ $m\left(R_{1}(X(s), Y(s)), R_{2}(X(s), Y(s)), T(X(s), Y(s))\right)$, assim em particular a curva $\beta(s)$ deve satisfazer o sistema

$$
\left\{\begin{aligned}
\frac{d X}{d s} & =R_{1}(X(s), Y(s)) \\
\frac{d Y}{d s} & =R_{2}(X(s), Y(s)) \\
\frac{d U}{d s} & =T(X(s), Y(s))
\end{aligned}\right.
$$

As equações

$$
\left\{\begin{aligned}
\frac{d X}{d s} & =R_{1}(X(s), Y(s)) \\
\frac{d Y}{d s} & =R_{2}(X(s), Y(s))
\end{aligned}\right.
$$


podem-se resolver no plano $x y$, estas equações determinam uma família de curvas $(X(s), Y(s))$ com $x=X(s), y=Y(s)$, as curvas obtidas denominam-se "curvas características base"

A equação

$$
\frac{d U}{d s}=T(X(s), Y(s))
$$

pode ser resolvida substituindo as soluções das equações (1.11), para obter a curva $(X(s), Y(s), U(s))$ que é uma "curva característica", como na Figura 1.6(a), por outro lado, se por cada ponto da curva parametrizada (1.9) passa uma curva caraterística, a família dessas características pode-se escrever como

$$
\Gamma(s, t)=(X(s, t), Y(s, t), U(s, t))
$$

Considerando $x=X(s, t), y=Y(s, t), u=U(s, t)$, a curva inicial corresponde ao valor de $s=0$, sempre que, $\Gamma(0, t)=(X(0, t), Y(0, t), U(0, t))=\alpha(t)$, assim (1.13) representa a parametrização da superfície que contém a curva paramétrica (1.9), sendo $X(0, t)=X(t), Y(0, t)=$ $Y(t), U(0, t)=U(t)$

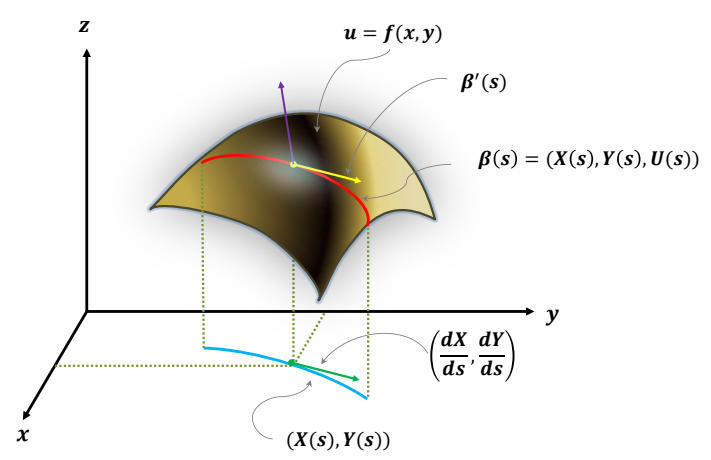

(a) Curva característica inicial

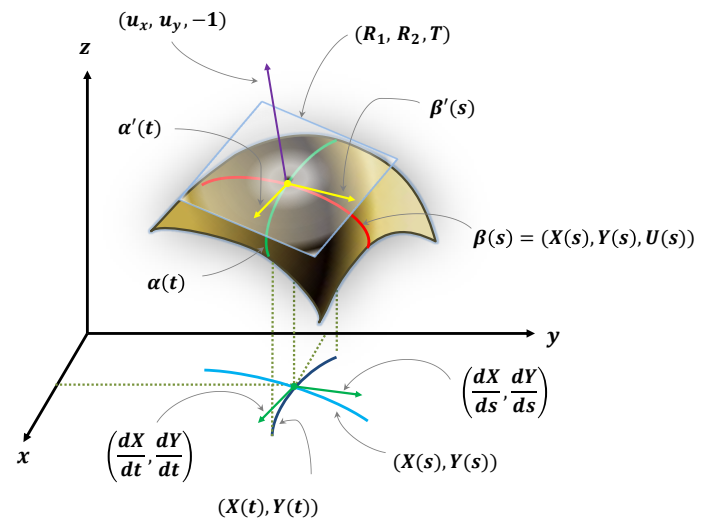

(b) Curvas características

Figura 1.6: Relação entre a solução de uma EDP e seus curvas características

No sistema (1.10) ao impor as condições iniciais $X\left(0, t_{0}\right)=x_{0}, Y\left(0, t_{0}\right)=y_{0}, U\left(0, t_{0}\right)=u_{0}$, o problema fica descrito como

$$
\left\{\begin{aligned}
\frac{d X}{d s} & =R_{1}(X(s), Y(s)) & & X(0)=x_{0} \\
\frac{d Y}{d s} & =R_{2}(X(s), Y(s)) & & Y(0)=y_{0} \\
\frac{d U}{d s} & =T(X(s), Y(s), U(s)) & & U(0)=u_{0}
\end{aligned}\right.
$$

poderíamos obter uma solução particular $U=u(X(s, t), Y(s, t))$ como na Figura 1.7 que contém a curva solução inicial (1.9), no entanto pode acontecer que o sistema de equações tenha infinitas soluções ou não tenha solução, porém para que as curvas $\beta(s)$ e $\alpha(t)$ sejam transversais devemos exigir que 


$$
J=\frac{\partial(X, Y)}{\partial(s, t)}=\left|\begin{array}{cc}
X_{s} & Y_{s} \\
X_{t} & Y_{t}
\end{array}\right| \neq 0
$$

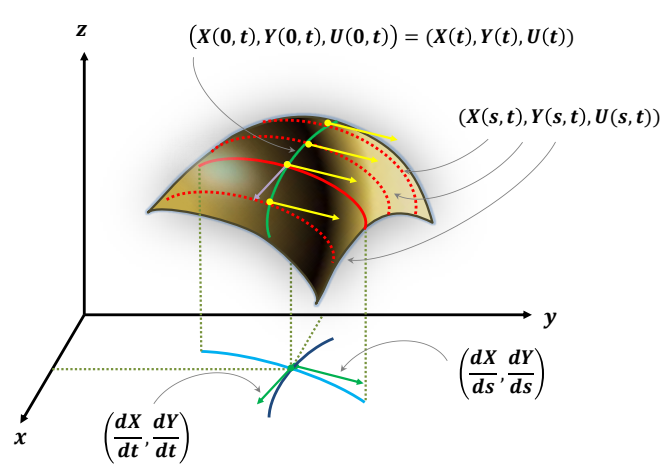

Figura 1.7: Solução paramétrica

Portanto, para encontrar a solução de uma EDP linear de primeira ordem poderíamos resolver um sistema de EDOs não linear de primeira ordem, supondo que as funções $R_{1}, R_{2}, T$ da equação (1.8), sejam definidas em $\Omega^{*} \subset \mathbf{R}^{2}$, tal que $V \subset \Omega^{*}$ sendo $u: V \rightarrow \mathbf{R}, V \subset \mathbf{R}^{2}$ aberto, também

i) $R_{1}, R_{2}, T \in \mathbf{C}^{1}\left(\Omega^{*}\right)$

ii) $\left|R_{1}(x, y)\right|+\left|R_{2}(x, y)\right|>0$ para $(x, y) \in V$

assim pelo teorema de Picard o sistema (1.14) tem solução única. Uma ideia geométrica para determinar a solução numérica da curva $\beta(s)$, usando o método da projeção, que será desenvolvido no Capítulo 3, é dada pelo gráfico da Figura 1.8

Por outro lado, dado um sistema de equações

$$
x^{\prime}=g(t, x), \quad x\left(t_{0}\right)=x_{0}
$$

é possível garantir a existência e unicidade da solução numa vizinhança de $t_{0}$ sempre que a função $g: \mathbf{R} \times \mathbf{R}^{n} \rightarrow \mathbf{R}^{n}$ seja contínua e localmente Liptchitziana. 


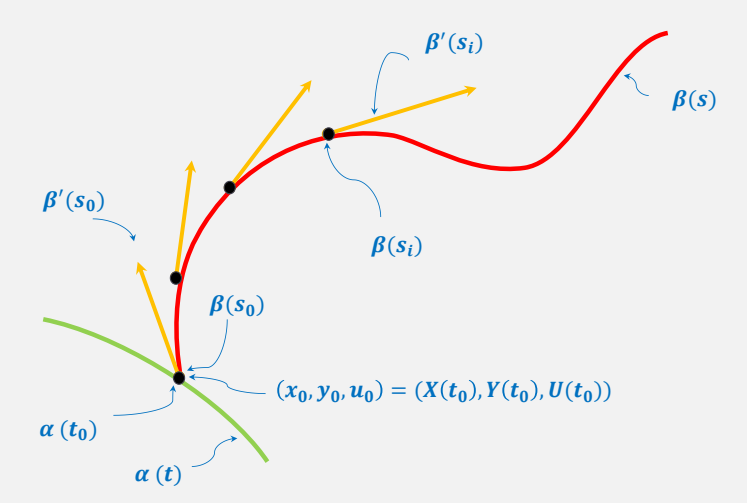

Figura 1.8: Curva caraterística gerada pela curva característica inicial

\section{A Equação do Transporte}

Resolveremos agora um caso particular EDPs lineares de primeira ordem, denominada a equação do transporte a qual esta dada por

$$
\begin{gathered}
a u_{x}+u_{y}=0 \\
u(x, 0)=f(x)
\end{gathered}
$$

onde $a$ é un número real constante que satisfaz a condição inicial.

Ao parametrizar a curva inicial, a curva ficaria definida por $(X(t), Y(t), U(t))=(t, 0, f(t))$ e as equações características estariam dadas por

$$
\left\{\begin{array}{l}
\frac{d X}{d s}=a \\
\frac{d Y}{d s}=1 \\
\frac{d U}{d s}=0
\end{array}\right.
$$

cuja solução está dada por

$$
\left\{\begin{array}{l}
X(s)=a s+c_{1} \\
Y(s)=s+c_{2} \\
U(s)=c_{3}
\end{array}\right.
$$

assim

$$
\left\{\begin{array}{l}
t=X(0, t)=X(0)=c_{1} \\
0=Y(0, t)=Y(0)=c_{2} \\
f(t)=U(0, t)=U(0)=c_{3}
\end{array}\right.
$$


daqui

$$
\left\{\begin{array}{l}
X(s, t)=a s+t \\
Y(s, t)=s \\
U(s, t)=f(t)
\end{array}\right.
$$

e como $x=X(s, t), y=Y(s, t)$, obtemos que $t=x-a y$, portanto $u(x, y)=f(x-a y)$

Num caso particular em que $a=0.5$, e para a função $f(x)=\exp \left(-x^{2}\right)$ obtemos a gráfica das Figuras 1.9(a), 1.9(b)

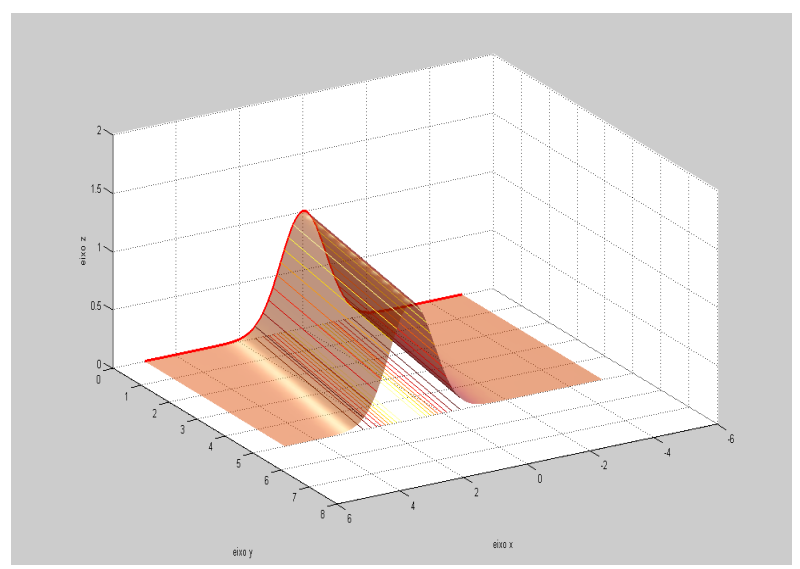

(a) Linhas características

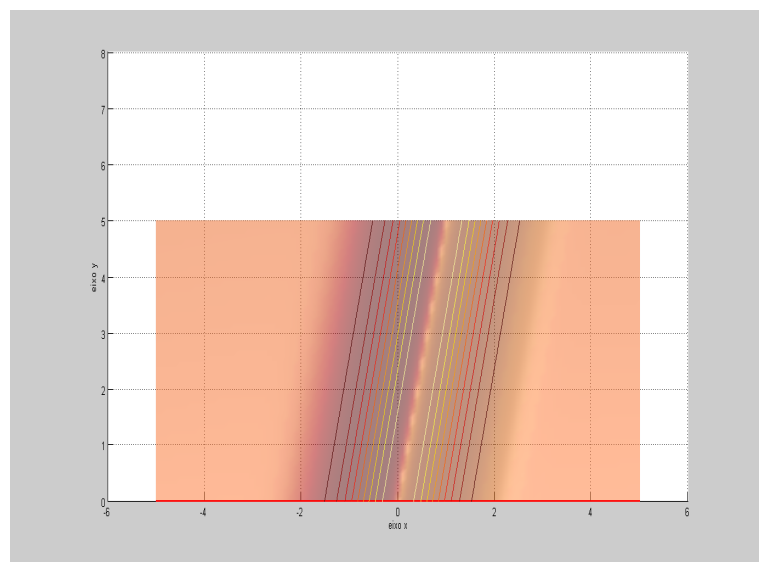

(b) Linhas características base

Figura 1.9: Solução $u(x, y)=f(x-0.5 y)$

Podemos notar que a forma da onda não muda para os diferentes valores quando $y$ aumenta, e como a solução tem a forma $u(x, y)=f(x-a y)$ ao mudar a forma da função $f(x)$ o efeito seria o mesmo, a sua vez dependendo do valor da constante os deslocamentos serão para a direita se $a>0$ e à esquerda se $a<0$

É importante ressaltar o efeito da onda viajante, para cada curva característica $t=x-$ ay o valor da função $u$ é constante, porém todos os dados iniciais transmitem-se ao longo das curvas características base e propagam-se ao longo destas curvas provocando o efeito viajante na solução da equação, vejamos alguns exemplos donde mudaremos só a condição inicial $u(x, 0)=f(x)$

Exemplo 1.3 Para a função

$$
\begin{gathered}
f(x)=\left\{\begin{array}{c}
0 \quad,|x|>1 \\
1-x^{2} \quad,|x| \leq 1
\end{array}\right. \\
u(x, 0)=f(x)
\end{gathered}
$$

notamos a propagação de ondas ao longo das curvas caraterísticas base Figuras 1.10(a), 1.10(b) 


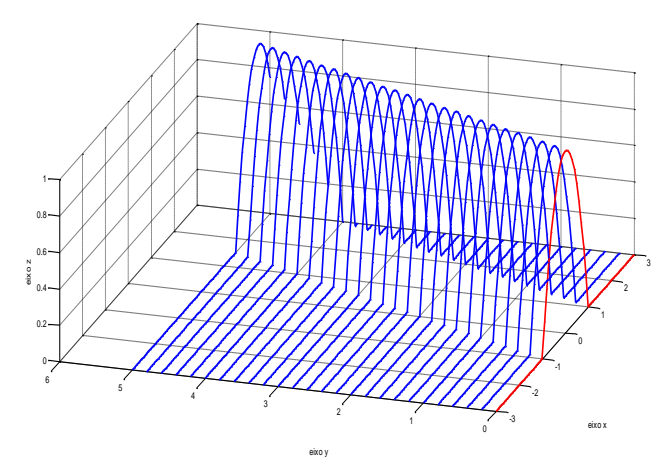

(a) Curvas características vista desde o eixo y

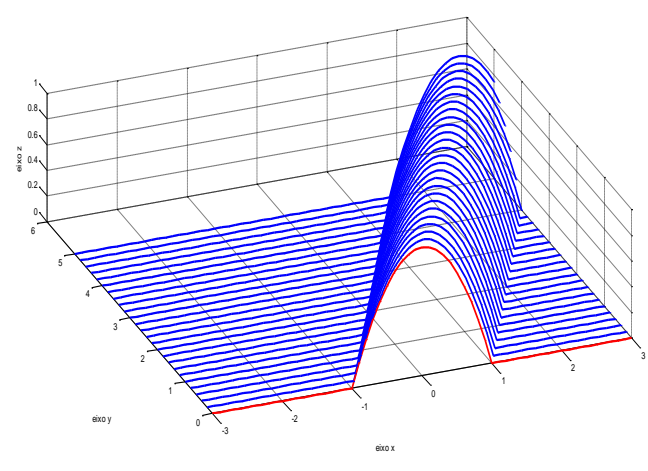

(b) Curvas características vista desde o eixo $\mathrm{x}$

Figura 1.10: Ondas viajantes parabólicas

Exemplo 1.4 Ao considerar a função delta de Dirac, que definida em forma aproximada estaria dada por $\delta_{n}(x)=\frac{n}{\sqrt{\pi}} \exp \left(-n^{2} x^{2}\right)$

$$
u(x, 0)=\delta_{n}(x)
$$

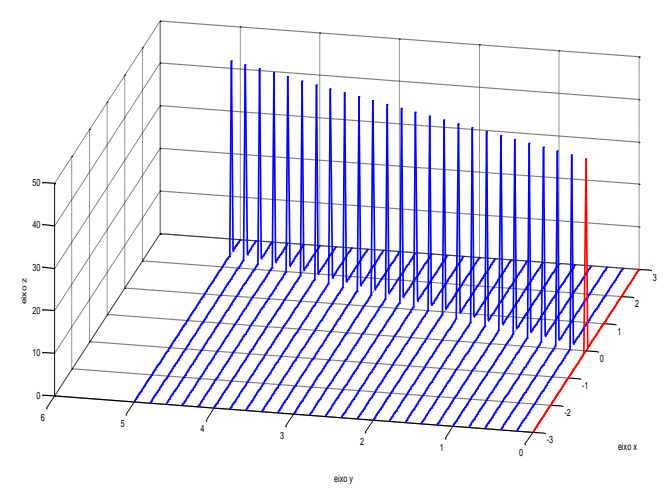

(a) Curvas características vista desde o eixo y

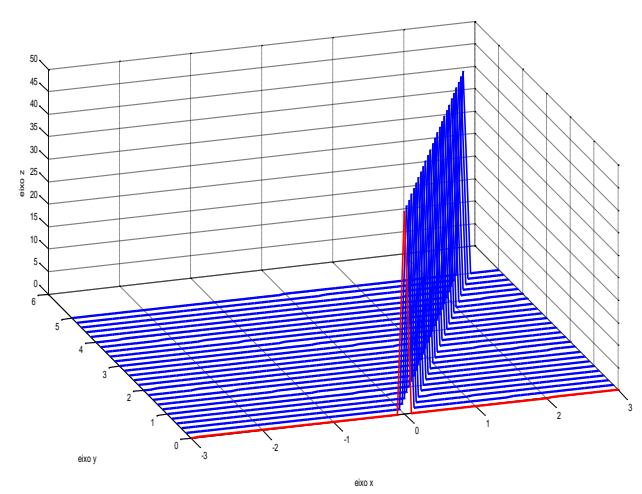

(b) Curvas características vista desde o eixo $\mathrm{x}$

Figura 1.11: Ondas viajantes delta Dirac

podemos notar a propagação de uma perturbação Figuras 1.11(a), 1.11(b)

Exemplo 1.5 A propagação de descontinuidades pode-se representar, considerando a função de Heaviside $H(x)$ definida por

$$
\begin{gathered}
H(x)= \begin{cases}1 & , x>0 \\
0 & , x \leq 0\end{cases} \\
u(x, 0)=H(x+1)-H(x-1)
\end{gathered}
$$




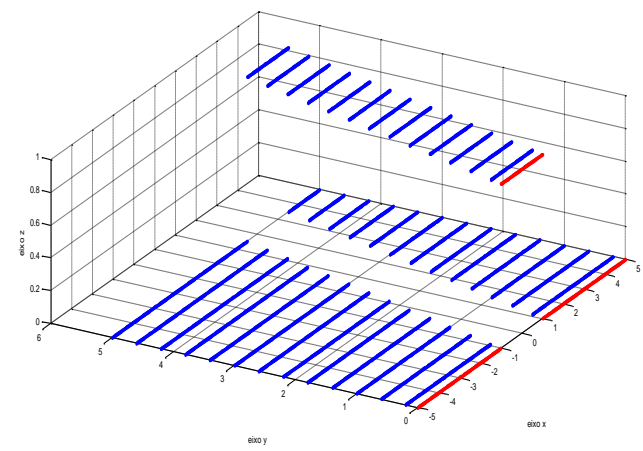

(a) Curvas características vista desde o eixo y

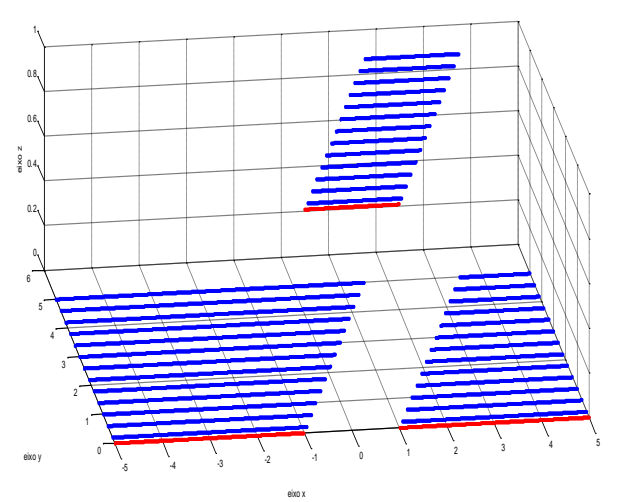

(b) Curvas características vista desde o eixo $\mathrm{x}$

Figura 1.12: Ondas viajantes descontinuas

a propagação da descontinuidade mostra-se nas Figuras 1.12(a), 1.12(b)

Exemplo 1.6 Para mostrar a propagação de não diferenciabilidade, consideraremos a função definida por

$$
\begin{gathered}
f(x)=\left\{\begin{array}{cc}
0 \quad,|x|>1 \\
1-|x| \quad,|x| \leq 1
\end{array}\right. \\
u(x, 0)=f(x)
\end{gathered}
$$

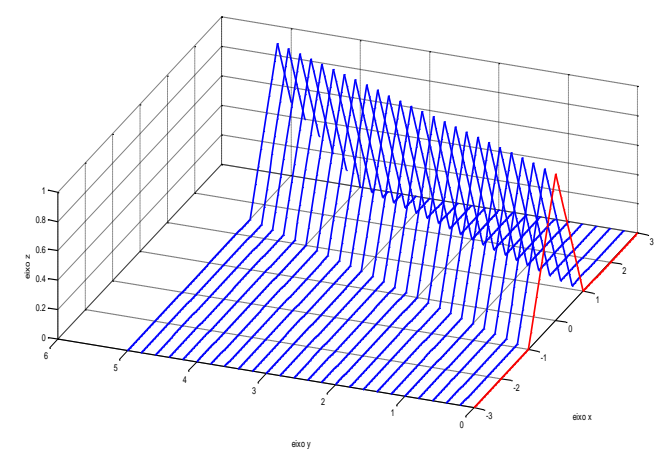

(a) Curvas características vista desde o eixo y

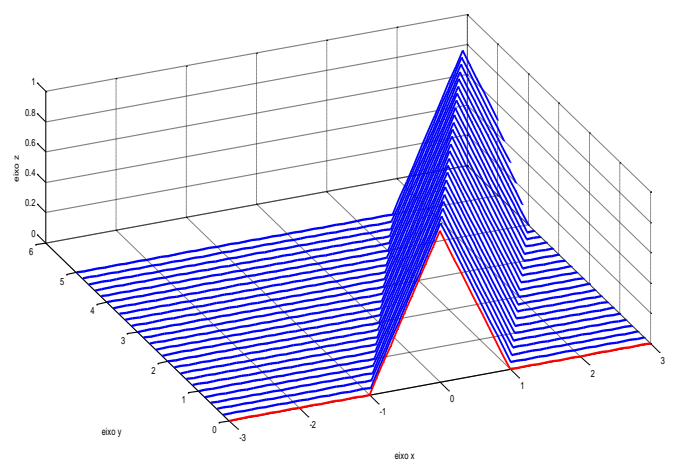

(b) Curvas características vista desde o eixo $\mathrm{x}$

Figura 1.13: Ondas viajantes não diferenciáveis

notar a propagação da onda viajante nas Figuras 1.13(a), 1.13(b).

No caso que os coeficientes não sejam constantes dependendo deles e o grau de complexidade da solução do sistema característico podemos obter seu solução analítica. 
Exemplo 1.7 Dada a equação

$$
x u_{x}+y u_{y}=-u
$$

sendo uma equação linear da forma

$$
R_{1}(x, y) u_{x}+R_{2}(x, y) u_{y}=T(x, y, u)
$$

temos que

$$
y^{\prime}(x)=\frac{d y}{d x}=\frac{\frac{d Y}{d s}}{\frac{d X}{d s}}=\frac{R_{2}}{R_{1}}
$$

assim nosso caso $y^{\prime}(x)=\frac{y}{x}$ cuja solução permite obter as curvas características $y=k x$

Exemplo 1.8 Dada a equação

$$
\begin{gathered}
x u_{x}+y u_{y}=c u \\
u(x, 1)=f(x) \\
x \in \mathbf{R}, y>0, c=\text { constante }
\end{gathered}
$$

Resolvendo o sistema

$$
\begin{cases}\frac{d X}{d s}=X & , X(0)=t \\ \frac{d Y}{d s}=Y & , Y(0)=1 \\ \frac{d U}{d s}=c U & , U(0)=f(t)\end{cases}
$$

obtemos

$$
\left\{\begin{array}{l}
X(s, t)=t e^{s} \\
Y(s, t)=e^{s} \\
U(s, t)=f(t) e^{c s}
\end{array}\right.
$$

assim $u(x, y)=f\left(\frac{x}{y}\right) y^{c}$, no entanto a solução obtida não é definida em $y=0$, como podemos notar nas Figuras 1.14(a), 1.14(b), também pode-se observar a relação com as curvas características nas Figuras 1.15(a), 1.15(b) 


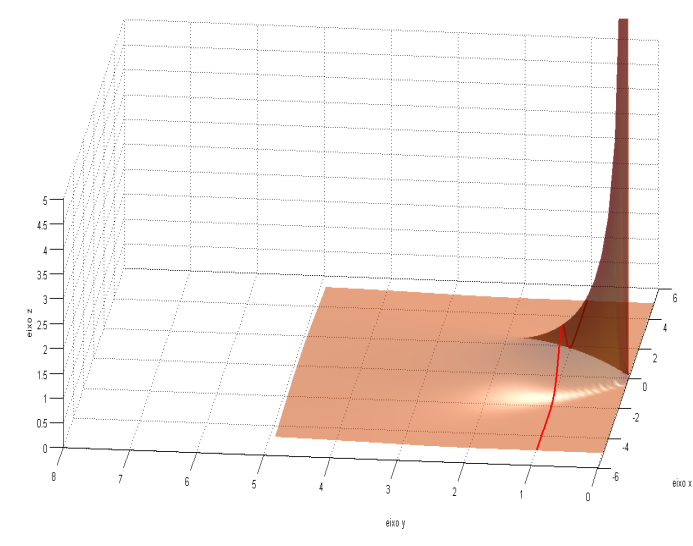

(a) Singularidade vista desde o eixo y

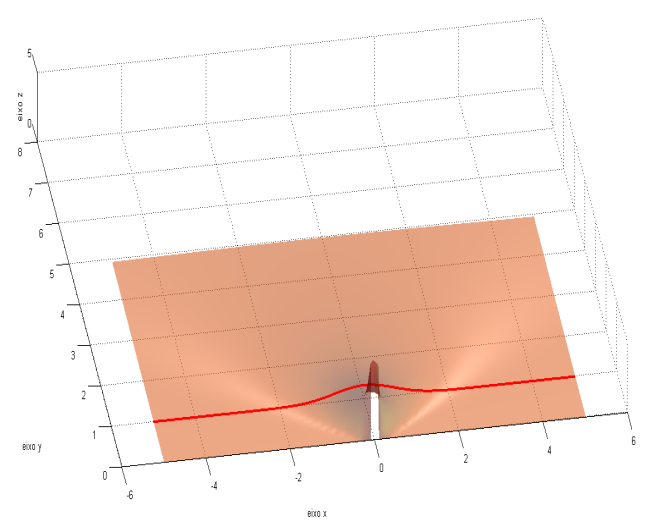

(b) Singularidade vista desde o eixo $\mathrm{x}$

Figura 1.14: Solução da equação $x u_{x}+y u_{y}=c u$ com condição inicial $u(x, 1)=e^{-x^{2}}$ para $c=-1$

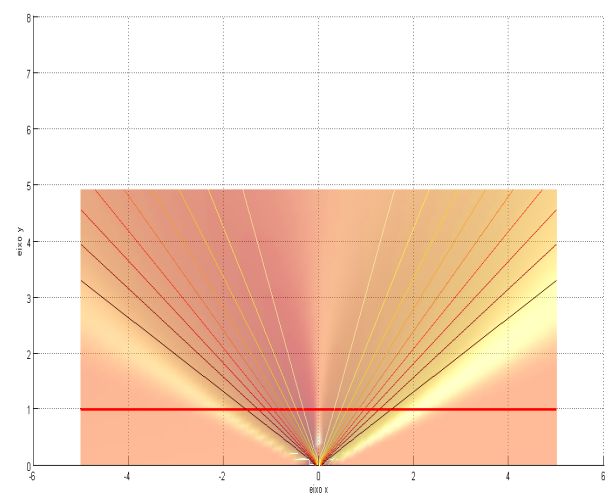

(a) Curvas características base

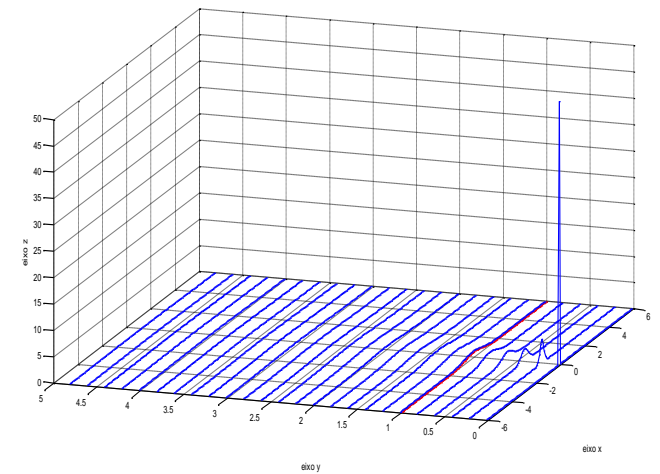

(b) Curvas Características vista desde o eixo $\mathrm{x}$

Figura 1.15: Curvas características e singularidade

Na solução obtida notamos também que seus valores tendem ao infinito quando $(x, y)$ tende a $(0,0)$, tomando a forma indeterminada $\frac{0}{0}$, e que não está definida ao longo da reta $y=0$, porem nestes pontos a função tem uma singularidade como podemos notar nas Figuras 1.16(a), 1.16(b), e as Figuras 1.17(a),1.17(b).

Se para uma curva inicial verifica-se a condição $y=1 \neq 0$ perto dela não existiria dificuldade em gerar as outras curvas características sempre que $c>0$ e que a função $f\left(\frac{y}{x}\right)$ não gere singularidade, no caso que $c<0$ vimos o que acontece, no entanto mesmo sendo $c>0$ poderíamos obter infinitas soluções se para uma curva característica e a curva inicial seus vetores tangentes são paralelos, isto é $J=\frac{\partial(X, Y)}{\partial(s, t)}=0$

Nas equações lineares podemos identificar a forma da equação

$$
R_{1}(x, y) u_{x}+R_{2}(x, y) u_{y}=T(x, y, u)
$$




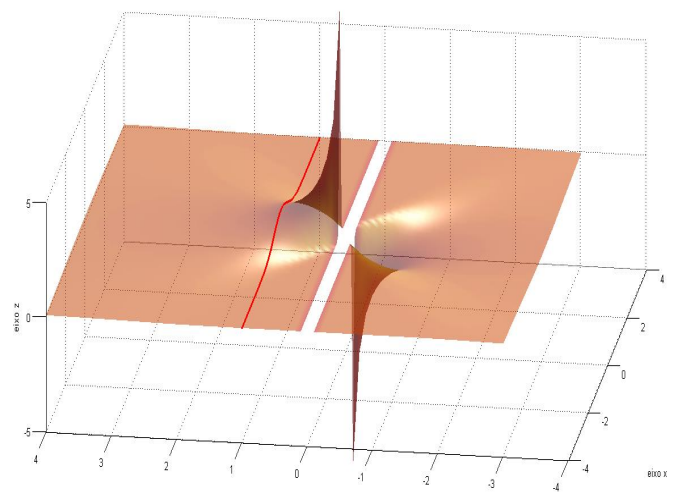

(a) Singularidade vista desde o eixo y

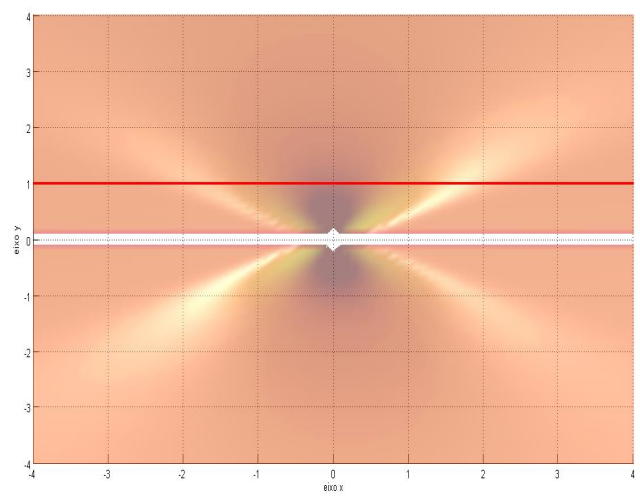

(b) Singularidade vista desde o eixo $\mathrm{x}$

Figura 1.16: Singularidade apresentada na solução da equação $x u_{x}+y u_{y}=c u$ com condição inicial $u(x, 1)=e^{-x^{2}}$ para $c=-1$

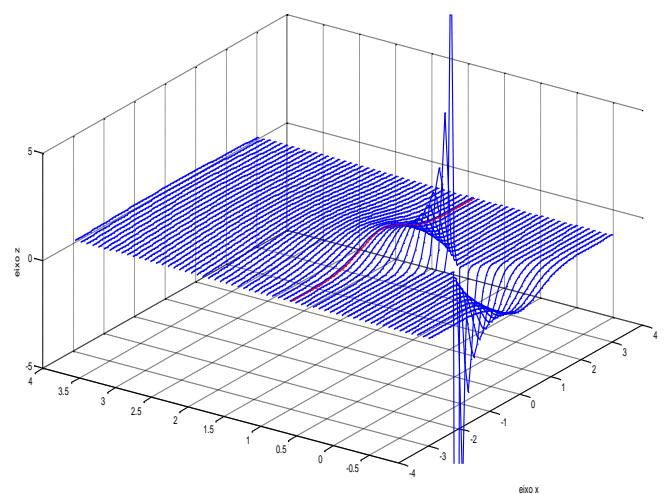

(a) Curvas características vista desde o eixo y

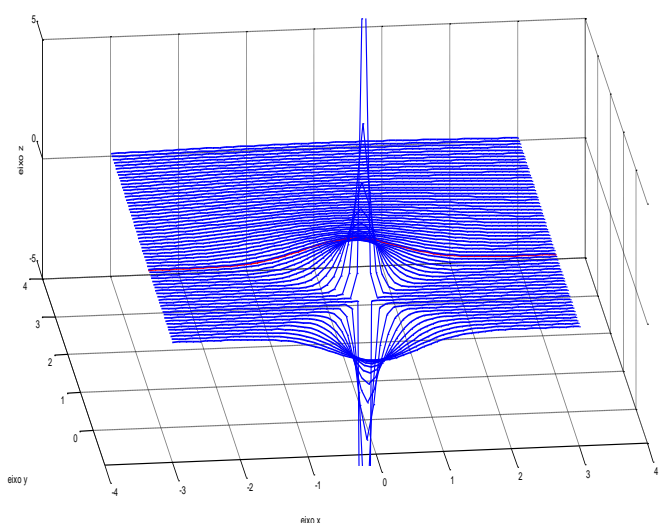

(b) Curvas Características vista desde o eixo $\mathrm{x}$

Figura 1.17: Singularidade e Curvas características

como um produto interior

$$
\left(R_{1}, R_{2}\right) \cdot\left(u_{x}, u_{y}\right)=T(x, y, u)
$$

daqui

$$
\nabla u \cdot v=T(x, y, u)
$$

assim podemos interpretar geometricamente a forma da equação, como a derivada direcional de $u$, que determina a razão de troca ao longo do vetor $v=\left(R_{1}, R_{2}\right)$, porém $T$ determina como é a razão de variação, por exemplo se $T=0$ teremos que $u$ será constante nessa direção. 
Exemplo 1.9 Dada a equação

$$
\begin{gathered}
u_{x}+y u_{y}=0 \\
u(0, y)=f(y)
\end{gathered}
$$

Como a equação é linear tem-se

$$
\begin{cases}\frac{d X}{d s}=1 & X(0)=x_{0} \\ \frac{d Y}{d s}=Y & Y(0)=y_{0}\end{cases}
$$

podemos concluir então que

$$
\frac{d y}{d x}=\frac{\frac{d Y}{d s}}{\frac{d X}{d s}}=Y(s)=y
$$

e devido ao fato que a solução deve passar pelo ponto $\left(x_{0}, y_{0}\right)$, as curvas características estão dadas por $y=y_{0} e^{x-x_{0}}$, logo usando a condição inicial $u(0, y)=f(y)$, obtemos finalmente a solução $u(x, y)=f\left(y e^{-x}\right)$, podemos ver o gráfico da solução na Figura 1.18(a), para o caso particular de $f(y)=\exp \left(-y^{2}\right)$

No caso que resolvamos o sistema característico ao longo da curva inicial, temos que

$$
\left\{\begin{array}{rlrl}
\frac{d X}{d s}=1 & X(0)=0 \\
\frac{d Y}{d s}=Y & Y(0)=t \\
\frac{d U}{d s}=0 & U(0)=f(t)
\end{array}\right.
$$

obtendo $X(s)=s$, que é equivalente a $X(s, t)=s$, e $Y(s, t)=t e^{s}$. Por outro lado como $\frac{d U}{d s}=0$, temos que $U(s)=f(t)$ ou equivalentemente $U(s, t)=f(t)$, das soluções obtidas nas primeiras equações obtemos $t=Y(s, t) e^{-X(s, t)}$, daqui $U(s, t)=f\left(Y(s, t) e^{-X(s, t)}\right)$, sendo $U(s, t)=u(X(s, t), Y(s, t))$ concluímos que $u(x, y)=f\left(y e^{-x}\right)$. Os gráficos das curvas características ao longo da curva inicial estão dados nas Figuras 1.18(b), 1.19(a), 1.19(b)

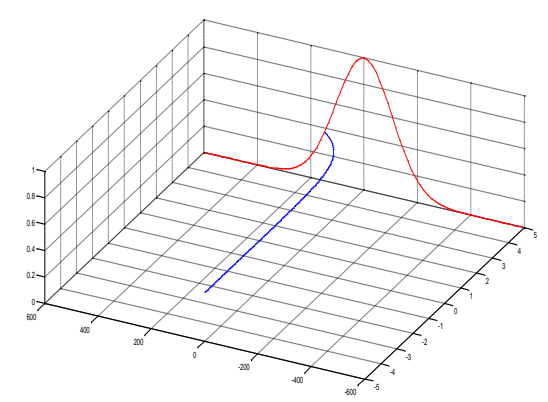

(a) Curva característica e Curva inicial

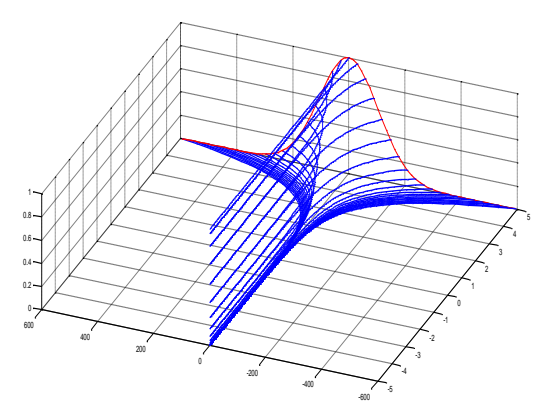

(b) Curvas características

Figura 1.18: Curvas características da equação $u_{x}+y u_{y}=0$, com condição inicial

$$
u(0, y)=e^{-y^{2}}
$$




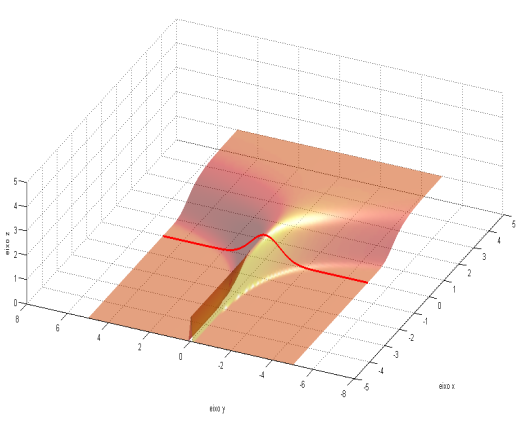

(a) Superficie solução

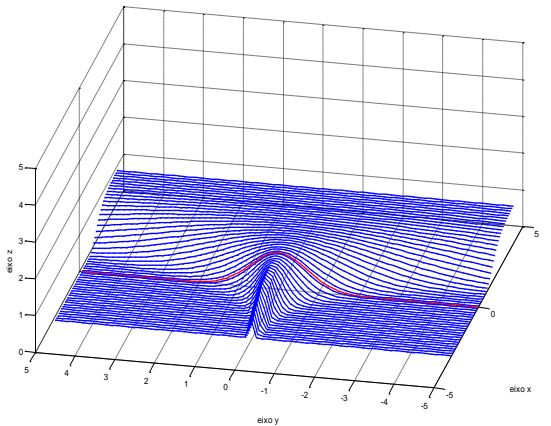

(b) Curvas cararterísticas solução

Figura 1.19: Solução da equação $u_{x}+y u_{y}=0$, com condição inicial $u(0, y)=e^{-y^{2}}$

Exemplo 1.10 Dada a equação

$$
\begin{aligned}
& 2 u_{x}+u_{y}=c u \\
& u(x, 0)=f(x)
\end{aligned}
$$

Para a equação acima, as projeções das curvas características são dadas por $y=\frac{k}{2} x$ no caso em que $c=0$ as curvas características mantêm sua forma ao incrementar os valores de $y$ assim só se desloca progressivamente Figura 1.20(a) mas no caso que $c=1$ os valores da função aumentam conforme aumentam os valores a função $u$ como podemos ver na Figura 1.21(a)

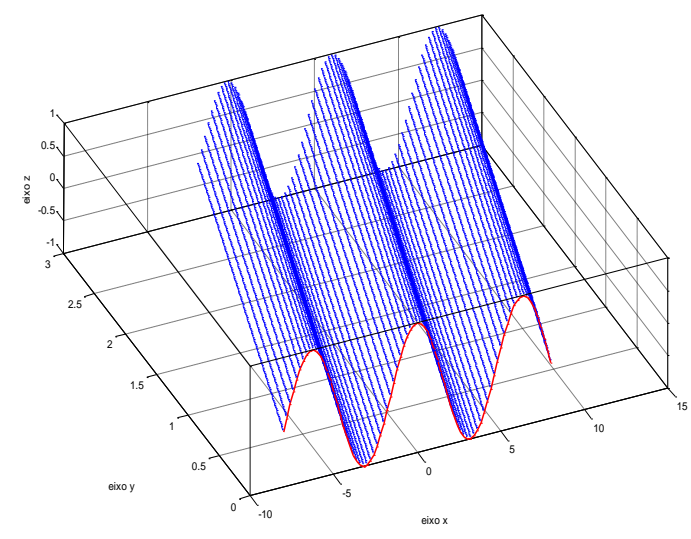

(a) Curvas características e Curva inicial

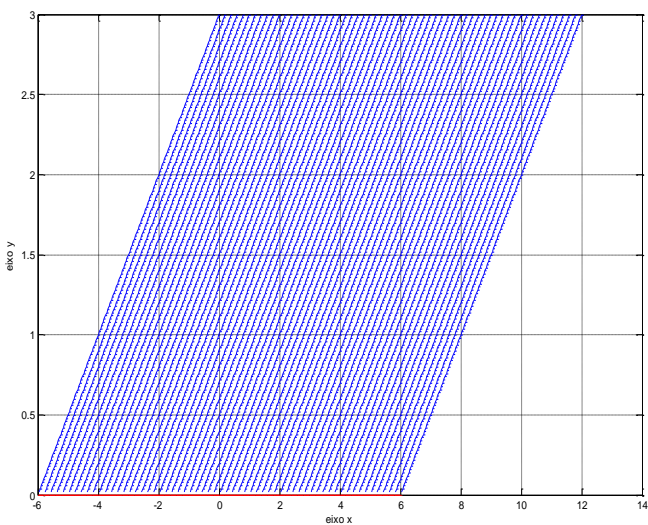

(b) Projeção das características

Figura 1.20: Solução da equação $2 u_{x}+u_{y}=c u, \operatorname{com} c=0$ e condição inicial $u(x, 0)=\cos x$

Exemplo 1.11 Dada a equação

$$
\begin{gathered}
e^{y} u_{x}+u_{y}=c u \\
u(x, 0)=f(x)
\end{gathered}
$$




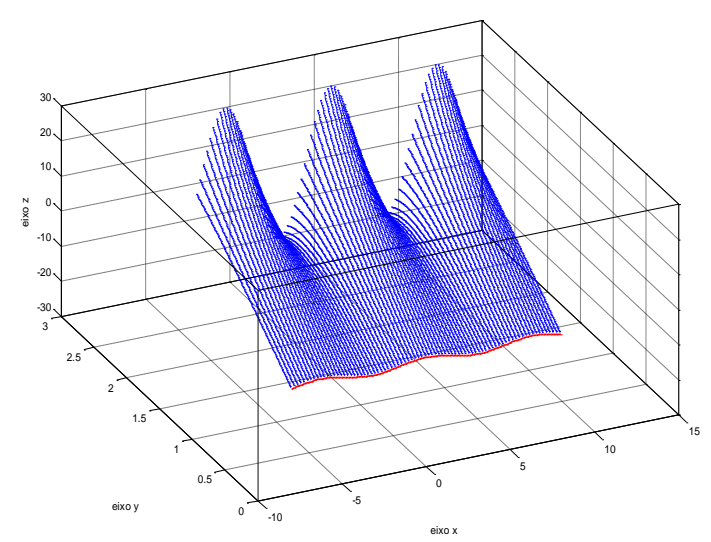

(a) Curvas características e Curva inicial

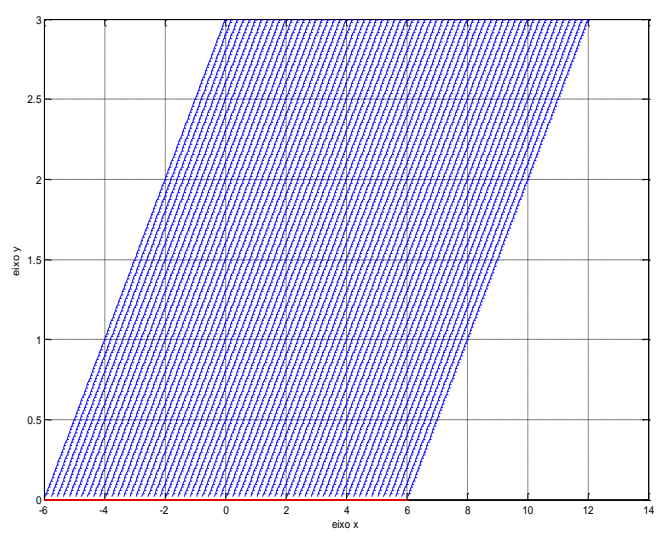

(b) Projeção das curvas características

Figura 1.21: Solução da equação $2 u_{x}+u_{y}=c u, \operatorname{com} c=1$ e condição inicial $u(x, 0)=\cos x$

Para a equação acima as projeções das curvas características são dadas por $y=\ln x+k$ no caso em que $c=0$ as curvas características mantêm sua forma ao incrementar os valores de $y$, assim só se desloca progressivamente como mostra a Figura 1.22(a). Mas no caso que $c=1$, a onda viajante incrementa seu altura conforme incrementem-se os valores da função $u$ como podemos ver na Figura 1.23(a)

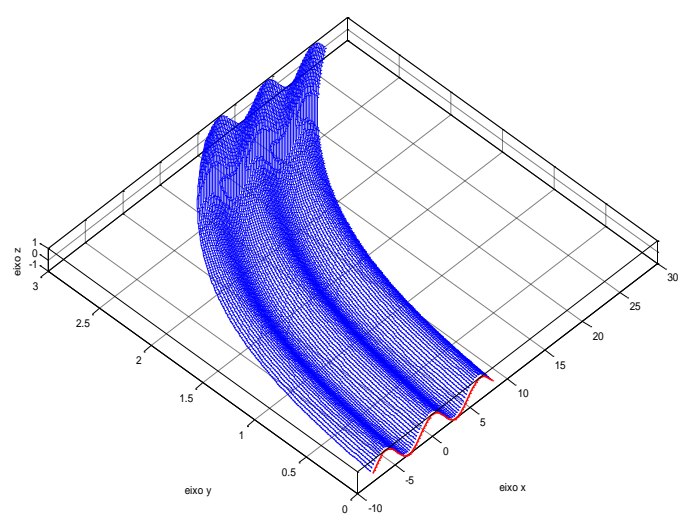

(a) Curvas características e Curva inicial

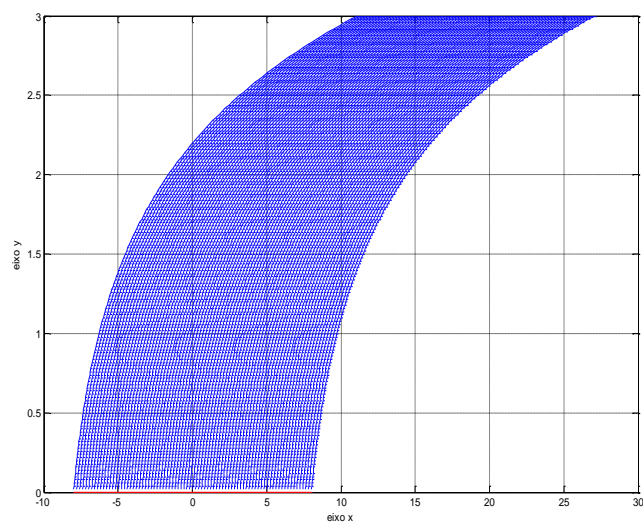

(b) Projeção das curvas características

Figura 1.22: Solução da equação $e^{y} u_{x}+u_{y}=c u, \operatorname{com} c=0$ e condição inicial $u(x, 0)=\cos x$ 


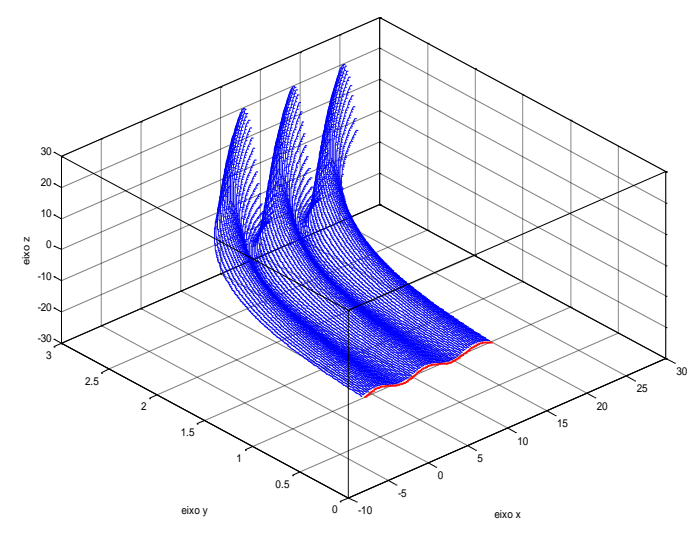

(a) Curvas características e Curva inicial

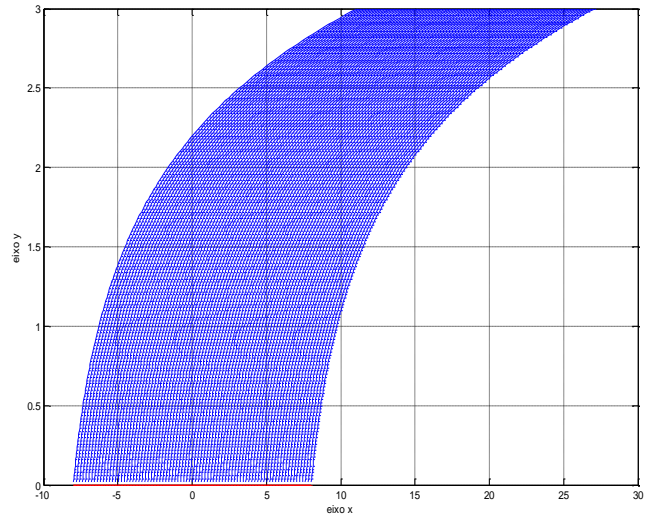

(b) Projeção das curvas características

Figura 1.23: Solução da equação $e^{y} u_{x}+u_{y}=c u, \operatorname{com} c=1$ e condição inicial $u(x, 0)=\cos x$

\subsection{Solução de EDPs Não Lineares pelo método das ca- racterísticas}

Um caso especial de equações não lineares são as equações quase-lineares que tem a forma

$$
R_{1}(x, y, u) u_{x}+R_{2}(x, y, u) u_{y}=T(x, y, u)
$$

para a solução das EDPs quase-lineares é possível usar o método das características impondo condições similares as equações lineares, podemos obter sua solução mediante um sistema dado por

$$
\left\{\begin{aligned}
\frac{d X}{d s} & =R_{1} \\
\frac{d Y}{d s} & =R_{2} \\
\frac{d U}{d s} & =T
\end{aligned}\right.
$$

Exemplo 1.12 Seja a equação

$$
\begin{gathered}
u u_{x}+u_{y}=0 \\
u(x, 0)=f(x)
\end{gathered}
$$

Sendo $f$ diferenciável, ao parametrizar a curva inicial por

$$
x=t, y=0, u=f(t)
$$

temos que as equações características estão dadas por 


$$
\left\{\begin{aligned}
\frac{d X}{d s}=U & & X(0)=t \\
\frac{d Y}{d s}=1 & & Y(0)=0 \\
\frac{d U}{d s}=0 & & U(0)=f(t)
\end{aligned}\right.
$$

daqui $u=$ constante, então

$$
U(s, t)=U(0, t)=f(t)
$$

por outro lado sendo $\frac{d X}{d s}=U=f(t)$ obtemos

$$
X(s, t)=s f(t)+t
$$

a sua vez como $\frac{d Y}{d s}=1$, obtemos que $Y(s, t)=s$, e portanto

$$
u=f(x-y u)
$$

Logo, considerando o caso particular $u(x, 0)=f(x)=-x$, e substituindo na solução encontrada temos $u=-(x-y u)$ obtemos $u=\frac{x}{y-1}$, finalmente o gráfico desta solução é dado pela Figura 1.24(a), 1.24(b)

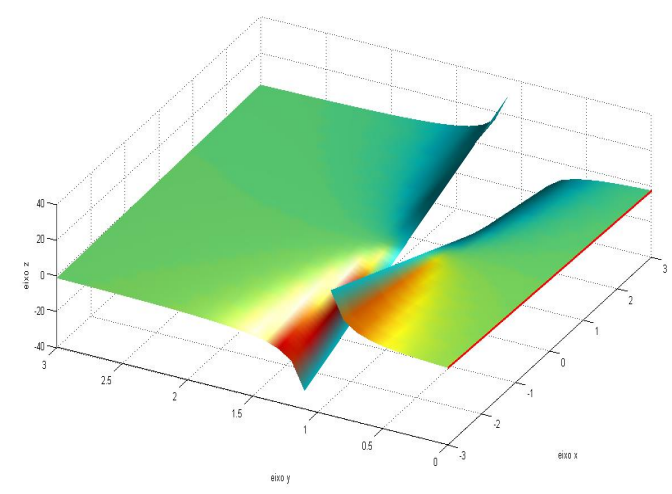

(a) Solução para $u(x, 0)=-x$

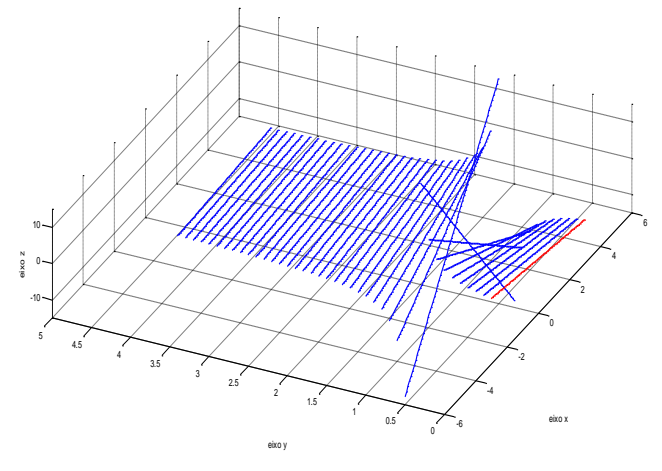

(b) Curvas características para $u(x, 0)=-x$

Figura 1.24: Solução da equação $\quad u u_{x}+u_{y}=0$ com condição inicial $u(x, 0)=-x$

Notamos que as curvas características giram em torno do origem, e que o movimento da onda não esta definido em $y=1$.

No caso particular onde $u(x, 0)=f(x)=x$, ao substituir na solução encontrada, temos $u=x-y u, \log 0 u=\frac{x}{y+1}$, notamos que o movimento da onda não está definido em $y=-1$, assim ao considerar o caso particular, onde $y>-1$ temos que o movimento de onda está definido, isto está ilustrado no gráfico dado pela Figura 1.25(a), 1.25(b). 


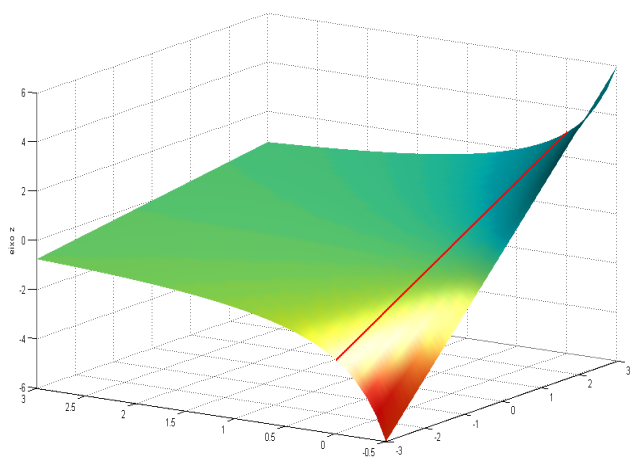

(a) Solução para $u(x, 0)=x$

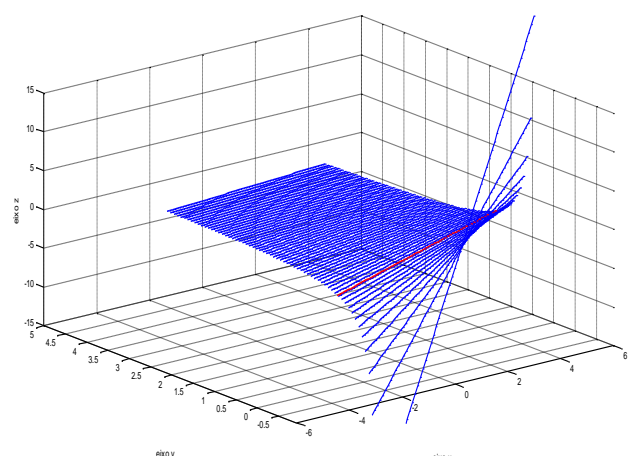

(b) Curvas características para $u(x, 0)=x$

Figura 1.25: Solução da equação $u u_{x}+u_{y}=0$ com condição inicial $u(x, 0)=x$

Uma EDP quase-linear de primeira ordem pode ser expressa como segue

$$
\left(R_{1}, R_{2}, T\right) \cdot\left(u_{x}, u_{y},-1\right)=0
$$

Dependendo do tipo de representação que se utilize para descrever a superfície propomos duas diferentes interpretações:

No caso que a representação seja explícita $u=f(x, y)$ e sendo que $p=u_{x}, q=u_{y}$, o vetor $N=(p, q,-1)$ representará o vetor normal à superfície, também poderíamos associar o campo vetorial $G: \Omega \rightarrow \mathbf{R}^{3}$, onde $\Omega \subset \mathbf{R}^{3}$ definido por

$$
G(x, y, z)=\left(R_{1}(x, y, u), R_{2}(x, y, u), T(x, y, u)\right)
$$

que verifica $N \cdot G=0$, indicando que o campo vetorial $G$ é perpendicular ao vetor normal de $u$ em cada ponto.

Por outro lado se a superfície determina-se implicitamente pela equação $S(x, y, z)=0$, seu vetor normal dado por $N=\left(S_{x}, S_{y}, S_{z}\right)$ deve verificar

$$
\left(R_{1}, R_{2}, T\right) \cdot\left(S_{x}, S_{y}, S_{z}\right)=0
$$

Para uma curva inicial parametrizada $(X(t), Y(t), U(t))$, com vetor tangente $\left(X^{\prime}(t), Y^{\prime}(t), U^{\prime}(t)\right)$, deverá existir um campo vetorial $G=\left(G_{1}, G_{2}, G_{3}\right)$ que contenha os vetores tangentes da curva inicial, tal que o vetor tangente seja perpendicular ao vetor normal da superfície

Analisaremos agora o caso de uma EDP implícita de primeira ordem no caso geral. Para isso consideraremos uma equação diferencial parcial implícita de duas variáveis

$$
F(x, y, u, p, q)=0
$$


onde $p=u_{x}, q=u_{y}$, cuja superfície solução contém uma curva característica inicial dada por $(X(t), Y(t), U(t))$, sendo $U(t)=u(X(t), Y(t))$ com vetores normais $N=(P(t), Q(t),-1)$ tal que $p=P(t), q=Q(t)$, temos que os vetores

$$
(p, q,-1) \quad \text { e } \quad\left(F_{p}, F_{q}, p F_{p}+q F_{q}\right)
$$

são perpendiculares, mais ainda utilizando a definição de curva característica (Jonh, 1982; Rhee e Amundson, 1986; Debnath, 2012) é possível estabelecer o seguinte

$$
\left\{\begin{aligned}
\frac{d X}{d t} & =F_{p} \\
\frac{d Y}{d t} & =F_{q} \\
\frac{d U}{d t} & =p F_{p}+q F_{q}
\end{aligned}\right.
$$

também derivando parcialmente a equação (1.1) em relação a suas variáveis obtemos

$$
\begin{gathered}
\frac{\partial F}{\partial x} \frac{\partial x}{\partial x}+\frac{\partial F}{\partial y} \frac{\partial y}{\partial x}+\frac{\partial F}{\partial u} \frac{\partial u}{\partial x}+\frac{\partial F}{\partial p} \frac{\partial p}{\partial x}+\frac{\partial F}{\partial q} \frac{\partial q}{\partial x}=0 \\
F_{x}+F_{u} p+F_{p} \frac{\partial p}{\partial x}+F_{q} \frac{\partial q}{\partial x}=0 \\
\frac{\partial F}{\partial y} \frac{\partial x}{\partial y}+\frac{\partial F}{\partial y} \frac{\partial y}{\partial y}+\frac{\partial F}{\partial u} \frac{\partial u}{\partial y}+\frac{\partial F}{\partial p} \frac{\partial p}{\partial y}+\frac{\partial F}{\partial q} \frac{\partial q}{\partial y}=0 \\
F_{y}+F_{u} q+F_{p} \frac{\partial p}{\partial y}+F_{q} \frac{\partial q}{\partial y}=0
\end{gathered}
$$

das equações (1.19) e (1.20) obtemos

$$
\begin{aligned}
& F_{p} \frac{\partial p}{\partial x}+F_{q} \frac{\partial q}{\partial x}=-F_{x}-F_{u} p \\
& F_{p} \frac{\partial p}{\partial y}+F_{q} \frac{\partial q}{\partial y}=-F_{y}-F_{u} q
\end{aligned}
$$

por outro lado usando a regra da cadeia

$$
\left\{\begin{array}{l}
\frac{d P}{d t}=\frac{\partial p}{\partial X} \frac{\partial X}{\partial t}+\frac{\partial p}{\partial Y} \frac{\partial Y}{\partial t}=\frac{\partial p}{\partial x} F_{p}+\frac{\partial p}{\partial y} F_{q} \\
\frac{d Q}{d t}=\frac{\partial q}{\partial X} \frac{\partial X}{\partial t}+\frac{\partial q}{\partial Y} \frac{\partial Y}{\partial t}=\frac{\partial q}{\partial x} F_{p}+\frac{\partial q}{\partial y} F_{q}
\end{array}\right.
$$

assim devido ao teorema de Schwartz

$$
\frac{\partial q}{\partial x}=\frac{\partial}{\partial x}\left(\frac{\partial u}{\partial y}\right)=\frac{\partial}{\partial y}\left(\frac{\partial u}{\partial x}\right)=\frac{\partial p}{\partial y}
$$


da equação (1.21) deduzimos que

$$
\left\{\begin{array}{l}
\frac{d P}{d t}=-F_{x}-p F_{u} \\
\frac{d Q}{d t}=-F_{y}-q F_{u}
\end{array}\right.
$$

portanto obtemos o sistema

$$
\left\{\begin{array}{l}
\frac{d X}{d t}=F_{p} \\
\frac{d Y}{d t}=F_{q} \\
\frac{d U}{d t}=p F_{p}+q F_{q} \\
\frac{d P}{d t}=-F_{x}-p F_{u} \\
\frac{d Q}{d t}=-F_{y}-q F_{u}
\end{array}\right.
$$

este sistema obtido será denominado "sistema característico".

Por outro lado, se para a EDP temos uma curva inicial a qual esta dada em forma paramétrica por $(X(t), Y(t), U(t), P(t), Q(t))$, esta curva deve verificar a equação diferencial e usando a regra da cadeia podemos estabelecer o seguinte

$$
\begin{aligned}
& \text { i) } \quad F(X(t), Y(t), U(t), P(t), Q(t))=0 \\
& \text { ii) } \quad \frac{d U}{d t}=P(t) X^{\prime}(t)+Q(t) Y^{\prime}(t)
\end{aligned}
$$

assim para achar uma curva característica usando uma curva inicial estaria descrito por

$$
\left\{\begin{array}{lrl}
\frac{d X}{d s}=F_{p} & X(0)=X(t) \\
\frac{d Y}{d s}=F_{q} & Y(0)=Y(t) \\
\frac{d U}{d s}=p F_{p}+q F_{q} & & U(0)=U(t) \\
\frac{d P}{d s}=-F_{x}-p F_{u} & P(0)=P(t) \\
\frac{d Q}{d s}=-F_{y}-q F_{u} & Q(0)=Q(t)
\end{array}\right.
$$

onde $X(0, t)=X(t), Y(0, t)=Y(t), U(0, t)=U(t), P(0, t)=P(t), Q(0, t)=Q(t)$ verificam as condições (1.24)

Agora resolveremos inicialmente a equação do transporte, para comparar que as soluções já obtidas são iguais usando este método, depois resolveremos os casos de equações não lineares 
Exemplo 1.13 Seja a equação

$$
\begin{gathered}
a u_{x}+u_{y}=0 \\
u(x, 0)=f(x) \\
F\left(x, y, u, u_{x}, u_{y}\right)=a u_{x}+u_{y}=0
\end{gathered}
$$

como $p=u_{x}, q=u_{y}$ a equação descreve-se como

$$
F\left(x, y, u, u_{x}, u_{y}\right)=a p+q=0
$$

$\log 0$

$$
\left\{\begin{array}{l}
\frac{d X}{d s}=a \\
\frac{d Y}{d s}=1 \\
\frac{d U}{d s}=a p+q \\
\frac{d P}{d s}=0 \\
\frac{d Q}{d s}=0
\end{array}\right.
$$

parametrizando a curva inicial temos $(t, 0, f(t))$, logo

$$
\left\{\begin{array}{llrl}
\frac{d X}{d s} & =a & X(0) & =t \\
\frac{d Y}{d s} & =1 & Y(0) & =0 \\
\frac{d U}{d s} & =a P+Q & & U(0)=f(t)
\end{array}\right.
$$

neste caso particular poderíamos integrar diretamente pois $P(s, t)=P(s) \equiv$ constante, $Q(s, t)=Q(s) \equiv$ constante e mais ainda sendo $\frac{d U}{d s}=0$ pela forma da equação concluímos que $U(s, t)=U(s) \equiv$ constante

Pelas condições $(i),(i i)$ obtemos

$$
\begin{gathered}
a P(t)+Q(t)=0 \\
f^{\prime}(t)=P(t) \cdot 1+Q(t) \cdot 0
\end{gathered}
$$

$\operatorname{assim} P(t)=f^{\prime}(t), Q(t)=-a f^{\prime}(t)$

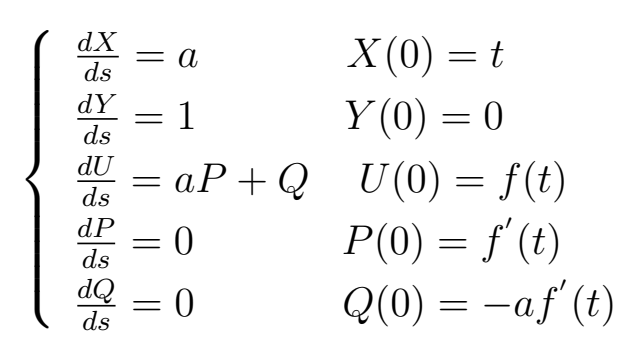

daqui 
- $X(s, t)=a s+t$

- $Y(s, t)=s$

- $U(s, t)=f(t)$

Portanto, sendo $U(s, t)=f(X(s, t)-a Y(s, t))$ e como $U(s, t)=u(X(s, t), Y(s, t)))$, a solução é $u(x, y)=f(x-a y)$. Para o caso particular em que $a=0.5$ e $f(x)=e^{-x^{2}}$ temos que

$$
\begin{aligned}
& 0.5 u_{x}+u_{y}=0 \\
& u(x, 0)=e^{-x^{2}}
\end{aligned}
$$

tem solução $u(x, y)=e^{-(x-0.5 y)^{2}}$

Podemos visualizar a solução descrita nas Figuras 1.26(a), 1.26(b)

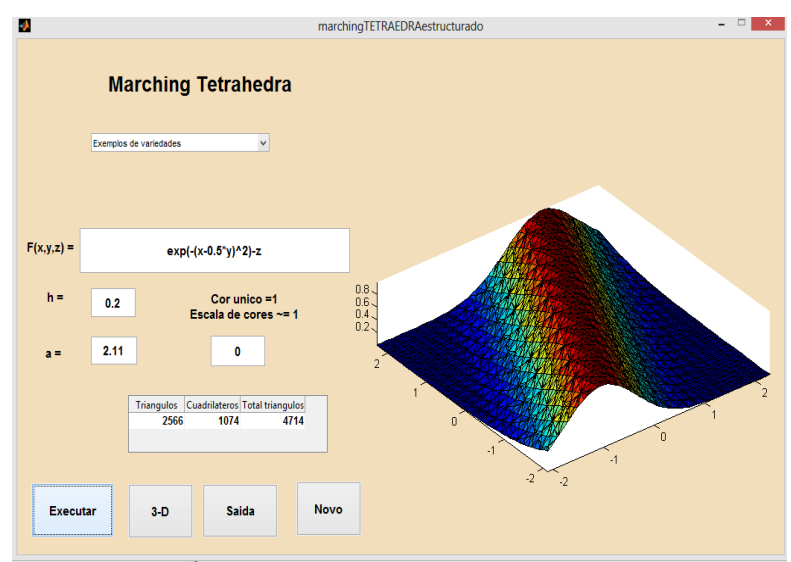

(a) Interface Marching tetrahedra

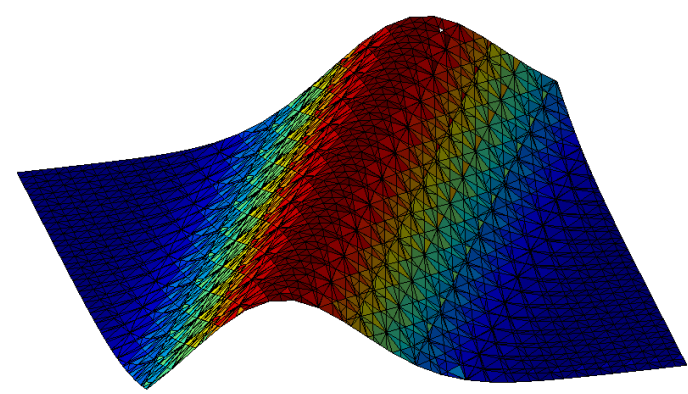

(b) $u(x, y)=e^{-(x-0.5 y)^{2}}$

Figura 1.26: Visualização da solução $u(x, y)=e^{-(x-0.5 y)^{2}}$ usando Marching tetrahedra

Exemplo 1.14 Seja a equação

$$
\begin{gathered}
\left(u_{x}\right)^{2}+\left(u_{y}\right)^{2}=1 \\
x^{2}+y^{2}=1, u=0 \\
F\left(x, y, u, u_{x}, u_{y}\right)=\left(u_{x}\right)^{2}+\left(u_{y}\right)^{2}-1=0
\end{gathered}
$$

como $p=u_{x}, q=u_{y}$ a equação descreve-se como

$$
F\left(x, y, u, u_{x}, u_{y}\right)=p^{2}+q^{2}-1=0
$$


$\log 0$

$$
\left\{\begin{array}{l}
\frac{d X}{d s}=2 p \\
\frac{d Y}{d s}=2 q \\
\frac{d U}{d s}=2 p^{2}+2 q^{2} \\
\frac{d P}{d s}=0 \\
\frac{d Q}{d s}=0
\end{array}\right.
$$

parametrizando a curva inicial temos $(X(t), Y(t), U(t))=(\cos (t), \sin (t), 0)$ na projeção do espaço $\mathbf{R}^{3}, \operatorname{logo}$

$$
\left\{\begin{array}{lrl}
\frac{d X}{d s} & =2 P & X(0)=\cos (t) \\
\frac{d Y}{d s}=2 Q & Y(0)=\sin (t) \\
\frac{d U}{d s}=2 P^{2}+2 Q^{2} & U(0)=0
\end{array}\right.
$$

Pelas condições $(i),(i i)$ obtemos

$$
\begin{gathered}
P(t)^{2}+Q(t)^{2}=1 \\
0=-P(t) \cdot \sin (t)+Q(t) \cdot \cos (t)
\end{gathered}
$$

obtemos duas possibilidades na solução do sistema não linear

a) $P(t)=\cos (t), Q(t)=\sin (t)$

b) $P(t)=-\cos (t), Q(t)=-\sin (t)$

tomando a possibilidade $(a)$

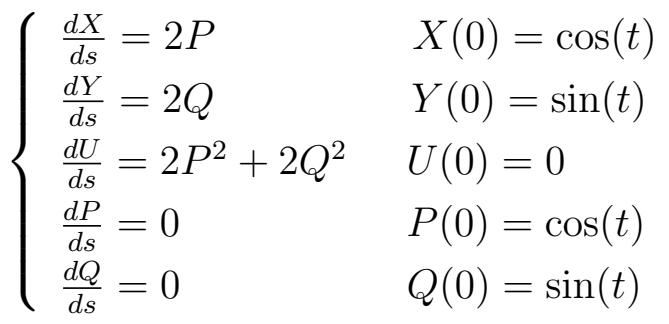

daqui

- $X(s, t)=(2 s+1) \cos (t)$

- $Y(s, t)=(2 s+1) \sin (t)$

- $U(s, t)=2 s$ 


$$
X(s, t)^{2}+Y(s, t)^{2}=(U(s, t)+1)^{2}
$$

Portanto, a solução é $x^{2}+y^{2}=(u+1)^{2}$. Analogamente para a possibilidade $(b)$ a solução estaria dada por $x^{2}+y^{2}=(1-u)^{2}$

Podemos visualizar a solução descrita nas Figuras 1.27(a), 1.27(b)

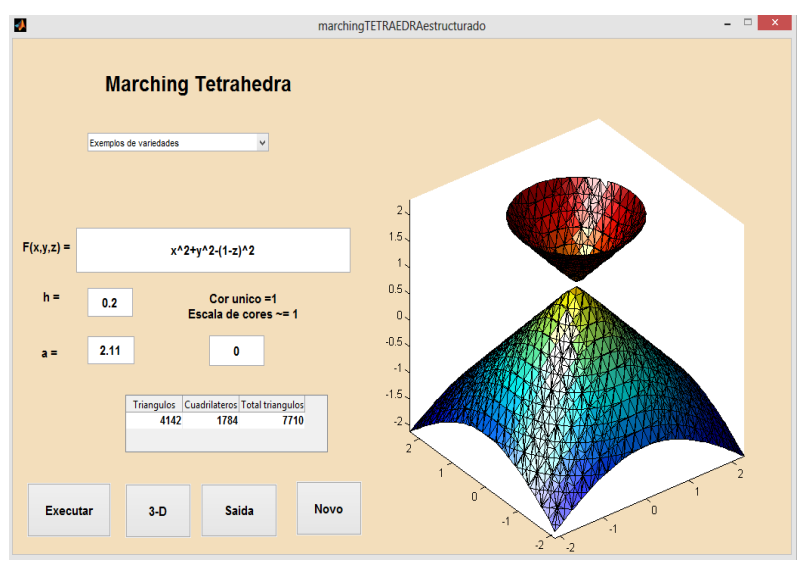

(a) Interface Marching tetrahedra

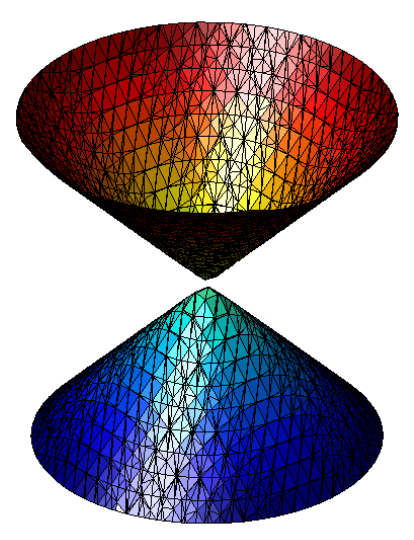

(b) $x^{2}+y^{2}=(1-u)^{2}$

Figura 1.27: Visualização da solução $x^{2}+y^{2}=(1-u)^{2}$ usando Marching tetrahedra

Exemplo 1.15 Seja a equação

$$
\begin{gathered}
\left(u_{x}\right)^{2}+u_{y}+u=0 \\
u(x, 0)=x \\
F\left(x, y, u, u_{x}, u_{y}\right)=\left(u_{x}\right)^{2}+u_{y}+u=0
\end{gathered}
$$

como $p=u_{x}, q=u_{y}$ a equação descreve-se como

$$
F\left(x, y, u, u_{x}, u_{y}\right)=p^{2}+q+u=0
$$

$\log 0$

$$
\left\{\begin{array}{l}
\frac{d X}{d s}=2 p \\
\frac{d Y}{d s}=1 \\
\frac{d U}{d s}=2 p^{2}+q \\
\frac{d P}{d s}=-p \\
\frac{d Q}{d s}=-q
\end{array}\right.
$$

parametrizando a curva inicial temos $(X(t), Y(t), U(t))=(t, 0, t)$ na projeção do espaço $\mathbf{R}^{3}$, logo 


$$
\left\{\begin{array}{lrl}
\frac{d X}{d s}=2 P & X(0)=t \\
\frac{d Y}{d s}=1 & Y(0)=0 \\
\frac{d U}{d s}=2 P^{2}+Q & U(0)=t
\end{array}\right.
$$

Pelas condições $(i),(i i)$ obtemos

$$
\begin{gathered}
P(t)^{2}+Q(t)+U(t)=0 \\
1=P(t) \cdot 1+Q(t) \cdot 0
\end{gathered}
$$

obtemos

$$
\begin{aligned}
& P(t)=1 \\
& Q(t)=-(1+t)
\end{aligned}
$$

$$
\begin{cases}\frac{d X}{d s}=2 P & X(0)=t \\ \frac{d Y}{d s}=1 & Y(0)=0 \\ \frac{d U}{d s}=2 P^{2}+Q & U(0)=t \\ \frac{d P}{d s}=-P & P(0)=1 \\ \frac{d Q}{d s}=-Q & Q(0)=-(1+t)\end{cases}
$$

daqui

- $P(s, t)=e^{-s}$

- $Q(s, t)=-(1+t) e^{-s}$

- $X(s, t)=-2 s e^{-s}+t+2$

- $Y(s, t)=s$

- $U(s, t)=-e^{-2 s}+(1+t) e^{-s}$

Portanto, a solução é $u(x, y)=e^{2 y}+(x-1) e^{-y}$.

Podemos visualizar a solução descrita nas Figuras 1.28(a), 1.28(b) 


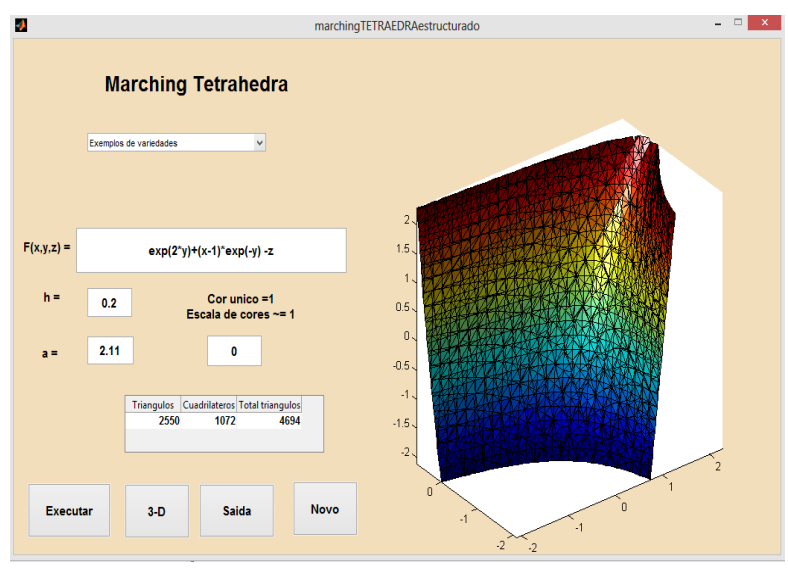

(a) Interface Marching tetrahedra

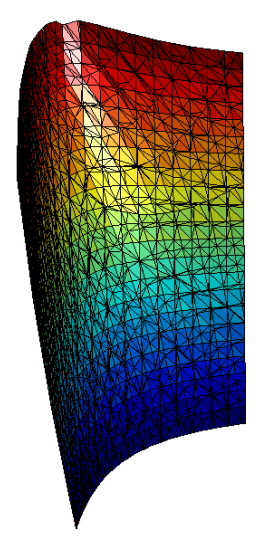

(b) $u(x, y)=e^{2 y}+(x-1) e^{-y}$

Figura 1.28: Visualização da solução $u(x, y)=e^{2 y}+(x-1) e^{-y}$ usando Marching tetrahedra 


\section{CAPÍTULO}

\section{2}

\section{Existência e unicidade da solução}

Enunciaremos neste capítulo o teorema de Cauchy que estabelece as condições para garantir a existência e unicidade da solução de uma EDP implícita de primeira ordem.

Para estabelecer o Teorema generalizado usaremos a notação determinada como na equação (1.4), assim uma equação diferencial parcial implícita em duas variáveis é dada por

$$
F\left(x_{1}, x_{2}, u, p_{1}, p_{2}\right)=0
$$

onde $p_{1}=u_{x_{1}}, p_{2}=u_{x_{2}}$, cuja superfície solução contém uma curva característica inicial dada por $\left(X_{1}(t), X_{2}(t), U(t)\right)$, sendo $U(t)=u\left(X_{1}(t), X_{2}(t)\right)$, com vetores normais $N=\left(P_{1}(t), P_{2}(t),-1\right)$ tal que $p_{1}=P_{1}(t), p_{2}=P_{2}(t)$, os vetores

$$
\left(p_{1}, p_{2},-1\right) \quad \text { e } \quad\left(F_{p_{1}}, F_{p_{2}}, p_{1} F_{p_{1}}+p_{2} F_{p_{2}}\right)
$$

são perpendiculares, e o sistema característico está dado por

$$
\left\{\begin{array}{l}
\frac{d X_{1}}{d t}=F_{p_{1}} \\
\frac{d X_{2}}{d t}=F_{p_{2}} \\
\frac{d U}{d t}=p_{1} F_{p_{1}}+p_{2} F_{p_{2}} \\
\frac{d P_{1}}{d t}=-F_{x_{1}}-p_{1} F_{u} \\
\frac{d P_{2}}{d t}=-F_{x_{2}}-p_{2} F_{u}
\end{array}\right.
$$


Para determinar as soluções de um sistema característico, assumimos que

$$
U(s, t)=u(X(s, t), Y(s, t))
$$

assim conseguimos encontrar a solução, no entanto isso só é possível se verifica-se o teorema da função inversa.

Teorema da Função Inversa 2.1 Assumindo que $G: M \subset \mathbf{R}^{n} \rightarrow \mathbf{R}^{n}$ é uma função de classe $\mathbf{C}^{1}$ e que $J G\left(x_{0}\right) \neq 0$ onde $J\left(G\left(x_{0}\right)\right)$ é o Jacobiano de $G$ no ponto $x_{0}$. Então existe um conjunto aberto $V \subset M$ com $x_{0} \in V$ e um aberto $W \subset \mathbf{R}^{n}$, com $G\left(x_{0}\right) \in W$ tal que

$$
G: V \rightarrow W
$$

é bijetora, e a função inversa

$$
G^{-1}: W \rightarrow V
$$

é de classe $\mathbf{C}^{1}$.

\subsection{Teorema De Cauchy}

Teorema 2.2 Dada a equação diferencial implícita

$$
F\left(x_{1}, x_{2}, u, u_{x_{1}}, u_{x_{2}}\right)=0
$$

onde $F \in \mathbf{C}^{2}(\Omega), \Omega \subset \mathbf{R}^{2} \times \mathbf{R} \times \mathbf{R}^{2} e\left|F_{p_{1}}\right|+\left|F_{p_{2}}\right|>0$, sejam $X_{1}^{0}(t), X_{2}^{0}(t)$ e $U^{0}(t)$ definidas em $I \subset \mathbf{R}$ com segunda derivada continua em $I$. Se $P_{1}^{0}(t), P_{2}^{0}(t)$ tem primeira derivada continua em I e satisfazem as seguintes condições

(i) Condições de banda

$$
\left\{\begin{array}{c}
\frac{d U^{0}}{d t}(t)=\frac{d X_{1}^{0}}{d t} P_{1}^{0}(t)+\frac{d X_{2}^{0}}{d t} P_{2}^{0}(t) \\
F\left(X_{1}^{0}(t), X_{2}^{0}(t), U^{0}(t), P_{1}^{0}(t), P_{2}^{0}(t)\right)=0
\end{array}\right.
$$

(ii) Condições de transversalidade

$$
\operatorname{det}\left[\begin{array}{cc}
\frac{d X_{1}^{0}}{d t} & \frac{d X_{2}^{0}}{d t} \\
F_{p_{1}}\left(X_{1}^{0}(t), X_{2}^{0}(t), U^{0}(t), P_{1}^{0}(t), P_{2}^{0}(t)\right) & F_{p_{2}}\left(X_{1}^{0}(t), X_{2}^{0}, U^{0}(t), P_{1}^{0}(t), P_{2}^{0}(t)\right)
\end{array}\right] \neq 0
$$

então existe uma única função $\phi$ definida por $\phi: V \rightarrow \mathbf{R}, V \subset \mathbf{R}^{2}$ que verifica

$$
\left\{\begin{array}{c}
F\left(x_{1}, x_{2}, \phi\left(x_{1}, x_{2}\right), \phi_{x_{1}}\left(x_{1}, x_{2}\right), \phi_{x_{2}}\left(x_{1}, x_{2}\right)\right)=0, \forall\left(x_{1}, x_{2}\right) \in V \\
\phi\left(X_{1}^{0}(t), X_{2}^{0}(t)\right)=U^{0}(t) \\
\phi_{x_{1}}\left(X_{1}^{0}(t), X_{2}^{0}(t)\right)=P_{1}^{0}(t) \\
\phi_{x_{2}}\left(X_{1}^{0}(t), X_{2}^{0}(t)\right)=P_{2}^{0}(t)
\end{array}\right.
$$

assim ф é a solução única da equação diferencial implícita. 
Demostração: A prova deste teorema pode-se encontrar em (Ireneo, 1995).

\subsection{Teorema de Cauchy generalizado}

Uma Equação diferencial parcial implícita de primeira ordem em $n$ variáveis, com $n \geq 3$ pode ser denotada como

$$
F\left(x_{1}, \ldots, x_{n}, u, u_{x_{1}}, \ldots, u_{x_{n}}\right)=0
$$

ou equivalentemente

$$
F\left(x_{1}, \ldots, x_{n}, u, p_{1}, \ldots, p_{n}\right)=0
$$

onde os $p_{i}$ denotam as derivadas parciais $p_{i}=\frac{\partial u}{\partial x_{i}}$, para simplificar a notação podemos denotar $x=\left(x_{1}, x_{2}, \ldots, x_{n}\right)$ e $\nabla u=\left(u_{x_{1}}, u_{x_{2}}, \ldots, u_{x_{n}}\right)$ ou equivalentemente $p=\left(p_{1}, p_{2}, \ldots, p_{n}\right)$, assim a equação descreve-se como

$$
F(x, u, \nabla u)=0
$$

onde $F \in C^{2}$ verifica

$$
\begin{gathered}
F: \Omega \subset \mathbf{R}^{n} \times \mathbf{R} \times \mathbf{R}^{n} \rightarrow \mathbf{R} \\
\left\|\nabla_{p} F\right\| \equiv\left\|\left(F_{p_{1}}, \ldots, F_{p_{n}}\right)\right\|>0
\end{gathered}
$$

num aberto $\Omega$, com $\Omega \subset \mathbf{R}^{2 n+1}$, supondo que $u: G \subset \mathbf{R}^{n} \rightarrow \mathbf{R}$ é solução de (2.4).

Analogamente ao teorema anterior, consideramos no espaço (n-1)-dimensional como dado inicial, uma superfície inicial dada em forma paramétrica por

$$
\begin{aligned}
x_{i} & =X_{i}\left(t_{1}, \ldots, t_{n-1}\right) \\
u & =U\left(t_{1}, \ldots, t_{n-1}\right)
\end{aligned}
$$

assim o sistema de equações diferenciais característico é generalizado como:

$$
\left\{\begin{array}{l}
\frac{d x_{i}}{d s}=F_{p_{i}} \\
\frac{d u}{d s}=\sum_{i=1}^{n} p_{i} F_{p_{i}} \\
\frac{p_{i}}{d s}=-F_{x_{i}}-p_{i} F_{u}
\end{array}\right.
$$

derivando a equação (2.3) obtemos

$$
\frac{d F}{d s}=\sum_{i=1}^{n} F_{x_{i}} \frac{d x_{i}}{d s}+F_{u} \frac{d u}{d s}+\sum_{i=1}^{n} F_{p_{i}} \frac{d p_{i}}{d s}=0
$$


logo para achar a solução paramétrica temos as condições iniciais

$$
\begin{aligned}
& x_{i}\left(t_{1}, \ldots, t_{n-1}, 0\right)=X_{i}\left(t_{1}, \ldots, t_{n-1}\right) \\
& u\left(t_{1}, \ldots, t_{n-1}, 0\right)=U\left(t_{1}, \ldots, t_{n-1}\right)
\end{aligned}
$$

para achar as $n$ condições restantes temos que

$$
p_{i}\left(t_{1}, \ldots, t_{n-1}, 0\right)=P_{i}\left(t_{1}, \ldots, t_{n-1}\right)
$$

as condições iniciais (2.6) devem verificar a equação diferencial implícita (2.3) isto é

$$
F\left(X_{1}, \ldots, X_{n}, U, P_{1}, \ldots, P_{n}\right)=0
$$

além disso

$$
\frac{\partial U}{\partial t_{i}}=\sum_{j=1}^{n} P_{j} \frac{\partial X_{j}}{\partial t_{i}} \quad i=1, \ldots n-1
$$

para simplificar a notação denotaremos $x=\left(x_{1}, x_{2}, \ldots, x_{n}\right)$ e $\nabla u=\left(u_{x_{1}}, u_{x_{2}}, \ldots, u_{x_{n}}\right)$ ou equivalentemente $p=\left(p_{1}, p_{2}, \ldots, p_{n}\right)$

Teorema 2.3 Seja $F$ tal que $F \in C^{2}(\Omega)$ com $\Omega \subset \mathbf{R}^{2 n+1}$ verifica as hipóteses descritas, considerando o problema de valor inicial

$$
\left\{\begin{array}{l}
F(x, u, \nabla u)=0 \\
u(\alpha(t))=\phi(t) \quad t \in U
\end{array}\right.
$$

temos que sendo

$$
\alpha: U \subset \mathbf{R}^{n-1} \rightarrow \mathbf{R} \quad \text { e } \phi: U \rightarrow \mathbf{R}
$$

funções com duas derivadas contínuas, tais que $\alpha\left(t^{0}\right)=x^{0}, \phi\left(t^{0}\right)=u^{0}, p^{0}$ os dados iniciais tais que,

1. $F\left(x^{0}, u^{0}, p^{0}\right)=0$

2. $p^{0} \alpha_{t}\left(t^{0}\right)=\nabla_{t} \phi\left(t^{0}\right)$

3. $\left.\operatorname{det}\left(\alpha_{t}\left(t^{0}\right)\right), \nabla_{p} F\left(x^{0}, u^{0}, p^{0}\right)\right) \neq 0$

Então existe $G$, vizinhança de $x^{0}$ em $\mathbf{R}^{n} e$

$$
u: G \rightarrow \mathbf{R}
$$

tal que

$$
\left\{\begin{array}{l}
F(x, u(x), \nabla u(x)=0 \quad \text { para } \quad x \in G \\
u(\alpha(t))=\phi(t) \quad t \in U \text { tal que } \alpha(t) \in G
\end{array}\right.
$$


Demostração: A prova deste teorema pode-se encontrar em (Ireneo, 1995).

\section{Exemplo 2.1 Seja}

$$
\begin{gathered}
u_{x_{1}}+u_{x_{2}}+u_{x_{3}}+u=0 \\
u\left(x_{1}, x_{2}, 0\right)=f\left(x_{1}, x_{2}\right)
\end{gathered}
$$

então

$$
F\left(x_{1}, x_{2}, x_{3}, u, u_{x_{1}}, u_{x_{2}}, u_{x_{3}}\right)=u_{x_{1}}+u_{x_{2}}+u_{x_{3}}+u=0
$$

parametrizando a superfície inicial temos

$$
\alpha\left(t_{1}, t_{2}\right)=\left(X_{1}\left(t_{1}, t_{2}\right), X_{2}\left(t_{1}, t_{2}\right), X_{3}\left(t_{1}, t_{2}\right), U\left(t_{1}, t_{2}\right)\right)=\left(t_{1}, t_{2}, 0, f\left(t_{1}, t_{2}\right)\right)
$$

usando o sistema característico

$$
\left\{\begin{array}{l}
\frac{d X_{1}}{d s}=F_{P_{1}} \\
\frac{d X_{2}}{d s}=F_{P_{2}} \\
\frac{d X_{3}}{d s}=F_{P_{3}} \\
\frac{d U}{d s}=P_{1} F_{P_{1}}+P_{2} F_{P_{2}}+P_{3} F_{P_{3}} \\
\frac{d P_{1}}{d s}=-F_{X_{1}}-P_{1} F_{U} \\
\frac{d P_{2}}{d s}=-F_{X_{2}}-P_{2} F_{U} \\
\frac{d P_{3}}{d s}=-F_{X_{3}}-P_{3} F_{U}
\end{array}\right.
$$

e as condições

i) $\quad F\left(X_{1}\left(t_{1}, t_{2}\right), X_{2}\left(t_{1}, t_{2}\right), X_{3}\left(t_{1}, t_{2}\right), U\left(t_{1}, t_{2}\right), P_{1}\left(t_{1}, t_{2}\right), P_{2}\left(t_{1}, t_{2}\right), P_{3}\left(t_{1}, t_{2}\right)\right)=0$

ii) $\quad \frac{\partial U}{\partial t_{1}}=P_{1} \frac{\partial X_{1}}{\partial t_{1}}+P_{2} \frac{\partial X_{2}}{\partial t_{1}}+P_{3} \frac{\partial X_{3}}{\partial t_{1}}$

$\frac{\partial U}{\partial t_{2}}=P_{1} \frac{\partial X_{1}}{\partial t_{2}}+P_{2} \frac{\partial X_{2}}{\partial t_{2}}+P_{3} \frac{\partial X_{3}}{\partial t_{2}}$

sendo as condições iniciais dadas por

$$
X_{1}\left(t_{1}, t_{2}, 0\right)=t_{1}, X_{2}\left(t_{1}, t_{2}, 0\right)=t_{2}, X_{3}\left(t_{1}, t_{2}, 0\right)=0, U\left(t_{1}, t_{2}, 0\right)=f\left(t_{1}, t_{2}\right)
$$

obtemos o sistema característico

$$
\begin{cases}\frac{d X_{1}}{d s}=1 & X_{1}\left(t_{1}, t_{2}\right)=t_{1} \\ \frac{d X_{2}}{d s}=1 & X_{2}\left(t_{1}, t_{2}\right)=t_{2} \\ \frac{d X_{3}}{d s}=0 & X_{3}\left(t_{1}, t_{2}\right)=0 \\ \frac{d U}{d s}=-U & U\left(t_{1}, t_{2}\right)=f\left(t_{1}, t_{2}\right) \\ \frac{d P_{1}}{d s}=-P_{1} & P_{1}\left(t_{1}, t_{2}\right)=f_{t_{1}} \\ \frac{d P_{2}}{d s}=-P_{2} & P_{2}\left(t_{1}, t_{2}\right)=f_{t_{2}} \\ \frac{d P_{3}}{d s}=-P_{3} & P_{3}\left(t_{1}, t_{2}\right)=-U-f_{t_{1}}-f_{t_{2}}\end{cases}
$$


resolvendo o sistema característico obtemos

$$
X_{1}\left(t_{1}, t_{2}, s\right)=t_{1}+s, X_{2}\left(t_{1}, t_{2}, s\right)=t_{2}+s, X_{3}\left(t_{1}, t_{2}, s\right)=s, U\left(t_{1}, t_{2}, s\right)=f\left(t_{1}, t_{2}\right) e^{-s}
$$

assim podemos estabelecer as relações

$$
t_{1}=x_{1}-x_{3}, t_{2}=x_{2}-x_{3}, s=x_{3}
$$

por tanto a solução fica como

$$
u\left(x_{1}, x_{2}, x_{3}\right)=f\left(x_{1}-x_{3}, x_{2}-x_{3}\right) e^{-x_{3}} .
$$


Os métodos numéricos são elaborados para proporcionar soluções aproximadas a diversos tipos de problemas, como por exemplo resolver um problema que não admite solução analítica. Porém, a importância da eficácia do método, por outro lado nem todos os problemas matemáticos podem ser resolvidos por um computador, pois se sua solução não existe, não faz sentido realizar nenhuma aproximação numérica. Se a solução não é única ao menos localmente, tem risco de obter uma "solução numérica" que não esteja próxima da solução do problema original, dai a necessidade de verificar as hipóteses do teorema de existência e unicidade. Temos também que considerar que se o problema é sensível a perturbações não se pode garantir que a solução seja confiável, lembremos a existência de problemas que envolvem solução de sistemas de EDOs, tais como o atrator de Lorenz, que resolvidos numericamente podem apresentar estas dificuldades.

Nos métodos numéricos, nas soluções dos problemas os algoritmos requerem um modelo distinto e adequado de acordo a natureza do problema. Os algoritmos diferem também, pelo esforço computacional, complexidade do código, número de operações desenvolvidas, assim como o erro cometido nas iterações. Devemos considerar que na criação de um algoritmo não é suficiente que um problema seja bem formulado, pois se temos um algoritmo que resolva o problema, devemos garantir a convergência. Poderíamos resumir que a análise deve considerar tópicos como consistência, precisão, estabilidade e convergência (Quarteroni e Saleri, 2000; Atkinson e Stewart, 2009).

As técnicas numéricas que utilizaremos para a solução de EDPs implícitas de primeira ordem, baseiam-se em métodos clássicos de solução de EDOs, diferenças finitas para aproximar as derivadas parciais e técnicas dos métodos de continuação (Allgower e Georg, 2003). 


\subsection{Solução Numérica de Equações Diferenciais Ordiná- rias}

O problema de Cauchy, também denominado problema de valor inicial, para equações diferencias ordinárias no caso escalar ou vetorial consiste em achar a função que satisfaz a EDO e uma condição inicial. No caso escalar se $I \subset \mathbf{R}$ contém o ponto $t_{0}$, devemos achar a função solução $X \in C^{1}(I)$ tal que

$$
\left\{\begin{array}{l}
X^{\prime}(t)=f(t, X) \quad t \in I \\
X\left(t_{0}\right)=X_{0}
\end{array}\right.
$$

Se a função $f$ é localmente contínua e satisfaz a condição de Liptchitz em $\left(t_{0}, X_{0}\right)$ com respeito a $X$, o problema de Cauchy tem solução numa vizinhança de $t_{0}$.

Quando a função $f(t, X)$ não depende explicitamente de $t$ a equação diferencial se denomina autônoma.

Analogamente no caso vetorial para uma equação diferencial $X^{\prime}(t)=f(t, X, \lambda)$ se $J \subset \mathbf{R}$, $U \subset \mathbf{R}^{n}$ e $\Lambda \subset \mathbf{R}^{m}$ são conjuntos abertos, e $f: J \times U \times \Lambda \rightarrow \mathbf{R}^{n}$ uma função contínua, para um valor fixo de $\lambda \in \Lambda$ uma solução desta equação é uma função diferenciável $\varphi: J_{0} \rightarrow U$ definida sobre um intervalo aberto $J_{0} \subset J$

$$
\frac{d \varphi}{d t}(t)=f(t, \varphi(t), \lambda)
$$

para todo $t \in J_{0}, t_{0} \in J, X_{0} \in U$ e $\lambda_{0} \in \Lambda$, o problema de valor inicial esta dado por

$$
\left\{\begin{array}{l}
X^{\prime}(t)=f\left(t, X, \lambda_{0}\right) \\
X\left(t_{0}\right)=X_{0}
\end{array}\right.
$$

se a função $f$ satisfaz a condição de Liptchitz localmente, existirá uma solução ao problema de valor inicial segundo o teorema da existência e unicidade provado em (Chicone, 2006; Gameiro e Mischaikow, 2013).

As técnicas numéricas para resolver EDOs podem-se classificar em métodos de um passo e de passo múltiplo. Os métodos denominados de um só passo são aqueles onde no cálculo de cada ponto se utiliza informação do último ponto, os métodos de Euler, Taylor e Runge-Kutta são métodos de um só passo (Butcher, 2008).

Definição 3.1 (Método de um passo) Um método numérico que aproxima a solução $X$ do problema (3.1) pela função $x$, denomina-se método de um passo se para todo $k \geq 0, x_{k+1}$ depende só de $x_{k}$. Qualquer outro casso é chamado método Multi-passo 
Assim temos por exemplo como método de um passo o método de Euler, que tem suas formulas recursivas dadas por

Método de Euler para frente (Forward Euler)

$$
x_{i+1}=x_{i}+h f_{i}
$$

\section{Método de Euler para trás (Backward Euler)}

$$
x_{i+1}=x_{i}+h f_{i+1}
$$

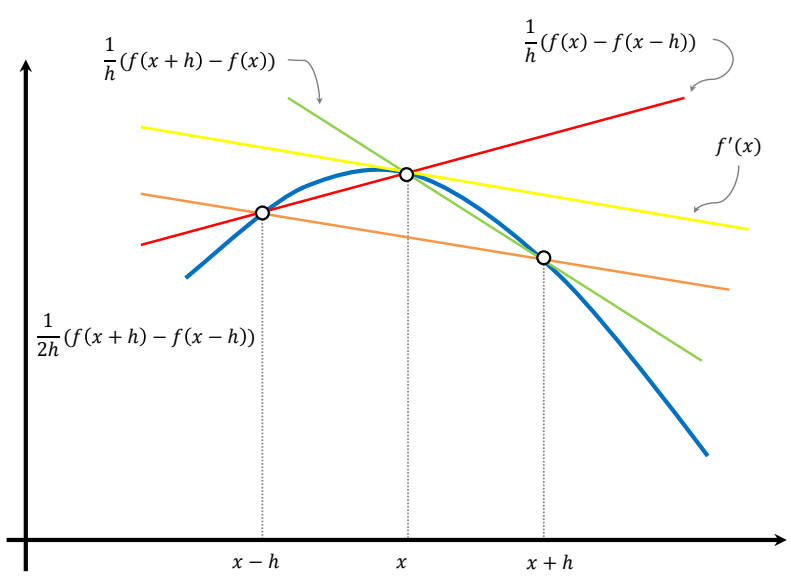

Figura 3.1: Aproximações da derivada

No método de Euler para trás cuja interpretação geométrica esta dada pela Figura 3.1, notemos que em seu equação iterativa

$$
x_{i+1}=x_{i}+h f_{i+1}
$$

existe uma dependência dos valores antigo e novo em ambos os lados porém esta fórmula também é denominada método implícito, assim para resolver uma EDO usando esta fórmula terá que resolver-se como equação não linear isto é

$$
g\left(x_{i+1}\right)=x_{i+1}-x_{i}-h f_{i+1}=0
$$

pudendo assim utilizar métodos para resolver equações não lineares tais como o método de Newton, no entanto o custo computacional será alto.

Se a solução estiver determinada no intervalo $\left[t_{0}, b\right]$ e este intervalo estivesse dividido em $n$ subintervalos o tamanho de passo é $h=\frac{b-t_{0}}{n}$ e $t_{k}=t_{0}+i h$, para $i=0,1, \ldots, n$, o objetivo é que o 
erro na discretização seja o mais baixo possível, porém o tamanho de passo $h$ deve-se aproximar a zero o que faz aumentar o número de nós da partição do intervalo $\left[t_{0}, b\right]$. Se a equação discretizada e o $P V I$ são equivalentes ao diminuir o tamanho de passo, o método é denominado consistente, ao denotar por $\tau_{i}$ ao erro de truncamento local e por $\tau$ ao erro de truncamento global sabemos que o método será consistente se

$$
\lim _{h \rightarrow 0} \tau=0
$$

a ordem de consistência é definido como o maior número natural $p$ tal que

$$
\tau \leq C h^{p}
$$

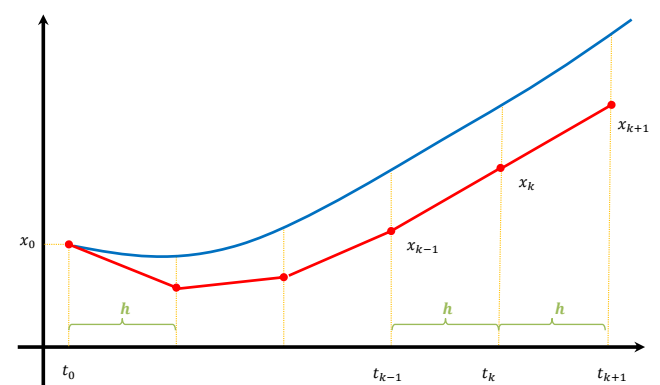

(a) Aproximação com passo constante

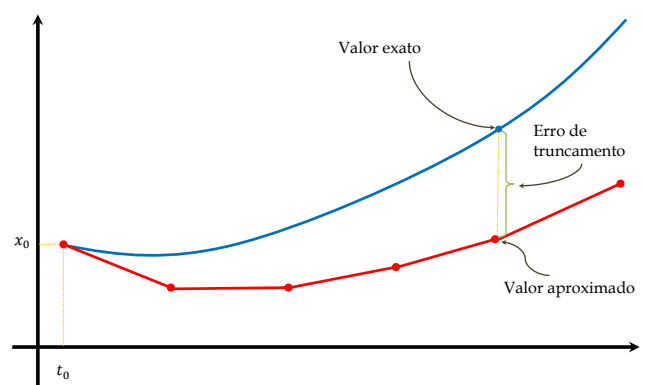

(b) Erro de truncamento

Figura 3.2: Passo constante e Erro de truncamento

No caso do método de Euler, usando o desenvolvimento de séries de Taylor concluímos que em cada passo da iteração

$$
\tau_{i}=\frac{h}{2} x^{\prime \prime}\left(\xi_{i}\right), \quad \xi_{i} \in\left[t_{i}, t_{i+1}\right]
$$

o que permite concluir que o método de Euler é um método consistente de ordem $O(h)$, assim para conseguir uma melhor aproximação tem-se que reduzir o tamanho de passo, o que ocasiona um maior cálculo do número de operações, uma maneira de reduzir o erro adequadamente é utilizar passo adaptativo ou procurar métodos cujo a ordem seja maior.

A seleção de um método numérico para resolver uma EDO é justificada pela consistência, estabilidade e convergência. A seleção do tamanho de passo é importante pois se o passo é demasiado grande os métodos podem ser instáveis, a estabilidade é obtida quando o erro é propagado de forma decrescente, o contrário acontece quando o método é instável pois o erro propaga-se de forma crescente. 


\subsection{Passo adaptativo Método de Runge-Kutta-Fehlberg}

Uma forma de conseguir uma melhor precisão com um custo computacional baixo é diminuir ou aumentar o tamanho de passo selecionando um tamanho adequado o controle de passo consiste em tratar de manter constante o erro. E uma técnica consiste em rejeitar um passo quando o erro obtido é maior que a tolerância. O resultado disto pode ser amostrado na Figura 3.3. Pode-se

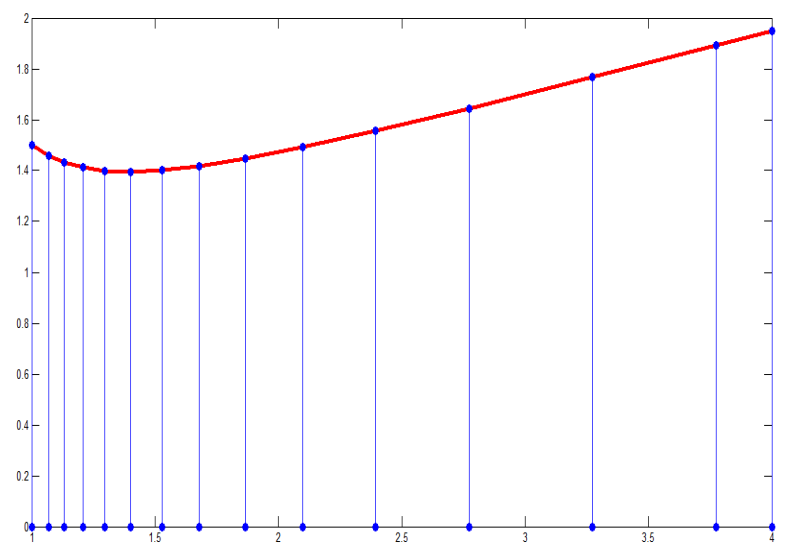

Figura 3.3: Passo adaptativo

descrever um algoritmo que faça a seleção do passo automaticamente. Existem diferentes algoritmos que resolvem a adaptatividade como por exemplo o método de Runge-Kutta-Fehlberg. Este método também denotado por RK45 é uma forma de resolver o problema de passo adaptativo devido a que tem incluído um critério para determinar se o tamanho de passo é adequado. Em cada iteração são calculadas duas aproximações distintas da solução para ser comparadas, no caso que os valores obtidos sejam similares aceitamos a aproximação e aumentamos ou mantemos o passo e caso de não coincidir com a precisão especificada temos que reduzir o tamanho de passo.

Para cada iteração temos que calcular os seguintes valores, que correspondem aos termos dos métodos de Runge-Kutta de ordem 4 e ordem 5:

$$
\begin{aligned}
k_{1} & =h f\left(t_{k}, y_{k}\right) \\
k_{2} & =h f\left(t_{k}+\frac{1}{4} h, y_{k}+\frac{1}{4} k_{1}\right), \\
k_{3} & =h f\left(t_{k}+\frac{3}{8} h, y_{k}+\frac{3}{32} k_{1}+\frac{9}{32} k_{2}\right), \\
k_{4} & =h f\left(t_{k}+\frac{12}{13} h, y_{k}+\frac{1932}{2197} k_{1}+\frac{7200}{2197} k_{2}+\frac{7296}{2197} k_{3}\right), \\
k_{5} & =h f\left(t_{k}+h, y_{k}+\frac{439}{216} k_{1}-8 k_{2}+\frac{3680}{513} k_{3}-\frac{845}{4104} k_{4}\right), \\
k_{6} & =h f\left(t_{k}+\frac{1}{2} h, y_{k}-\frac{8}{27} k_{1}+2 k_{2}-\frac{3544}{2565} k_{3}+\frac{1859}{4104} k_{4}-\frac{11}{40}\right) .
\end{aligned}
$$


Para efetuar o algoritmo achamos uma solução aproximada do problema de valor inicial usando um método de Runge-Kutta de ordem 4, usando a formula seguinte

$$
y_{k+1}=y_{k}+\frac{25}{216} k_{1}+\frac{1408}{2565} k_{3}+\frac{2197}{4101} k_{4}-\frac{1}{5} k_{5}
$$

a seguir logo calculamos uma aproximação usando um método Runge-Kutta de ordem 5 usando a fórmula

$$
z_{k+1}=y_{k}+\frac{16}{135} k_{1}+\frac{6656}{12825} k_{3}+\frac{28561}{56430} k_{4}-\frac{9}{50} k_{5}+\frac{2}{55} k_{6} .
$$

Assim o tamanho de passo ótimo determina-se por $s h$, multiplicando o tamanho de passo atual $h$ por un escalar $s$ dado por

$$
s=\left(\frac{\tau h}{2\left|z_{k+1}-y_{k+1}\right|}\right)^{1 / 4} \approx 0.84\left(\frac{\tau h}{\left|z_{k+1}-y_{k+1}\right|}\right)^{1 / 4}
$$

sendo $\tau$ a tolerância para controlar o erro que tem que ser especificada. 


\subsection{Métodos Lineares Multi-passo}

Quando são desenvolvidos os métodos de um passo tem-se que para determinar uma aproximação utiliza-se só o passo prévio, no entanto os passos anteriores podem ser aproveitados para calcular o novo passo. Se um método numérico que resolve uma EDO utiliza a informação dos passos prévios e com eles acha-se o passo seguinte denominam-se método multi-passo. Os métodos de passo-múltiplo ou multi-passo, podem-se obter de diferentes maneiras por exemplo por desenvolvimento de Taylor, integração numérica, interpolação, entre outros.

Em um método de um passo para efetuar as iterações $m=1,2, \ldots$ precisamos de um valor inicial $x_{0}=x(0)$. No caso de um método de $k$-passos para gerar as iterações $m=k, k+1, \ldots$ precisa-se de $k$ valores iniciais $x_{0}, x_{1}, \ldots, x_{k-1}$

Definição 3.3.1 (Método Multi-passo) Um método multi-passo de k-passos está dado pela seguinte relação

$$
\sum_{j=0}^{k} \alpha_{j} x_{n-j}=h \sum_{j=0}^{k} \beta_{j} f_{n-j}
$$

onde $\alpha_{j}, \beta_{j}$ são os coeficientes do método. Assumindo que $\alpha_{0} \neq 0$, e que $\left|\alpha_{k}\right|+\left|\beta_{k}\right| \neq 0$. Para evitar ambiguidade assumimos que $\alpha_{0}=1$ assim o método multi-passo é denominado explícito se $\beta_{0}=0$ e implícito quando $\beta_{0} \neq 0$

Um exemplo de método multi-passo é o Método de Adams-Bashforth cujas fórmulas para 3 e 4 passos estão dadas por

\section{Método de Adams-Bashforth de 3 passos}

$$
x_{i+1}=x_{i}+\frac{h}{12}\left(23 f_{i}-16 f_{i-1}+5 f_{i-2}\right)
$$

\section{Método de Adams-Bashforth de 4 passos}

$$
x_{i+1}=x_{i}+\frac{h}{24}\left(55 f_{i}-59 f_{i-1}+37 f_{i-2}-9 f_{i-3}\right)
$$


Métodos BDF Os métodos BDF, são métodos multi-passo. E estes métodos que são implícitos (Backward Differentiation Formulae). Um método BDF de k-passos é dado pela seguinte relação

$$
\sum_{i=0}^{k} \alpha_{i} x_{n-i}=h \beta_{0} f
$$

Por exemplo, a fórmula para o método BDF de terceira ordem é dada por

$$
x_{i+1}=\frac{18}{11} x_{i}-\frac{9}{11} x_{i-1}+\frac{2}{11} x_{i-2}+\frac{6}{11} h f_{i+1}
$$

como métodos BDF são implícitos, geralmente são implementados junto com o método de Newton modificado para resolver sistemas não lineares em cada passo.

\subsection{Método Previsor-Corretor de Adams-Bashforth- Moul- ton}

Quando se faz uma combinação de um método linear de passos múltiplos com um método implícito obtemos um método previsor-corretor, o método explicito prevê uma aproximação e o método implícito corrige essa previsão.

Este método é do tipo previsor corretor de passo múltiplo, para obter os valores inicias pode-se usar os métodos de um passo como por exemplo Runge-Kutta. Como exemplo podemos utilizar o método explicito de Adams-Bashforth de k-passos como método previsor e o método de AdamsMoulton de (k-1)-passos como método corretor. As seguintes fórmulas permitem efetivar o método

- Método explicito de Adams-Bashforth

$$
\begin{gathered}
x_{0}=\alpha, x_{1}=\alpha_{1}, x_{2}=\alpha_{2}, x_{3}=\alpha_{3} \\
x_{i+1}=x_{i}+\frac{h}{24}\left[55 f\left(t_{i}, x_{i}\right)-59 f\left(t_{i-1}, x_{i-1}\right)+37 f\left(t_{i-2}, x_{i-2}\right)-9 f\left(t_{i-3}, x_{i-3}\right)\right] \\
i=3,4, \ldots, n-1
\end{gathered}
$$

- Método implícito de Adams-Moulton

$$
\begin{gathered}
x_{0}=\alpha, x_{1}=\alpha_{1}, x_{2}=\alpha_{2} \\
x_{i+1}=x_{i}+\frac{h}{24}\left[9 f\left(t_{i+1}, x_{i+1}\right)+19 f\left(t_{i}, x_{i}\right)-5 f\left(t_{i-1}, x_{i-1}\right)+f\left(t_{i-2}, x_{i-2}\right)\right] \\
i=2,3, \ldots, n-1
\end{gathered}
$$




\subsection{Método Previsor-corretor de Euler-Newton}

Para achar o previsor usamos o método de Euler aplicado ao sistema de equações diferenciais ordinárias dado pelo sistema característico

$$
\left\{\begin{array}{l}
Y^{\prime}=g(s, Y), \quad s \in[0, a] \\
Y(0)=Y_{0}
\end{array}\right.
$$

logo na fase corretora aplicamos o método de Newton na função $F$ que define implicitamente a EDP por $F\left(x, y, u, u_{x}, u_{y}\right)=0$

- Euler

$$
Y_{i+1}=Y_{i}+h g(s, Y)
$$

- Newton

$$
W^{k+1}=W^{k}-\frac{F\left(W^{k}\right) \nabla F\left(W^{k}\right)}{\left\|\nabla F\left(W^{k}\right)\right\|^{2}}
$$

No entanto é possível usar na fase previsora qualquer método de maior ordem como por exemplo o método de Runge-Kutta

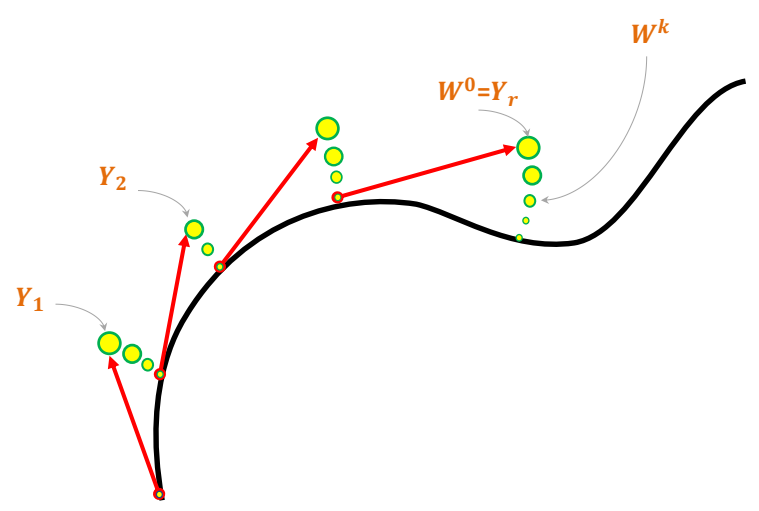

Figura 3.4: Método Previsor- Corretor 

Neste capítulo mostraremos a resolução numérica de uma EDP implícita de primeira ordem para o caso em que a função solução dependa de duas variáveis utilizando diferentes tipos de métodos e técnicas numéricas, faremos uma comparação dos resultados obtidos com a solução analítica utilizando tabelas que calculem o erro cometido assim como uma representação visual.

Se utilizamos métodos numéricos para resolver o sistema característico dado por (4.1)

$$
\left\{\begin{array}{l}
\frac{d X}{d t}=F_{p} \\
\frac{d Y}{d t}=F_{q} \\
\frac{d U}{d t}=p F_{p}+q F_{q} \\
\frac{d P}{d t}=-F_{x}-p F_{u} \\
\frac{d Q}{d t}=-F_{y}-q F_{u}
\end{array}\right.
$$

verifica-se que o erro global aumenta gradativamente (Ascher e Petzold, 1998).

Uma alternativa é a utilização de métodos numéricos para resolver o sistema característico (4.1) juntamente com um método de tipo projeção para retornar a solução a variedade original $F\left(x, y, u, u_{x}, u_{y}\right)=0$ como um método do tipo previsor corretor (Hairer e Wanner, 1993) .

Para realizar os diferentes gráficos deste trabalho foram desenvolvidos diversos códigos e interfaces em Matlab, o método Marching Tetrahedra (George e Frey, 2008; Gomes e Galbraith, 2009) foi implementado para reconstruir uma função implícita e assim mostrar e comparar a solução de uma EDP implícita com seu solução analítica, as interfaces implementadas tem uma forma interativa de uso para que seja fácil mudar dados, assim como os parâmetros das equações. 
Dada uma EDP implícita de primeira ordem $F\left(x_{1}, x_{2}, u, u_{x_{1}}, u_{x_{2}}\right)=0$, cuja parametrização da superfície solução é determinada por

$$
\Gamma(s, t)=\left(X_{1}(s, t), X_{2}(s, t), U(s, t)\right)
$$

como mostramos na Figura 4.1

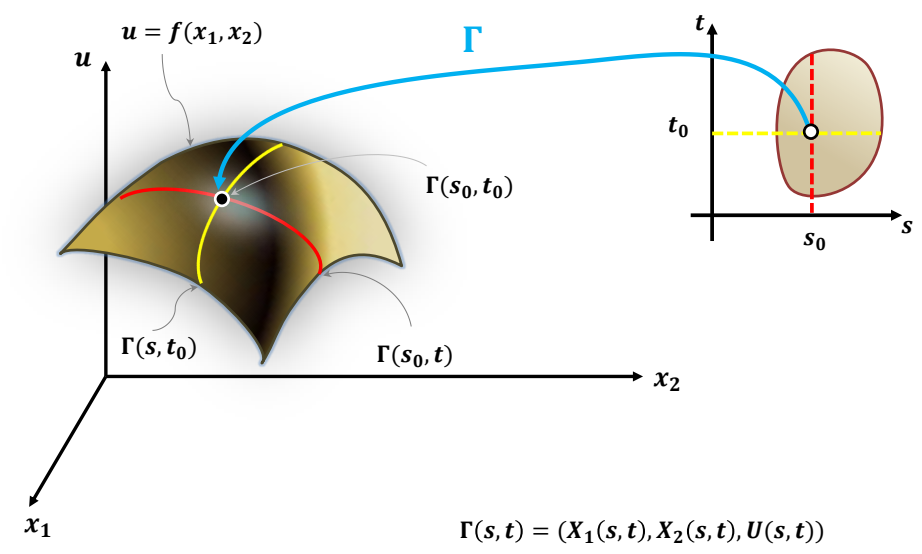

Figura 4.1: Superfície paramétrica

Uma curva inicial é definida como

$$
\Gamma(0, t)=\left(X_{1}(0, t), X_{2}(0, t), U(0, t)\right)=\left(X_{1}(t), X_{2}(t), U(t)\right)=\alpha(t)
$$

suas curvas características estão dadas por

$$
\Gamma\left(s, t_{j}\right)=\left(X_{1}\left(s, t_{j}\right), X_{2}\left(s, t_{j}\right), U\left(s, t_{j}\right)\right)=\left(X_{1}(s), X_{2}(s), U(s)\right)=c_{j}(s), \quad \operatorname{com} t_{j} \in[0, a]
$$

e um ponto da superfície parametrizada esta dado por

$$
\Gamma\left(s_{i}, t_{j}\right)=\left(X_{1}\left(s_{i}, t_{j}\right), X_{2}\left(s_{i}, t_{j}\right), U\left(s_{i}, t_{j}\right)\right), \quad \operatorname{com} t_{j} \in[0, a] \text { e } s_{i} \in[0, b]
$$

nosso objetivo é achar numericamente a superfície solução paramétrica para isso usaremos o seguinte sistema de equações denominado, sistema característico

$$
\begin{cases}\frac{d X_{1}}{d s}=F_{P_{1}}, & X_{1}(0)=x_{1}{ }^{0} \\ \frac{d X_{2}}{d s}=F_{P_{2}}, & X_{2}(0)=x_{2}{ }^{0} \\ \frac{d U}{d s}=P_{1} F_{P_{1}}+P_{2} F_{P_{2}}, & U(0)=u^{0} \\ \frac{d P_{1}}{d s}=-F_{X_{1}}-P_{1} F_{U}, & P_{1}(0)=p_{1}{ }^{0} \\ \frac{d P_{2}}{d s}=-F_{X_{2}}-P_{2} F_{U}, & P_{2}(0)=p_{2}{ }^{0}\end{cases}
$$




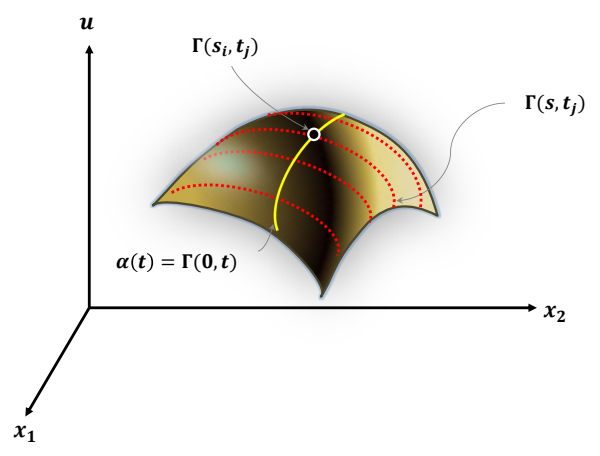

Figura 4.2: Curvas características

que permitirá achar as curvas características $c_{j}(s)$, estas curvas podem-se achar discretizando seu intervalo de definição $[0, b]$, assim uma curva ficaria dada por

$$
c_{j}\left(s_{i}\right)=\left(X_{1}\left(s_{i}\right), X_{2}\left(s_{i}\right), U\left(s_{i}\right)\right)=\left(X_{1}\left(s_{i}, t_{j}\right), X_{2}\left(s_{i}, t_{j}\right), U\left(s_{i}, t_{j}\right)\right)=\Gamma\left(s_{i}, t_{j}\right), s_{i} \in[0, b]
$$

por outro lado cada ponto de uma curva característica deve verificar a EDP implícita, isto é

$$
F\left(X_{1}\left(s_{i}, t_{j}\right), X_{2}\left(s_{i}, t_{j}\right), U\left(s_{i}, t_{j}\right), P_{1}\left(s_{i}, t_{j}\right), P_{2}\left(s_{i}, t_{j}\right)\right)=0 .
$$

Em geral os métodos numéricos que utilizaremos permitirão encontrar as "curvas solução" usando a ideia do método das caraterísticas, para isso a EDP será transformada num sistema de EDOs que permitirá achar as ditas curvas.

Os algoritmos desenvolvidos para resolver a EDP podem ser diferenciados em dois tipos: os algoritmos que resolvem a EDP que usam somente o sistema caraterístico e os algoritmos de tipo projeção que resolvem a EDP usando o sistema característico projetando os pontos sobre a superfície solução.

Solução usando sistemas de EDOs: Para resolver o Sistema característico 4.2 utilizaremos alguns métodos tais como:

- Método de Euler

- Método de Runge-Kutta de quinta ordem

- Método de Runge-Kutta-Fehlberg (RK45)

- Método Previsor-corretor de Adams-Bashforth- Moulton 
Segundo seja o caso, alguns destes métodos mencionados podem ser interpretados geometricamente como nas Figuras 4.3(a), 4.3(b), 4.4

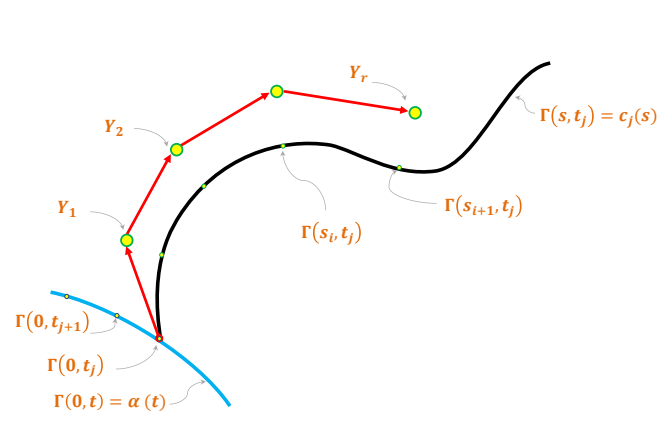

(a) Método de um Passo

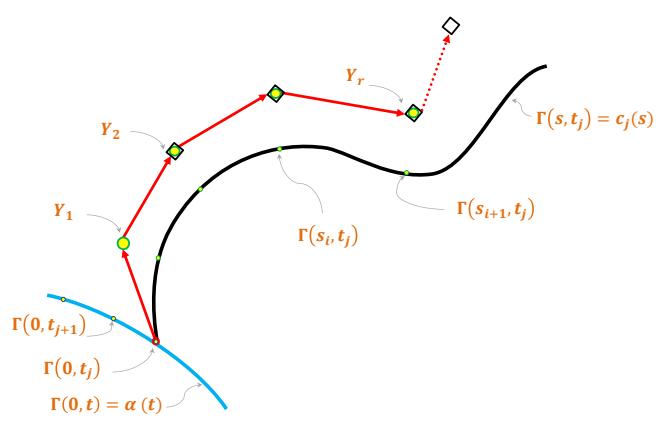

(b) Método Multi-passo

Figura 4.3: Soluções usando sistemas de ODEs

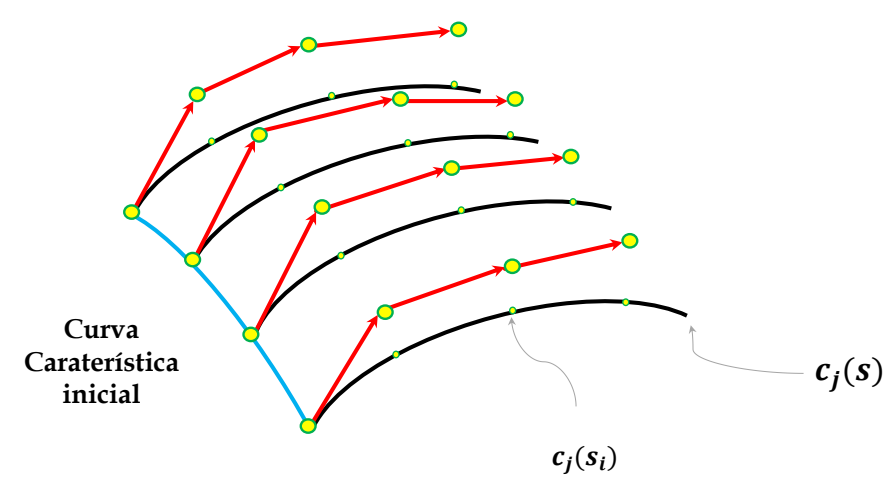

Figura 4.4: Curvas Características aproximadas pelo sistema Característico

Solução usando técnicas do tipo Projeção: Neste tipo de técnica utilizaremos um método para o qual o valor previsor pode ser achado do sistema característico (4.2) mediante um método de um passo e a projeção será desenvolvida na fase corretora mediante o método de Newton resolvendo a equação não linear $F=0$, tomando como valor inicial o previsor achado.

Se por exemplo usamos o método de Euler como previsor, o sistema característico dado por (4.2) pode ser denotado por

$$
\left\{\begin{array}{l}
Y^{\prime}=g(s, Y), \quad s \in[0, b] \\
Y(0)=Y_{0}
\end{array}\right.
$$


assim a formula iterativa descreve-se como

$$
Y_{i+1}=Y_{i}+h \cdot g(s, Y)
$$

onde

$$
\begin{gathered}
Y_{0}=\left[\begin{array}{c}
X_{1}(0) \\
X_{2}(0) \\
U(0) \\
P_{1}(0) \\
P_{2}(0)
\end{array}\right] \\
Y_{i}=\left[\begin{array}{c}
X_{1}\left(s_{i}\right) \\
X_{2}\left(s_{i}\right) \\
U\left(s_{i}\right) \\
P_{1}\left(s_{i}\right) \\
P_{2}\left(s_{i}\right)
\end{array}\right] \\
g(s, Y)=\left[\begin{array}{c}
F_{P_{1}} \\
F_{P_{2}} \\
P_{1} F_{P_{1}}+P_{2} F_{P_{2}} \\
-F_{X_{1}}-P_{1} F_{U} \\
-F_{X_{2}}-P_{2} F_{U}
\end{array}\right]
\end{gathered}
$$

No algoritmo que se desenvolverá mais adiante, $Y^{\prime}$ será denominado $V$ tang devido a que tem a direção do vetor tangente da curva $\left(X_{1}(s), X_{2}(s), U(s), P_{1}(s), P_{2}(s)\right)$, cuja projeção em $\mathbf{R}^{3}$ é a curva característica dada por $c(s)=\left(X_{1}(s), X_{2}(s), U(s)\right)$, a fórmula de Euler permite achar um a um os pontos da curva $c(s)$ usando o vetor tangente $V$ tang de uma curva característica o qual esta definido pelo sistema caraterístico, as diferentes curvas características acham-se fixando um ponto por vez da curva solução inicial.

Em cada iteração do Método de Euler, para cada ponto encontrado utiliza-se como método corretor o Método de Newton, para a função $F: \Omega \subset \mathbf{R}^{2} \times \mathbf{R} \times \mathbf{R}^{2} \rightarrow \mathbf{R}$ definida pela equação diferencial implícita $F\left(x_{1}, x_{2}, u, p_{1}, p_{2}\right)=0$, cujas iterações baseiam-se na formula

$$
W^{k+1}=W^{k}-\frac{F\left(W^{k}\right) \nabla F\left(W^{k}\right)}{\left\|\nabla F\left(W^{k}\right)\right\|^{2}}
$$

o ponto inicial do Método de Newton em cada etapa, é dado pelos valores do ponto preditor como na Figura (4.5), 4.6(a) assim para o preditor na iteração $k$-ésima temos que 


$$
W^{0}=Y_{r}=\left[\begin{array}{c}
X_{1}{ }^{r} \\
X_{2}{ }^{r} \\
U^{r} \\
P_{1}{ }^{r} \\
P_{2}{ }^{r}
\end{array}\right]
$$

na sequência de Newton o $k$-ésimo termo esta dado por

$$
W^{k}=\left[\begin{array}{c}
X_{1}{ }^{k} \\
X_{2}^{k} \\
U^{k} \\
P_{1}^{k} \\
P_{2}^{k}
\end{array}\right]
$$

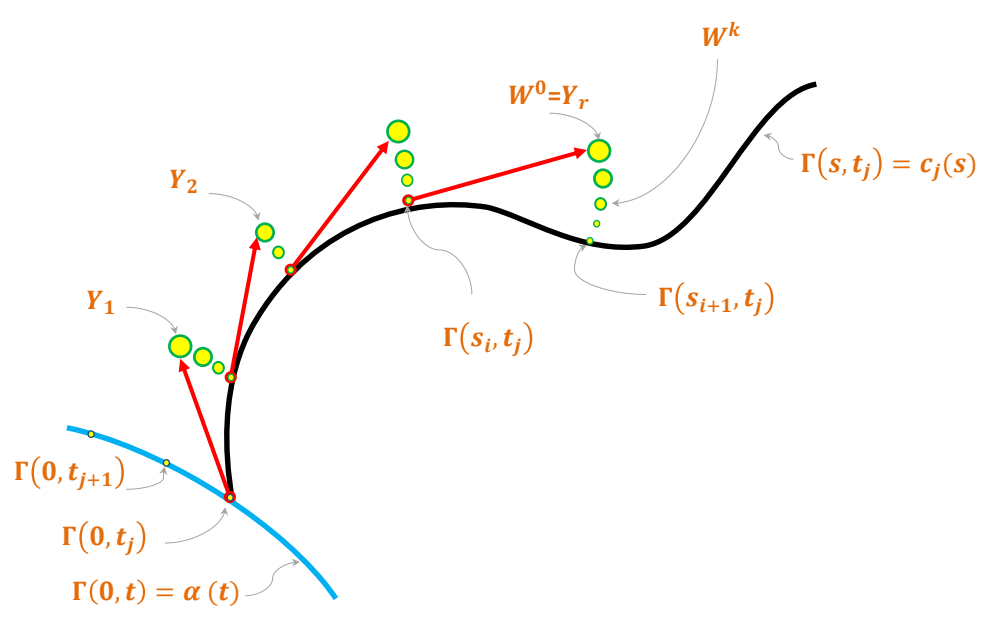

Figura 4.5: Curva solução inicial parametrizada e Curva característica parametrizada

Portanto mediante técnicas de métodos de continuação, propomos que tendo uma curva solução inicial sobre uma variedade, utilizar esta curva para achar outras curvas solução denominadas "curvas características"e assim achar a solução aproximada da equação (1.1). Uma representação gráfica da solução é dada pelo conjunto das curvas características assim como também poderia ser obtida como na Figura 4.6(b), mediante a triangulação dos pontos obtidos usando para isso alguma técnica conhecida de geração de malhas, como por exemplo Marching Tetrahedra (George e Frey, 2008; Gomes e Galbraith, 2009).

Utilizando um método de só um passo como por exemplo o método de Euler combinado com o método de Newton obtemos um algoritmo que resolve uma EDP implícita de primeira ordem para o caso de duas variáveis. 


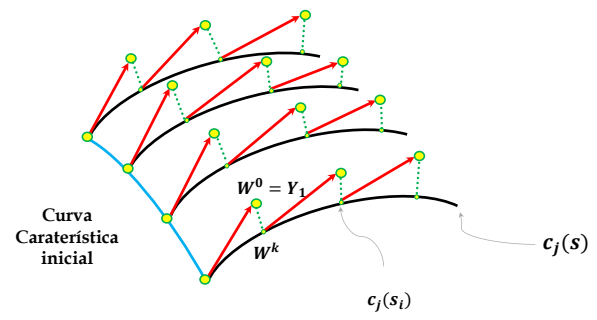

(a) Método Newton e previsor corretor

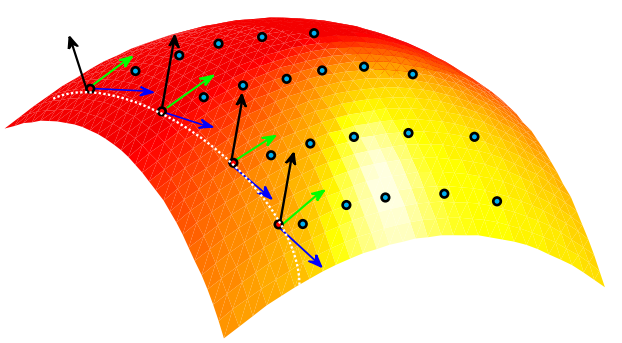

(b) Gráfico da solução

Figura 4.6: Geração da Superfície usando uma curva característica (solução inicial)

\section{ALGORITMO}

Passo 1: Definição de dados

- $F\left(x_{1}, x_{2}, u, p_{1}, p_{2}\right) \quad$ : Função implícita

- $\alpha$

- $N$

- $t_{0}$

- $a$

- $m$

- $t_{j}$

- $s_{0}$

- $b$

- $n$

- $h$

- $s_{i}$

- $Y_{0, j}$

- $Y_{i j}$

- $\operatorname{Vtang}\left(Y_{0}\right)$

- $t o l$
: Curva dado, $\alpha\left(t_{j}\right)=\left(X\left(t_{j}\right), Y\left(t_{j}\right), U\left(t_{j}\right)\right)$

: Vetores Normais $N=\left(P\left(t_{j}\right), Q\left(t_{j}\right),-1\right)$

: Ponto inicial do intervalo da solução numérica

: Ponto final do intervalo do domínio da curva dado

: Número de pontos da curva característica

: j-ésimo ponto do intervalo $\left[t_{0}, a\right], j=1, \ldots, m$

: Ponto inicial do intervalo da solução numérica

: Ponto final do intervalo da solução numérica

: Numero de sub-divisões do intervalo $\left[s_{0}, b\right]$

: Amplitude de passo $h=\left(b-s_{0}\right) / n$

$: s_{i}=s_{0}+i h, \quad i=1, \ldots, n$

: Ponto inicial da curva $c_{j}(s), j=0,1,2, \ldots m$

$$
Y_{0, j}=\left(X\left(0, t_{j}\right), Y\left(0, t_{j}\right), U\left(0, t_{j}\right), P\left(0, t_{j}\right), Q\left(0, t_{j}\right)\right)
$$

: i-ésimo ponto da curva $c_{j}(s), j=0,1,2, \ldots m$

$$
Y_{i, j}=\left(X\left(s_{i}, t_{j}\right), Y\left(s_{i}, t_{j}\right), U\left(s_{i}, t_{j}\right), P\left(s_{i}, t_{j}\right), Q\left(s_{i}, t_{j}\right)\right)
$$

:Vetor $g(s, Y)$ definido em (4.3) avaliado no vetor $Y=Y_{0}$

:Tolerância permitida na iteração de Newton $t o l=10^{-4}$

Passo 1.1 
Desde $j=1$ até $m$ fazer

$$
\begin{aligned}
& i=0 \\
& s=s_{0} \\
& Y_{0}=Y_{0, j}
\end{aligned}
$$

\section{Passo 2:}

$$
\begin{aligned}
& \text { Enquanto } s<b \\
& \qquad \begin{array}{l}
i=i+1 \\
Y_{1}=Y_{0}+h \cdot \operatorname{Vtang}\left(Y_{0}\right) \\
W^{0}=Y_{1} \\
k=0 ; \text { Erro }=\text { tol }+1
\end{array}
\end{aligned}
$$

Passo 2.1 Enquanto Erro $>$ tol

$$
\begin{aligned}
W^{k+1} & =W^{k}-\frac{F\left(W^{k}\right) \nabla F\left(W^{k}\right)}{\left\|\nabla F\left(W^{k}\right)\right\|^{2}} \\
& \text { Erro }=\left\|W^{k+1}-W^{k}\right\| \\
k=k+1 &
\end{aligned}
$$

Fim Passo 2.1

$$
\begin{aligned}
& Y_{i, j}=W^{k} \\
& s=s+h \\
& Y_{0}=Y_{1}
\end{aligned}
$$

Fim Passo 2

\section{Fim Passo 1.1}


Foram elaborados códigos no MATLAB usando interfaces gráficas para apresentar diferentes resultados que podem ser agrupados como segue:

- Curvas características de EDPs de primeira ordem: Esta interface permite fazer o gráfico de curvas características de algumas EDPs para comparar a solução analítica com a solução paramétrica.

- Solução numérica de EDPs de primeira ordem lineares e quase-lineares: A interface mostra como resolver EDPs usando um sistema de EDOs aproveitando a forma destas equações.

- Solução numérica de EDPs de primeira ordem Implícitas: Nesta interface desenvolvese o caso geral onde uma EDP pode ser linear ou não linear para isso utiliza-se o sistema caraterístico analogamente ao caso linear e quase-linear.

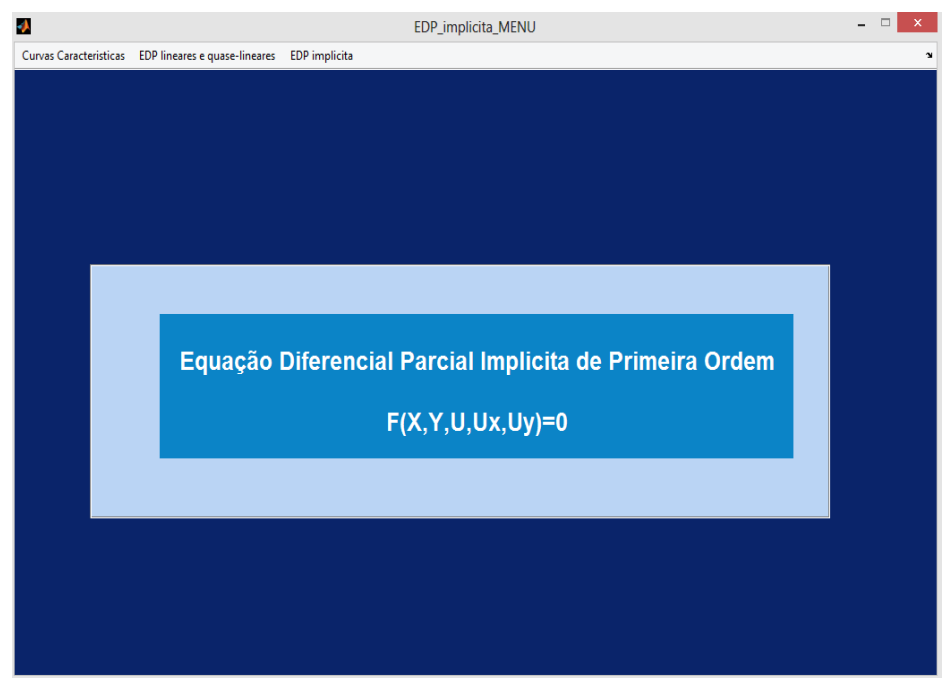

Figura 4.7: Interface no Matlab para o Menu de opções 


\subsection{Curvas características de EDPs de primeira ordem}

Os códigos elaborados nesta interface mostram a solução analítica de alguns casos particulares de EDPs de primeira ordem comparando suas curvas características com a solução.

Exemplo 4.1 A equação da forma $a u_{x}+u_{y}=0$, com condição inicial $u(x, 0)=f(x)$ tem como solução analítica $u(x, y)=f(x-a y)$, cujas curvas características são mostradas nas Figuras 4.8(a), 4.8(b).

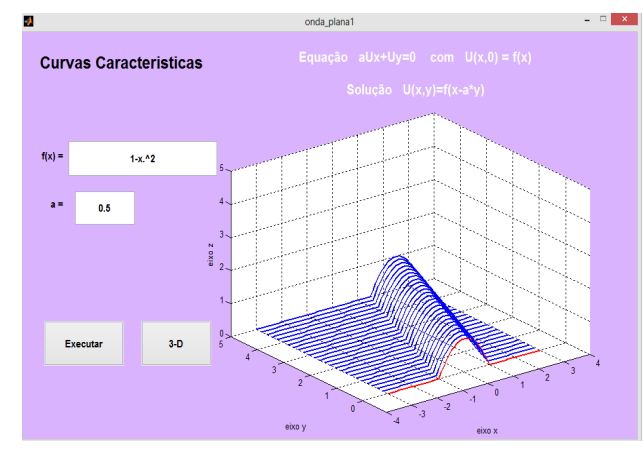

(a) Onda plana para $a=0.5$, e $f(x)=1-x^{2}$

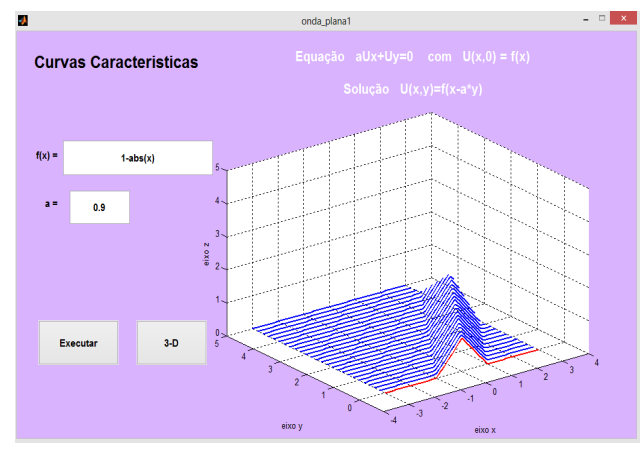

(b) Onda plana para $a=0.9$, e $f(x)=1-|x|$

Figura 4.8: Curvas características da equação $a u_{x}+u_{y}=0$

Exemplo 4.2 Para uma equação da forma $x u_{x}+y u_{y}=c u$ com condição inicial $u(x, 1)=f(x)$ a solução analítica é dada por $u(x, y)=f(x / y) y^{c}$. Para o caso em que $u(x, 1)=e^{-x^{2}}$ a superfície solução para $c=-1$ assim como as curvas características são dadas nas Figuras 4.9(a), 4.9(b), 4.10(a), 4.10(b) para o caso em que $c=-1$ temos as Figuras 4.11(a), 4.11(b), 4.12(a), 4.12(b).

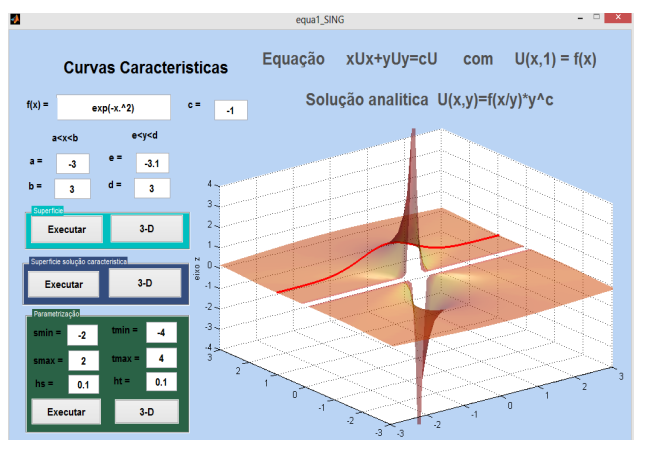

(a) Superficie solução de $x u_{x}+y u=c u$ na interface

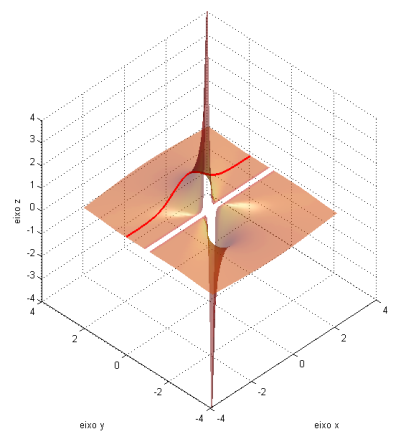

(b) Superficie solução de $x u_{x}+y u=c u$

Figura 4.9: Superfície solução da EDP $x u_{x}+y u=c u, c=-1$ e $u(x, 1)=e^{-x^{2}}$ 


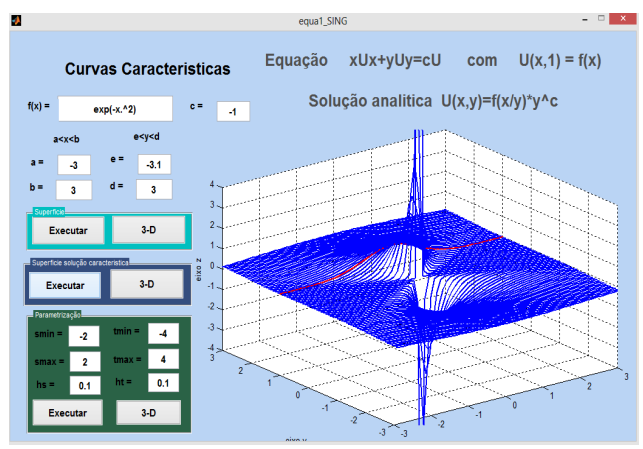

(a) Curvas características da EDP na interface

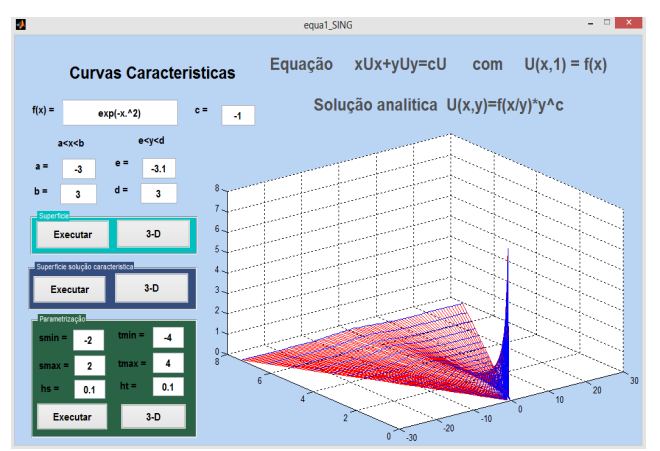

(b) Solução parametrica de $x u_{x}+y u=c u$

Figura 4.10: Curvas características da EDP $x u_{x}+y u=c u, c=-1$ e $u(x, 1)=e^{-x^{2}}$

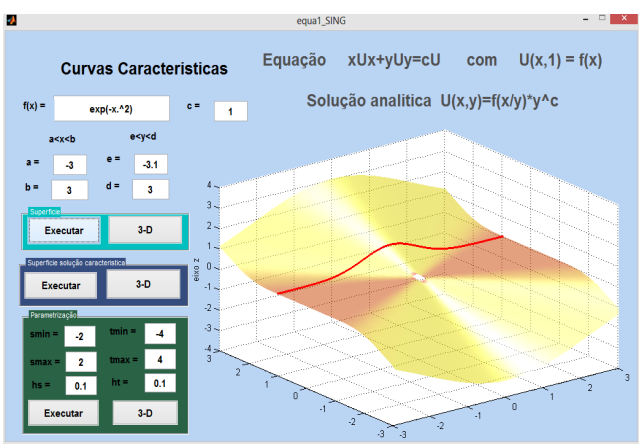

(a) Superficie solução da EDP $x u_{x}+y u=c u$ na interface

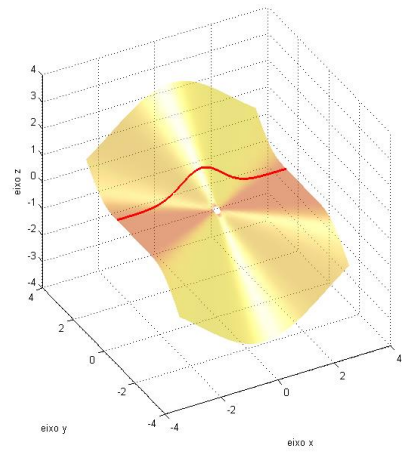

(b) Superficie solução de $x u_{x}+y u=c u$

Figura 4.11: Superfície solução da EDP $x u_{x}+y u=c u, c=1$ e $u(x, 1)=\exp \left(-x^{2}\right)$

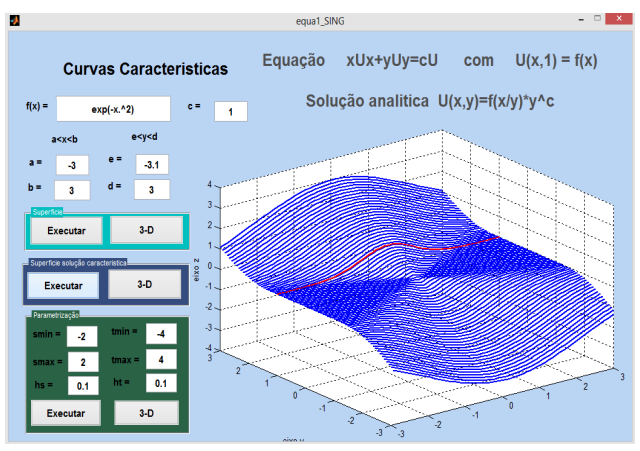

(a) Curvas características da EDP na interface

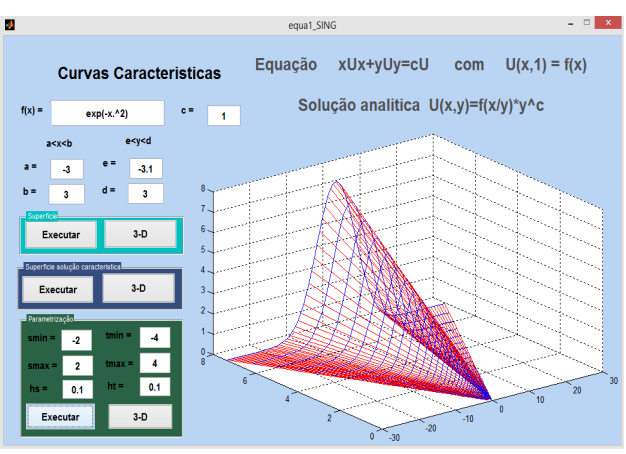

(b) Solução parametrica de $x u_{x}+y u=c u$

Figura 4.12: Curvas características da EDP $x u_{x}+y u=c u, c=1$ e $u(x, 1)=e^{-x^{2}}$ 


\subsection{Solução numérica de EDPs lineares e quase-lineares de primeira ordem}

Para resolver numericamente as EDPs lineares $R_{1}(x, y) u_{x}+R_{2}(x, y) u_{y}=T(x, y, u)$ ou EDPs quase-lineares $R_{1}(x, y, u) u_{x}+R_{2}(x, y, u) u_{y}=T(x, y, u)$, podemos estabelecer o sistema

$$
\begin{cases}\frac{d X}{d s}=R_{1} & X(0)=X(t) \\ \frac{d Y}{d s}=R_{2} & Y(0)=Y(t) \\ \frac{d U}{d s}=T & U(0)=U(t)\end{cases}
$$

que permite achar a solução numérica deste sistema de EDOs, por exemplo usando o Método de Runge-Kutta de quinta ordem para resolver o sistema obtemos os seguintes resultados.

Exemplo 4.3 Dada a EDP linear de primeira ordem

$$
\begin{gathered}
-y u_{x}+x u_{y}=4 x y \\
u(x, 0)=\cos (x)
\end{gathered}
$$

ao parametrizar a curva inicial $X(t)=t, Y(t)=0, U(t)=\cos (t)$, obtemos o sistema de EDOs

$$
\left\{\begin{array}{llrl}
\frac{d X}{d s} & =-y & & X(0)=t \\
\frac{d Y}{d s} & =x & & Y(0)=0 \\
\frac{d U}{d s} & =4 x y & & U(0)=\cos (t)
\end{array}\right.
$$

este sistema permite achar a solução numérica obtida é mostrada nas Figuras 4.13(a), 4.13(b).

Exemplo 4.4 Dada a EDP linear de primeira ordem

$$
\begin{gathered}
e^{y} u_{x}+u_{y}=u \\
u(x, 0)=\cos (x)
\end{gathered}
$$

ao parametrizar a curva inicial $X(t)=t, Y(t)=0, U(t)=\cos (t)$, obtemos as equações características

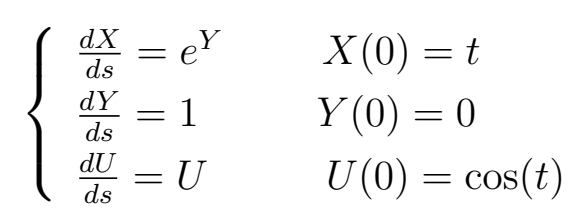

a solução numérica obtida é mostrada em nas Figuras 4.14(a), 4.14(b), e a solução analítica $u(x, y)=\cos \left(x-e^{y}+1\right) e^{y},\left\{(x, y) \in \mathbf{R}^{2}: x>e^{y}-1\right\}$ nas Figuras 4.15(a), 4.15(b). 


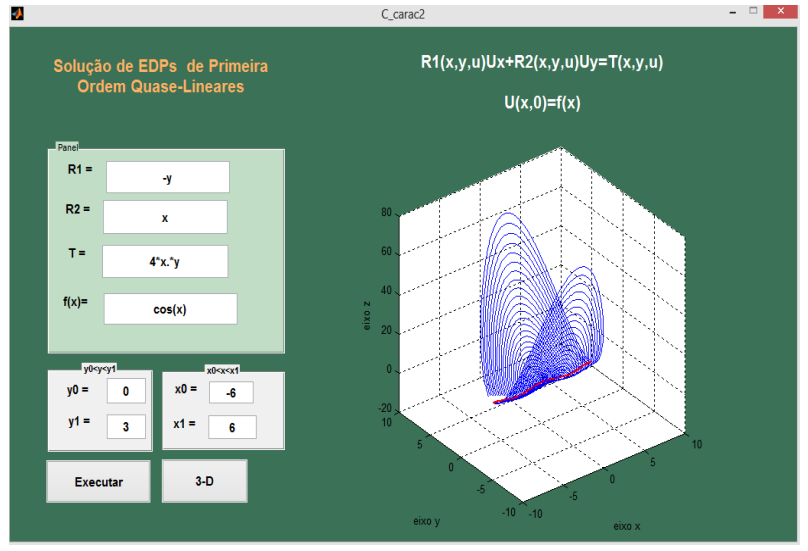

(a) Solução numerica na interface

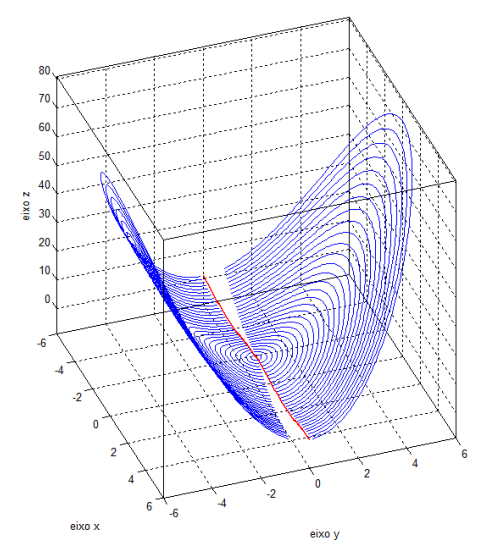

(b) Solução numérica

Figura 4.13: Solução Numérica da equação $-y u_{x}+x u_{y}=4 x y, \operatorname{com} u(x, 0)=\cos (x)$, usando Runge-Kutta de quinta ordem

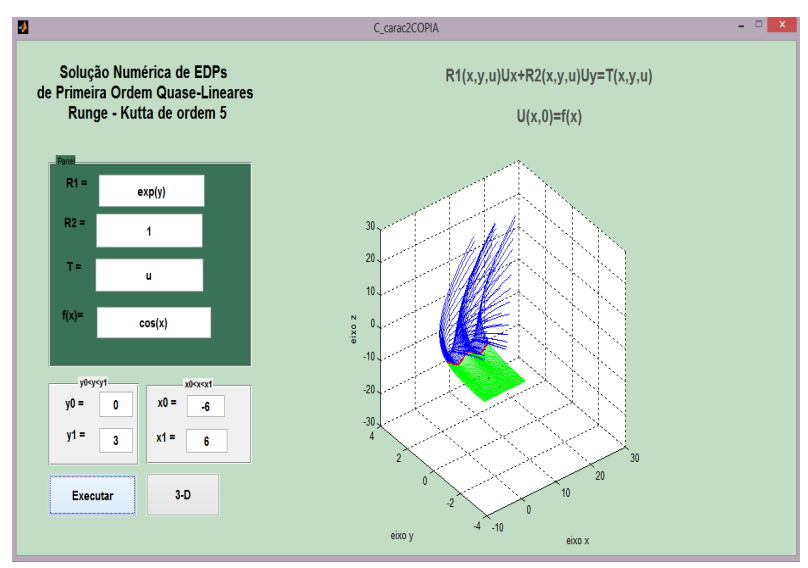

(a) Solução numerica na interface

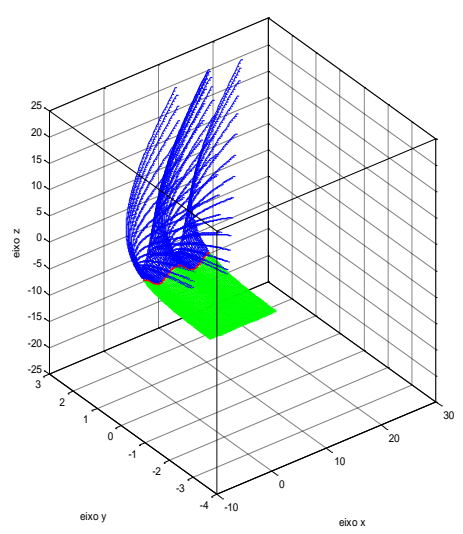

(b) Solução numérica

Figura 4.14: Solução Numérica da equação $e^{y} u_{x}+u_{y}=u$, $\operatorname{com} u(x, 0)=\cos (x)$, usando Runge-Kutta de quinta ordem

Exemplo 4.5 Dada a EDP quase-linear de primeira ordem

$$
\begin{gathered}
u u_{x}+u u_{y}=-x-y \\
u(x, 0)=\sqrt{1-x^{2}}
\end{gathered}
$$

ao parametrizar a curva inicial $X(t)=t, Y(t)=0, U(t)=\sqrt{1-t^{2}}$, obtemos as equações características

$$
\left\{\begin{array}{llrl}
\frac{d X}{d s} & =U & & X(0)=t \\
\frac{d Y}{d s}=U & Y(0)=0 \\
\frac{d U}{d s}=-X-Y & & U(0)=\sqrt{1-t^{2}}
\end{array}\right.
$$

a solução numérica obtida é mostrada em nas Figuras 4.16(a), 4.16(b),4.17(a), 4.17(b), sendo que sua solução analítica está contida na esfera $\quad x^{2}+y^{2}+u^{2}=1$. 


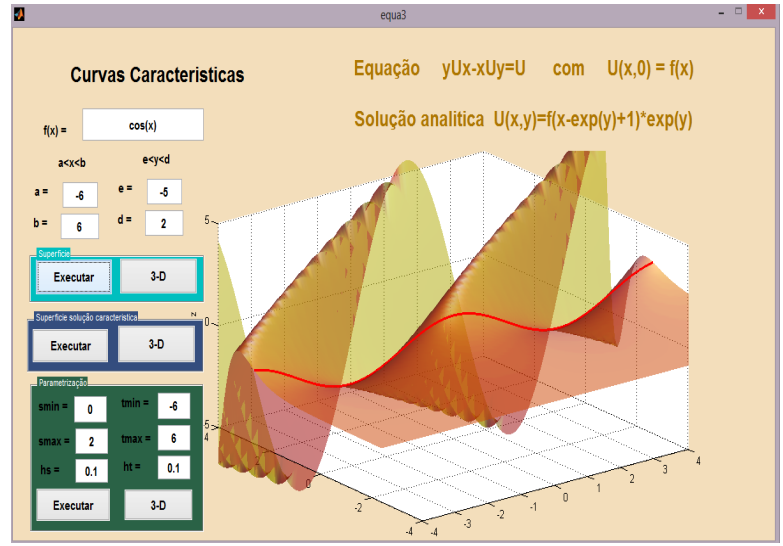

(a) Solução analítica na interface

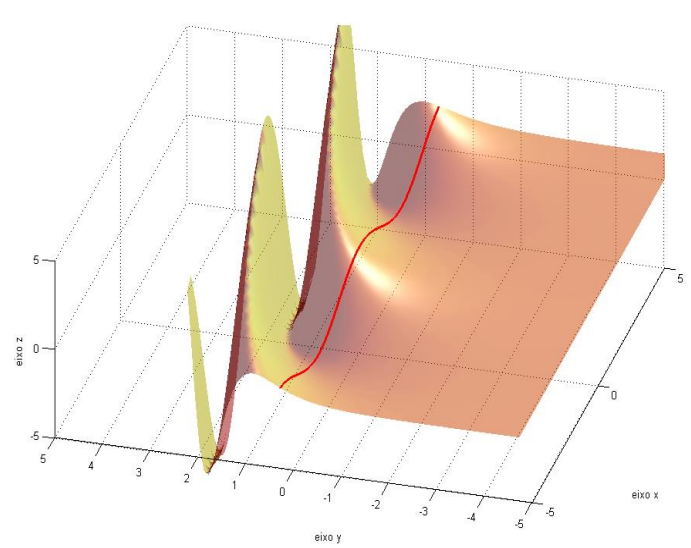

(b) Solução analítica

Figura 4.15: Solução analítica da equação $e^{y} u_{x}+u_{y}=u$

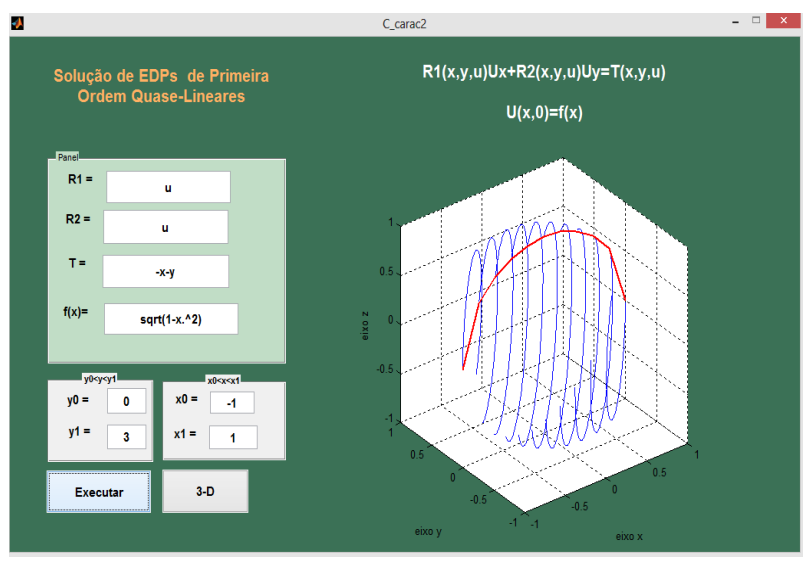

(a) Solução numerica na interface

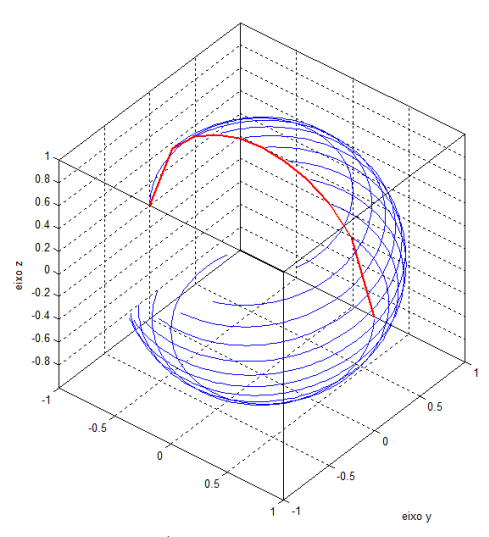

(b) Solução numérica

Figura 4.16: Solução Numérica de $u u_{x}+u u_{y}=-x-y$, com $u(x, 0)=\sqrt{1-x^{2}}$, usando Runge-Kutta de quinta ordem

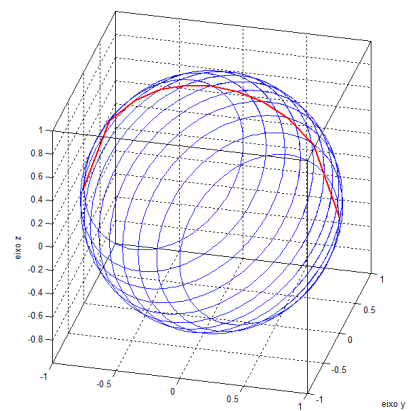

(a) Solução numérica num dominio maior

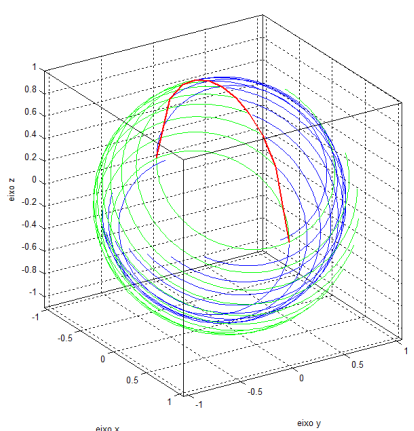

(b) Solução numérica bilateral

Figura 4.17: Solução numérica bilateral de $u u_{x}+u u_{y}=-x-y, \operatorname{com} u(x, 0)=\sqrt{1-x^{2}}$, usando Runge-Kutta de quinta ordem 


\subsection{Solução numérica de EDPs implícitas de primeira or- dem}

Para resolver numericamente uma EDP não linear de primeira ordem implícita utilizaremos a notação generalizada, descrevendo a EDP por

$$
F\left(x_{1}, x_{2}, u, p_{1}, p_{2}\right)=0
$$

sendo a curva inicial $\left(X_{1}(t), X_{2}(t), U(t)\right)$, com vetores normais $N=\left(P_{1}(t), P_{2}(t),-1\right)$ e o sistema Característico

$$
\left\{\begin{array}{l}
\frac{d X_{1}}{d s}=F_{p_{1}} \\
\frac{d X_{2}}{d s}=F_{p_{2}} \\
\frac{d U}{d s}=p_{1} F_{p_{1}}+p_{2} F_{p_{2}} \\
\frac{d P_{1}}{d s}=-F_{x_{1}}-p_{1} F_{u} \\
\frac{d P_{2}}{d s}=-F_{x_{2}}-p_{2} F_{u}
\end{array}\right.
$$

\section{MÉTODO DE EULER}

Exemplo 4.6 Dada a equação

$$
\begin{gathered}
\left(u_{x_{1}}\right)^{2}+\left(u_{x_{2}}\right)^{2}=1 \\
x_{1}{ }^{2}+x_{2}{ }^{2}=1, u=0
\end{gathered}
$$

Podemos expressar a equação como $F\left(x_{1}, x_{2}, u, u_{x_{1}}, u_{x_{2}}\right)=\left(u_{x_{1}}\right)^{2}+\left(u_{x_{2}}\right)^{2}-1=0$, ou equivalentemente $F\left(x_{1}, x_{2}, u, p_{1}, p_{2}\right)=p_{1}{ }^{2}+p_{2}{ }^{2}-1=0$, por outro lado sendo a condição inicial $x_{1}^{2}+x_{2}^{2}=1$, com $u=0$, ao parametrizar a curva inicial fica como $\alpha(t)=(\cos (t), \sin (t), 0)$, com vetores normais dados por $N(t)=(\cos (t), \sin (t),-1)$, logo usando o código desenvolvido, obtemos os resultados mostrados nas Figuras 4.18(a), 4.18(b).

Exemplo 4.7 Seja a equação

$$
\begin{gathered}
\left(u_{x_{1}}\right)^{2}+u_{x_{2}}+u=0 \\
u\left(x_{1}, 0\right)=x_{1}
\end{gathered}
$$

que pode ser expressa como $F\left(x_{1}, x_{2}, u, u_{x_{1}}, u_{x_{2}}\right)=\left(u_{x_{1}}\right)^{2}+u_{x_{2}}+u=0$, ou equivalentemente $F\left(x_{1}, x_{2}, u, p_{1}, p_{2}\right)=p_{1}{ }^{2}+p_{2}+u=0$, por outro lado sendo a condição inicial $u\left(x_{1}, 0\right)=x_{1}$, ao parametrizar a curva inicial fica como $\alpha(t)=(t, 0, t)$, com vetores normais dados por $N(t)=$ $(1,-(1+t),-1)$, logo usando o código desenvolvido, obtemos os seguintes resultados das Figuras 4.19(a), 4.19(b). 


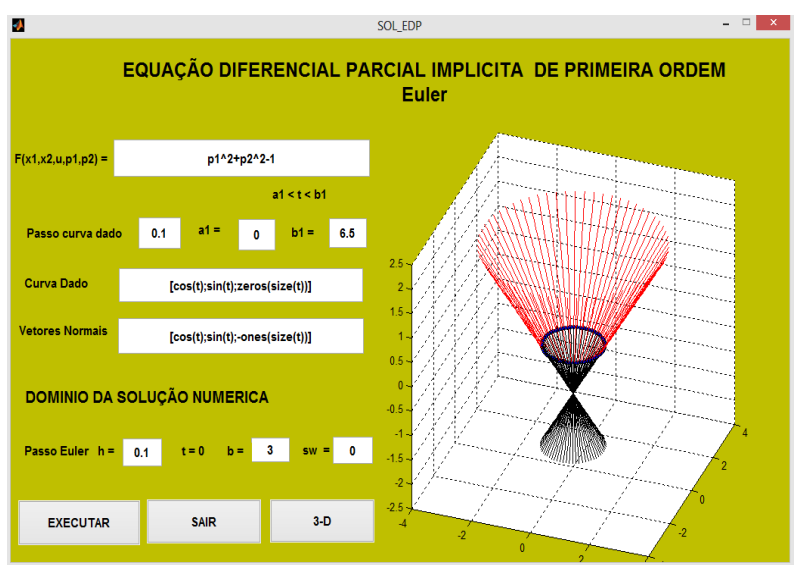

(a) Condições iniciais na interface

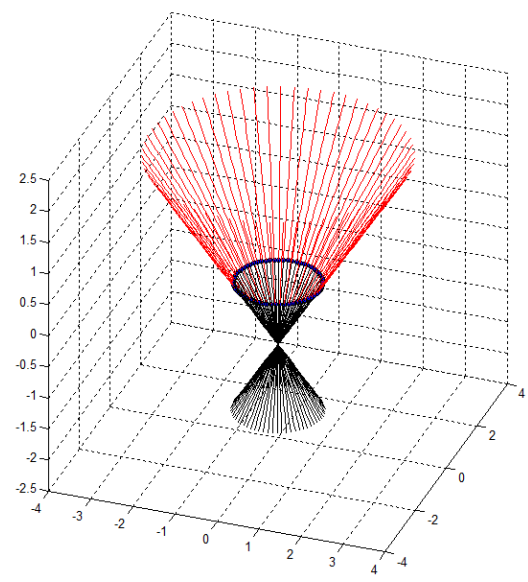

(b) Curvas características

Figura 4.18: Solução numérica da equação $\left(u_{x_{1}}\right)^{2}+\left(u_{x_{2}}\right)^{2}=1$, com condição inicial $x_{1}^{2}+x_{2}^{2}=1, u=0$, usando o Método de Euler

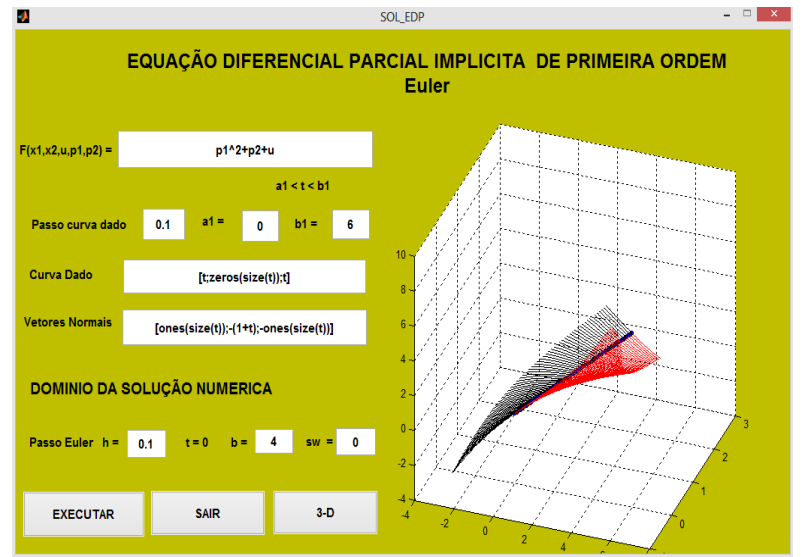

(a) Condições iniciais na interface

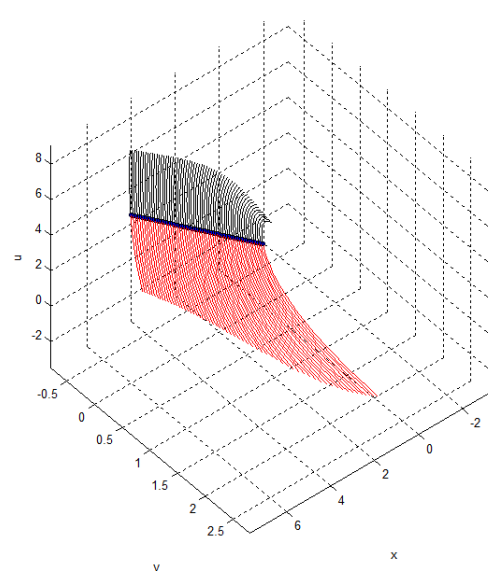

(b) Curvas características

Figura 4.19: Solução numérica da equação $u u_{x_{1}}+u_{x_{2}}=0$, com condição inicial $u\left(x_{1}, 0\right)=-x_{1}$, usando o Método de Euler

Exemplo 4.8 A esfera $x_{1}{ }^{2}+x_{2}{ }^{2}+u^{2}=1$, onde $u=f\left(x_{1}, x_{2}\right)$ pode expressar-se em termos de uma equação diferencial parcial implícita de primeira ordem $F\left(x_{1}, x_{2}, u, p_{1}, p_{2}\right)=x_{1}+x_{2}+$ $u p_{1}+u p_{2}=0$, sendo a condição inicial $x_{1}^{2}+u^{2}=1$, ao parametrizar a curva inicial fica como $\alpha(t)=(\cos (t), 0, \sin (t))$, com vetores normais dados por $N(t)=(-\cos (t) / \sin (t), 0,-1)$, logo usando o código desenvolvido, obtemos os seguintes resultados das Figuras 4.20(a), 4.20(b) 4.21(a), 4.21(b) 


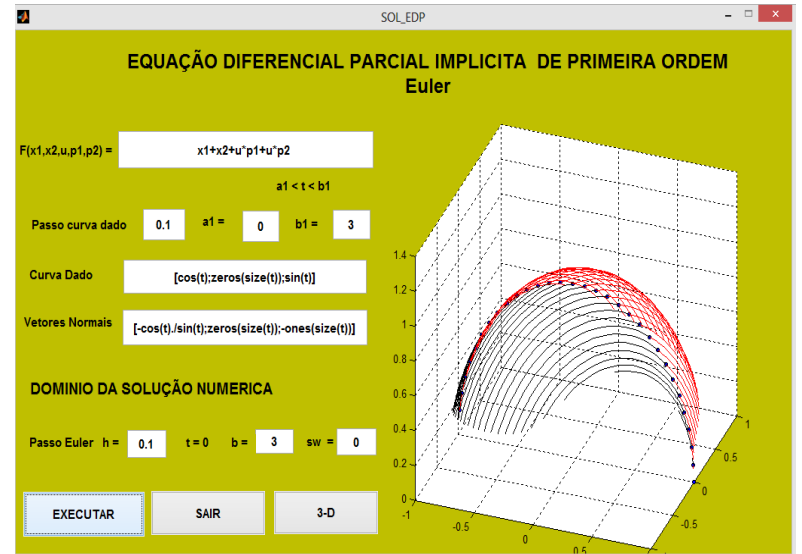

(a) Interface Runge-Kutta de ordem 5

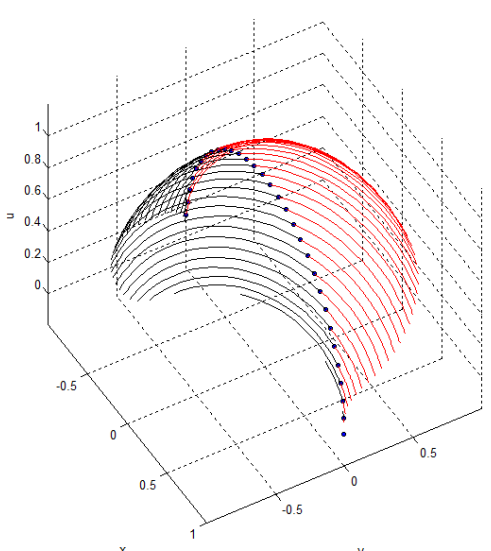

(b) Curvas características

Figura 4.20: Solução numérica da EDP $x_{1}+x_{2}+u p_{1}+u p_{2}=0, \operatorname{com} x_{1}{ }^{2}+u^{2}=1$, usando o Método de Euler

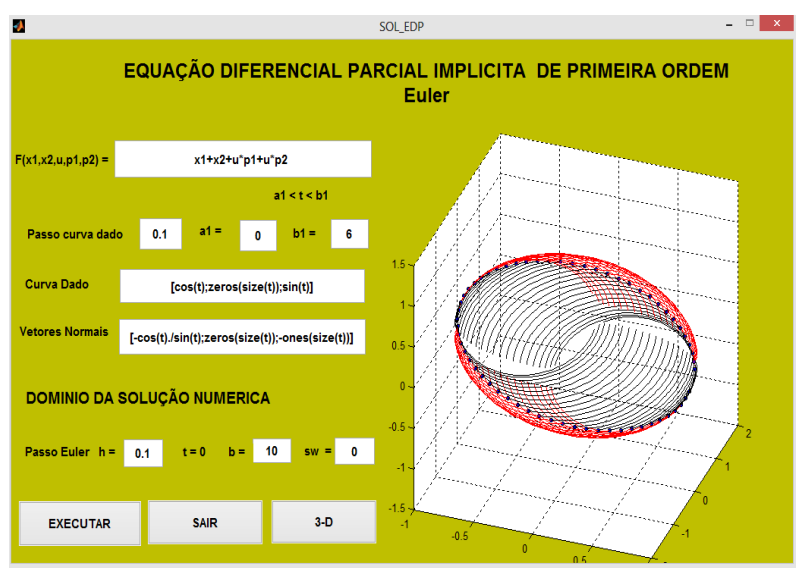

(a) Interface Runge-Kutta de ordem 5

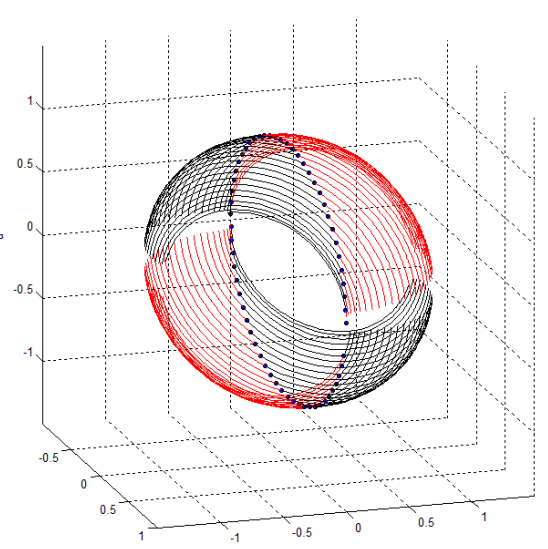

(b) Curvas características

Figura 4.21: Solução numérica da EDP $x_{1}+x_{2}+u p_{1}+u p_{2}=0$, com $x_{1}{ }^{2}+u^{2}=1$, usando o Método de Euler

\section{MÉTODO DE RUNGE-KUTTA DE QUINTA ORDEM}

Exemplo 4.9 Dada a equação

$$
\begin{gathered}
\left(u_{x_{1}}\right)^{2}+\left(u_{x_{2}}\right)^{2}=1 \\
x_{1}{ }^{2}+x_{2}{ }^{2}=1, u=0
\end{gathered}
$$

podemos expressar a equação como $F\left(x_{1}, x_{2}, u, u_{x_{1}}, u_{x_{2}}\right)=\left(u_{x_{1}}\right)^{2}+\left(u_{x_{2}}\right)^{2}-1=0$, ou equivalentemente $F\left(x_{1}, x_{2}, u, p_{1}, p_{2}\right)={p_{1}}^{2}+{p_{2}}^{2}-1=0$, por outro lado sendo a condição inicial $x_{1}{ }^{2}+x_{2}{ }^{2}=1$, com $u=0$, ao parametrizar a curva inicial fica como $\alpha(t)=(\cos (t), \sin (t), 0)$, com vetores normais dados por $N(t)=(\cos (t), \sin (t),-1)$, logo usando o código desenvolvido, obtemos os seguintes resultados das Figuras 4.22(a), 4.22(b). 


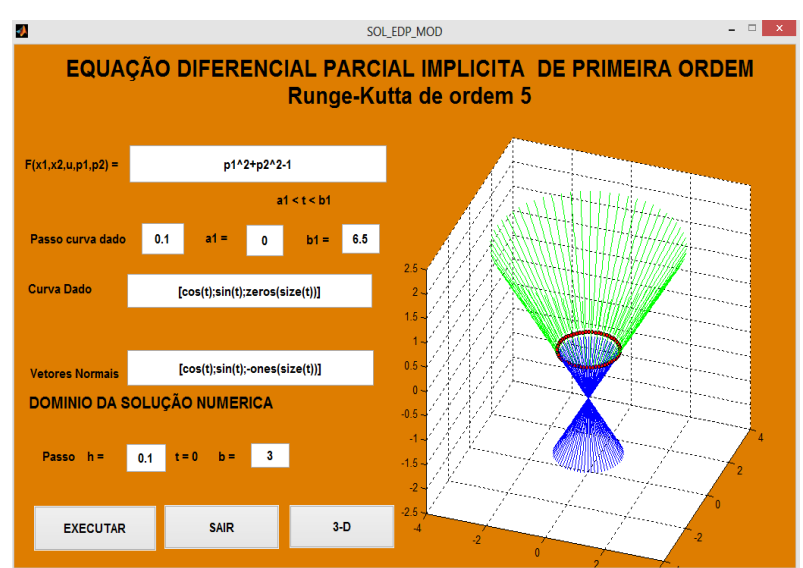

(a) Condições iniciais na interface

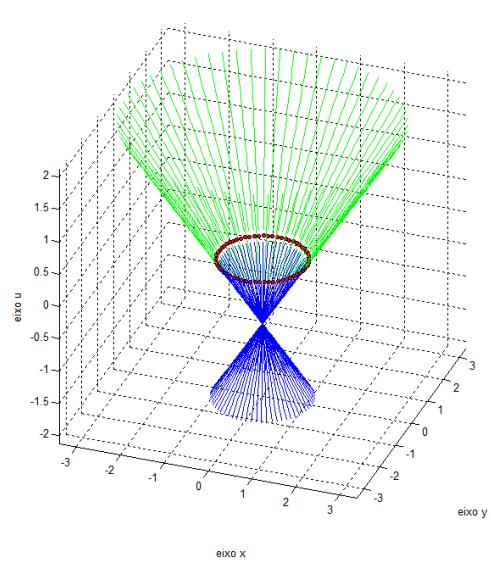

(b) Curvas características

Figura 4.22: Solução numérica da equação $\left(u_{x_{1}}\right)^{2}+\left(u_{x_{2}}\right)^{2}=1$, com condição inicial $x_{1}^{2}+x_{2}^{2}=1, u=0$, usando o Método de Runge-Kutta de ordem 5

Exemplo 4.10 A esfera $x_{1}{ }^{2}+x_{2}{ }^{2}+u^{2}=1$, onde $u=f\left(x_{1}, x_{2}\right)$ pode expressar-se em termos de uma equação diferencial parcial implícita de primeiro ordem $F\left(x_{1}, x_{2}, u, p_{1}, p_{2}\right)=x_{1}+x_{2}+$ $u p_{1}+u p_{2}=0$, ademas sendo a condição inicial $x_{1}^{2}+u^{2}=1$, ao parametrizar a curva inicial fica como $\alpha(t)=(\cos (t), 0, \sin (t))$, com vetores normais dados por $N(t)=(-\cos (t) / \sin (t), 0,-1)$, logo usando o código desenvolvido, obtemos os seguintes resultados das Figuras 4.23(a), 4.23(b), 4.24(a), 4.24(b).

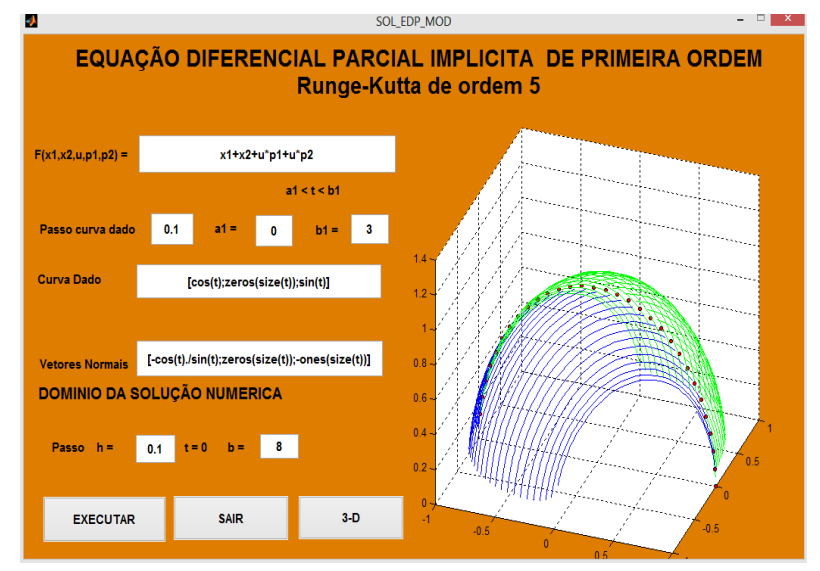

(a) Interface Runge-Kutta de ordem 5

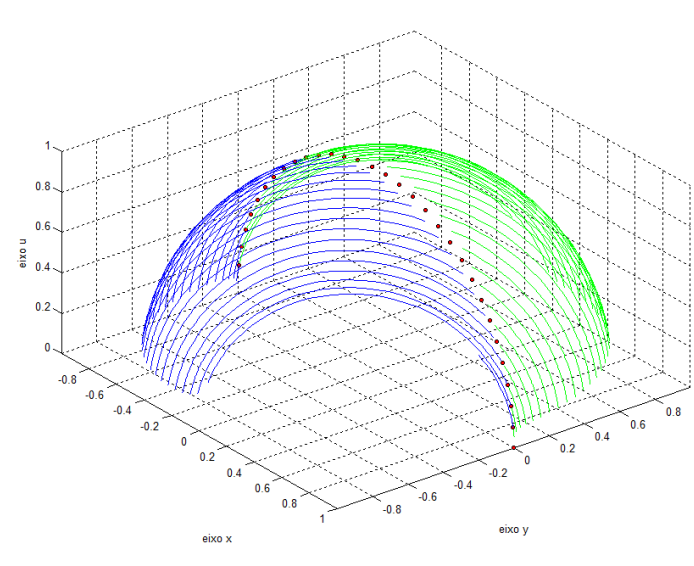

(b) Curvas características

Figura 4.23: Solução numérica da EDP $x_{1}+x_{2}+u p_{1}+u p_{2}=0, \operatorname{com} x_{1}^{2}+u^{2}=1$, usando o Método de Runge-Kutta de ordem 5

Exemplo 4.11 Dada a EDP implícita $F\left(x_{1}, x_{2}, u, p_{1}, p_{2}\right)=-x_{2} p_{1}+x_{1} p_{2}-4 x_{1} x_{2}=0$, com condição inicial $u\left(x_{1}, 0\right)=\cos \left(x_{1}\right)$, ao parametrizar a curva inicial fica como $\alpha(t)=(t, 0, \cos (t))$, com vetores normais dados por $N(t)=(-\sin (t), 0,-1)$, logo obtemos os seguintes resultados das Figuras 4.25(a), 4.25(b). 


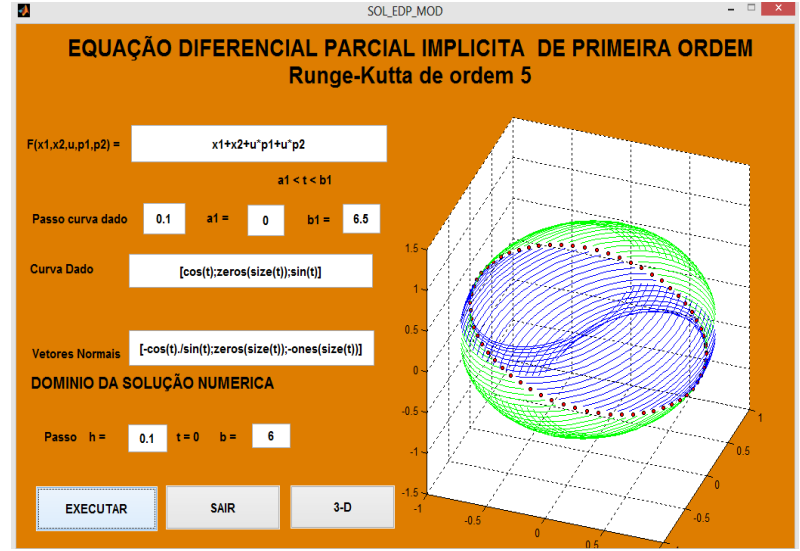

(a) Interface Runge-Kutta de ordem 5

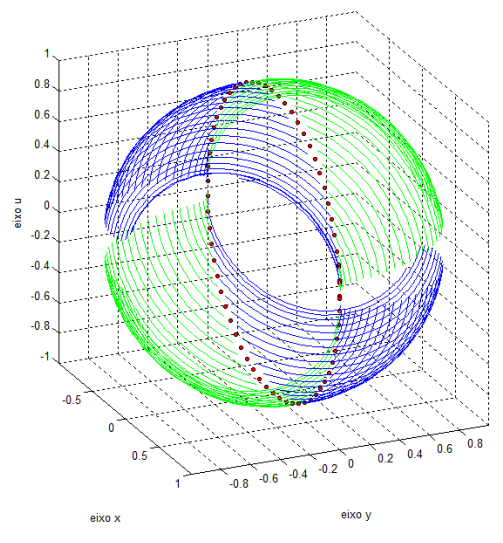

(b) Curvas características

Figura 4.24: Solução numérica da EDP $x_{1}+x_{2}+u p_{1}+u p_{2}=0$, com $x_{1}^{2}+u^{2}=1$, usando o Método de Runge-Kutta de ordem 5

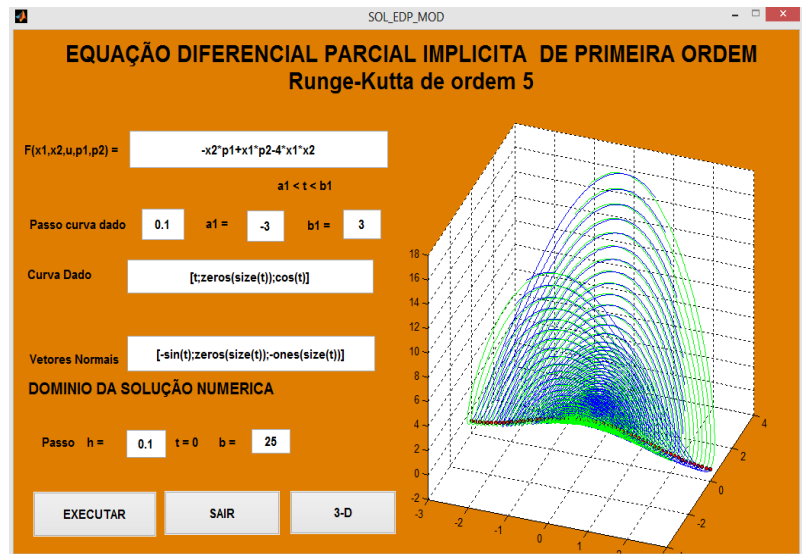

(a) Interface Runge-Kutta de ordem 5

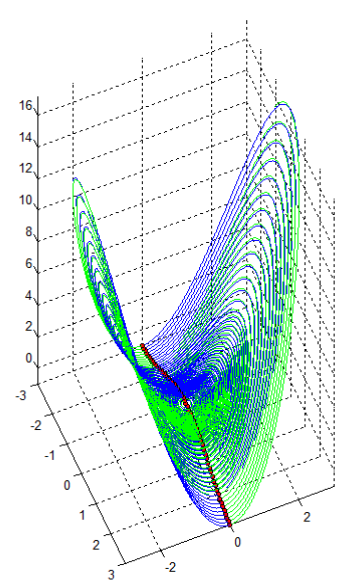

(b) Curvas características

Figura 4.25: Solução numérica da EDP $x_{2} p_{1}+x_{1} p_{2}-4 x_{1} x_{2}=0$, com $u\left(x_{1}, 0\right)=\cos \left(x_{1}\right)$, usando o Método de Runge-Kutta de ordem 5

\section{MÉTODO PREVISOR CORRETOR DE ADAMS-BASHFORTH-MOULTON DE PASSO MÚLTIPLO}

O algoritmo desenvolvido utiliza como valores iniciais os valores obtidos pelo método de Runge-Kutta de quarta ordem com passo constante.

Exemplo 4.12 Seja a equação

$$
\begin{gathered}
\left(u_{x_{1}}\right)^{2}+\left(u_{x_{2}}\right)^{2}=1 \\
x_{1}{ }^{2}+x_{2}{ }^{2}=1, u=0
\end{gathered}
$$

que na forma implícita expressa-se como $F\left(x_{1}, x_{2}, u, p_{1}, p_{2}\right)=\left(u_{x_{1}}\right)^{2}+\left(u_{x_{2}}\right)^{2}-1=0$, a condição inicial fica parametrizada como $\alpha(t)=(\cos (t), \sin (t), 0)$, sendo os vetores normais 


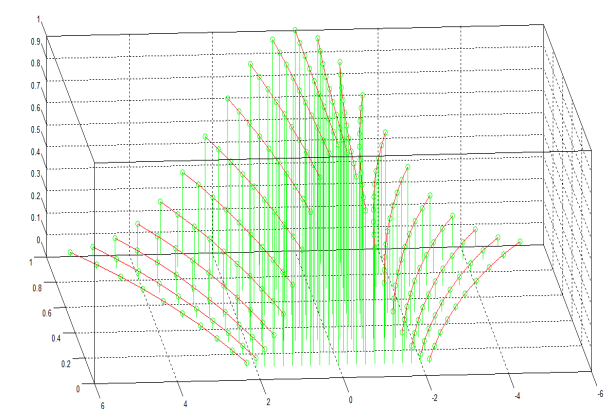

(a) Método previsor corretor de AdamsBashforth-Moulton

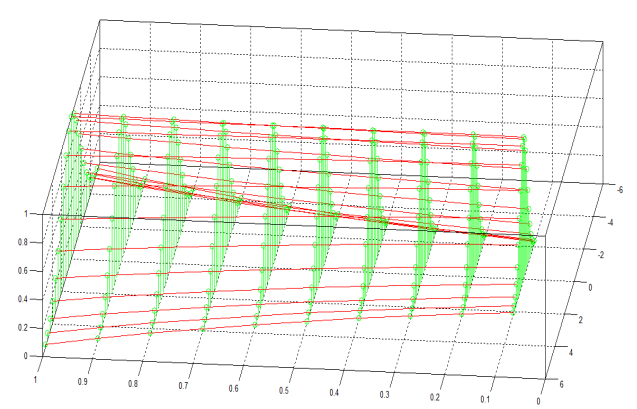

(b) Método previsor corretor de AdamsBashforth-Moulton

Figura 4.26: Curvas características com passo constante

$N(t)=(\cos (t), \sin (t),-1)$, usando o código desenvolvido, obtemos os seguintes resultados das Figuras 4.27(a), 4.27(b).

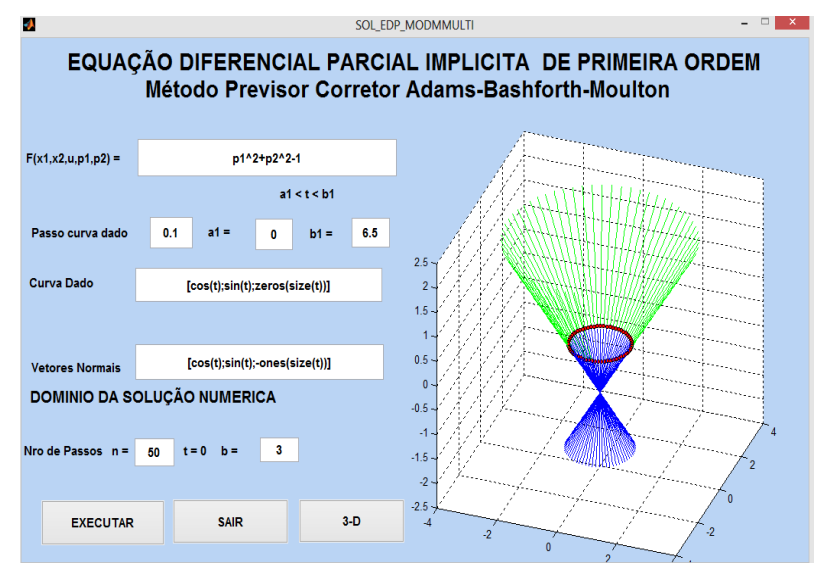

(a) Interface Adams-Bashforth-Moulton

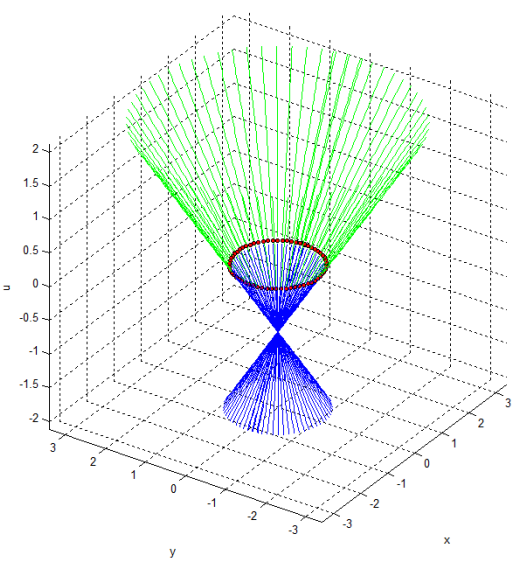

(b) Curvas características

Figura 4.27: Solução numérica da equação $\left(u_{x_{1}}\right)^{2}+\left(u_{x_{2}}\right)^{2}=1, \operatorname{com} x_{1}{ }^{2}+x_{2}{ }^{2}=1, u=0$, usando o Método de Adams-Bashforth-Moulton

Exemplo 4.13 Dada a EDP implícita $F\left(x_{1}, x_{2}, u, p_{1}, p_{2}\right)=x_{1}+x_{2}+u p_{1}+u p_{2}=0$, com a condição inicial $x_{1}^{2}+u^{2}=1$, que parametrizando fica como $\alpha(t)=(\cos (t), 0, \sin (t))$, com vetores normais dados por $N(t)=(-\cos (t) / \sin (t), 0,-1)$, usando o código desenvolvido, obtemos os resultados das Figuras 4.28(a), 4.28(b).

Exemplo 4.14 Dada a EDP implícita $F\left(x_{1}, x_{2}, u, p_{1}, p_{2}\right)=p_{1}{ }^{2}+p_{2}+u=0$, com a condição inicial $u\left(x_{1}, 0\right)=x_{1}$, que parametrizando fica como $\alpha(t)=(t, 0, t)$, com vetores normais dados por $N(t)=(1,-(1+t),-1)$, usando o código desenvolvido, obtemos os resultados das Figuras 4.29(a), 4.29(b). 


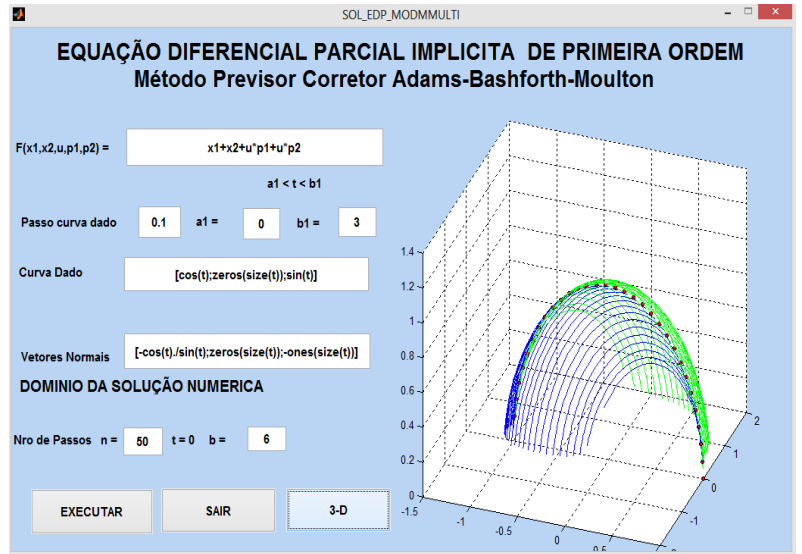

(a) Interface Adams-Bashforth-Moulton

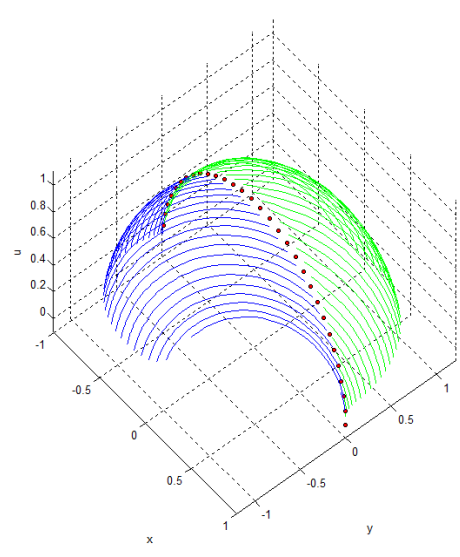

(b) Curvas características

Figura 4.28: Solução numérica da EDP $x_{1}+x_{2}+u p_{1}+u p_{2}=0$, com $x_{1}{ }^{2}+u^{2}=1$, usando o Método de Adams-Bashforth-Moulton

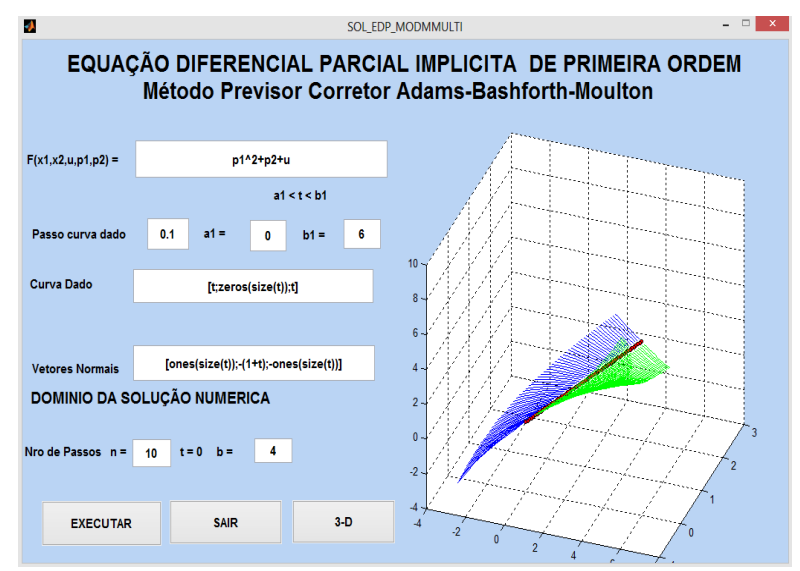

(a) Condições iniciais na interface

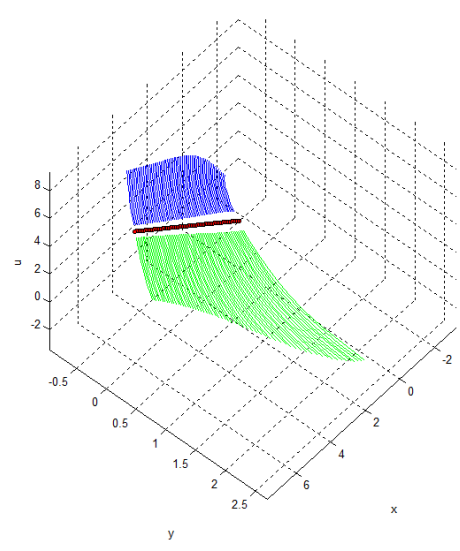

(b) Curvas características

Figura 4.29: Solução numérica da equação $F\left(x_{1}, x_{2}, u, p_{1}, p_{2}\right)=p_{1}{ }^{2}+p_{2}+u=0$, com condição inicial $u\left(x_{1}, 0\right)=x_{1}$, usando o Método de Adams-Bashforth-Moulton

\section{MÉTODO TIPO PROJEÇÃO}

A interface feita tem a opção para utilizar como método previsor o método de Euler e métodos de Runge-Kutta de segunda e quarta ordem. Na interface a escolha do método previsor segue de acordo com a numeração:

\section{Euler}

2. Runge-Kutta de segunda ordem

3. Runge-Kutta de quarta ordem

Exemplo 4.15 Seja a EDP implícita $F\left(x_{1}, x_{2}, u, p_{1}, p_{2}\right)=x_{1}+x_{2}+u p_{1}+u p_{2}=0$, com a condição inicial $x_{1}{ }^{2}+u^{2}=1$, que parametrizando fica como $\alpha(t)=(\cos (t), 0, \sin (t))$, com vetores 
normais dados por $N(t)=(-\cos (t) / \sin (t), 0,-1)$, usando o código desenvolvido, obtemos os resultados das Figuras 4.30(a), 4.30(b).

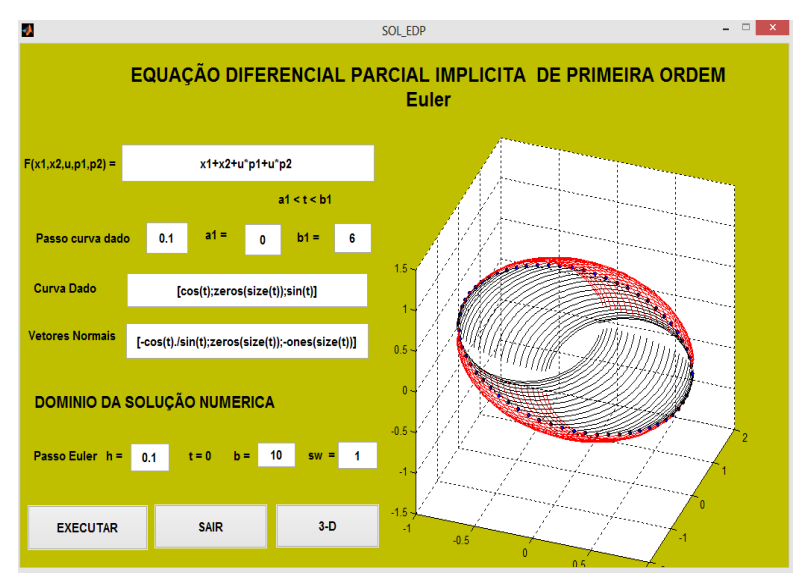

(a) Condições iniciais na interface

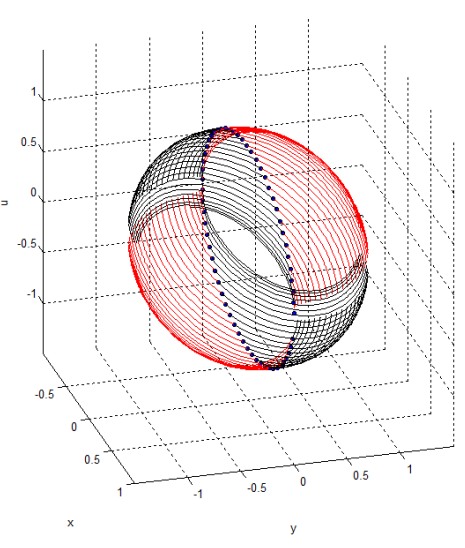

(b) Curvas características

Figura 4.30: Solução numérica da equação $F\left(x_{1}, x_{2}, u, p_{1}, p_{2}\right)=x_{1}+x_{2}+u p_{1}+u p_{2}=0$, com condição inicial $x_{1}{ }^{2}+u^{2}=1$, usando o Método de Euler

Exemplo 4.16 Dada a EDP implícita $F\left(x_{1}, x_{2}, u, p_{1}, p_{2}\right)=p_{1}{ }^{2}+p_{2}+u=0$, com a condição inicial $u\left(x_{1}, 0\right)=x_{1}$, que parametrizando fica como $\alpha(t)=(t, 0, t)$, com vetores normais dados por $N(t)=(1,-(1+t),-1)$, usando o código desenvolvido, obtemos os resultados das Figuras 4.31(a), 4.31(b).

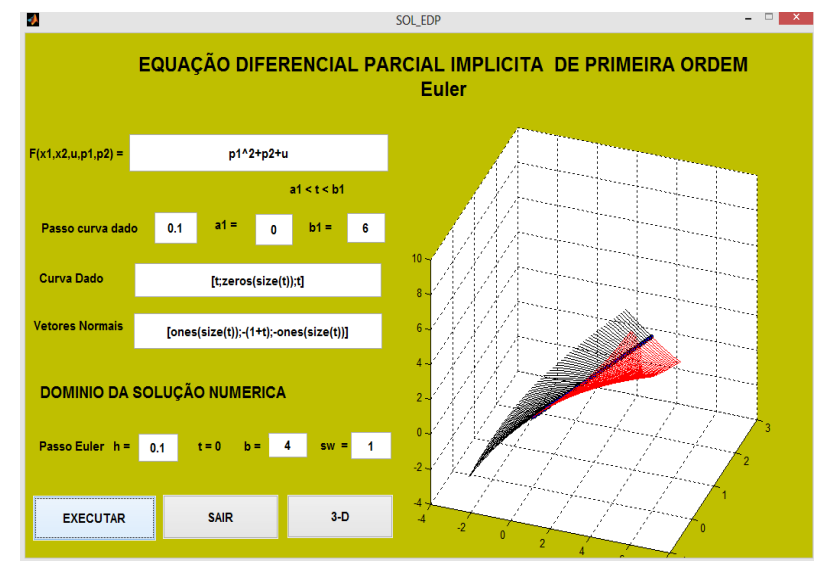

(a) Condições iniciais na interface

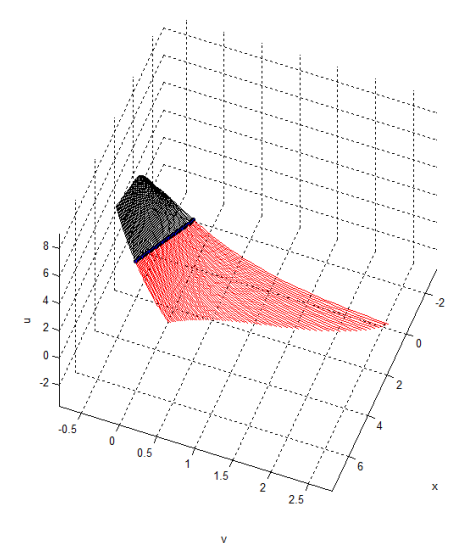

(b) Curvas características

Figura 4.31: Solução numérica da equação $F\left(x_{1}, x_{2}, u, p_{1}, p_{2}\right)=p_{1}{ }^{2}+p_{2}+u=0$, com condição inicial $u\left(x_{1}, 0\right)=x_{1}$, usando o Método de Euler

Agora utilizaremos a interface com método previsor diferente escolhido a opção 2 que é equivalente ao método de Runge-Kutta de segunda ordem. 
Exemplo 4.17 Seja a EDP implícita $F\left(x_{1}, x_{2}, u, p_{1}, p_{2}\right)=x_{1}+x_{2}+u p_{1}+u p_{2}=0$, com a condição inicial $x_{1}{ }^{2}+u^{2}=1$, que parametrizando fica como $\alpha(t)=(\cos (t), 0, \sin (t))$, com vetores normais dados por $N(t)=(-\cos (t) / \sin (t), 0,-1)$, usando o código desenvolvido, obtemos os resultados das Figuras 4.32(a), 4.32(b).

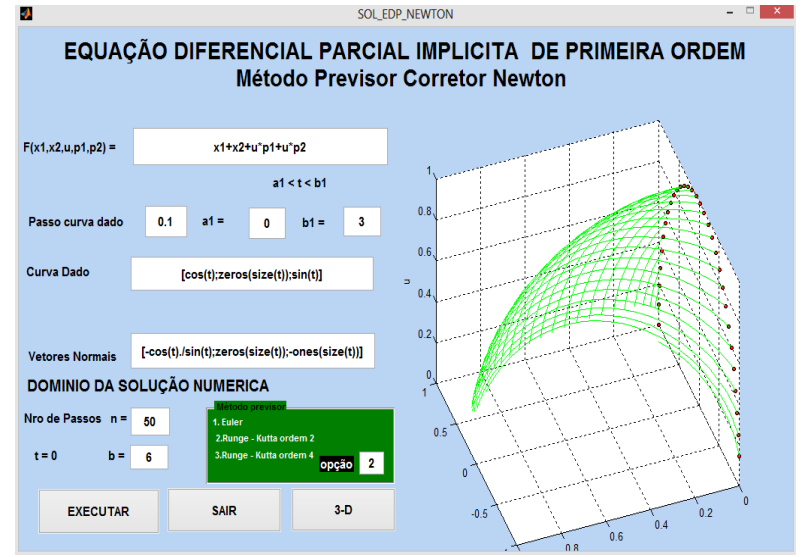

(a) Condições iniciais na interface

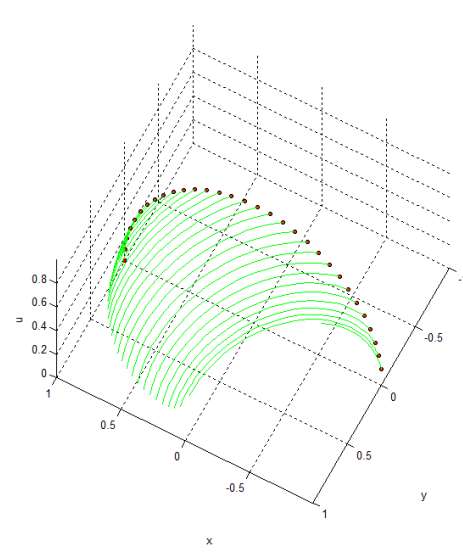

(b) Curvas características

Figura 4.32: Solução numérica da equação $F\left(x_{1}, x_{2}, u, p_{1}, p_{2}\right)=x_{1}+x_{2}+u p_{1}+u p_{2}=0$, com condição inicial $x_{1}^{2}+u^{2}=1$, para $\alpha(t)=(\cos (t), 0, \sin (t)), t \in[0,3]$, usando o Método de

Runge-Kutta de ordem 2

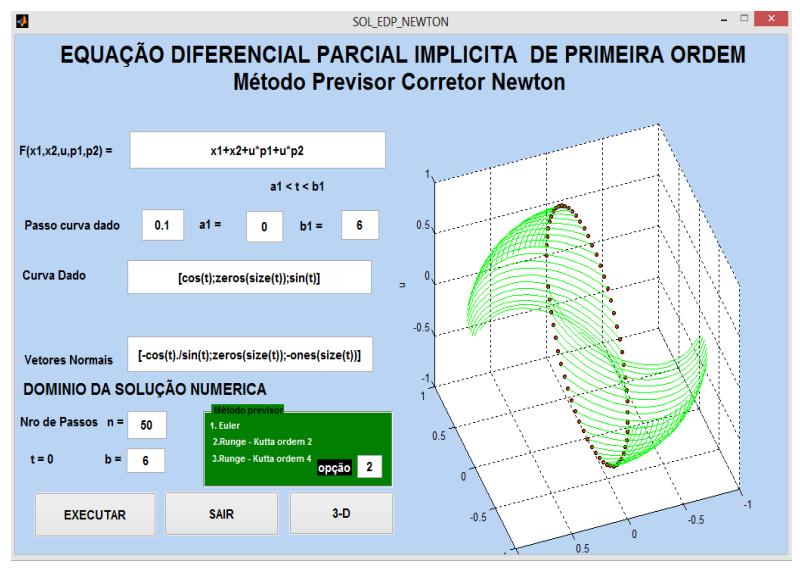

(a) Condições iniciais na interface

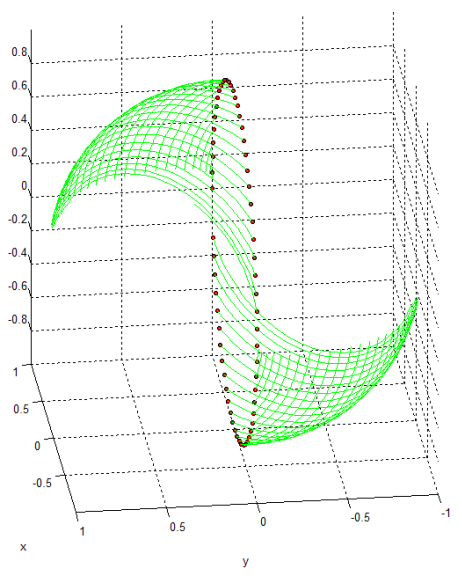

(b) Curvas características

Figura 4.33: Solução numérica da equação $F\left(x_{1}, x_{2}, u, p_{1}, p_{2}\right)=x_{1}+x_{2}+u p_{1}+u p_{2}=0$, com condição inicial $x_{1}{ }^{2}+u^{2}=1$, para $\alpha(t)=(\cos (t), 0, \sin (t)), t \in[0,6]$, usando o Método de Runge-Kutta de ordem 2 
Exemplo 4.18 Dada a EDP

$$
\begin{gathered}
\left(u_{x_{1}}\right)^{2}+\left(u_{x_{2}}\right)^{2}=1 \\
x_{1}{ }^{2}+x_{2}{ }^{2}=1, u=0
\end{gathered}
$$

a equação fica descrita por $F\left(x_{1}, x_{2}, u, p_{1}, p_{2}\right)=p_{1}{ }^{2}+p_{2}{ }^{2}-1=0$, ao parametrizar a curva inicial obtemos $\alpha(t)=(\cos (t), \sin (t), 0)$, que tem como vetor normal $N(t)=(\cos (t), \sin (t),-1)$

A solução analítica da equação é $u\left(x_{1}, x_{2}\right)=-1+\sqrt{x_{1}^{2}+x_{2}^{2}}$, as Tabelas 4.1, 4.2, 4.3, 4.4, e a Figura 4.34 permitem comparar o erro cometido ao usar os métodos de Adams-BashforthMulton(A.B.M.) e Runge-Kutta de quinta ordem(R.K.5) com o método do tipo Projeção. Consideraremos os seguintes dados

$n:$ Número de iterações

$h:$ Passo $h=\frac{b-t_{0}}{n}, \operatorname{com} b=3, t_{0}=0$

$\alpha$ : Curva dado definida como $\alpha:[0,3] \rightarrow \mathbf{R}^{3}, \alpha(t)=(\cos (t), \sin (t), 0)$

\begin{tabular}{|c|c|c|c|c|}
\hline$n$ & $h$ & Método de A.B.M & Método de R.K.5 & Método da Projeção \\
\hline 20 & 0.15 & $3.393112 \mathrm{e}-015$ & $3.664577 \mathrm{e}-015$ & $3.264438 \mathrm{e}-0.15$ \\
50 & 0.06 & $1.097954 \mathrm{e}-014$ & $2.511082 \mathrm{e}-014$ & $1.103252 \mathrm{e}-014$ \\
100 & 0.03 & $3.299531 \mathrm{e}-014$ & $3.770542 \mathrm{e}-014$ & $3.299972 \mathrm{e}-014$ \\
\hline
\end{tabular}

Tabela 4.1: Tabela de comparação do erro pelo número de iterações, ao resolver a equação $\left(u_{x_{1}}\right)^{2}+\left(u_{x_{2}}\right)^{2}=1, \operatorname{com} x_{1}{ }^{2}+x_{2}{ }^{2}=1, u=0$, Método de projeção de Euler-Newton

\begin{tabular}{|c|c|c|c|c|}
\hline$n$ & $h$ & Método de A.B.M & Método de RK5 & Método da Projeção \\
\hline 20 & 0.15 & $3.393112 \mathrm{e}-015$ & $3.664577 \mathrm{e}-015$ & $3.393112 \mathrm{e}-015$ \\
50 & 0.06 & $1.097954 \mathrm{e}-014$ & $2.511082 \mathrm{e}-014$ & $1.097954 \mathrm{e}-014$ \\
100 & 0.03 & $3.299531 \mathrm{e}-014$ & $3.770542 \mathrm{e}-014$ & $3.299531 \mathrm{e}-014$ \\
\hline
\end{tabular}

Tabela 4.2: Tabela de comparação do erro pelo número de iterações, ao resolver a equação $\left(u_{x_{1}}\right)^{2}+\left(u_{x_{2}}\right)^{2}=1, \operatorname{com} x_{1}^{2}+x_{2}^{2}=1, u=0$, Método de projeção R.K.4-Newton 
(a) Solução Método de Adams-Bashforth-Moulton

\begin{tabular}{|c|c|c|c|}
\hline Iteração & Solução aproximada & Soluçaô analitica & erro de truncamento \\
\hline$i=1$ & $\mathrm{~V}=-1.110223 \mathrm{e}-016$ & $\mathrm{u}=0.000000 \mathrm{e}+000$ & erro $=-1.110223 \mathrm{e}-016$ \\
\hline & $\mathrm{V}=2.121320 \mathrm{e}-001$ & $\mathrm{u}=2.121320 \mathrm{e}-001$ & erro $=5.551115 \mathrm{e}-017$ \\
\hline$i=3$ & $\mathrm{~V}=4.242641 \mathrm{e}-001$ & $\mathrm{u}=4.242641 \mathrm{e}-001$ & erro $=1.110223 \mathrm{e}-016$ \\
\hline & $\mathrm{V}=6.363961 \mathrm{e}-001$ & $\mathrm{u}=6.363961 \mathrm{e}-001$ & erro $=2.220446 \mathrm{e}-016$ \\
\hline$i=5$ & $\mathrm{~V}=8.485281 \mathrm{e}-001$ & $\mathrm{a}=8.485281 \mathrm{e}-001$ & erro $=2.220446 \mathrm{e}-016$ \\
\hline & $\mathrm{V}=1.060660 \mathrm{e}+000$ & $\mathrm{u}=1.060660 \mathrm{e}+000$ & erro $=2.220446 \mathrm{e}-016$ \\
\hline & $\mathrm{V}=1.272792 \mathrm{e}+000$ & $\mathrm{u}=1.272792 \mathrm{e}+000$ & erro $=2.220446 \mathrm{e}-016$ \\
\hline$i=8$ & $\mathrm{~V}=1.484924 \mathrm{e}+000$ & $\mathrm{u}=1.484924 \mathrm{e}+000$ & erro $=2.220446 \mathrm{e}-016$ \\
\hline$i=9$ & $\mathrm{U}=1.697056 \mathrm{e}+000$ & $\mathrm{u}=1.697056 \mathrm{e}+000$ & erro $=2.220446 \mathrm{e}-016$ \\
\hline & $\sigma=1.909188 \mathrm{e}+000$ & $\mathrm{u}=1.909188 \mathrm{e}+000$ & erro $=6.661338 \mathrm{e}-016$ \\
\hline & $\mathrm{V}=2.121320 \mathrm{e}+000$ & $\mathrm{u}=2.121320 \mathrm{e}+000$ & erro $=4.440892 \mathrm{e}-016$ \\
\hline $\mathrm{i}=12$ & $\mathrm{~V}=2.333452 \mathrm{e}+000$ & $\mathrm{u}=2.333452 \mathrm{e}+000$ & erro $=8.881784 \mathrm{e}-016$ \\
\hline$i=13$ & $\mathrm{~V}=2.545584 \mathrm{e}+000$ & $\mathrm{u}=2.545584 \mathrm{e}+000$ & erro $=8.881784 \mathrm{e}-016$ \\
\hline$i=14$ & $\mathrm{~V}=2.757716 \mathrm{e}+000$ & $\mathrm{u}=2.757716 \mathrm{e}+000$ & erro $=8.881784 \mathrm{e}-016$ \\
\hline & $\mathrm{U}=2.969848 \mathrm{e}+000$ & $\mathrm{u}=2.969848 \mathrm{e}+000$ & erro $=8.881784 \mathrm{e}-016$ \\
\hline$i=16$ & $\mathrm{~V}=3.181981 \mathrm{e}+000$ & $\mathrm{u}=3.181981 \mathrm{e}+000$ & erro $=8.881784 \mathrm{e}-016$ \\
\hline$i=17$ & $\mathrm{~V}=3.394113 \mathrm{e}+000$ & $\mathrm{u}=3.394113 \mathrm{e}+000$ & erro $=1.332268 \mathrm{e}-015$ \\
\hline$i=18$ & $\mathrm{~J}=3.606245 \mathrm{e}+000$ & $\mathrm{u}=3.606245 \mathrm{e}+000$ & erro $=8.881784 \mathrm{e}-016$ \\
\hline & $\mathrm{V}=3.818377 \mathrm{e}+000$ & $\mathrm{u}=3.818377 \mathrm{e}+000$ & erro $=4.440892 \mathrm{e}-016$ \\
\hline & $\sigma=4.030509 \mathrm{e}+000$ & $\mathrm{u}=4.030509 \mathrm{e}+000$ & erro $=8.881784 \mathrm{e}-016$ \\
\hline & $\mathrm{V}=4.242641 \mathrm{e}+000$ & $\mathrm{u}=4.242641 \mathrm{e}+000$ & erro $=1.776357 \mathrm{e}-015$ \\
\hline
\end{tabular}

Erro cometido $3.393112 \mathrm{e}-015$ (b) Solução Método de Projeção Euler-Newton

\begin{tabular}{|c|c|c|c|}
\hline \multicolumn{4}{|c|}{ Command Window } \\
\hline Iteração & Solução aproximada & Soluçaõ analitica & erro de truncamento \\
\hline$i=1$ & $\mathrm{~V}=-1.110223 \mathrm{e}-016$ & $\mathrm{u}=0.000000 \mathrm{e}+000$ & erro $=-1.110223 \mathrm{e}-016$ \\
\hline$i=2$ & $\mathrm{~J}=2.121320 \mathrm{e}-001$ & $\mathrm{u}=2.121320 \mathrm{e}-001$ & erro $=2.775558 \mathrm{e}-017$ \\
\hline & $\mathrm{U}=4.242641 \mathrm{e}-001$ & $\mathrm{u}=4.242641 \mathrm{e}-001$ & erro $=5.551115 \mathrm{e}-017$ \\
\hline$i=4$ & $\mathrm{U}=6.363961 \mathrm{e}-001$ & $\mathrm{u}=6.363961 \mathrm{e}-001$ & erro $=1.110223 \mathrm{e}-016$ \\
\hline$i=5$ & $\mathrm{~V}=8.485281 \mathrm{e}-001$ & $\mathrm{u}=8.485281 \mathrm{e}-001$ & erro $=3.330669 \mathrm{e}-016$ \\
\hline & $\mathrm{V}=1.060660 \mathrm{e}+000$ & $\mathrm{u}=1.060660 \mathrm{e}+000$ & erro $=2.220446 \mathrm{e}-016$ \\
\hline & $\mathrm{U}=1.272792 \mathrm{e}+000$ & $\mathrm{u}=1.272792 \mathrm{e}+000$ & erro $=2.220446 \mathrm{e}-016$ \\
\hline & $\mathrm{V}=1.484924 \mathrm{e}+000$ & $\mathrm{u}=1.484924 \mathrm{e}+000$ & erro $=2.220446 \mathrm{e}-016$ \\
\hline$i=9$ & $\mathrm{~V}=1.697056 \mathrm{e}+000$ & $\mathrm{u}=1.697056 \mathrm{e}+000$ & erro $=2.220446 \mathrm{e}-016$ \\
\hline$i=10$ & $\mathrm{~V}=1.909188 \mathrm{e}+000$ & $\mathrm{u}=1.909188 \mathrm{e}+000$ & erro $=6.661338 \mathrm{e}-016$ \\
\hline & $\mathrm{U}=2.121320 \mathrm{e}+000$ & $\mathrm{u}=2.121320 \mathrm{e}+000$ & erro $=4.440892 \mathrm{e}-016$ \\
\hline & $\mathrm{V}=2.333452 \mathrm{e}+000$ & $\mathrm{u}=2.333452 \mathrm{e}+000$ & erro $=8.881784 \mathrm{e}-016$ \\
\hline$i=13$ & $\mathrm{~V}=2.545584 \mathrm{e}+000$ & $\mathrm{u}=2.545584 \mathrm{e}+000$ & erro $=8.881784 \mathrm{e}-016$ \\
\hline$i=14$ & $\mathrm{~V}=2.757716 \mathrm{e}+000$ & $\mathrm{u}=2.757716 \mathrm{e}+000$ & erro $=8.881784 \mathrm{e}-016$ \\
\hline$i=15$ & $\mathrm{~V}=2.969848 \mathrm{e}+000$ & $\mathrm{u}=2.969848 \mathrm{e}+000$ & erro $=8.881784 \mathrm{e}-016$ \\
\hline & $\mathrm{U}=3.181981 \mathrm{e}+000$ & $\mathrm{u}=3.181981 \mathrm{e}+000$ & erro $=8.881784 \mathrm{e}-016$ \\
\hline$i=17$ & $\mathrm{~V}=3.394113 \mathrm{e}+000$ & $\mathrm{u}=3.394113 \mathrm{e}+000$ & erro $=1.332268 \mathrm{e}-015$ \\
\hline$i=18$ & $\mathrm{~V}=3.606245 \mathrm{e}+000$ & $\mathrm{u}=3.606245 \mathrm{e}+000$ & erro $=8.881784 e-016$ \\
\hline$i=19$ & $\mathrm{~V}=3.818377 \mathrm{e}+000$ & $\mathrm{u}=3.818377 \mathrm{e}+000$ & erro $=4.440892 \mathrm{e}-016$ \\
\hline$i=20$ & $\mathrm{~V}=4.030509 \mathrm{e}+000$ & $\mathrm{u}=4.030509 \mathrm{e}+000$ & erro $=8.881784 \mathrm{e}-016$ \\
\hline & $\mathrm{V}=4.242641 \mathrm{e}+000$ & $\mathrm{u}=4.242641 \mathrm{e}+000$ & erro $=1.776357 \mathrm{e}-015$ \\
\hline
\end{tabular}

Erro cometido 3.264438e-015

Tabela 4.3: Erro cometido ao resolver a equação $\left(u_{x_{1}}\right)^{2}+\left(u_{x_{2}}\right)^{2}=1, \operatorname{com} x_{1}{ }^{2}+x_{2}{ }^{2}=1, u=0$

(a) Solução Método de Runge-Kutta de ordem 5

\begin{tabular}{|c|c|c|c|}
\hline \multicolumn{4}{|c|}{ ommand Window } \\
\hline Iteração & Solução aproximada & Soluçaõ analitica & erro de truncamento \\
\hline & $\mathrm{V}=-1.110223 \mathrm{e}-016$ & $\mathrm{u}=0.000000 \mathrm{e}+000$ & erro $=-1.110223 \mathrm{e}-016$ \\
\hline$i=2$ & $\mathrm{U}=2.121320 \mathrm{e}-001$ & $\mathrm{u}=2.121320 \mathrm{e}-001$ & erro $=2.220446 \mathrm{e}-016$ \\
\hline$i=3$ & $\mathrm{~V}=4.242641 \mathrm{e}-001$ & $\mathrm{u}=4.242641 \mathrm{e}-001$ & erro $=4.440892 \mathrm{e}-016$ \\
\hline & $\mathrm{V}=6.363961 \mathrm{e}-001$ & $\mathrm{u}=6.363961 \mathrm{e}-001$ & erro $=7.771561 \mathrm{e}-016$ \\
\hline$i=5$ & $\mathrm{U}=8.485281 \mathrm{e}-001$ & $\mathrm{u}=8.485281 \mathrm{e}-001$ & erro $=1.332268 \mathrm{e}-015$ \\
\hline$i=6$ & $\mathrm{~V}=1.060660 \mathrm{e}+000$ & $\mathrm{u}=1.060660 \mathrm{e}+000$ & erro $=1.110223 \mathrm{e}-015$ \\
\hline$i=7$ & $\mathrm{U}=1.272792 \mathrm{e}+000$ & $\mathrm{u}=1.272792 \mathrm{e}+000$ & erro $=6.661338 \mathrm{e}-016$ \\
\hline$i=8$ & $\mathrm{~V}=1.484924 \mathrm{e}+000$ & $\mathrm{u}=1.484924 \mathrm{e}+000$ & erro $=2.220446 \mathrm{e}-016$ \\
\hline$i=9$ & $\mathrm{~V}=1.697056 \mathrm{e}+000$ & $\mathrm{u}=1.697056 \mathrm{e}+000$ & erro $=-2.220446 \mathrm{e}-016$ \\
\hline$i=10$ & $\mathrm{U}=1.909188 \mathrm{e}+000$ & $\mathrm{u}=1.909188 \mathrm{e}+000$ & erro $=-6.661338 \mathrm{e}-016$ \\
\hline$i=11$ & $\mathrm{U}=2.121320 \mathrm{e}+000$ & $\mathrm{u}=2.121320 \mathrm{e}+000$ & erro $=-4.440892 e-016$ \\
\hline & $\mathrm{U}=2.333452 \mathrm{e}+000$ & $\mathrm{u}=2.333452 \mathrm{e}+000$ & erro $=-8.881784 \mathrm{e}-016$ \\
\hline & $\mathrm{V}=2.545584 \mathrm{e}+000$ & $\mathrm{u}=2.545584 \mathrm{e}+000$ & erro $=-1.332268 \mathrm{e}-015$ \\
\hline$i=14$ & $\mathrm{~V}=2.757716 \mathrm{e}+000$ & $\mathrm{u}=2.757716 \mathrm{e}+000$ & erro $=-1.776357 \mathrm{e}-015$ \\
\hline$i=15$ & $\mathrm{~V}=2.969848 \mathrm{e}+000$ & $\mathrm{u}=2.969848 \mathrm{e}+000$ & erro $=-2.220446 \mathrm{e}-015$ \\
\hline & $\mathrm{U}=3.181981 \mathrm{e}+000$ & $\mathrm{u}=3.181981 \mathrm{e}+000$ & erro $=-2.664535 \mathrm{e}-015$ \\
\hline$i=17$ & $\mathrm{~V}=3.394113 \mathrm{e}+000$ & $\mathrm{u}=3.394113 \mathrm{e}+000$ & erro $=-2.220446 \mathrm{e}-015$ \\
\hline$i=18$ & $\mathrm{U}=3.606245 \mathrm{e}+000$ & $\mathrm{u}=3.606245 \mathrm{e}+000$ & erro $=-2.664535 \mathrm{e}-015$ \\
\hline & $\mathrm{V}=3.818377 \mathrm{e}+000$ & $\mathrm{u}=3.818377 \mathrm{e}+000$ & erro $=-2.220446 \mathrm{e}-015$ \\
\hline & $\mathrm{V}=4.030509 \mathrm{e}+000$ & $\mathrm{u}=4.030509 \mathrm{e}+000$ & erro $=-2.664535 \mathrm{e}-015$ \\
\hline & $\mathrm{U}=4.242641 \mathrm{e}+000$ & $\mathrm{u}=4.242641 \mathrm{e}+000$ & erro $=-1.776357 \mathrm{e}-015$ \\
\hline
\end{tabular}

Erro cometido $3.664577 \mathrm{e}-015$ (b) Solução Método de Projeção Euler-Newton

\begin{tabular}{|c|c|c|c|}
\hline \multicolumn{4}{|c|}{ ommand Window } \\
\hline Iteração & Solução aproximada & Soluçaõ analitica & erro de truncamento \\
\hline$i=1$ & $\mathrm{~V}=-1.110223 \mathrm{e}-016$ & $\mathrm{u}=0.000000 \mathrm{e}+000$ & erro $=-1.110223 \mathrm{e}-016$ \\
\hline$i=2$ & $\mathrm{~V}=2.121320 \mathrm{e}-001$ & $\mathrm{u}=2.121320 \mathrm{e}-001$ & erro $=2.775558 \mathrm{e}-017$ \\
\hline & $\mathrm{U}=4.242641 \mathrm{e}-001$ & $\mathrm{u}=4.242641 \mathrm{e}-001$ & erro $=5.551115 e-017$ \\
\hline & $\mathrm{V}=6.363961 \mathrm{e}-001$ & $\mathrm{u}=6.363961 \mathrm{e}-001$ & erro $=1.110223 \mathrm{e}-016$ \\
\hline$i=5$ & $\mathrm{U}=8.485281 \mathrm{e}-001$ & $\mathrm{u}=8.485281 \mathrm{e}-001$ & erro $=3.330669 \mathrm{e}-016$ \\
\hline$i=6$ & $\mathrm{~V}=1.060660 \mathrm{e}+000$ & $u=1.060660 e+000$ & erro $=2.220446 e-016$ \\
\hline & $\mathrm{V}=1.272792 \mathrm{e}+000$ & $u=1.272792 \mathrm{e}+000$ & erro $=2.220446 \mathrm{e}-016$ \\
\hline & $\mathrm{V}=1.484924 \mathrm{e}+000$ & $\mathrm{u}=1.484924 \mathrm{e}+000$ & erro $=2.220446 \mathrm{e}-016$ \\
\hline$i=9$ & $\mathrm{~V}=1.697056 \mathrm{e}+000$ & $\mathrm{u}=1.697056 \mathrm{e}+000$ & erro $=2.220446 \mathrm{e}-016$ \\
\hline$i=10$ & $\mathrm{~V}=1.909188 \mathrm{e}+000$ & $\mathrm{u}=1.909188 \mathrm{e}+000$ & erro $=6.661338 \mathrm{e}-016$ \\
\hline & $\mathrm{V}=2.121320 \mathrm{e}+000$ & $\mathrm{u}=2.121320 \mathrm{e}+000$ & erro $=4.440892 \mathrm{e}-016$ \\
\hline & $\mathrm{U}=2.333452 \mathrm{e}+000$ & $\mathrm{u}=2.333452 \mathrm{e}+000$ & erro $=8.881784 \mathrm{e}-016$ \\
\hline & $\mathrm{V}=2.545584 \mathrm{e}+000$ & $\mathrm{u}=2.545584 \mathrm{e}+000$ & erro $=8.881784 \mathrm{e}-016$ \\
\hline & $\mathrm{V}=2.757716 \mathrm{e}+000$ & $\mathrm{u}=2.757716 \mathrm{e}+000$ & erro $=8.881784 \mathrm{e}-016$ \\
\hline & $\mathrm{V}=2.969848 \mathrm{e}+000$ & $\mathrm{u}=2.969848 \mathrm{e}+000$ & erro $=8.881784 \mathrm{e}-016$ \\
\hline & $\mathrm{U}=3.181981 \mathrm{e}+000$ & $\mathrm{u}=3.181981 \mathrm{e}+000$ & erro $=8.881784 \mathrm{e}-016$ \\
\hline$i=17$ & $\mathrm{~V}=3.394113 \mathrm{e}+000$ & $\mathrm{u}=3.394113 \mathrm{e}+000$ & erro $=1.332268 \mathrm{e}-015$ \\
\hline$i=18$ & $\mathrm{~V}=3.606245 \mathrm{e}+000$ & $\mathrm{u}=3.606245 \mathrm{e}+000$ & erro $=8.881784 \mathrm{e}-016$ \\
\hline & $\mathrm{V}=3.818377 \mathrm{e}+000$ & $\mathrm{u}=3.818377 \mathrm{e}+000$ & erro $=4.440892 \mathrm{e}-016$ \\
\hline & $\mathrm{V}=4.030509 \mathrm{e}+000$ & $\mathrm{u}=4.030509 \mathrm{e}+000$ & erro $=8.881784 \mathrm{e}-016$ \\
\hline $\begin{array}{l}1=20 \\
i=21\end{array}$ & $\mathrm{~V}=4.242641 \mathrm{e}+000$ & $\mathrm{u}=4.242641 \mathrm{e}+000$ & 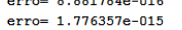 \\
\hline
\end{tabular}

Erro cometido 3.264438e-015

Tabela 4.4: Erro cometido ao resolver a equação $\left(u_{x_{1}}\right)^{2}+\left(u_{x_{2}}\right)^{2}=1, \operatorname{com} x_{1}{ }^{2}+x_{2}{ }^{2}=1, u=0$

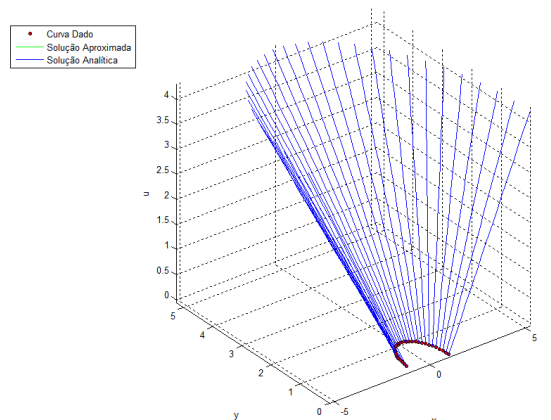

Figura 4.34: Solução numérica da equação $\left(u_{x_{1}}\right)^{2}+\left(u_{x_{2}}\right)^{2}=1, \operatorname{com} x_{1}{ }^{2}+x_{2}{ }^{2}=1, u=0$, usando o Método tipo Projeção de Euler-Newton 
Exemplo 4.19 Dada a EDP

$$
\begin{gathered}
u_{x_{1}} u_{x_{2}}=u \\
u\left(0, x_{2}\right)=x_{2}^{2}
\end{gathered}
$$

a equação fica descrita por $F\left(x_{1}, x_{2}, u, p_{1}, p_{2}\right)=p_{1} p_{2}-u=0$ ao parametrizar a curva inicial obtemos $\alpha(t)=\left(0, t, t^{2}\right)$, que tem como vetor normal $N(t)=(t / 2,2 t,-1)$

A solução analítica da equação é $u\left(x_{1}, x_{2}\right)=\left(x_{1} / 4+x_{2}\right)^{2}$, as Tabelas 4.5, 4.6, 4.7, 4.8, e a Figura 4.35 permitem comparar o erro cometido ao usar os métodos de Adams-BashforthMulton(A.B.M.) e Runge-Kutta de quinta ordem(R.K.5) com o método do tipo Projeção. Consideraremos os seguintes dados

$n:$ Número de iterações

$h:$ Passo $h=\frac{b-t_{0}}{n}, \operatorname{com} b=3, t_{0}=0.1$

$\alpha$ : Curva dado definida como $\alpha:[0.1,3] \rightarrow \mathbf{R}^{3}, \alpha(t)=\left(0, t, t^{2}\right)$

\begin{tabular}{|c|c|c|c|c|}
\hline$n$ & $h$ & Método de A.B.M & Método de R.K.5 & Método da Projeção \\
\hline 20 & 0.145 & $9.959860 \mathrm{e}-001$ & $4.583976 \mathrm{e}-001$ & $5.467156 \mathrm{e}-001$ \\
50 & 0.058 & $7.220523 \mathrm{e}-001$ & $2.692534 \mathrm{e}-001$ & $3.226722 \mathrm{e}-001$ \\
100 & 0.029 & $5.353669 \mathrm{e}-001$ & $1.886051 \mathrm{e}-001$ & $2.238795 \mathrm{e}-001$ \\
\hline
\end{tabular}

Tabela 4.5: Tabela de comparação do erro pelo número de iterações, ao resolver a equação $u_{x_{1}} u_{x_{2}}=u, \operatorname{com} u\left(0, x_{2}\right)=x_{2}^{2}$, Método de projeção de Euler-Newton

\begin{tabular}{|c|c|c|c|c|}
\hline$n$ & $h$ & Método de A.B.M & Método de RK5 & Método da Projeção \\
\hline 20 & 0.145 & $9.959860 \mathrm{e}-001$ & $4.583976 \mathrm{e}-001$ & $3.434290 \mathrm{e}-001$ \\
50 & 0.058 & $7.220523 \mathrm{e}-001$ & $2.692534 \mathrm{e}-001$ & $2.094777 \mathrm{e}-001$ \\
100 & 0.029 & $5.353669 \mathrm{e}-001$ & $1.886051 \mathrm{e}-001$ & $1.472323 \mathrm{e}-001$ \\
\hline
\end{tabular}

Tabela 4.6: Tabela de comparação do erro pelo número de iterações, ao resolver a equação $u_{x_{1}} u_{x_{2}}=u$, com $u\left(0, x_{2}\right)=x_{2}^{2}$, Método de projeção R.K.4-Newton

Ao comparar os resultados das tabelas verificamos que o erro cometido ao usar o método da projeção é menor que o erro cometido usando os outros métodos. Por exemplo para $n=100$ subdivisões o passo correspondente é de $h=0.029$, obtendo-se um erro de $E=1.472323 e-001$ para o Método da Projeção, que é menor que o erro obtido pelo Método de Runge-Kutta de quinta ordem, $E=1.886051 e-001$ e menor também que o erro obtido pelo Método de Adams Bashforth Moulton $E=5.353669 e-001$. Sendo $E$ a diferença entre os valores aproximados da superfície e os valores da solução analítica para os pontos da malha calculada. 
(a) Solução Método de Adams-Bashforth-Moulton

\begin{tabular}{|c|c|c|c|}
\hline \multicolumn{4}{|c|}{ Command Window } \\
\hline Iteração & Solução aproximada & Soluçaõ analitica & erro de truncamento \\
\hline & $\mathrm{V}=9.000000 \mathrm{e}+000$ & $\mathrm{u}=9.000000 \mathrm{e}+000$ & erro $=0.000000 e+000$ \\
\hline$i=2$ & $V=9.269135 e+000$ & $u=9.270458 \mathrm{e}+000$ & erro $=-1.322414 \mathrm{e}-003$ \\
\hline & $\mathrm{V}=9.539024 \mathrm{e}+000$ & $\mathrm{u}=9.541638 \mathrm{e}+000$ & erro $=-2.614108 \mathrm{e}-003$ \\
\hline & $\mathrm{V}=9.809632 \mathrm{e}+000$ & $u=9.813508 \mathrm{e}+000$ & erro $=-3.876402 \mathrm{e}-003$ \\
\hline & $\mathrm{V}=1.008680 \mathrm{e}+001$ & $u=1.008477 e+001$ & erro $=2.035601 \mathrm{e}-003$ \\
\hline & $\mathrm{V}=1.036456 \mathrm{e}+001$ & $u=1.035673 e+001$ & erro $=7.827772 \mathrm{e}-003$ \\
\hline$i=7$ & $\mathrm{~V}=1.064287 \mathrm{e}+001$ & $\mathrm{u}=1.062935 \mathrm{e}+001$ & erro $=1.352183 \mathrm{e}-002$ \\
\hline$i=8$ & $\mathrm{~V}=1.092172 \mathrm{e}+001$ & $\mathrm{u}=1.090261 \mathrm{e}+001$ & erro $=1.911786 \mathrm{e}-002$ \\
\hline$i=9$ & $\mathrm{~V}=1.120106 \mathrm{e}+001$ & $u=1.117647 e+001$ & erro $=2.458829 \mathrm{e}-002$ \\
\hline$i=10$ & $\mathrm{~V}=1.148087 \mathrm{e}+001$ & $\mathrm{u}=1.145093 \mathrm{e}+001$ & erro $=2.993838 \mathrm{e}-002$ \\
\hline$i=11$ & $\mathrm{~V}=1.176112 \mathrm{e}+001$ & $\mathrm{u}=1.172594 \mathrm{e}+001$ & erro $=3.517294 \mathrm{e}-002$ \\
\hline$i=12$ & $\mathrm{~V}=1.204179 \mathrm{e}+001$ & $\mathrm{u}=1.200150 \mathrm{e}+001$ & erro $=4.029653 \mathrm{e}-002$ \\
\hline$i=13$ & $\mathrm{~V}=1.232288 \mathrm{e}+001$ & $\mathrm{u}=1.227756 \mathrm{e}+001$ & erro $=4.531358 \mathrm{e}-002$ \\
\hline$i=14$ & $\mathrm{~V}=1.260436 \mathrm{e}+001$ & $\mathrm{u}=1.255413 \mathrm{e}+001$ & erro $=5.022827 \mathrm{e}-002$ \\
\hline$i=15$ & $\mathrm{~V}=1.288621 \mathrm{e}+001$ & $\mathrm{u}=1.283117 \mathrm{e}+001$ & erro $=5.504455 \mathrm{e}-002$ \\
\hline$i=16$ & $\mathrm{~V}=1.316843 \mathrm{e}+001$ & $\mathrm{u}=1.310866 \mathrm{e}+001$ & erro $=5.976614 \mathrm{e}-002$ \\
\hline$i=17$ & $\mathrm{~V}=1.345100 \mathrm{e}+001$ & $\mathrm{u}=1.338660 \mathrm{e}+001$ & erro $=6.439656 \mathrm{e}-002$ \\
\hline$i=18$ & $\mathrm{~V}=1.373390 \mathrm{e}+001$ & $u=1.366496 \mathrm{e}+001$ & erro $=6.893916 \mathrm{e}-002$ \\
\hline & $\mathrm{V}=1.401713 \mathrm{e}+001$ & $\mathrm{u}=1.394373 \mathrm{e}+001$ & erro $=7.339710 \mathrm{e}-002$ \\
\hline & $\mathrm{V}=1.430066 \mathrm{e}+001$ & $\mathrm{u}=1.422289 \mathrm{e}+001$ & erro $=7.777337 \mathrm{e}-002$ \\
\hline 21 & $\mathrm{~V}=1.458450 \mathrm{e}+001$ & $\mathrm{u}=1.450243 \mathrm{e}+001$ & erro $=8.207082 \mathrm{e}-002$ \\
\hline
\end{tabular}

Erro cometido 9.959860 e- 001 (b) Solução Método de Projeção Euler-Newton

\begin{tabular}{|c|c|c|c|}
\hline \multicolumn{4}{|c|}{ Command Window } \\
\hline Iteração & Solução aproximada & Soluçaõ analitica & erro de truncamento \\
\hline & $\mathrm{V}=9.000000 \mathrm{e}+000$ & $\mathrm{u}=9.000000 \mathrm{e}+000$ & erro $=0.000000 \mathrm{e}+000$ \\
\hline & $\mathrm{V}=9.271854 \mathrm{e}+000$ & $\mathrm{u}=9.269881 \mathrm{e}+000$ & erro $=1.972417 \mathrm{e}-003$ \\
\hline$i=3$ & $\mathrm{~V}=9.544438 \mathrm{e}+000$ & $\mathrm{u}=9.540510 \mathrm{e}+000$ & erro $=3.928205 e-003$ \\
\hline & $\mathrm{U}=9.817718 \mathrm{e}+000$ & $\mathrm{u}=9.811850 \mathrm{e}+000$ & erro $=5.867560 \mathrm{e}-003$ \\
\hline$i=5$ & $\mathrm{~V}=1.009166 \mathrm{e}+001$ & $\mathrm{u}=1.008387 \mathrm{e}+001$ & erro $=7.790692 \mathrm{e}-003$ \\
\hline & $\mathrm{V}=1.036624 \mathrm{e}+001$ & $\mathrm{u}=1.035654 \mathrm{e}+001$ & erro $=9.697825 e-003$ \\
\hline & $\mathrm{V}=1.064142 \mathrm{e}+001$ & $\mathrm{u}=1.062984 \mathrm{e}+001$ & erro $=1.158919 \mathrm{e}-002$ \\
\hline & $\mathrm{V}=1.091719 \mathrm{e}+001$ & $\mathrm{u}=1.090372 \mathrm{e}+001$ & erro $=1.346503 \mathrm{e}-002$ \\
\hline & $\mathrm{V}=1.119351 \mathrm{e}+001$ & $\mathrm{u}=1.117818 \mathrm{e}+001$ & erro $=1.532558 \mathrm{e}-002$ \\
\hline & $\mathrm{V}=1.147036 \mathrm{e}+001$ & $\mathrm{u}=1.145319 \mathrm{e}+001$ & erro $=1.717109 \mathrm{e}-002$ \\
\hline & $\mathrm{V}=1.174773 \mathrm{e}+001$ & $\mathrm{u}=1.172872 \mathrm{e}+001$ & erro $=1.900181 \mathrm{e}-002$ \\
\hline & $\mathrm{V}=1.202558 \mathrm{e}+001$ & $\mathrm{u}=1.200476 \mathrm{e}+001$ & erro $=2.081799 \mathrm{e}-002$ \\
\hline & $\mathrm{V}=1.230390 \mathrm{e}+001$ & $\mathrm{u}=1.228128 \mathrm{e}+001$ & erro $=2.261987 \mathrm{e}-002$ \\
\hline & $\mathrm{V}=1.258268 \mathrm{e}+001$ & $\mathrm{u}=1.255827 \mathrm{e}+001$ & erro $=2.440771 \mathrm{e}-002$ \\
\hline$i=15$ & $\mathrm{~V}=1.286189 \mathrm{e}+001$ & $\mathrm{u}=1.283571 \mathrm{e}+001$ & erro $=2.618174 \mathrm{e}-002$ \\
\hline$i=16$ & $\mathrm{~V}=1.314152 \mathrm{e}+001$ & $\mathrm{u}=1.311358 \mathrm{e}+001$ & erro $=2.794222 \mathrm{e}-002$ \\
\hline$i=17$ & $\mathrm{~V}=1.342156 \mathrm{e}+001$ & $\mathrm{u}=1.339187 \mathrm{e}+001$ & erro $=2.968937 \mathrm{e}-002$ \\
\hline $1=18$ & $\mathrm{~V}=1.370198 \mathrm{e}+001$ & $\mathrm{u}=1.367056 \mathrm{e}+001$ & erro $=3.142343 \mathrm{e}-002$ \\
\hline$i=19$ & $\mathrm{~V}=1.398278 \mathrm{e}+001$ & $\mathrm{u}=1.394964 \mathrm{e}+001$ & erro $=3.314464 \mathrm{e}-002$ \\
\hline$i=20$ & $\mathrm{~V}=1.426394 \mathrm{e}+001$ & $\mathrm{u}=1.422909 \mathrm{e}+001$ & erro $=3.485323 \mathrm{e}-002$ \\
\hline & $\mathrm{V}=1.454545 \mathrm{e}+001$ & $\mathrm{u}=1.450890 \mathrm{e}+001$ & erro $=3.654941 \mathrm{e}-002$ \\
\hline
\end{tabular}

Brro cometido 5.467156e-001

Tabela 4.7: Erro cometido ao resolver a equação $u_{x_{1}} u_{x_{2}}=u, \operatorname{com} u\left(0, x_{2}\right)=x_{2}{ }^{2}$

(a) Solução Método de Runge-Kutta de ordem 5

\begin{tabular}{|c|c|c|c|}
\hline \multicolumn{4}{|l|}{ mand Wi } \\
\hline Iteração & Solução aproximada & Soluçaô analitica & erro de truncamento \\
\hline & $\mathrm{V}=9.000000 \mathrm{e}+000$ & $\mathrm{u}=9.000000 \mathrm{e}+000$ & erro $=0.000000 e+000$ \\
\hline$i=2$ & $\mathrm{~V}=9.271854 \mathrm{e}+000$ & $\mathrm{u}=9.269831 \mathrm{e}+000$ & erro $=2.022472 \mathrm{e}-003$ \\
\hline & $\mathrm{V}=9.544418 \mathrm{e}+000$ & $u=9.540422 e+000$ & erro $=3.996701 \mathrm{e}-003$ \\
\hline & $\mathrm{V}=9.817660 \mathrm{e}+000$ & $\mathrm{u}=9.811735 \mathrm{e}+000$ & erro $=5.924818 \mathrm{e}-003$ \\
\hline$i=5$ & $\mathrm{~V}=1.009155 \mathrm{e}+001$ & $\mathrm{u}=1.008374 \mathrm{e}+001$ & erro $=7.808820 \mathrm{e}-003$ \\
\hline$i=6$ & $\sigma=1.036605 \mathrm{e}+001$ & $\mathrm{u}=1.035640 \mathrm{e}+001$ & erro $=9.650584 \mathrm{e}-003$ \\
\hline$i=7$ & $\mathrm{~V}=1.064115 \mathrm{e}+001$ & $\mathrm{u}=1.062969 \mathrm{e}+001$ & erro $=1.145187 \mathrm{e}-002$ \\
\hline$i=8$ & $\mathrm{~V}=1.091680 \mathrm{e}+001$ & $\mathrm{u}=1.090359 \mathrm{e}+001$ & erro $=1.321435 \mathrm{e}-002$ \\
\hline$i=9$ & $\mathrm{~V}=1.119300 \mathrm{e}+001$ & $\mathrm{u}=1.117806 \mathrm{e}+001$ & erro $=1.493957 \mathrm{e}-002$ \\
\hline$i=10$ & $\mathrm{~V}=1.146972 \mathrm{e}+001$ & $\mathrm{u}=1.145309 \mathrm{e}+001$ & erro $=1.662901 \mathrm{e}-002$ \\
\hline$i=11$ & $\mathrm{~V}=1.174693 \mathrm{e}+001$ & $\mathrm{u}=1.172865 \mathrm{e}+001$ & erro $=1.828407 \mathrm{e}-002$ \\
\hline & $\mathrm{V}=1.202462 \mathrm{e}+001$ & $\mathrm{u}=1.200472 \mathrm{e}+001$ & erro $=1.990605 \mathrm{e}-002$ \\
\hline & $\mathrm{U}=1.230277 \mathrm{e}+001$ & $\mathrm{u}=1.228127 \mathrm{e}+001$ & erro $=2.149619 \mathrm{e}-002$ \\
\hline & $\mathrm{J}=1.258136 \mathrm{e}+001$ & $\mathrm{u}=1.255830 \mathrm{e}+001$ & erro $=2.305569 \mathrm{e}-002$ \\
\hline & $\mathrm{U}=1.286037 \mathrm{e}+001$ & $\mathrm{u}=1.283578 \mathrm{e}+001$ & erro $=2.458564 \mathrm{e}-002$ \\
\hline & $\mathrm{U}=1.313978 \mathrm{e}+001$ & $\mathrm{u}=1.311370 \mathrm{e}+001$ & erro $=2.608710 \mathrm{e}-002$ \\
\hline & $\mathrm{U}=1.341959 \mathrm{e}+001$ & $\mathrm{u}=1.339203 \mathrm{e}+001$ & erro $=2.756108 \mathrm{e}-002$ \\
\hline & $\mathrm{V}=1.369978 \mathrm{e}+001$ & $\mathrm{u}=1.367077 \mathrm{e}+001$ & erro $=2.900853 \mathrm{e}-002$ \\
\hline & $\mathrm{V}=1.398034 \mathrm{e}+001$ & $\mathrm{u}=1.394991 \mathrm{e}+001$ & erro $=3.043035 \mathrm{e}-002$ \\
\hline & $\mathrm{U}=1.426124 \mathrm{e}+001$ & $\mathrm{u}=1.422942 \mathrm{e}+001$ & erro $=3.182740 \mathrm{e}-002$ \\
\hline$i=21$ & $\mathrm{~V}=1.454249 \mathrm{e}+001$ & $\mathrm{u}=1.450929 \mathrm{e}+001$ & erro $=3.320051 \mathrm{e}-002$ \\
\hline
\end{tabular}

Brro cometido $4.583976 \mathrm{e}-001$ (b) Solução Método de Projeção Euler-Newton

\begin{tabular}{|c|c|c|c|}
\hline \multicolumn{4}{|c|}{ Command Window } \\
\hline Iteração & Solução aproximada & Soluçaô analitica & erro de truncamento \\
\hline & $\mathrm{V}=9.000000 \mathrm{e}+000$ & $\mathrm{u}=9.000000 \mathrm{e}+000$ & erro $=0.000000 e+000$ \\
\hline & $\mathrm{V}=9.271854 \mathrm{e}+000$ & $\mathrm{u}=9.269881 \mathrm{e}+000$ & erro $=1.972417 \mathrm{e}-003$ \\
\hline & $\mathrm{V}=9.544438 \mathrm{e}+000$ & $\mathrm{u}=9.540510 \mathrm{e}+000$ & erro $=3.928205 \mathrm{e}-003$ \\
\hline & $\mathrm{V}=9.817718 \mathrm{e}+000$ & $\mathrm{u}=9.811850 \mathrm{e}+000$ & erro $=5.867560 \mathrm{e}-003$ \\
\hline$i=5$ & $\mathrm{~V}=1.009166 \mathrm{e}+001$ & $u=1.008387 e+001$ & erro $=7.790692 \mathrm{e}-003$ \\
\hline & $\mathrm{V}=1.036624 \mathrm{e}+001$ & $\mathrm{u}=1.035654 \mathrm{e}+001$ & erro $=9.697825 e-003$ \\
\hline & $\mathrm{V}=1.064142 \mathrm{e}+001$ & $\mathrm{u}=1.062984 \mathrm{e}+001$ & erro $=1.158919 \mathrm{e}-002$ \\
\hline & $\mathrm{V}=1.091719 \mathrm{e}+001$ & $\mathrm{u}=1.090372 \mathrm{e}+001$ & erro $=1.346503 e-002$ \\
\hline & $\mathrm{V}=1.119351 \mathrm{e}+001$ & $\mathrm{u}=1.117818 \mathrm{e}+001$ & erro $=1.532558 \mathrm{e}-002$ \\
\hline$i=10$ & $\mathrm{~V}=1.147036 \mathrm{e}+001$ & $\mathrm{u}=1.145319 \mathrm{e}+001$ & erro $=1.717109 \mathrm{e}-002$ \\
\hline & $\mathrm{V}=1.174773 \mathrm{e}+001$ & $\mathrm{u}=1.172872 \mathrm{e}+001$ & erro $=1.900181 \mathrm{e}-002$ \\
\hline & $\mathrm{V}=1.202558 \mathrm{e}+001$ & $\mathrm{u}=1.200476 \mathrm{e}+001$ & erro $=2.081799 \mathrm{e}-002$ \\
\hline & $\mathrm{V}=1.230390 \mathrm{e}+001$ & $\mathrm{u}=1.228128 \mathrm{e}+001$ & erro $=2.261987 \mathrm{e}-002$ \\
\hline & $\mathrm{V}=1.258268 \mathrm{e}+001$ & $\mathrm{u}=1.255827 \mathrm{e}+001$ & erro $=2.440771 \mathrm{e}-002$ \\
\hline & $\mathrm{V}=1.286189 \mathrm{e}+001$ & $\mathrm{u}=1.283571 \mathrm{e}+001$ & erro $=2.618174 \mathrm{e}-002$ \\
\hline & $\mathrm{V}=1.314152 \mathrm{e}+001$ & $\mathrm{u}=1.311358 \mathrm{e}+001$ & erro $=2.794222 \mathrm{e}-002$ \\
\hline & $\mathrm{V}=1.342156 \mathrm{e}+001$ & $\mathrm{u}=1.339187 \mathrm{e}+001$ & erro $=2.968937 \mathrm{e}-002$ \\
\hline & $\mathrm{V}=1.370198 \mathrm{e}+001$ & $\mathrm{u}=1.367056 \mathrm{e}+001$ & erro $=3.142343 \mathrm{e}-002$ \\
\hline & $\mathrm{V}=1.398278 \mathrm{e}+001$ & $\mathrm{u}=1.394964 \mathrm{e}+001$ & erro $=3.314464 \mathrm{e}-002$ \\
\hline & $\mathrm{V}=1.426394 \mathrm{e}+001$ & $\mathrm{u}=1.422909 \mathrm{e}+001$ & erro $=3.485323 \mathrm{e}-002$ \\
\hline & $\mathrm{V}=1.454545 \mathrm{e}+001$ & $\mathrm{u}=1.450890 \mathrm{e}+001$ & erro $=3.654941 \mathrm{e}-002$ \\
\hline
\end{tabular}

Erro cometido 5.467156e-001

Tabela 4.8: Erro cometido ao resolver a equação $u_{x_{1}} u_{x_{2}}=u, \operatorname{com} u\left(0, x_{2}\right)=x_{2}{ }^{2}$

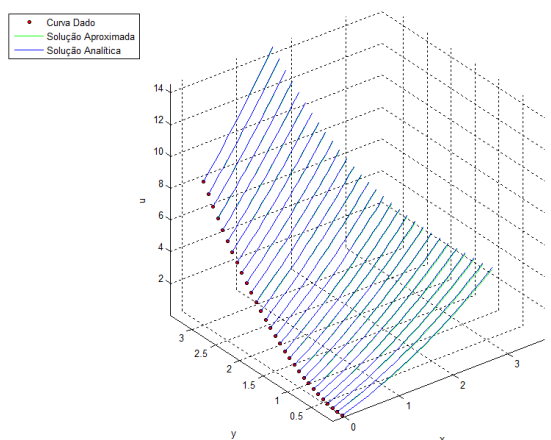

Figura 4.35: Solução numérica da equação $u_{x_{1}} u_{x_{2}}=u$, com $u\left(0, x_{2}\right)=x_{2}{ }^{2}$, usando o Método tipo Projeção de Euler-Newton 


\subsection{Equação de Burgers}

Dentro dos diferentes modelos de EDPs temos o fenômeno de ondas, determinado por equações tais como

$$
\begin{array}{r}
u_{y}+u_{x}=0 \\
u_{y}+u_{x}-u_{x x}=0 \\
u_{y}+u_{x}-u_{x x x}=0 \\
u_{y}+u u_{x}=0
\end{array}
$$

para o caso de EDPs de primeira ordem não lineares existe uma equação que tem a forma

$$
u_{y}+c(u) u_{x}=0
$$

denominada equação cinemática ou também equação da onda cinemática, esta equação descreve a propagação de ondas não lineares. A solução dos problemas não lineares traz complicações pois nem sempre é garantida a unicidade da solução. Consideraremos a solução analítica e numérica de alguns casos particulares que permitam verificar este fato.

Exemplo 4.20 A equação de onda linear

$$
u_{y}+u_{x}=0
$$

é um caso particular da equação de transporte que foi revisada no Capitulo 1, para o caso particular em que $a=1$, temos que o problema de valor inicial

$$
\begin{gathered}
u_{y}+u_{x}=0 \\
u(x, 0)=f(x)
\end{gathered}
$$

tem solução $u(x, y)=f(x-y)$.

Para $f(x)=1-x$, temos que a solução é o plano $u(x, y)=1-x+y$, e as curvas características são mostradas nas Figuras 4.36(a), 4.36(b).

Se tomamos $f(x)=\sin (\pi x)$, a solução da equação é dada por $u(x, y)=\sin (\pi(x-y))$ e sua solução numérica é mostrada nas Figuras 4.37(a), 4.37(b), 4.38(a), 4.38(b),

Exemplo 4.21 A equação

$$
\begin{aligned}
& u_{y}+u^{2} u_{x}=0 \\
& u(x, 0)=1-x
\end{aligned}
$$

tem como solução analítica 


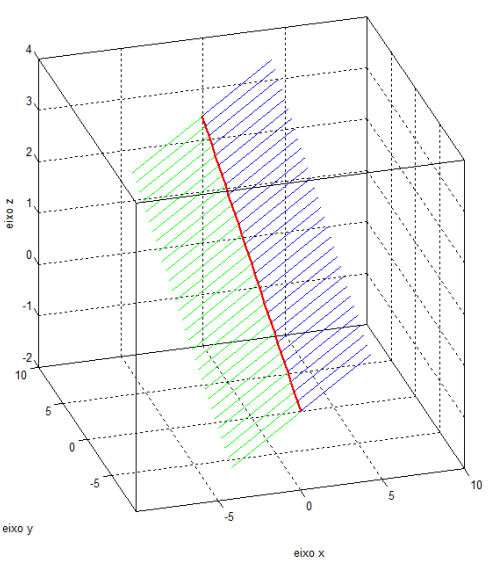

(a) Curvas Características

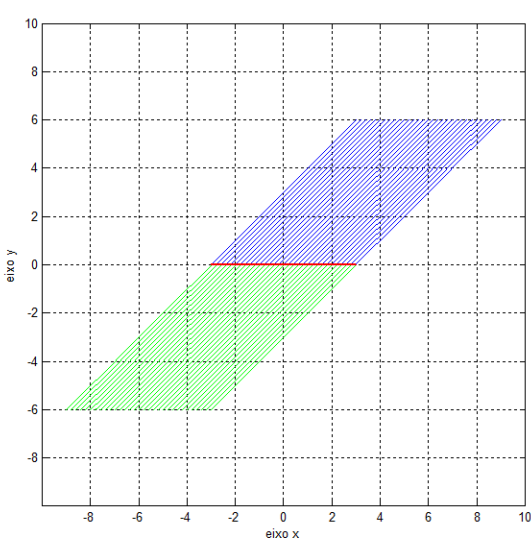

(b) Curvas Características No plano $R^{2}$

Figura 4.36: Solução numérica da equação $u_{x}+u_{y}=0$, com $u(x, 0)=1-x$, usando o método de Runge-Kutta de ordem 5

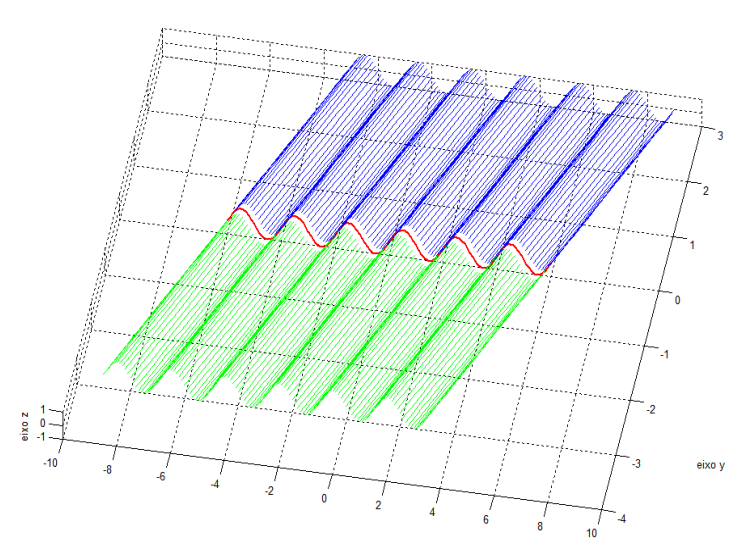

(a) Curvas Características

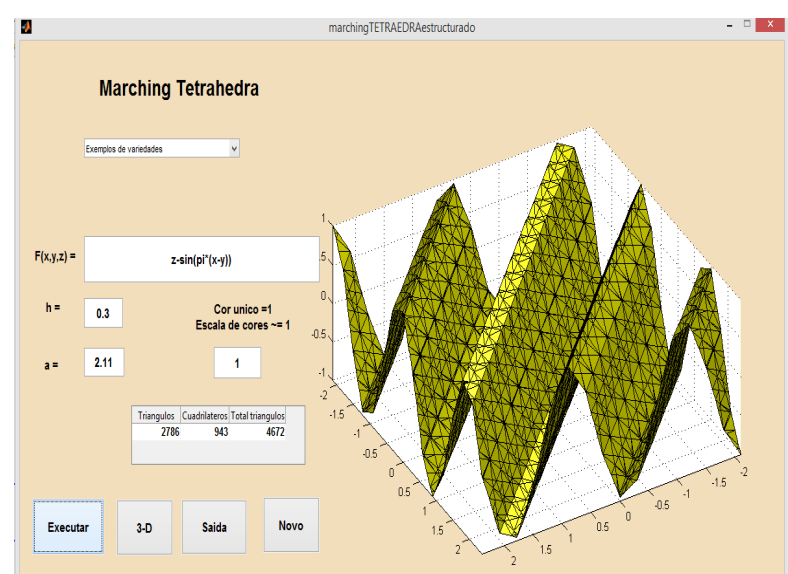

(b) Grafica da Solução analítica

Figura 4.37: Curvas características e solução analítica da equação $u_{x}+u_{y}=0$, com

$$
\begin{gathered}
u(x, 0)=\sin (\pi x) \\
u(x, y)=\frac{1-\sqrt{1-4 y(1-x)}}{2 y} \\
\left\{(x, y) \in \mathbf{R}^{2}:(1-x) y<\frac{1}{4} ; y>0\right\}
\end{gathered}
$$

as curvas características são mostradas nas Figuras 4.39, 4.40(a), 4.40(b).

A solução analítica pode-se determinar aproveitando o fato que a equação é quase-linear assim

$$
\begin{cases}\frac{d X}{d s}=U^{2}, & X(0)=t \\ \frac{d Y}{d s}=1, & Y(0)=0 \\ \frac{d U}{d s}=0, & U(0)=1-t\end{cases}
$$




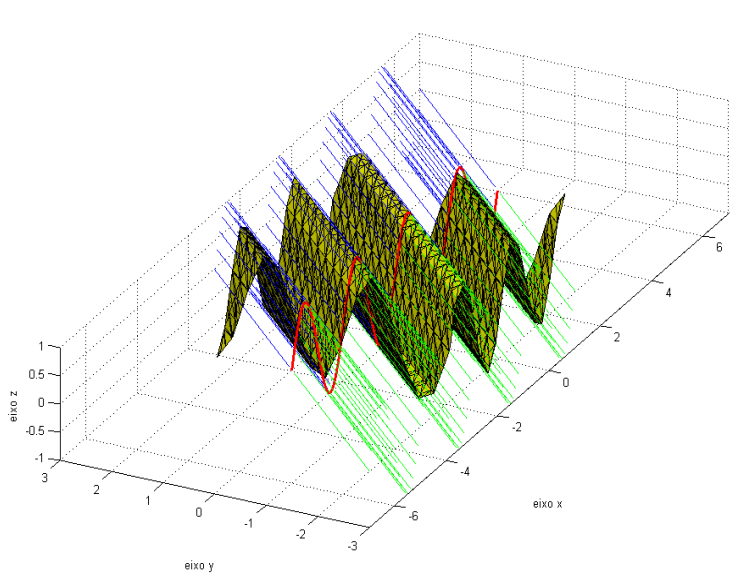

(a) Curvas Características

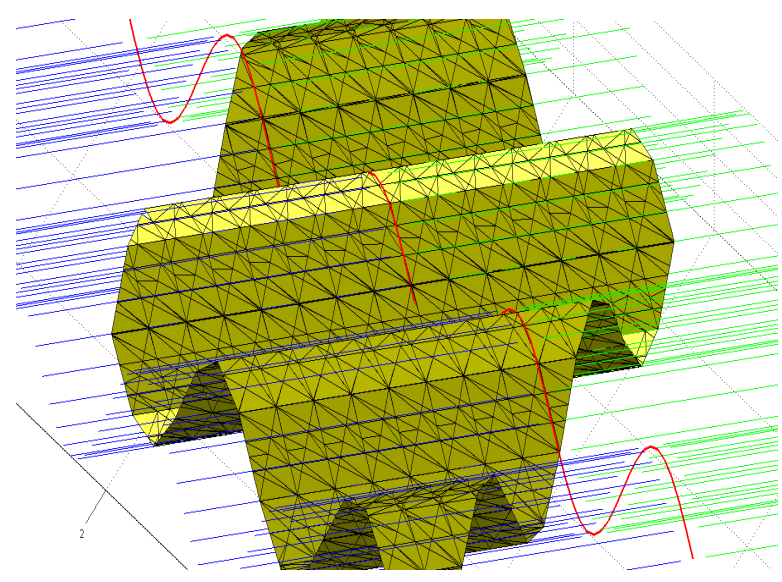

(b) Curvas Características

Figura 4.38: Comparação da Solução analítica e as curvas características da equação

$$
u_{x}+u_{y}=0, \operatorname{com} u(x, 0)=\sin (\pi x)
$$

- $\frac{d X}{d s}=U^{2}$, sendo $\frac{d U}{d s}=0$ então

$$
\begin{aligned}
U & =C \\
U(s, t) & =1-t
\end{aligned}
$$

$\log 0$

$$
\begin{aligned}
\frac{d X}{d s} & =(1-t)^{2} \\
X & =(1-t)^{2} s+C_{1} \\
X(s, t) & =(1-t)^{2} s+t
\end{aligned}
$$

- $\frac{d T}{d s}=1 \Rightarrow Y(s, t)=s$

- $U(s, t)=1-t$

$$
\begin{aligned}
X & =(1-t)^{2} Y+t \\
\frac{X}{Y} & =(t-1)^{2}+\frac{t-1}{Y}+\frac{1}{Y} \\
\frac{X}{Y} & =\left(t-1+\frac{1}{2 Y}\right)^{2}-\frac{1}{4 Y^{2}}+\frac{1}{Y} \\
\frac{4 Y(X-1)+1}{4 Y^{2}} & =\left(t-1+\frac{1}{2 Y}\right)^{2} \\
t & =1-\frac{1}{2 Y} \pm \frac{\sqrt{4 Y(X-1)+1}}{2 Y}, \text { se } Y>0
\end{aligned}
$$


finalmente como $U(s, t)=1-t$ temos

$$
u(x, y)=\frac{1}{2 y}\{1 \pm \sqrt{1-4 y(1-x)}\}
$$

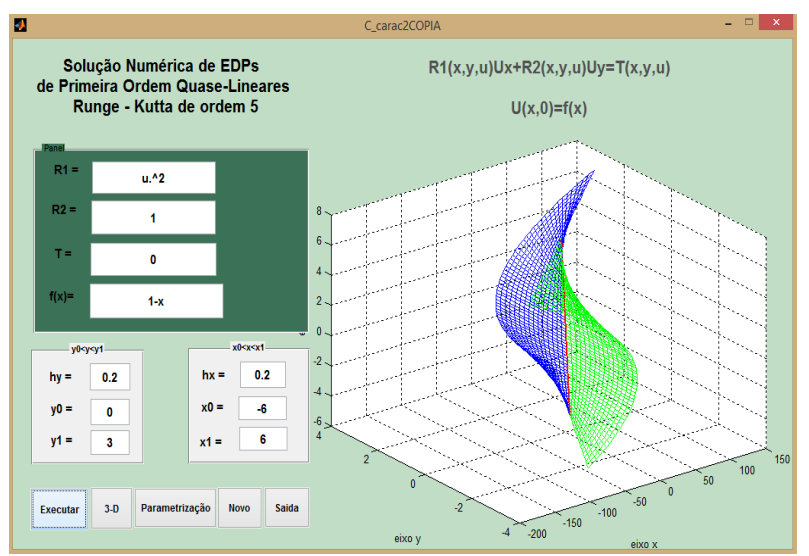

Figura 4.39: Solução numérica da equação $u_{y}+u^{2} u_{x}=0, \operatorname{com} u(x, 0)=1-x$, usando o Método de Runge-Kutta de ordem 5

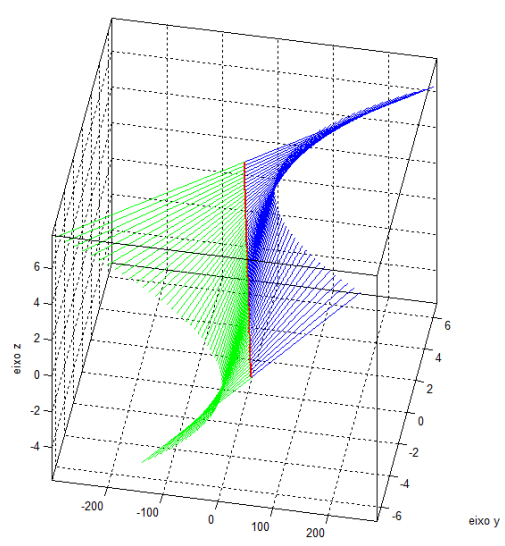

eixox

(a) Curvas Características

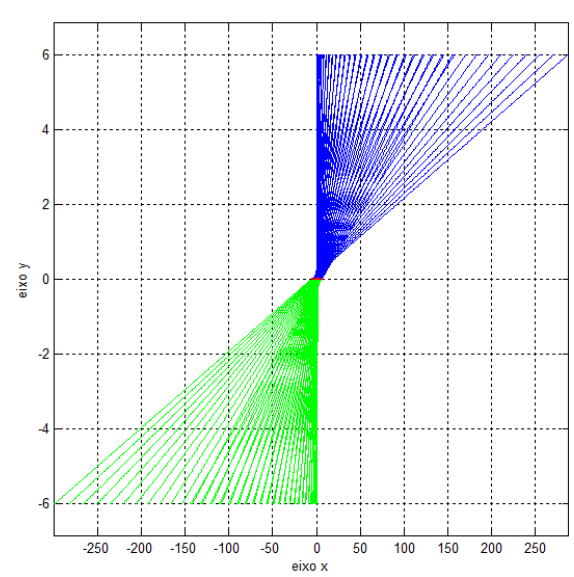

(b) Curvas características no plano $R^{2}$

Figura 4.40: Solução numérica da equação $u_{y}+u^{2} u_{x}=0$, com $u(x, 0)=1-x$, usando o Método de Runge-Kutta de ordem 5 
Equação de Burgers não viscosa Um caso particular da equação cinemática é dado quando $c(u)=u$, definindo a equação

$$
u_{y}+u u_{x}=0
$$

denominada equação de Burgers não viscosa, esta equação é um modelo matemático que descreve fenômenos tais como o transporte de partículas, o rompimento da barrera do sonido por um avião supersônico, entre outros, considerando como dado inicial $u(x, 0)=f(x)$ este modelo tem como solução $u=f(x-y u)$, se interpretamos a equação 4.5 como o transporte de partículas, esta equação da garantia de que qualquer partícula do fluido tem aceleração nula, assim a velocidade é constante ao longo das trajetórias do fluido.

Ao longo de cada curva característica $u$ é constante. Devido a isto as características são linhas retas determinadas desde a curva inicial.

Este tipo de modelo não linear mostra que dependendo da condição inicial, a equação pode não ter uma solução clássica que satisfaça o teorema de Cauchy, esta equação mostra que a existência e unicidade é de carácter local.

Como a solução é da forma $u=f(x-y u)$ e se a sua vez representa uma onda, então a forma da onda depende também de $u$, sendo $u(x, 0)=f(x)$ a forma inicial da onda. O valor numérico de $u(x, y)$ determina a altura da onda, e o movimento da onda esta dado pelo sistema característico

$$
\left\{\begin{array}{c}
\frac{d X}{d s}=u \\
\frac{d Y}{d s}=1 \\
\frac{d U}{d s}=0
\end{array}\right.
$$

notamos que a velocidade de propagação não é constante pois $\frac{d X}{d s}=u$, além disso, a propagação não é unidirecional pois partes da onda podem ir para a esquerda, direita ou permanecer igual dependendo se $u<0, u>0$, ou $u=0$, por exemplo se $u>0$ para os valores de $x$ onde $u$ é grande e a onda é alta moverão-se mais rápido que os valores de $x$ onde $u$ tem um valor menor e a onda é mais baixa, portanto pode produzir-se a quebra da onda, este fenômeno é conhecido como ondas de "choque" (Rhee e Amundson, 1986).

Exemplo 4.22 A equação

$$
u_{y}+u u_{x}=0
$$

foi resolvida no Capitulo 1, Exemplo 1.12, determinamos que sua solução é $u=f(x-y u)$, considerando o problema de valor inicial

$$
\begin{gathered}
u_{y}+u u_{x}=0 \\
u(x, 0)=1-x
\end{gathered}
$$

temos que sua solução é $u=1-x+y u$, e as curvas características são mostradas nas Figuras 4.41(a), 4.41(b), 4.42(a), 4.42(b), 4.43. 


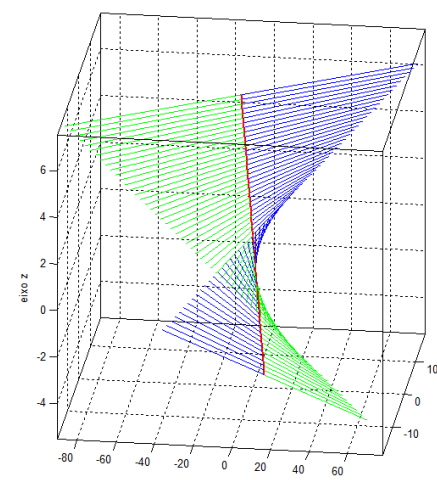

(a) Curvas Características

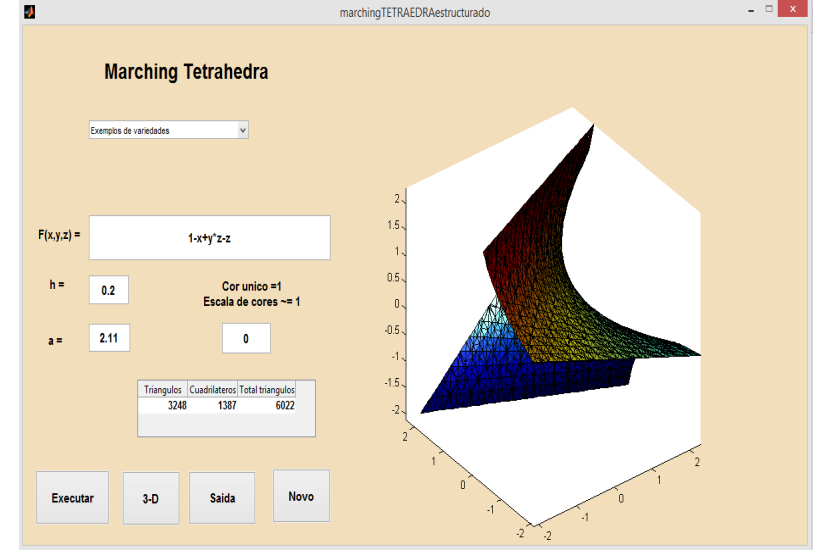

(b) Solução analítica

Figura 4.41: Solução numérica da equação $u_{y}+u u_{x}=0$, com $u(x, 0)=1-x$, usando Runge-Kutta de ordem 5

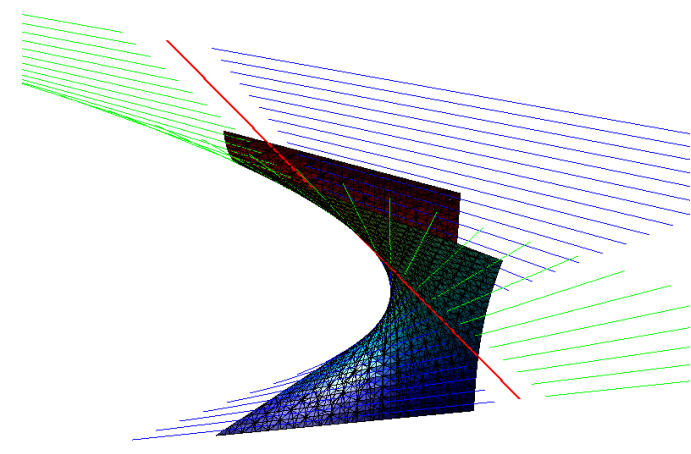

(a) Comparação da solução anailitica e numérica

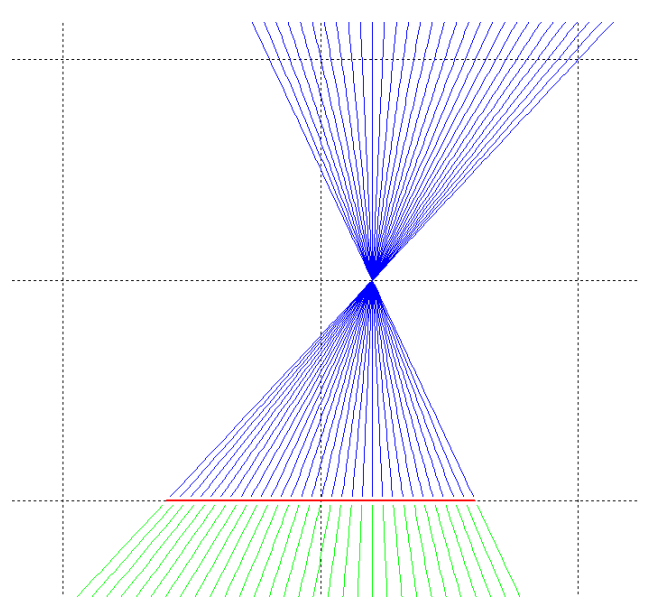

(b) Curvas características no plano $R^{2}$

Figura 4.42: Solução numérica da equação $u_{y}+u u_{x}=0$, com $u(x, 0)=1-x$, usando Runge-Kutta de ordem 5

Exemplo 4.23 Dada a equação

$$
\begin{gathered}
u_{y}+u u_{x}=0 \\
u(x, 0)=\sin (\pi x)
\end{gathered}
$$

que tem solução $u=\sin (\pi(x-y u))$, mostramos as curvas características e a solução analítica nas Figuras 4.46(a), 4.46(b).

Nos exemplos expostos notamos que a forma da onda depende do dado inicial, assim como a produção de uma quebra da onda. Assim é possível achar o tempo de quebra da onda que denotaremos por $y_{0}$, com este objetivo deve-se determinar a pendente da forma de onda $u=$ 


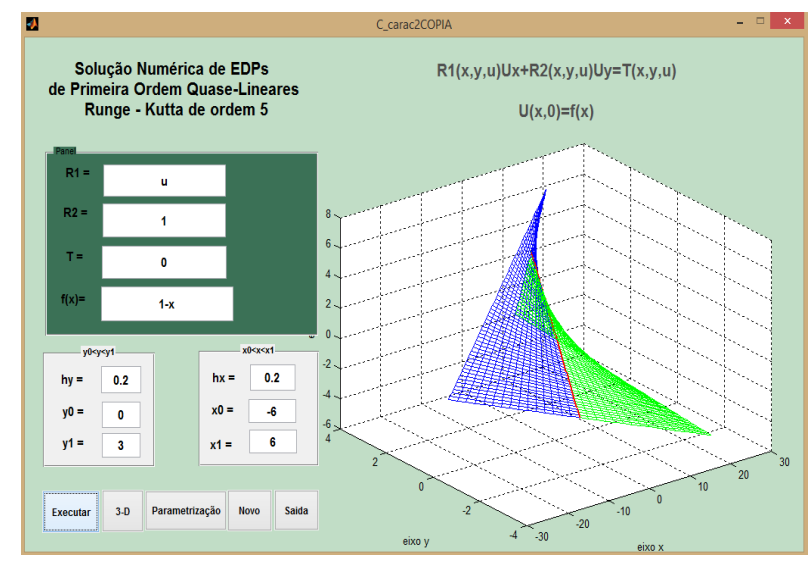

Figura 4.43: Parametrização da solução numérica para a equação $u_{y}+u u_{x}=0$, com $u(x, 0)=1-x$, usando o Método de Runge-Kutta de ordem 5

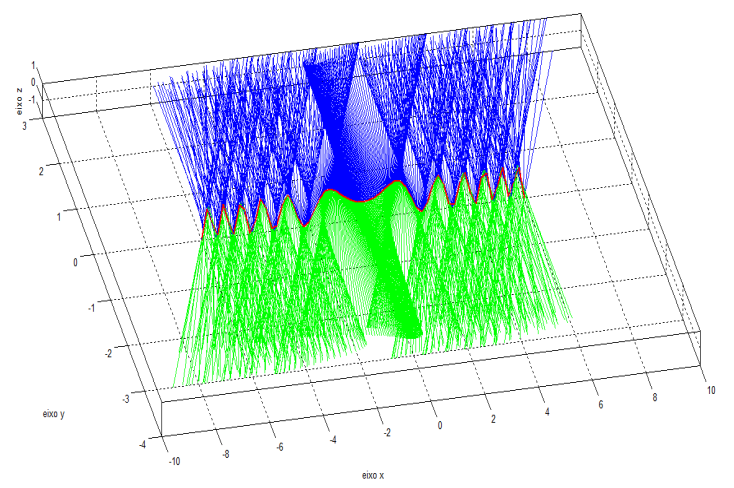

(a) Curvas Características No plano $R^{2}$

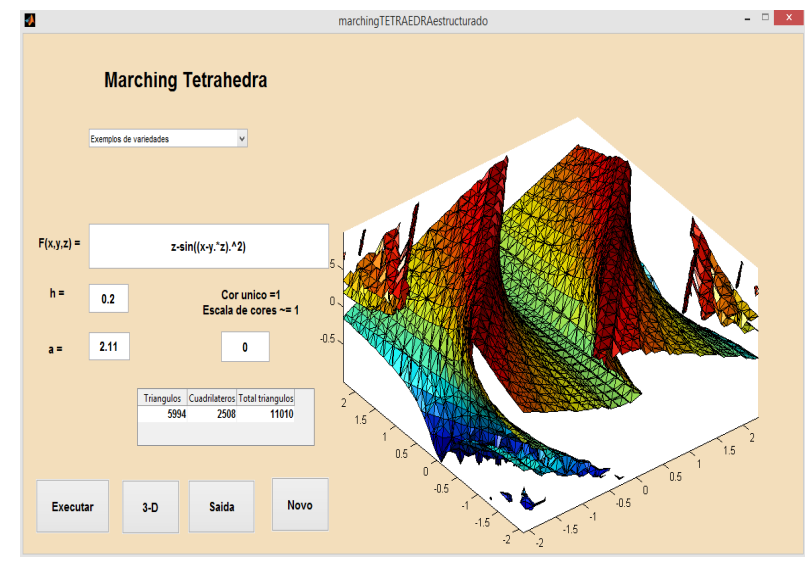

(b) Solução analítica

Figura 4.44: Solução analítica e Solução numérica da equação $u_{y}+u u_{x}=0$, sendo $u(x, 0)=\sin \left(x^{2}\right)$, usando o Método de Runge-Kutta de ordem 5

$f(x-y u)$ no tempo $y$, derivando implicitamente obtêm-se

$$
\frac{\partial u}{\partial x}=\frac{f^{\prime}(X-Y U)}{1+Y f^{\prime}(X-Y U)}
$$

como $X(s, t)=t+s f(t)$ e $Y(s, t)=s$ temos que as curvas características projetadas no plano $R^{2}$ são dadas pelas retas $X-Y f(t)=t$, sendo assim, quando $1+Y f^{\prime}(X-Y U)=1+Y f^{\prime}(t)$ anula-se então $\frac{\partial u}{\partial x} \rightarrow \infty$ porém a quebra de onda é dado depois de $y_{0}=\frac{1}{f^{\prime}(t)}$.

Exemplo 4.24 Dada a equação

$$
\begin{aligned}
& u_{y}+u u_{x}=0 \\
& u(x, 0)=e^{-x^{2}}
\end{aligned}
$$




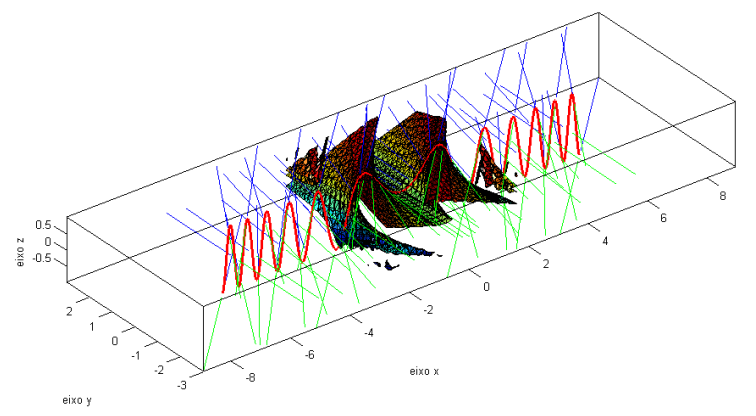

(a) Curvas Características

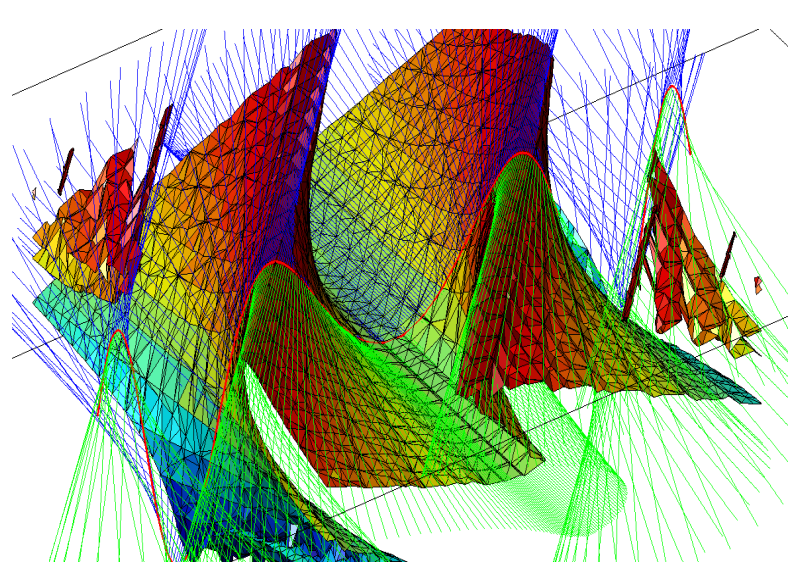

(b) Curvas características

Figura 4.45: Comparação da Solução analítica com as curvas características da equação

$$
u_{y}+u u_{x}=0 \text {, sendo } u(x, 0)=\sin \left(x^{2}\right)
$$

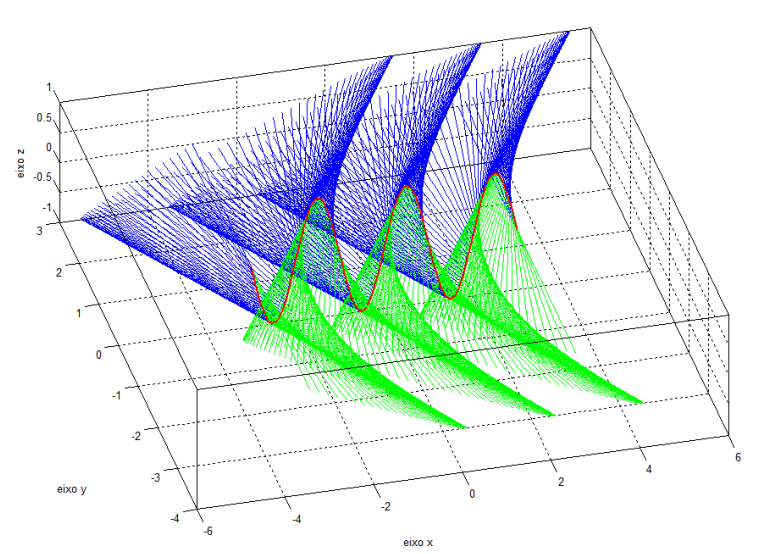

(a) Curvas Características

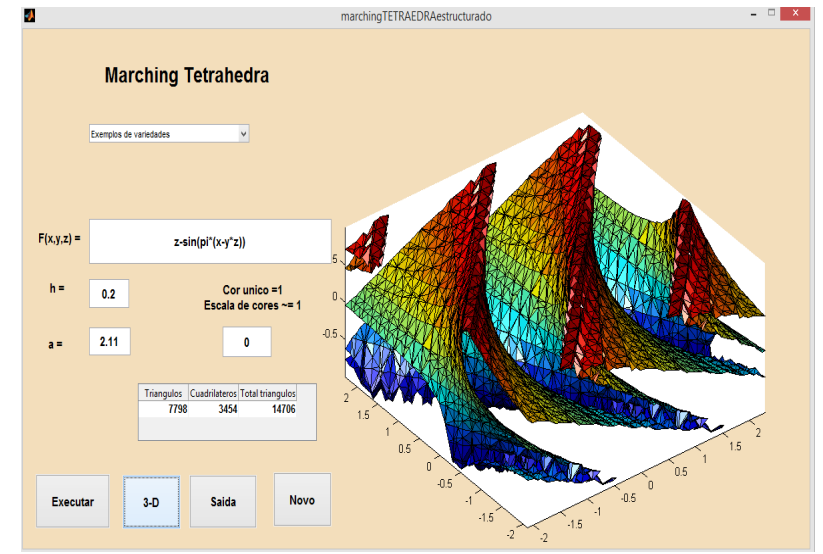

(b) Solução analítica

Figura 4.46: Solução numérica da equação $u_{y}+u u_{x}=0, \operatorname{com} u(x, 0)=\sin (\pi x)$

que tem solução $u=e^{-(x-y u)^{2}}$, mostramos o efeito da onda viajante considerando a curva inicial $f(x)=e^{-x^{2}}$,assim como as curvas características e os choques produzidos nas Figuras 4.51(a), 4.51(b), 4.52(a), 4.52(b).

Exemplo 4.25 Para o problema de valor inicial dado por

$$
\begin{aligned}
& u_{y}+u u_{x}=0 \\
& u(x, 0)=e^{-x^{2}}
\end{aligned}
$$

as curvas características projetadas no plano se cruzam. É possível determinar o tempo de choque como indicam as Figuras 4.53(a), 4.53(b) assim sendo $c(u)=u$ e considerando $c(u)>0, c^{\prime}(u)>$ 0 e $f^{\prime}(x) \in C^{1}$ definindo $h(t)=c(f(t))$ temos que: 


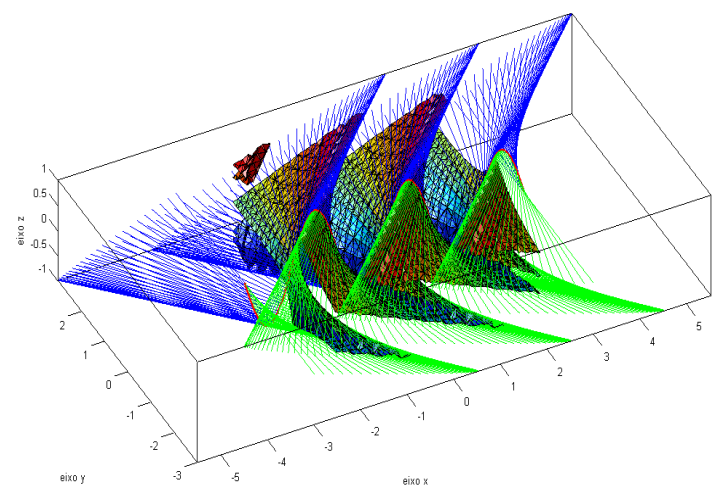

(a) Curvas características

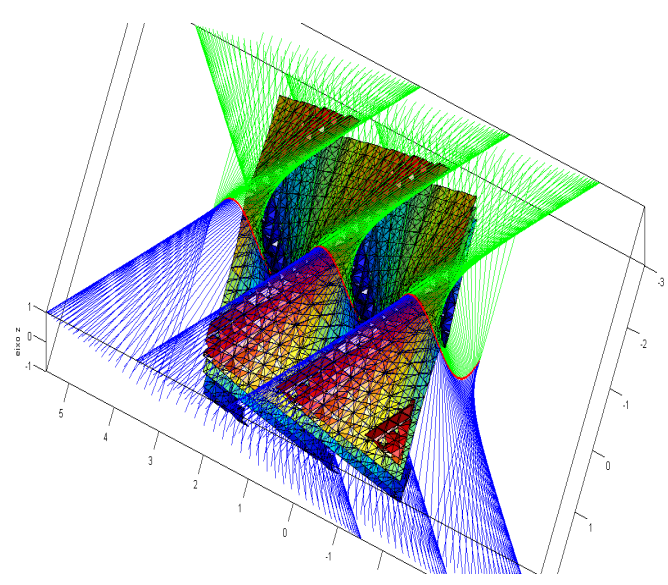

(b) Curvas caracteristicas vista superior

Figura 4.47: Solução numérica da equação $u_{y}+u u_{x}=0, \operatorname{com} u(x, 0)=\sin (\pi x)$

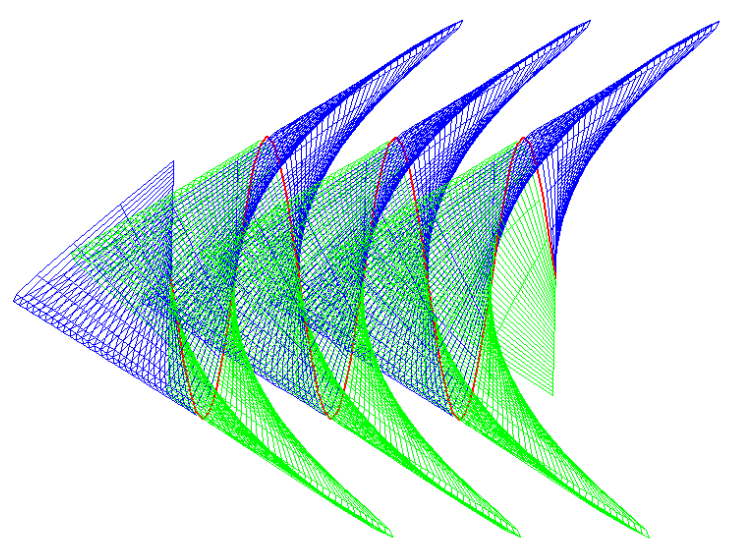

Figura 4.48: Parametrização da solução

$h(t)=e^{-t^{2}} \Rightarrow h^{\prime \prime}(t)=0$ quando $t_{0}=\sqrt{1 / 2} \approx 0.7071, \log 0 y_{0}=-\frac{1}{f^{\prime}\left(t_{0}\right)}=1.16$, podemos ver o ponto de quebra nas Figuras 4.54(a), 4.54(b).

Uma solução de uma EDP não pode tomar valores múltiplos pois não seria função, em consequência, a solução achada não é clássica, isto é a equação de Burgers não poderá ter uma solução de classe $C^{1}$ depois do tempo quando a onda se quebra. 


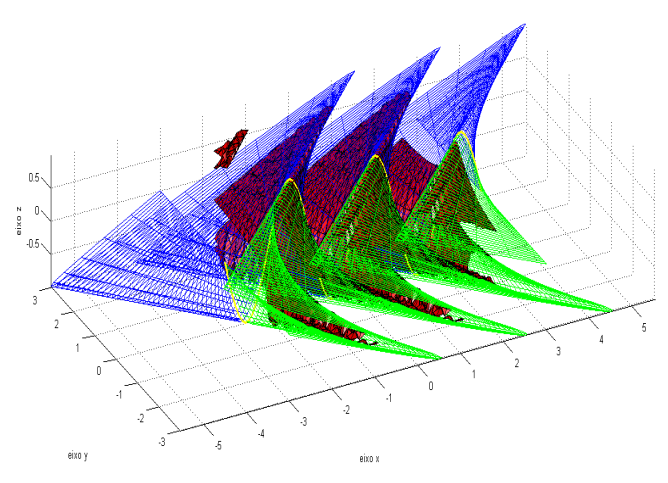

(a) Parametrização da solução

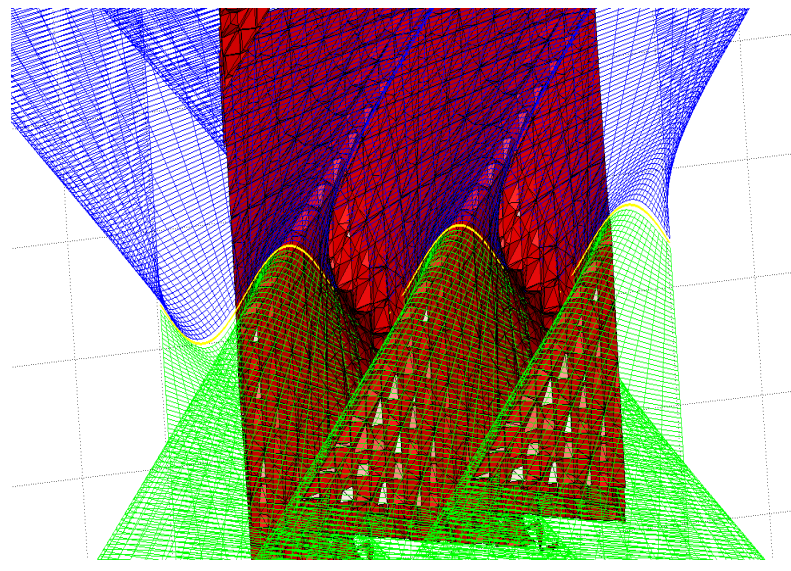

(b) Parametrização da solução vista superior

Figura 4.49: Comparação das curvas características e a solução analítica da equação $u_{y}+u u_{x}=0, \operatorname{com} u(x, 0)=\sin (\pi x)$

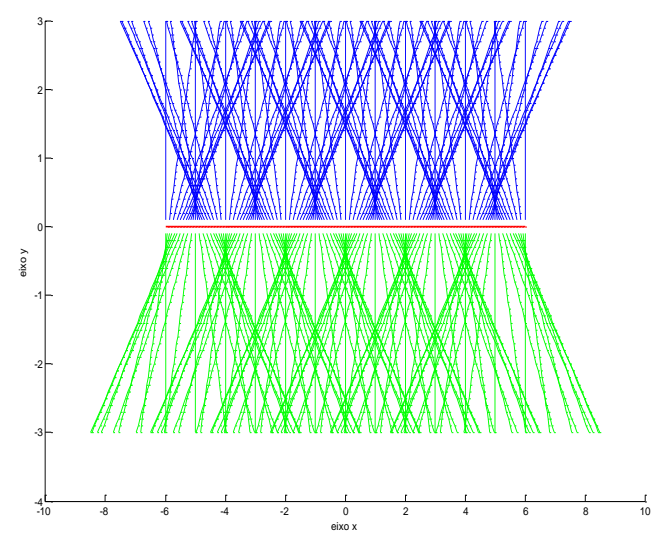

Figura 4.50: Vista superior das curvas características

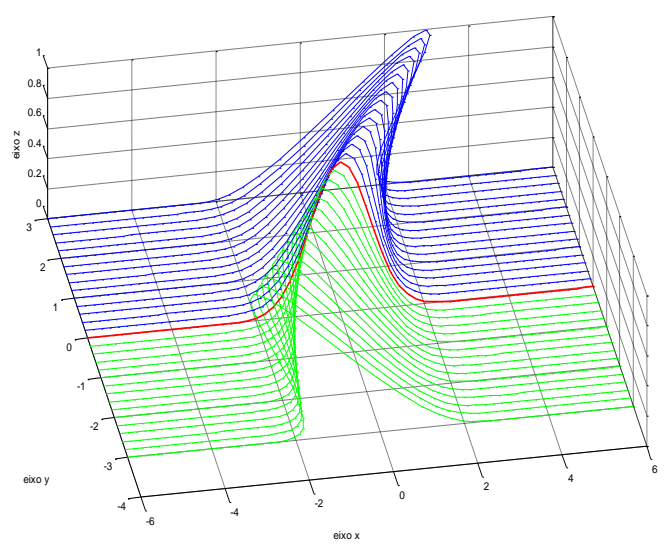

(a) Onda viajante

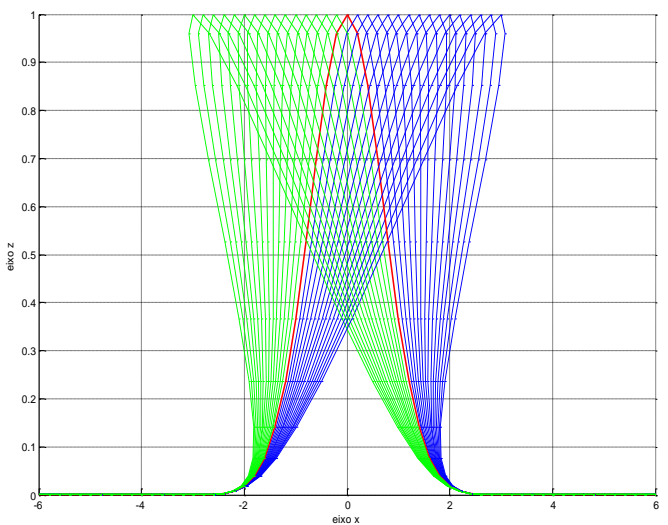

(b) Onda viajante vista frontal plano $x u$

Figura 4.51: Onda viajante da equação $u_{y}+u u_{x}=0, \operatorname{com} u(x, 0)=e^{-x^{2}}$ 


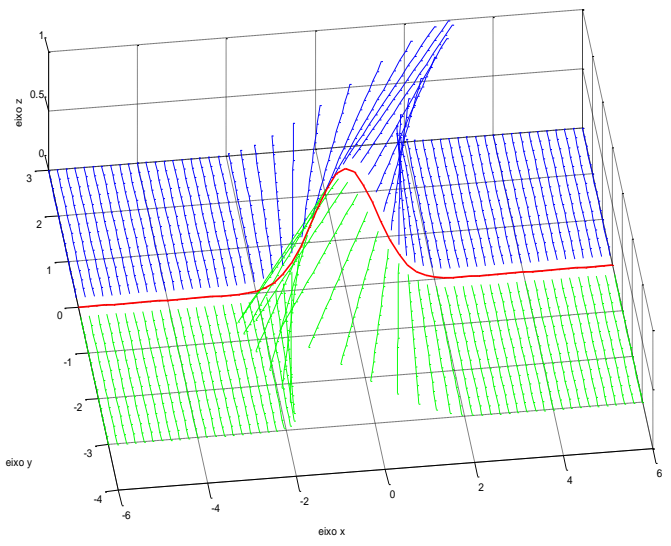

(a) Curvas Características

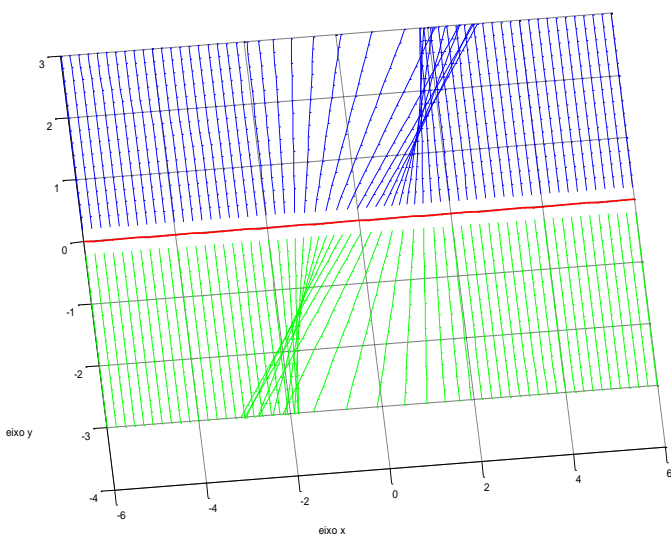

(b) Projeção de curvas características no plano $R^{2}$ analítica

Figura 4.52: Curvas características e choques da equação $u_{y}+u u_{x}=0, \operatorname{com} u(x, 0)=e^{-x^{2}}$

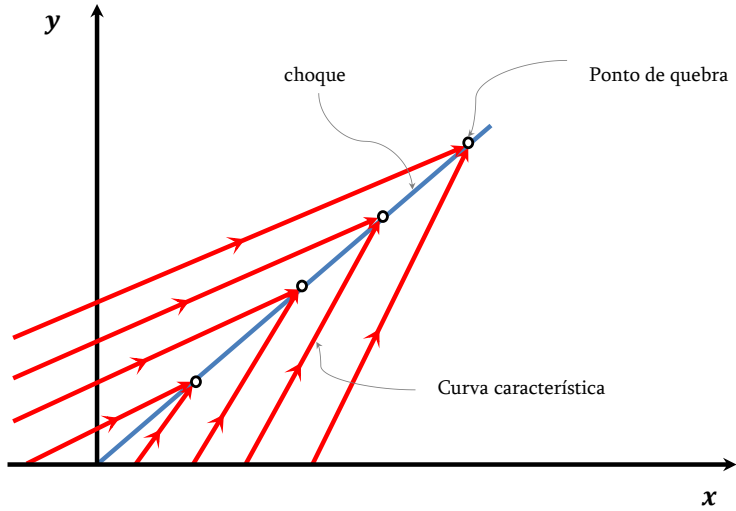

(a) Ruta de choque $R^{2}$

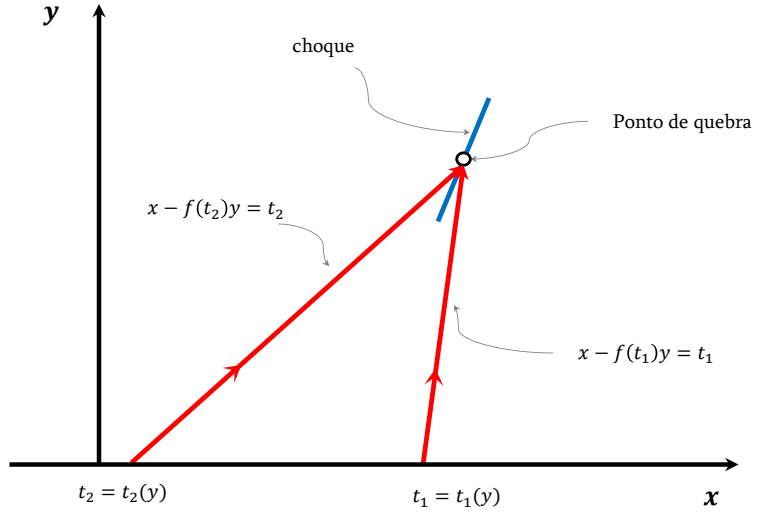

(b) Ponto de quebra características

Figura 4.53: Formação de choques

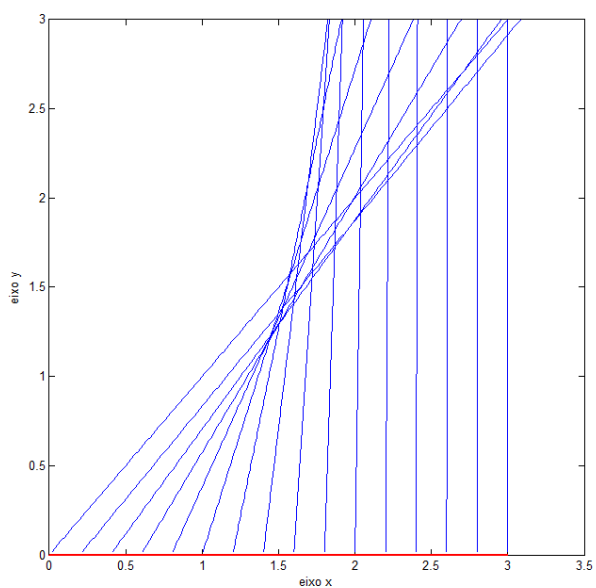

(a) Curvas caracteristicas projetadas $R^{2}$

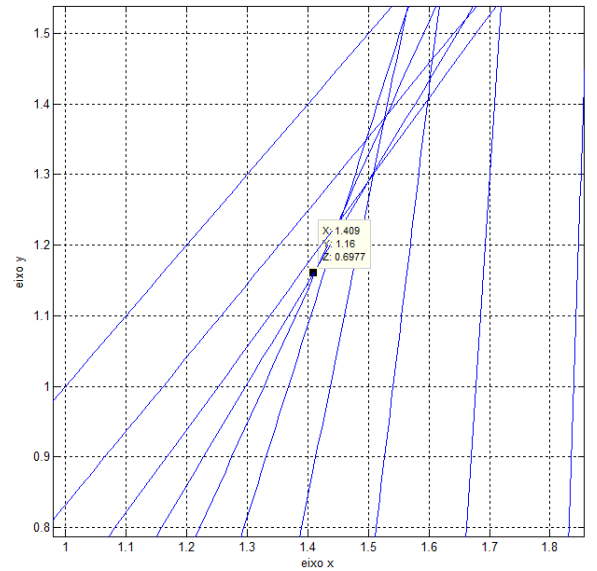

(b) Ponto de quebra características

Figura 4.54: Formação de choques $u_{y}+u u_{x}=0$, sendo $u(x, 0)=e^{-x^{2}}$ 


\subsection{Equação de Hamilton Jacobi}

Dada uma EDP implícita de primeira ordem

$$
F\left(x_{1}, \ldots, x_{n-1}, x_{n}, u, u_{x_{1}}, \ldots, u_{x_{n-1}}, u_{x_{n}}\right)=0
$$

considerando o caso particular em que $F$ não dependa explicitamente de $u$ e possa-se descrever na forma

$$
F=u_{x_{n}}+H\left(x_{1}, \ldots, x_{n-1}, x_{n}, u_{x_{1}}, \ldots, u_{x_{n-1}}\right)
$$

denotando por $t=x_{n}$, e $H$ é o Hamiltoniano obtemos a equação denominada Equação de Hamilton-Jacobi

$$
u_{t}+H\left(x_{1}, \ldots, x_{n-1}, t, u_{x_{1}}, \ldots, u_{x_{n-1}}\right)=0
$$

considerando a notação $x=\left(x_{1}, \ldots, x_{n-1}\right)$, e $\nabla_{x} u=\left(u_{x_{1}}, \ldots, u_{x_{n-1}}\right)$ formulamos o seguinte problema de valor inicial

$$
\left\{\begin{array}{l}
u_{t}+H\left(x, t, \nabla_{x} u\right)=0, \\
u(x, 0)=g(x)
\end{array}\right.
$$

sendo $g: \mathbf{R}^{n-1} \rightarrow \mathbf{R}$ é uma função contínua, uma solução clássica de 4.8 é uma função $u: \mathbf{R}^{n} \rightarrow \mathbf{R}$ de classe $C^{1}$ que satisfaz esta equação.

Ao denotar $p_{i}=u_{x_{i}}$, o sistema característico para o problema de valor inicial 4.8 é dado por

$$
\begin{array}{rlr}
\frac{d x_{i}}{d \tau} & =F_{p_{i}}=\frac{\partial H}{\partial p_{i}} & \\
\frac{d t}{d \tau} & =F_{p_{n}}=F_{u_{t}}=1 & \\
\frac{d u}{d \tau} & =\sum_{i=1}^{n-1} p_{i} F_{p_{i}}+p_{n} F_{p_{n}}=\sum_{i=1}^{n-1} p_{i} \frac{\partial H}{\partial p_{i}}+p_{n} & \\
\frac{d p_{i}}{d \tau} & =-\left(F_{x_{i}}+p_{i} F_{u}\right)=-\frac{\partial H}{\partial x_{i}} & \\
\frac{d p_{n}}{d \tau} & =-\left(F_{t}+p_{n} F_{u}\right)=-\frac{\partial H}{\partial t} &
\end{array}
$$

como $\frac{d t}{d \tau}=1$ usando a condição inicial $t(\tau)=0$ em $\tau=0$ temos que $t=\tau$. Por tanto obtemos o sistema

$$
\left\{\begin{aligned}
\frac{d x_{i}}{d t} & =\frac{\partial H}{\partial p_{i}} \\
\frac{d p_{i}}{d t} & =-\frac{\partial H}{\partial x_{i}}
\end{aligned}\right.
$$

que permite determinar $x, \nabla_{x} u$ e o sistema 4.9 é chamado sistema canônico. 
Por outro lado o sistema 4.10

$$
\left\{\begin{array}{l}
\frac{d p_{n}}{d t}=-\frac{\partial H}{\partial t} \\
\frac{d u}{d t}=\sum_{i=1}^{n-1} p_{i} \frac{\partial H}{\partial p_{i}}-H
\end{array}\right.
$$

permite achar $p_{n}$ e $u$, utilizando para isso a solução do sistema 4.9.

Exemplo 4.26 O Hamiltoniano para um oscilador harmônico unidimensional é dado pela expressão

$$
H=\frac{p^{2}}{2 m}+\frac{k}{2} q^{2}
$$

onde $m$ é a masa da partícula e $k$ é a constante elástica da mola, usando este Hamiltoniano a equação de Hamilton-Jacobi é dada por

$$
S_{t}+\frac{1}{2 m}\left(S_{q}\right)^{2}+\frac{1}{2} k q^{2}=0
$$

para este sistema conservativo o tempo t pode ser separado se o hamiltoniano não depende explicitamente do tempo, assim podemos descrever $S$ como $S(q, t)=u(q)+E t \operatorname{logo}$

$$
\frac{1}{2 m}\left(u_{q}\right)^{2}+\frac{1}{2} k q^{2}=E
$$

daqui

$$
u=\sqrt{m k} \int \sqrt{\left(\frac{2 E}{k}\right)-q^{2}} d q=S+E t
$$

sendo $S$ independente de $E$ temos

$$
\frac{\partial u}{\partial E}=-\sqrt{\frac{m}{k}} \cos ^{-1}\left(q \sqrt{\frac{k}{2 m}}\right)+\tau=t
$$

isolando $q$ temos

$$
\begin{gathered}
q(t)=\sqrt{\frac{2 E}{k}} \cos (\omega(t-\tau)) \quad \text { com } \quad \omega^{2}=\frac{k}{m} \\
p(t)=m \dot{q}=-\sqrt{2 m E} \sin (\omega(t-\tau))
\end{gathered}
$$

é possível chegar as mesmas equações definindo

$$
F\left(q, t, S, S_{q}, S_{t}\right)=S_{t}+\frac{1}{2 m}\left(S_{q}\right)^{2}+\frac{1}{2} k q^{2}=0
$$

utilizando o método de Charpit's e resolvendo as equações

$$
\frac{d q}{S_{q} / m}=d t=\frac{d S}{S_{q}^{2} / m+S_{t}}=\frac{d S_{t}}{0}=\frac{d S_{q}}{-k q}
$$


No entanto as equações de movimento estão definidas pelo sistema canônico

$$
\left\{\begin{array} { l } 
{ \frac { d p } { d t } = \frac { \partial H } { \partial q } } \\
{ \frac { d q } { d t } = - \frac { \partial H } { \partial p } }
\end{array} \Rightarrow \left\{\begin{array}{l}
\frac{d p}{d t}=k q \\
\frac{d q}{d t}=-\frac{p}{m}
\end{array}\right.\right.
$$

podemos usar un método numérico para EDOs e calcular as equações de movimento, por exemplo usando o método de Euler

$$
\left[\begin{array}{l}
p^{k+1} \\
q^{k+1}
\end{array}\right]=\left[\begin{array}{l}
p^{k} \\
q^{k}
\end{array}\right]+h\left[\begin{array}{c}
k q^{k} \\
-\frac{p^{k}}{m}
\end{array}\right]=\left[\begin{array}{cc}
1 & h k \\
-\frac{h}{m} & 1
\end{array}\right]\left[\begin{array}{l}
p^{k} \\
q^{k}
\end{array}\right]
$$

Exemplo 4.27 A Equação de Hamilton-Jacobi para uma partícula livre é determinada pelo Hamiltoniano dado por

$$
H=\frac{1}{2 m}\left(p_{1}^{2}+p_{2}^{2}+p_{3}^{2}\right)+V(x, y, z)
$$

onde $V$ é a energia potencial da partícula, considerando um sistema dinâmico conservativo e escrevendo $S=u-E t$ temos que $u$ satisfaz também a Equação de Hamilton-Jacobi na forma

$$
H\left(q_{i}, \frac{\partial u}{\partial q_{i}}\right)=E
$$

as equações de movimento estão dadas por

$$
p_{i}=\frac{\partial S}{\partial q_{i}}=\frac{\partial S}{\partial q_{i}} \quad i=1,2,3
$$

considerando as coordenadas cartesianas como as coordenas generalizadas temos que

$$
\frac{1}{2 m}\left(\left(\frac{\partial u}{\partial x}\right)^{2}+\left(\frac{\partial u}{\partial y}\right)^{2}+\left(\frac{\partial u}{\partial z}\right)^{2}\right)+V(x, y, z)=E
$$

ou

$$
u_{x}^{2}+u_{y}^{2}+u_{z}^{2}=f(x, y, z)
$$

sendo $f(x, y, z)=2 m(E-V)$, a equação pode ser resolvida pelo método das características usando uma curva inicial de forma similar como vimos no Exemplo 1.14 do Capitulo 1. 


\subsection{Algumas aplicações na Mecânica e Ótica}

A equação da eikonal é uma EDP não linear de primeira ordem, que modela a propagação de ondas de luz ao longo de raios com uma velocidade dada por $c(x, y)$. As superfícies de fase dadas quando $u(x, y)=$ constante são chamadas frentes de onda, as quais são ortogonais aos raios da luz. É possível estabelecer que a equação da eikonal tem a forma

$$
u_{x}^{2}+u_{y}^{2}=n^{2}(x, y)
$$

onde o índice de refração $n$ é definido como $n=\frac{1}{c}$.

A equação pode ser resolvida pelo método das características usando uma curva inicial, como já foi visto. As curvas caraterísticas representam os raios de luz e por sua vez são normais as frentes de onda.

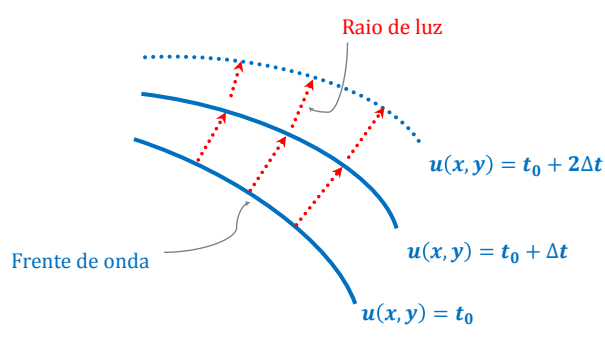

(a) Frentes de onda
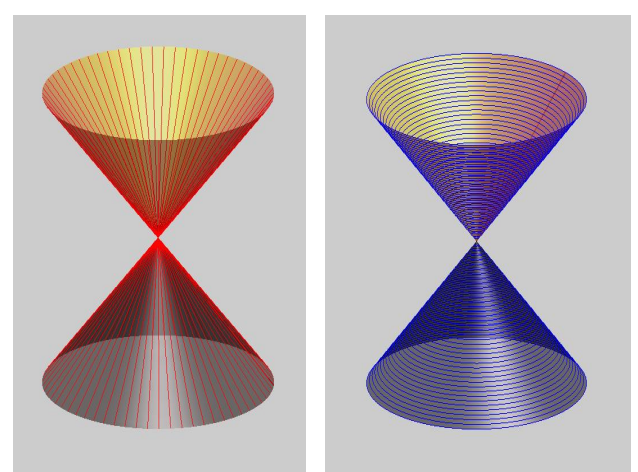

(b) Curvas características e frentes de onda

Figura 4.55: Frentes de onda e curvas características

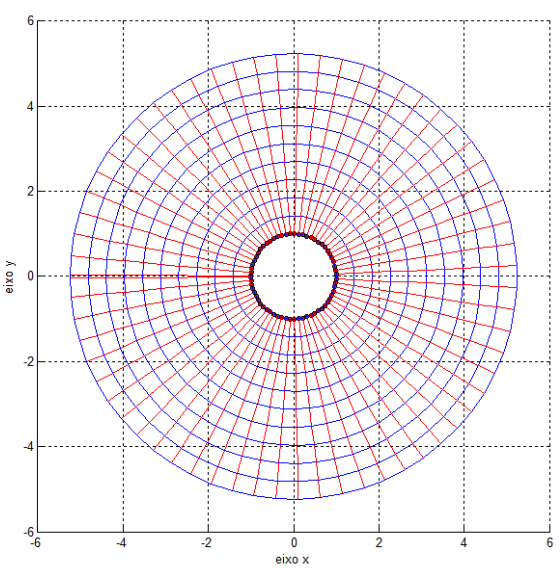

(a) Curvas caraterísticas e frentes de onda em $R^{2}$

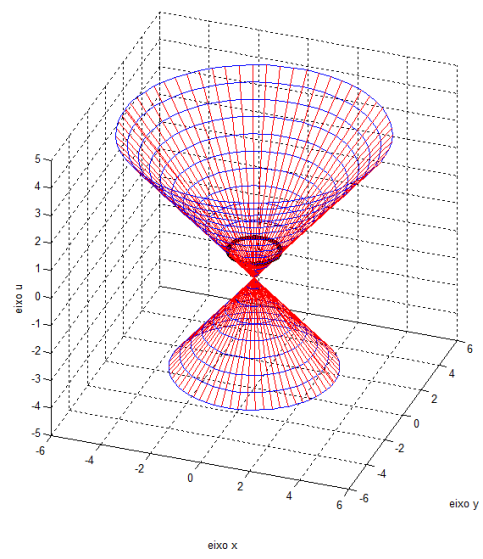

(b) Solução paramétrica

Figura 4.56: Frentes de onda e curvas características correspondentes à equação

$$
\left(u_{x}\right)^{2}+\left(u_{y}\right)^{2}=1, \operatorname{com} x^{2}+y^{2}=1, u=0
$$


Ação: A ação é a magnitude que expressa o produto da energia implicada num processo pelo tempo que dura esse processo.

O principio de Hamilton e a conservação da energia afirmam que a rota de uma partícula deve ser uma geodésica da superfície

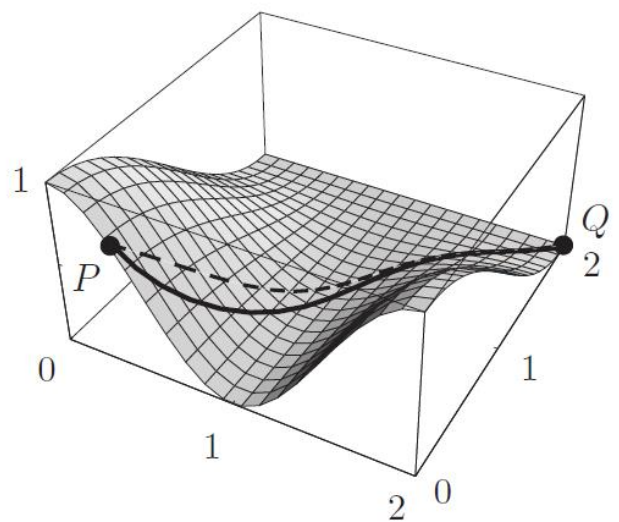

Figura 4.57: Geodésica : Figura retirada de Classical Mechanics, R. Gouglas Gregory

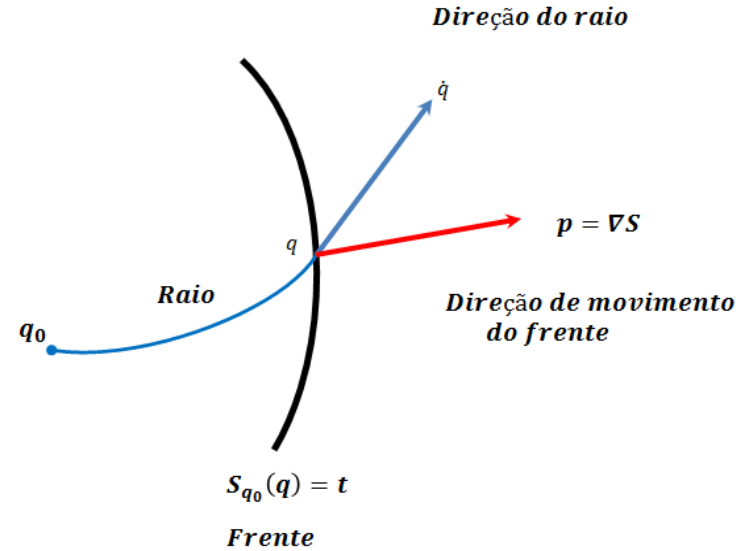

(a) Ação funcional e raios de luz

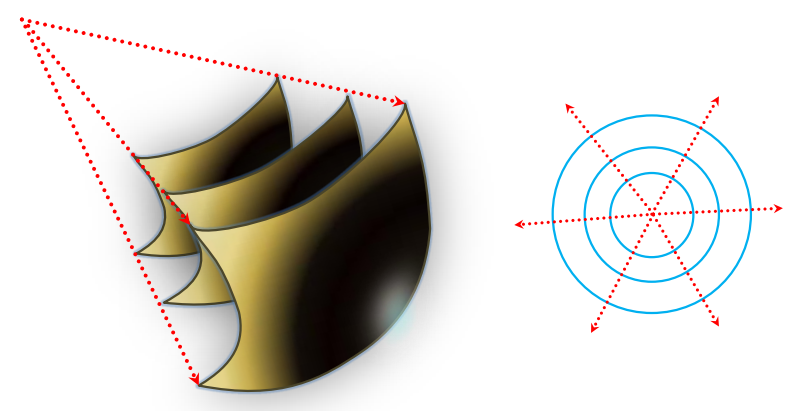

(b) Frentes de onda em $R^{3}$ e $R^{2}$

Figura 4.58: Ação funcional e frentes de onda

Ação funcional A equação de Hamiton-Jacobi estabelece a possibilidade do relacionamento com as aplicações das EDPs implícitas de primeira ordem

A função de ação é definida como

$$
S[q]=\int_{t_{0}}^{t_{1}} L(q, \dot{q}, t) d t,
$$


onde $L(q, \dot{q}, t)$ é o Lagrangiano. Esta função de ação satisfaz a equação de Hamilton-Jacobi

$$
\frac{\partial S}{\partial q}+H\left(q, t, \frac{\partial S}{\partial q}\right)=0
$$

estabelecendo a relação entre as trajetórias de sistemas mecânicos(raios) e as equações diferencias parciais(frentes de onda) como ilustra a Figura 4.58(a). A analogia entre a Mecânica clássica e a ótica, foi estabelecida por Hamilton no ano 1828, o movimento de uma partícula pode ser analisado considerando $S=$ constante propagando-se no espaço como uma frente de onda, as trajetórias da partícula são perpendiculares aos frentes de onda. As trajetórias mecânicas comportam-se igual que os raios da luz na ótica.

Principio de Hamilton O movimento real de um sistema mecânico é equivalente ao extremal de sua ação funcional.

O principio de Hamilton é o principio variacional que é equivalente as equações de movimento de Lagrange.

Existe uma relação entre o Hamiltoniano e o Lagrangiano. Se denotamos por $E$ a energia total, $T$ a energia cinética e por $V$ a energia potencial, sendo $L$ o Lagrangiano e $H$ o Hamiltoniano, as seguintes relações são válidas

$$
L=T-U
$$

e num sistema conservativo,

$$
H=E=T+U
$$

Podendo também estabelecer que o Hamiltoniano e Lagrangiano verificam a equação

$$
H(p, \dot{q})=p \dot{q}-L(q, \dot{q}, t)
$$

Quando uma partícula desliza sobre uma superfície suave $S$, num campo de forças conservativo a soma de sua energia cinética e potencial mantem-se constante no movimento.

Se uma EDP implícita de primeira ordem na forma (4.11) verifica o teorema de Cauchy, então é equivalente ao sistema característico (4.12).

$$
\begin{aligned}
& F\left(x, y, u, u_{x}, u_{y}\right)=0 \\
& \left.u\right|_{\alpha}=\phi
\end{aligned}
$$

Dada uma curva inicial contida na superfície, o sistema característico representa as equações de movimento dos pontos da curva inicial sobre a superfície solução, e as curvas características as trajetórias de cada ponto. 


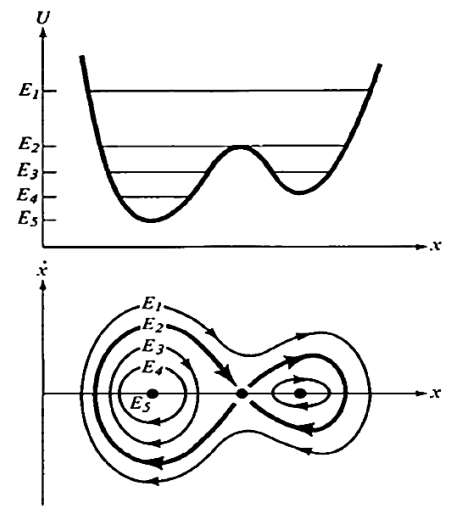

Figura 4.59: Energia Potencial e espaço de fase : Figura retirada de Mathematical Methods of Classical Mechanics, V. I. Arnold

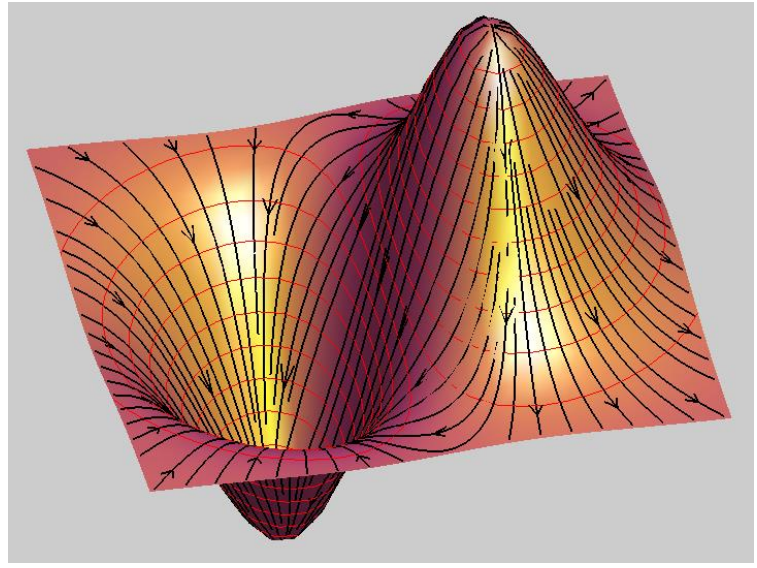

(a) Curvas fase

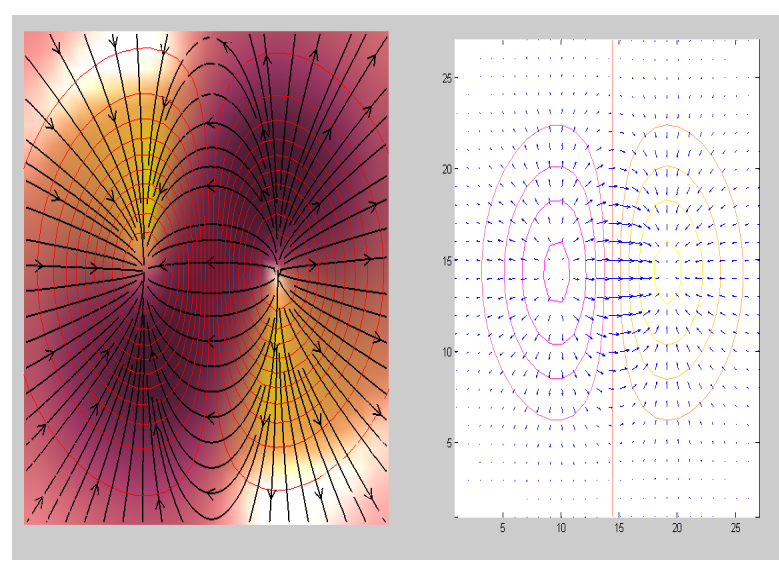

(b) Curvas fase e campo de vetores gradiente

Figura 4.60: Curvas fase e campo de vetores gradiente da função $f(x, y)=x e^{-x^{2}-y^{2}}$

$$
\left\{\begin{array}{lrl}
\frac{d X}{d s} & =F_{p} & X(0)=X(t) \\
\frac{d Y}{d s}=F_{q} & Y(0)=Y(t) \\
\frac{d U}{d s}=p F_{p}+q F_{q} & U(0)=U(t) \\
\frac{d P}{d s}=-F_{x}-p F_{u} & P(0)=P(t) \\
\frac{d Q}{d s}=-F_{y}-q F_{u} & Q(0)=Q(t)
\end{array}\right.
$$

estas equações não necessariamente coincidem com as equações de movimento de Hamilton, pois o sistema pode não ser conservativo. 
Exemplo: Um caso particular da equação de Hamilton Jacobi é dado por

$$
\begin{aligned}
& u_{t}+\frac{1}{2} u_{x}^{2}=0 \\
& u(x, 0)=-x^{2}
\end{aligned}
$$

A equação descreve-se como $F(x, t, u, p, q)=q+\frac{1}{2} p^{2}=0$, usando o sistema característico

$$
\left\{\begin{array}{llrl}
\frac{d X}{d s} & =P & X(0) & =t \\
\frac{d Y}{d s} & =1 & & Y(0)=0 \\
\frac{d U}{d s} & =P^{2}+Q & & U(0)=-t^{2} \\
\frac{d P}{d s} & =0 & & P(0)=-2 t \\
\frac{d Q}{d s} & =0 & & Q(0)=-2 t
\end{array}\right.
$$

Resolvendo o sistema característico usando o Método de Runge-Kutta de ordem 5 obtemos

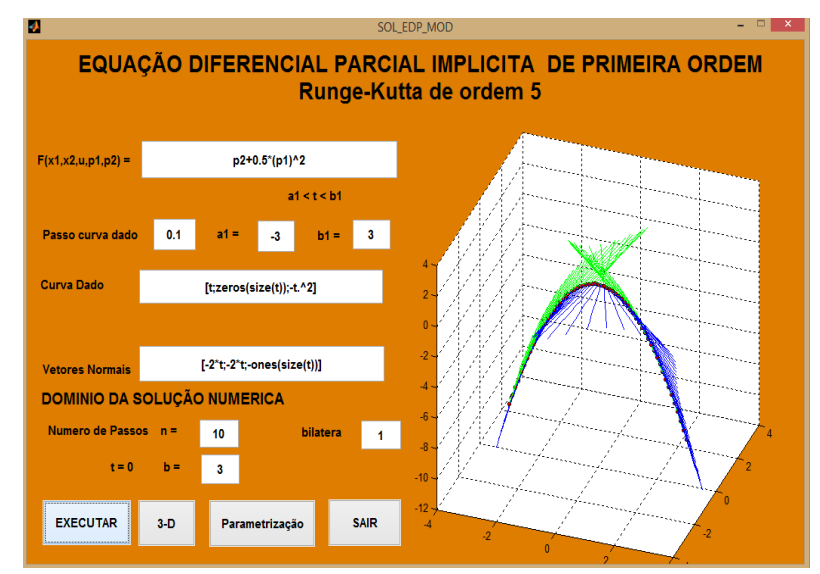

(a) Condições na Interface

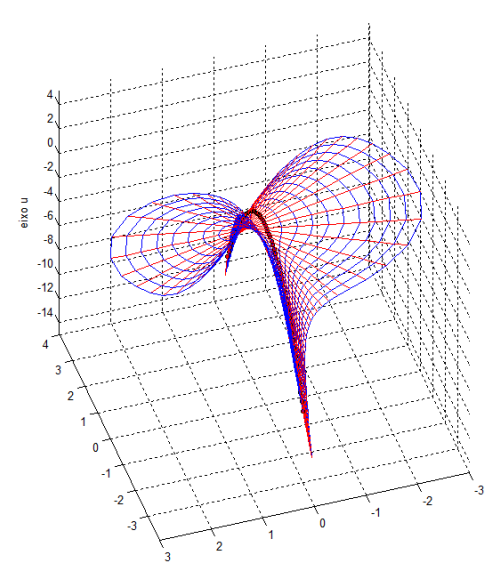

(b) Solução parametrica

Figura 4.61: Solução da equação $u_{t}+\frac{1}{2} u_{x}^{2}=0, \operatorname{com} u(x, 0)=-x^{2}$ 
CAPÍTULO

\section{5}

\section{Conclusões da Dissertação}

O objetivo do método desenvolvido neste trabalho foi obter soluções numéricas de EDPs implícitas de primeira ordem, tendo como condição inicial uma curva dada. Devido à forma ou natureza de uma equação nem sempre as equações têm uma forma apropriada para seu solução. As técnicas utilizadas para resolver EDPs implícitas de primeira ordem empregam diversos métodos para achar a solução de EDOs, estabeleceremos agora as vantagens e limitações do algoritmo desenvolvido.

No desenvolvimento do trabalho foram revisadas diversas bibliografias e técnicas que ofereceram ideias para desenvolver temas relacionados com o projeto, dentro deles o estudo de solução de equações diferenciais sobre variedades propõe aplicações em sistemas mecânicos e sistemas de controle de relevante importância para ser desenvolvidos como trabalhos a futuro. 


\subsection{Conclusões}

1. O método desenvolvido permite resolver uma equação diferencial parcial implícita de primeira ordem, propõe uma alternativa de solução para a solução de EDPs não lineares de primeira ordem.

2. Quando uma EDP de primeira ordem é não linear faz-se necessário dados adicionais para sua solução. Precisa-se dos vetores normais a esta curva.

3. A solução de uma EDP implícita de primeira ordem depende da factibilidade de ter pontos sobre uma curva dada e seus vetores normais nos respectivos pontos.

4. Para a solução numérica de uma EDP de primeira ordem linear é importante conhecer a família de características da EDP antes de ser resolvida analiticamente ou numericamente, pois as condições auxiliares tem que ser bem postas para garantir a existência e unicidade. Para uma solução cujo gráfico esteja contida no espaço $R^{3}$, a visualização da família de características ajuda a determinar se estas características intersectam-se. Isto da informação que diz se a solução será clássica ou se a solução achada toma valores múltiplos (ocorrências de choques).

5. Neste trabalho foi utilizada o Matlab para desenvolver os métodos numéricos apresentados, devido a que oferece um entorno gráfico simples. No entanto devido a que para cada ponto da curva inicial resolvem-se um sistema de ODEs, o custo computacional é alto. O processo pode ficar lento em alguns casos, tendo a alternativa de mudar a outra linguagem como: C, $\mathrm{C}++$, com objetivo de dar maior rapidez à obtenção de uma solução.

6. O teorema generalizado de Cauchy determina em suas hipóteses as condições de factibilidade de estender o método de solução ao caso geral $F\left(x_{1}, x_{2}, \ldots, x_{n}, u, u_{x_{1}}, u_{x_{2}}, \ldots, u_{x_{n}}\right)=0$ onde $F \in C^{2}(\Omega), \Omega \subset \mathbf{R}^{n} \times \mathbf{R} \times \mathbf{R}^{n}$ a função $u$ ficaria definida como $u: V \rightarrow \mathbf{R}, V \subset \mathbf{R}^{n}$ e $\Omega^{*}$ representará a projeção de $\Omega$ sobre $\mathbf{R}^{n+1}$.

7. A equação de Burgers mostra que é possível determinar o primeiro ponto de interseção das curvas características, depois dele a solução não será de classe $C^{1}$, podendo construir soluções fracas numericamente. Por outro lado construir uma solução fraca para uma EDP implícita de primeira ordem é muito complexo.

8. A equação de Hamiton-Jacobi estabelece a possibilidade do relacionamento das aplicações das EDPs implícitas de primeira ordem. 


\subsection{Trabalhos futuros}

No desenvolvimento do trabalho foi estabelecido que a solução de uma equação diferencial parcial implícita de primeira ordem é equivalente a resolver um sistema de EDOs, além do que no cálculo da solução pode-se considerar que a curva inicial e as curvas características achadas estejam próximas da solução. Se nos garantimos que a função $u$ verifica as condições do teorema de Cauchy podemos estabelecer que o problema consiste em resolver uma equação diferencial sobre uma variedade (Arnold, 1983; Hairer e Wanner, 1993). Um estudo sobre este tema é um trabalho futuro(ver apêndice A ) .

No campo da solução de equações diferenciais sobre variedades é preciso o conhecimento do estudo das equações algébrico diferenciais(EADs), que consideram em seus modelos sistemas de EDOs implícitas, sistemas semi-implícitos ou sistemas de equações diferenciais com restrições em seus variáveis (Brenan e Petzold, 1996; Ascher e Petzold, 1998). A solução numérica de uma EAD permite resolver modelos de EDOs que não possam-se descrever de forma explicita ou que não tenham uma solução por métodos tradicionais(ver apêndice B ).

Dentro das aplicações deste tipo de equações EDOs implícitas e EADs é possível mencionar problemas de sistemas mecânicos, como por exemplo para a simulação da dinâmica de sistemas multi-corpo. Neste caso é possível simular o movimento de um veiculo, ou um teste de segurança de braços robóticos mediante modelos de computação gráfica (Hairer e Wanner, 1993; Ascher e Petzold, 1998). A equivalência existente entre os problemas variacionais e as Equações diferenciais parciais de primeira ordem permite estabelecer problemas de aplicação relacionados com a equação de Hamilton-Jacobi (ver apêndice C ).

Estes três tópicos são estudos que complementam esta dissertação e pretendemos nos aprofundar em um futuro próximo. 

ALLGOWER, E. L.; GEORG, K. Introduction to numerical continuation methods. First ed. Philadelphia: Society for Industrial and Applied Mathematics, 2003.

ARNOLD, V. Geometrical methods in the theory of ordinary diferential equations. First ed. Springer - Verlag, 1983.

ASCHER, U.; PETZOLD, L. Computer methods for ordinay differential-algebraic equations. Siam, 1998.

ATKINSON, KENDALL E. ; HAN, W.; STEWART, D. E. Numerical solutions of ordinary differential equations. John Wiley \& Sons,Inc, 2009.

AWAWDEH, F ; ADAWI, A.; AL-SHARA, S. A numerical method for solving nonlinear integral equations. International Mathematical Forum, , n. 17, p. 805-817, 2009.

Disponível em: <http://www.m-hikari.com/imf-password2009/ 17-20-2009/awawdehIMF17-20-2009.pdf>

BERTIN, M.C. ; PIMENTEL, B. .; POMPEIA, P. Formalismo de hamilton-jacobi à la carathéodory. Revista Brasileira de Ensino de Física, v. 29, n. 3, p. 393-403, 2007.

Disponível em: <http: //www.sbfisica.org.br/rbef/pdf/070306.pdf>

BRAUN, J.; SAMBRIDGE, M. A numerical method for solving partial differential equations on highly irregular evolving grids. Nature, v. 376, p. 655-660, 1995.

Disponível em: <http://wwwrses.anu.edu.au/ malcolm/papers/pdf/bs 95 . pdf>

BRENAN, K.E. ; CAMPELL, S.; PETZOLD, L. Numerical solution of initial-value problems in differential-algebraic equations. Firts ed. Siam, 1996.

BUTCHER, J. Numerical methods for ordinary differential equations. Second ed. John Wiley \& Sons,Inc, 2008. 
CASTELO, A. Aproximações adaptativas de variedades implícitas com aplicações à modelagem implicita e equações algébrico diferenciais. Tese de Doutoramento, 1992.

CASTELO, A.; TAVARES, G. Implicit differential equations with singularities. Relatório Técnico, 2010.

CASTELO, A. ; DE FREITAS, S.; TAVARES, G. PI approximation to manifolds and its application to implicit odes. Lectures in Applied Mathematics, v. 26, p. 99-111, 1990.

CHICONE, C. Ordinary differential equations with applications. Second ed. New York: Springer - Verlag, 2006.

DEBNATH, L. Nonlinear partial differential equations. Third ed. Birkhäuser, 2012.

Disponível em: <http://medcontent.metapress.com/index/ A65RM0 3P $4874243 \mathrm{~N} \cdot \mathrm{pdf}>$

ELSGOLTZ, L. Ecuaciones diferenciales y cálculo variacional. Primera ed. MIR, 1969.

ESPINDOLA, M. L. Equações diferenciais parciais de primeira ordem. SBMAC, v. 72, p. 68, 2014.

Disponível em: <http://www.sbmac.org.br/arquivos/notas/livro_72.pdf>

FARO, R. Apuntes de ecuaciones diferenciales. Badajoz: Universidad de Extremadura, 2013.

FORTUNA, A. D. O. Técnicas computacionais para dinámica dos fluidos conceitos básicos e aplicações. Primeira ed. Editora da Universidade de São Paulo, 2000.

GAMEIRO, M ; LESSARD, J. . J. J. M.; MISCHAIKOW, K. Computational odes, lectures and notes. ICMC, 2013.

GEORGE, P.-L.; FREY, S. Mesh generation application to finite elements. Second ed. John Wiley \& Sons,Inc, 2008.

GOMES, ABEL J.P. ; VICULESCU, I. J. J. . W. B.; GALBRAITH, C. Implicit curves and surfaces :mathematics,data structures and algorithms. Springer - Verlag, 2009.

HAIRER, E. Symmetric projection methods for differential equations on manifolds. Swets and Zeitlinger, v. 40, p. 726-734, 2000.

Disponível em: <https://courses.cs.washington.edu/courses/cse590b/ $02 \mathrm{au} / \mathrm{hairer} 2001 \cdot \mathrm{pdf}>$

HAIRER, E; NØRSETT, S.; WANNER, G. Solving ordinary differential equations $i$, nonstiff problems. First ed. Springer - Verlag, 1993. 
IRENEO, P. Primer curso de ecuaciones en derivadas parciales. Addison Wesley Iberoamericana, 1995.

Disponível em: <http://dialnet.unirioja.es/servlet/libro?codigo= $374307>$

JONH, F. Partial differential equations applied mathematical sciences 1, v. 1. Fourth ed. New York: Springer - Verlag, 1982.

KEVORKIAN, J. Partial differential equations analytical solution techniques. Primera ed. Chapman and Hall, 1990.

LAPIDUS, L.; PINDER, G. Numerical solution of partial differential equations in science and engineering. New York, 2011.

Disponível em: <http://books.google.com/books?hl=en\&lr=\&id= WhyUYUlU7BEC\&Oi=fnd\&pg=PR7\&dq=Numerical+solution+of+partial+ differential+Equations+in+Science+and+engineering\&ots= _1K71MyYYb\&sig=TYAluKwlNkSdNxRJDxjeEscDIQC>

LOGAN, J. D. An introduction to nonlinear partial differential equations. Second ed. John Wiley, 2008.

LÓPEZ, G. Partial differential equations of firts order and their applications to physics. Firts ed. World Scientific Publishing, 1999.

QUARTERONI, A. ; SACCO, R.; SALERI, F. Numerical mathematics. First ed. New York: Springer-Verlag, 2000.

RHEE, H.; AMUNDSON, N. Firts-Order Partial Differential Equations. Prentice-Hall, 1986.

SHAMPINE, L.F. ; GLADWELL, I.; THOMPSON, S. Solving odes with matlab. Cambridge: Cambridge University Press, 2003.

Disponível em: <http://books.google.com/books?hl=en\&lr=\&id= P 4-9gcpqQ_AC\&Oi=fnd\&pg=PR7\&dq=Solving+ODEs+with+MATLAB\&ots= $\mathrm{ZX}-5 \mathrm{kbfS}-7 \& \mathrm{sig}=40 \mathrm{z} 2 \mathrm{k}-\mathrm{KsDPCX}$ CaaUbOjU3gT6jE>

SHAMPINE, L. F. Implementation of implicit formulas for the solution of ode s. SIAM Journal on Scientific and Statistical Computing, v. 1, n. 1, p. 103-118, 1980.

Disponível em: <http://epubs.siam.org/doi/abs/10.1137/0901005>

ZUAZUA, E. Métodos numéricos de resolución de ecuaciones en derivadas parciales. 2009.

Disponível em: <http://www.bcamath.org/documentos_public/archivos/ personal/comites/3_notas-05_065-complete.pdf> 



\section{APÊNDICE}

\section{$A$}

\section{Solução de equações diferenciais sobre variedades}

Dado um sistema de equações diferenciais ordinárias

$$
y^{\prime}=f(y)
$$

com as condições necessárias para garantir sua solução, imaginaremos que a solução está sobre uma variedade ou que é o envelope de uma variedade.

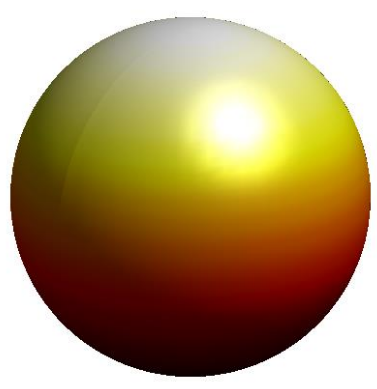

Figura A.1: Esfera

Antes de falar sobre uma Equação Diferencial sobre uma variedade vamos dar algumas definições necessárias. 


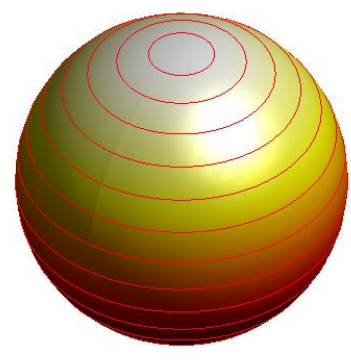

(a) Curvas sobre a esfera

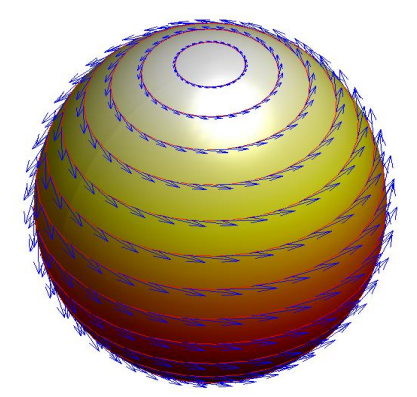

(b) Vetores tangente das curvas sobre uma esfera

Figura A.2: Vetores tangente de curvas sobre uma esfera

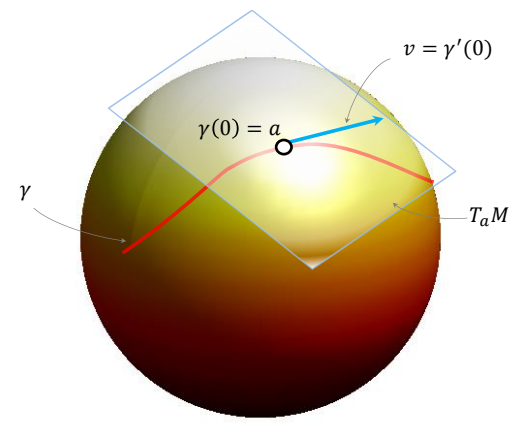

(a) Espaço Tangente

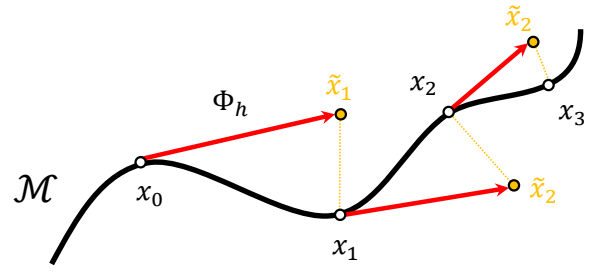

(b) Método previsor corretor

Figura A.3: Espaço Tangente e Método previsor corretor

Definição de Variedade Diferencial: Um conjunto $\mathcal{M} \subset \mathbf{R}^{n}$ é chamado variedade diferenciável de dimensão $n$ e classe $C^{k}$, se cada ponto $x \in \mathcal{M}$ tem uma vizinhança aberta $V \subset \mathbf{R}^{m}$ tal que $V \cap \mathcal{M}$ seja difeomorfo a um aberto $U$ de $R^{n-1} \times R^{+}$. Un difeomorfismo $\phi: U \rightarrow V \cap \mathcal{M}$ é chamado de parametrização da região $V \cap \mathcal{M}$. A fronteira de $\mathcal{M}, \partial \mathcal{M}$ é o conjunto de pontos de $\mathcal{M}$ correspondentes a $\mathbf{R}^{n-1} \times\{0\}$ pelas parametrizações.

Definição de Espaço Tangente: Seja $\mathcal{M} \subset \mathbf{R}^{n}$ uma variedade de $\mathbf{R}^{n}$ e $a \in \mathcal{M}$, o espaço tangente de $\mathcal{M}$ em $a$ é o espaço linear dado por

$T_{a} \mathcal{M}=\left\{v \in R^{n} / \exists \gamma \in C^{1}, \gamma:(-\varepsilon, \varepsilon) \rightarrow R^{n}, \operatorname{com} \gamma(t) \in \mathcal{M}, \forall t \in(-\varepsilon, \varepsilon)\right.$ e $\left.\gamma(0)=a, \gamma^{\prime}(0)=v\right\}$

Se $y(t)$ é uma curva diferenciável com valores em $\mathcal{M} \subset \mathbf{R}^{n}$ então seu derivada $y^{\prime}(t)$ satisfaz $y^{\prime}(t) \in T_{y(t)} \mathcal{M}, \forall t$.

Definição de Equação diferencial sobre uma variedade: Seja $\mathcal{M} \subset \mathbf{R}^{n}$ uma variedade, um campo vetorial sobre $\mathcal{M}$ é um mapeamento $f: \mathcal{M} \rightarrow \mathbf{R}^{n}$ de classe $C^{1}$ tal que $f(y) \in T_{y} \mathcal{M}$, $\forall y \in \mathcal{M}$. Denomina-se equação diferencial sobre a variedade $\mathcal{M}$ a todo campo vetorial $y^{\prime}=f(y)$, 
e a função $y: I \rightarrow \mathcal{M}$ que satisfaz $y^{\prime}(t)=f(y(t)), \forall t \in I$ é chamada curva integral ou solução da equação.

Exemplo: O seguinte sistema descreve as equações de movimento das orbitas sobre uma esfera

$$
\left\{\begin{array}{l}
y_{1}^{\prime}=\left(I_{3}^{-1}-I_{2}^{-1}\right) y_{3} y_{2} \\
y_{2}^{\prime}=\left(I_{1}^{-1}-I_{3}^{-1}\right) y_{1} y_{3} \\
y_{3}^{\prime}=\left(I_{2}^{-1}-I_{1}^{-1}\right) y_{2} y_{1}
\end{array}\right.
$$

se $I_{1}=1.6, I_{2}=1, I_{3}=2 / 3$, e tomando como valor inicial o ponto $y_{0}=(\cos (0.9), 0, \sin (0.9))$, podemos usar algum método numérico descrito por $y_{n+1}=\phi_{h}\left(y_{n}\right)$, como por exemplo o método de Euler, obtendo a solução mostrada nas Figuras A.4(a), A.4(b)

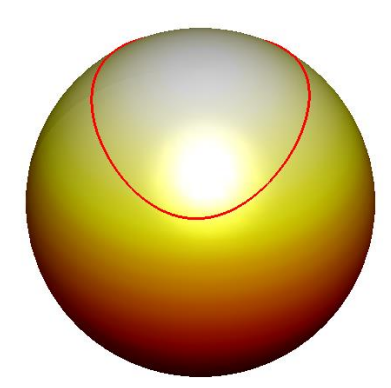

(a) Curva solução sobre a esfera

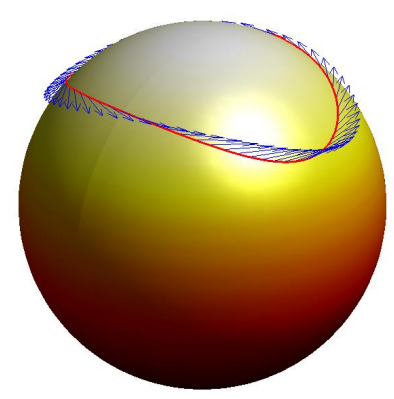

(b) Vetores tangentes da curva solução características

Figura A.4: Solução das Equações do movimento sobre uma esfera

podemos notar graficamente que é preciso não só resolver o sistema se não também fazer a projeção dos pontos sobre a variedade $\mathcal{M}$ como é mostrado nas Figuras A.4(a), A.4(b)

As Equações de movimento sobre uma esfera dadas por (A.2) correspondem a uma equação diferencial sobre a variedade definida por

$$
g\left(y_{1}, y_{2}, y_{3}\right)=y_{1}^{2}+y_{2}^{2}+y_{3}^{2}-R^{2}=0
$$

além disso para o Hamiltoniano dado por

$$
H\left(y_{1}, y_{2}, y_{3}\right)=\frac{1}{2}\left(\frac{y_{1}^{2}}{I_{1}}+\frac{y_{2}^{2}}{I_{2}}+\frac{y_{3}^{2}}{I_{3}}\right)
$$

o sistema Hamiltoniano correspondente a esfera definida por $g=0$ é equivalente ao sistema (A.2)(Hairer, 2000). 
A solução de uma Equação diferencial sobre uma variedade considera a solução de um sistema

$$
y^{\prime}=f(y)
$$

com condição inicial $y(0)=y_{0}$, supondo a existência de um conjunto invariante $\mathcal{M}$ definido pela equação algébrica $0=h(y)$ tal que se $h\left(y_{0}\right)=0$ então $h(y(t))=0, \forall t$. Nos diversos modelos encontram-se relacionado o Hamiltoniano que através das seu sistema canônico descreve o movimento de pontos sobre uma variedade. 


\section{Métodos numéricos para equações algébrico diferenciais}

Um problema de valor inicial refere-se a um sistema de EDOs da forma

$$
x^{\prime}=f(t, x)
$$

Nem sempre é possível descrever uma EDO em forma explicita assim como a EDO (B.1). Em alguns casos ela pode ter a forma implícita

$$
F\left(t, x, x^{\prime}\right)=0
$$

Uma extensão de uma EDO explicita são as EDOs com restrições que são EDOs que dependem de uma equação algébrica como por exemplo a seguinte equação

$$
\left\{\begin{array}{l}
x^{\prime}=f(t, x, z) \\
0=g(t, x, z)
\end{array}\right.
$$

então o sistema dado pela equação B.3 é denominado sistema semi-explicito.

Descrever uma EDO em forma implícita ou semi-implícita faz questionar a existência e unicidade destas equações por exemplo numa equação semi-implícita como B.3 pode não ter solução única.

Para uma EDP implícita de primeira ordem se associamos seu sistema característico a um modelo de equações algébrico diferencias semi-implícito 


$$
\begin{gathered}
\begin{cases}\frac{d X}{d s}=F_{p} & X(0)=X(t) \\
\frac{d Y}{d s}=F_{q} & Y(0)=Y(t) \\
\frac{d U}{d s}=p F_{p}+q F_{q} & U(0)=U(t) \\
\frac{d P}{d s}=-F_{x}-p F_{u} & P(0)=P(t) \\
\frac{d Q}{d s}=-F_{y}-q F_{u} & Q(0)=Q(t)\end{cases} \\
\begin{cases}0=U^{\prime}(t)-P(t) X^{\prime}(t)-Q(t) Y^{\prime}(t) \\
0=F(X(t), Y(t), U(t), P(t), Q(t))\end{cases}
\end{gathered}
$$

temos que uma EDO converte-se em uma EAD, mas se é resolvida como uma EDO, pode falhar na hora de satisfazer as restrições algébricas.

Um abordagem na solução do sistema característico considerando (B.4) e (B.5) como um sistema semi-implícito poderia permitir achar sua solução, pois assim poderíamos achar os vetores normais diretamente resolvendo primeiro o sistema (B.5) e substituindo logo em (B.4), pudendo realizar uma combinação de diversos métodos para EADs.

Existem alguns modelos de problemas clássicos da Mecânica cuja formulação corresponde a um sistema semi-implícito, podemos mencionar as equações de movimento descritas por

$$
\begin{aligned}
q^{\prime} & =v \\
M(t, q) v^{\prime} & =f(t, q, v)-G^{T}(t, q) \lambda, . \\
0 & =g(t, q)
\end{aligned}
$$

onde $G=\frac{\partial g}{\partial q}, M$ é uma matriz da massa positiva definida generalizada, $f$ as forças aplicadas e $v$ as velocidades generalizadas, o sistema depende das coordenadas de posição generalizada $q$. 
APÊNDICE

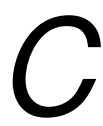

\section{Equivalência de Equações Diferenciais Parciais de Primeira Ordem e Problemas Variacionais}

Existem diferentes problemas de otimização e controle de natureza variacional, daqui a importância de utilizar a equivalência existente com as EDP de primeira ordem com a finalidade de resolver estes problemas.

Por exemplo o sistema de controle especificado por $n$ variáveis de estado $x_{i}(t)$ e $m$ variáveis de controle $u_{j}(t)$, se $x=\left(x_{1}, \ldots, x_{n}\right)$ e $u=\left(u_{1}, \ldots, u_{m}\right)$ o sistema dado por

$$
\dot{x}=f(x ; u)
$$

onde $f=\left(f_{1}, \ldots, f_{n}\right)$ sendo $f_{i}$ e suas derivadas primeiras continuas, se o problema tem especificados valores de iniciais $x_{i}(0)=\xi_{i}$ e queremos achar o controle permissível que minimiza

$$
\int_{0}^{\theta} f_{0}(x ; u) d t+h(x(\theta))
$$

para uma duração de controle $\theta$. A expressão $f_{0}(x ; u)$ é o custo por unidade de tempo no estado $x(t)$ e o controle $u(t)$, e $h(x(\theta))$ é o custo associado com a finalização no estado $x(\theta)$. Denotando $\theta$ por $\xi_{n+1}$ e $x_{n+1}=\theta-t$, assim o minimo valor da integral é uma função dada como

$$
g\left(\xi_{1}, \ldots, \xi_{n+1}\right)=\min _{u}\left[\int_{0}^{\xi_{n+1}} f_{0}(x ; u) d t+h\left(x\left(\xi_{n+1}\right)\right)\right]
$$


a função $g$ satisfaz

$$
\min _{u}[H(\xi, p, u)]-p_{n+1}=0
$$

onde

$$
\xi=\left(\xi_{1}, \ldots, \xi_{n}\right), p_{i}=\frac{\partial g}{\partial \xi_{i}}
$$

e

$$
H=f_{0}(\xi, u)+\sum_{j=i}^{n} f_{j}(\xi, u) p_{j}
$$

O principio do minimo ou máximo de Pontryagin's estabelece que condições são necessárias para que este problema seja um problema de controle ótimo em que $H$ tenha um mínimo absoluto com respeito a $u$ ((Rhee e Amundson, 1986))

Em outro exemplo temos o problema de minimizar o funcional

$$
J[x]=\int_{\alpha}^{\beta} L\left(t, x^{i}, \dot{x}^{i}\right) d t
$$

sujeito as restrições $x^{i}(\alpha)=a^{i}, x^{i}(\beta)=b^{i}$, onde $a, b$ são pontos dados. A solução deste problema é uma curva $x(t)$ chamada geodésica entre os pontos $a$ e $b$ o valor de $J$ é a distância geodésica, se consideramos $a$ fixo e deixamos $b$ variar podemos definir a distância geodésica como

$$
S(b)=S\left(b^{1}, \ldots, b^{n}, \beta\right)
$$

que é uma função que depende do ponto final partindo do ponto $a$, o problema satisfaz a equação de Hamilton-Jacobi

$$
S_{\beta}+H\left(\beta, b^{i}, S_{b^{i}}\right)=0
$$

Em ambos casos notamos que existe uma conexão entre o cálculo variacional e a solução de Equações Diferenciais Parciais implícitas de Primeira Ordem a traves da equação de HamiltonJacobi(Rhee e Amundson, 1986), a teoria desenvolvida no trabalho e os métodos numéricos poderiam ser avaliados para sua utilização e achar uma solução numérica para estes problemas. 